Microbial chain elongation based on methanol

\author{
Wei-Shan Chen
}




\section{Thesis committee}

\section{Promotors}

Prof. Dr C.J.N. Buisman

Professor of Biological Recovery and Re-use Technology

Wageningen University \& Research

Prof. Dr C. Kroeze

Professor of Water Systems and Global Change

Wageningen University \& Research

\section{Co-promotor}

Dr D.P.B.T.B. Strik

Assistant professor, Sub-department of Environmental Technology

Wageningen University \& Research

\section{Other members}

Prof. Dr A.J.M. Stams, Wageningen University \& Research

Prof. Dr L. Diels, University of Antwerp, Belgium

Prof. Dr C.A. Ramirez Ramirez, Delft University of Technology

Dr T.I.M. Grootscholten, Royal Cosun, Roosendaal, The Netherlands

This research was conducted under the auspices of the Graduate School for Socio-Economic and Natural Sciences of the Environment (SENSE) 


\title{
Microbial chain elongation based on methanol
}

\author{
Wei-Shan Chen
}

Thesis

submitted in fulfilment of the requirements for the degree of doctor

at Wageningen University

by the authority of the Rector Magnificus,

Prof. Dr A.P.J. Mol,

in the presence of the

Thesis Committee appointed by the Academic Board

to be defended in public

on Wednesday 21 June 2017

at 4 p.m. in the Aula. 


\section{Wei-Shan Chen}

Microbial chain elongation based on methanol

201 pages

PhD thesis, Wageningen University, Wageningen, the Netherlands (2017)

With references, with summary in English

ISBN: 978-94-6343-198-9

DOI: $10.18174 / 413896$ 
To my beloved family, especially my grandpa and parents 



\section{Table of contents}

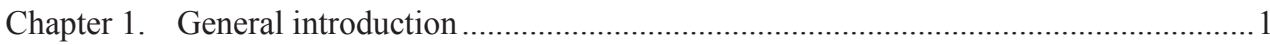

Chapter 2. Life Cycle Assessment (LCA) of microbial chain elongation ...........................21

Chapter 3. Methanol chain elongation for butyrate and caproate production .......................55

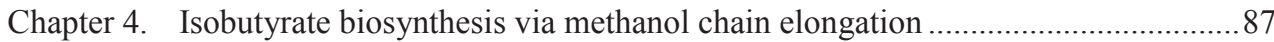

Chapter 5. Concurrent use of methanol and ethanol for chain-elongating short chain fatty

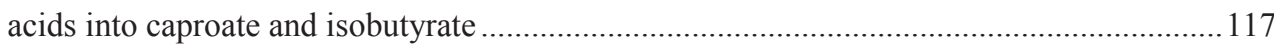

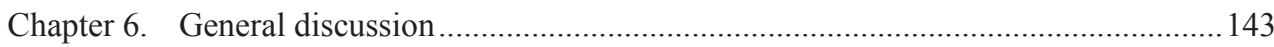

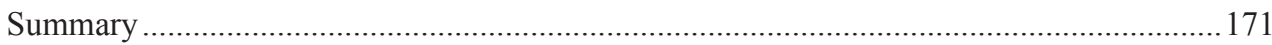

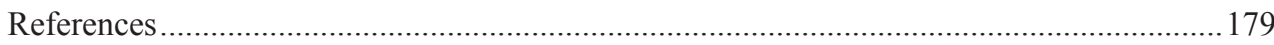

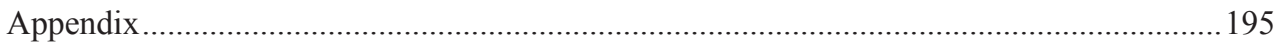



Chapter 1. General introduction 


\subsection{Demand for a sustainable production chain}

The growing world population, economic development, and increasing human welfare go hand in hand. Large quantities of energy and materials are needed to sustain these growing economies. Currently, the energy and materials used in the global economy are mainly produced from fossil resources with about $80 \%$ of the global energy demand currently met through the use of fossil fuels ${ }^{1}$.

This dependency on fossil fuels hinders our society from being economically and environmentally sustainable for several reasons. Firstly, fossil fuels contain large amounts of carbon that have been sequestrated underground during the past few million years. Burning fossil fuels releases this carbon back into the atmosphere within a relatively short period of a few centuries, in the form of carbon dioxide $\left(\mathrm{CO}_{2}\right)$; one of the major greenhouse gases (GHG) contributing to global warming. $\mathrm{CO}_{2}$ emissions from fossil fuel consumption currently accounts for around two-thirds of the total global GHG emissions ${ }^{2}$. Fossil fuels are considered a non-renewable resource that cannot continue to sustain our future global economic system in a sustainable way ${ }^{3}$. Secondly, the uneven distribution of fossil reserves across the globe can be a cause of socio-economic insecurity, as illustrated by the oil crisis during the 1970s. Developed and industrialised regions without oil reserves are especially vulnerable in this respect, as oil is likely to be the first fossil resource to be exhausted ${ }^{4}$. Finally, the transportation of fossil resources requires a substantial amount of energy, which could be avoided or reduced by employing locally available feedstocks for energy and material production. Replacing fossil resources with alternatives would therefore help us to sustain our society and its foreseeable growth in the long run. The preferred alternative energy and materials supplied to our society are from resources that are more renewable, environmentally sustainable and locally available. 
To ensure a more sustainable future for our society, a circular economy is preferred. However, the current economy is far from circular. The Industrial Revolution marks the start of the fossil-based production chains. Since then, the global economy has been developing into linear systems. These linear economic systems start with resource extraction from nature, go through energy and goods production and consumption, and eventually end with waste generation and treatment. These linear economic systems produce large quantities of waste. It has been estimated that 1.3 billion tonnes of municipal solid waste (MSW) is generated every year globally, and this does not yet include waste produced in rural areas ${ }^{5}$. We may define the entire earth as the system boundary for the expansion of our economic activities. Many resources within this global system must be considered finite, and our current practices based on the aforementioned linear approach cannot be considered economically and environmentally sustainable. Transition to a circular economy requires that, after consumption, the resources and waste are converted into reusable energy or materials to be re-supplied to the global economy.

\subsection{Bio-based economy as a solution: state-of-art}

A bio-based economy may help in the transition of the current fossil-based, linear economy to a sustainable and circular economy. In a bio-based economy, biomass is used as a feedstock for producing energy, materials, chemicals and transportation fuels, in addition to food and feed. Biomass feedstocks can regrow relatively quickly, making them more renewable than fossil-based feedstocks. During the growth of biomass feedstocks, nutrients and carbon are captured and sequestrated into the biomass that will become part of new products again. This fulfils the concept of a circular economy. A transition towards a more circular economy has slowly started and is continuously progressing as a result of public awareness of sustainability issues and environmental pollution caused by the fossil-based, linear economic system. 


\section{Chapter 1}

The first generation biofuels are an example of the advancement in the bio-based economy. Bioethanol from sugar crops, e.g. corn and sugarcane, and biodiesels from oil plants, e.g. palm and coconut, are examples of first generation biofuels that are implemented at commercial scales and used extensively as drop-in fuels in the transportation sector. The global annual bioethanol and biodiesel production was estimated at around 39 and 51 billion litres respectively, and these numbers are expected to grow over time ${ }^{6,7}$. Nevertheless, in comparison with the fossil oil production of 3,900 billion litres in $2007^{6}$, bioethanol and biodiesel have only a small share in the global production chain.

First generation biofuels, however, have some important drawbacks leading to increasing concerns. Examples of drawbacks include the large water and nutrient requirements, competition with arable land, increased GHG emissions due to direct and indirect land use change, and the geographical limitations for growing these crops. The water footprints of first generation biofuels are much larger than fossil-based fuels, when used for energy generation. Expanding the production of first generation biofuels may cause competition with other sectors for fresh water resources ${ }^{8}$. The use of sugar and oil crops require fertile, arable land, which may compete with food production. Also, the indirect land use change for producing biofuels/biochemicals leads to more GHG emissions ${ }^{9}$ and induces other environmental impacts besides global warming ${ }^{10}$. Although debates about the land competition between food and fuel production are on-going ${ }^{11}$, it is inevitable that more arable land is required for food production due to the ever increasing world population and the growing calorie uptake due to improvements in quality of life. The use of arable land for fuel and chemical production should be minimised. Moreover, due to the fresh water, arable land and climate requirements for crops, first generation biofuels have a geographical limitation, which is also an issue for fossil resources. In 2007, USA and Brazil together held $90 \%$ of the global bioethanol production ${ }^{6}$, while five countries (Malaysia, Indonesia, Argentina, USA and 
Brazil) owned $80 \%$ of the global biodiesel production potential ${ }^{7}$. Given the context above, fuel and chemical production has to come from other feedstocks that are neither food cropbased or fossil-based ${ }^{12}$.

\subsection{Sustainable feedstocks for a sustainable bio-based economy}

To promote a bio-based and more sustainable economy, alternative feedstocks are needed as starting materials to produce fuels and chemicals. There are five biomass resources identified as "sustainable feedstocks" for fuel and chemical production, mainly due to their relatively low life-cycle GHG emissions and lack of competition with food production ${ }^{13}$. Based on Tilman et al. (2009), these are (1) perennial plants grown on degraded land abandoned from agricultural use, (2) crop residues, (3) sustainably harvested wood and forest residues, (4) double crops/mixed crop systems and (5) municipal and industrial organic waste $^{13}$. Biofuels produced from these feedstocks are classified as second generation biofuels. In the following sections, I refer to these feedstocks also as second generation biomass. Second generation biomass can also be categorised, based on the composition, into three groups, i.e. the lignocellulosic biomass (1,2 and 3 above), the triglycerides ( 4 and 5 above) and the mixed organic waste $(5 \text { above })^{14}$. Sugar/starch crops in the double crops/mixed crop systems are not included in this categorisation as they may be used for food supply. Second generation biomass is more geographically-unbound compared with fossil resources and first generation biomass.

Most of the second generation biomass is generally considered "waste", requiring additional capital and energy investments to dispose of. The use of second generation biomass to produce energy, fuel and materials is beneficial for managing the energy and material supply, in addition to waste treatment, simultaneously. This is especially the case for chemicals and materials, since fuels and energy for transportation can also be provided by other renewable sources such as solar energy. In contrast, for chemicals and materials, carbon 


\section{Chapter 1}

atoms as basic building blocks are inevitable. Thus, biomass is an essential source of carbon. Promoting the use of second generation biomass for chemical and material production can, therefore, be important in a circular economy.

\subsection{Conversion technologies and potential end-product for second generation biomass}

The conversion of second generation biomass, especially the lignocellulosic feedstock and mixed organic waste, into value-added chemicals and materials is challenging because of their composition. Lignocellulosic feedstock contains carbohydrate polymers (cellulose and hemicellulose) and aromatic polymers (lignin) that are rigidly bound together, which makes them difficult to decompose ${ }^{15}$. To effectively decompose lignocellulose, either an enzymatic hydrolysis or a thermochemical conversion process, e.g. gasification, is usually employed. A mixed sugar stream is derived via the enzymatic hydrolysis of lignocellulosic biomass, which is then fermented into ethanol or other fermentative products. Synthesis gas (syngas; a mixture of carbon monoxide, $\mathrm{CO}_{2}$ and hydrogen $\left(\mathrm{H}_{2}\right)$ ) is the main product of the gasification of lignocellulosic biomass ${ }^{16,17}$. Syngas can be converted into methanol via a chemical reaction $^{18}$, ethanol or fatty acids via a (bio)chemical conversion ${ }^{19}$ or liquid hydrocarbons via the Fischer-Tropsch process.

Mixed organic waste varies in composition. It is normally highly heterogeneous and with a high water content. This dynamic and heterogeneous composition makes it challenging to convert mixed organic waste into a homogenous end-product, while the high water content makes energy recovery via thermochemical treatments less energy-efficient. Current practices for valorising mixed organic waste are incineration for energy recovery, composting for generating soil amendment and anaerobic digestion for producing methane as an energy carrier. Incineration only recovers energy from the mixed organic waste but no other valueadded carbon materials. Both composting and anaerobic digestion can recover useful 
materials from organic waste; however, the low economic value of their end-products means that it is difficult for these methods to be economically viable without subsidies ${ }^{20,21}$. An innovative valorisation strategy for mixed organic waste that is more cost-effective and recovers carbon materials in the form of value-added materials is therefore demanded.

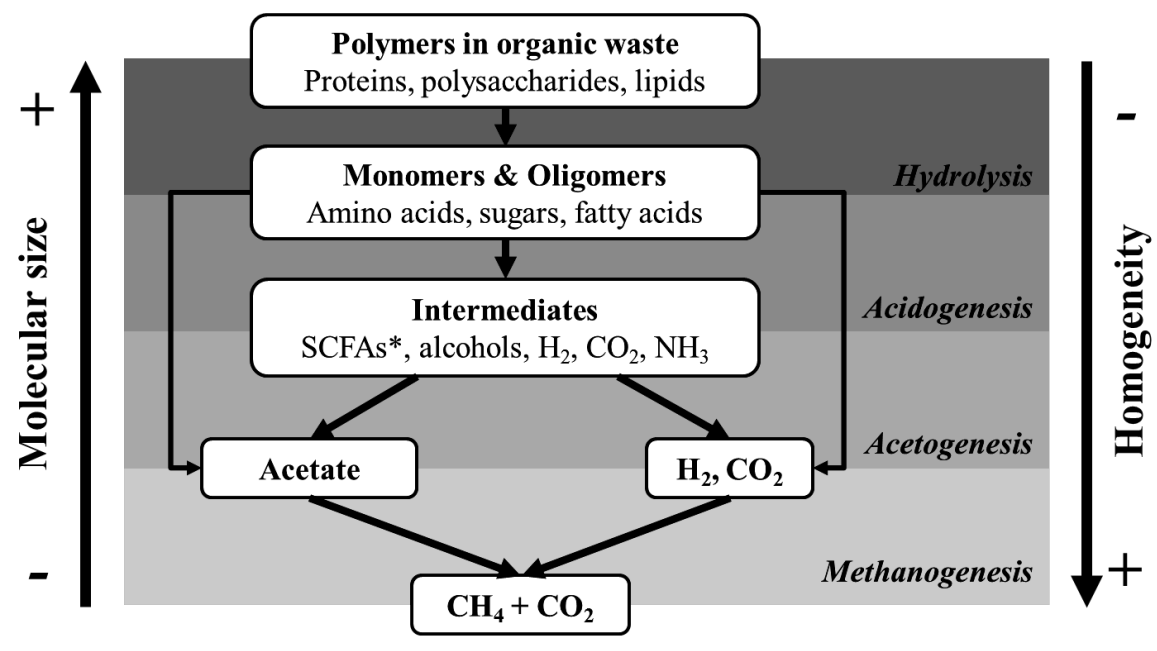

Figure 1.1. The stepwise illustration of the methanogenic anaerobic digestion of the complex mixed organic waste, adapted from Steinbusch et al. $(2010)^{22}$ and Angenent et al. $(2004)^{23}$. *Short Chain Fatty Acids (SCFAs).

Open mixed culture fermentation is a promising alternative for valorising the mixed organic waste in terms of material, feed and chemical production. Open mixed culture refers to a mixed culture microbial community that is open to the environment outside of its habitat, i.e. accessible by microbes outside of its habitat. In fact, the methanogenic anaerobic digestion of complex mixed organic waste can also be considered as an open mixed culture fermentation. The microbes in mixed organic waste continually enter, along with the waste, the bioreactor where a mixed culture already exists. There are four main steps in the methanogenic anaerobic digestion (see Figure 1.1). First is hydrolysis, which breaks down the large, insoluble organic polymers into smaller, soluble organic polymers with enzymes 


\section{Chapter 1}

via a chemical reaction with water. During the second step, acidogenesis, a mixture of fermentative bacteria converts dissolved polymers into monomers e.g. Short Chain Fatty Acids (SCFAs), alcohols, $\mathrm{H}_{2}$ and $\mathrm{CO}_{2}$. In the third step, acetogenesis, these monomers are further converted into acetate, $\mathrm{H}_{2}$ and $\mathrm{CO}_{2}$. In the final step (methanogenesis), the methanogens convert acetate, $\mathrm{H}_{2}$ and $\mathrm{CO}_{2}$ into methane $\left(\mathrm{CH}_{4}\right)$ and $\mathrm{CO}_{2}$, i.e. biogas. Along with this methanogenic degradation process, the molecular size of the organic matters in the waste decreases, whilst the homogeneity increases (Figure 1.1). The ability to homogenise the complex mixed organic waste into a relatively simple mixture of intermediates, e.g. SCFAs, $\mathrm{H}_{2}$ and $\mathrm{CO}_{2}$, is one of the most important advantages of open mixed culture fermentation. In current practices, these intermediates are converted into $\mathrm{CH}_{4}$ and $\mathrm{CO}_{2}$, socalled biogas. However, $\mathrm{CH}_{4}$ has a relatively low economic value, limited applications (usually for energy generation) and a lower carbon recovery efficiency. Novel bioprocesses have been developed in recent years to recover carbon materials from mixed organic waste in a more cost-effective manner. This includes the production of liquid fuel (ethanol) ${ }^{24,25}$, bulk chemicals (fatty acids) ${ }^{26,27}$ and materials (bioplastics) ${ }^{28}$. Table 1.1 lists the carbon recovery efficiencies of several bioprocesses which convert organic matter into energy carrier $\left(\mathrm{CH}_{4}\right)$, chemicals and materials, using glucose as a model substrate. Based on the carbon recovery efficiencies of glucose, producing fatty acids retains the most carbon materials in the endproduct and also emits less $\mathrm{CO}_{2}$, when compared with the other bioprocesses listed. Moreover, medium chain fatty acids (MCFAs; saturated monocarboxylates with a carbon chain containing 6-12 carbon atoms), e.g. caproate and caprylate, have higher volumetric energy densities than ethanol or $\mathrm{CH}_{4}$. The longer carbon chains of MCFAs make them more hydrophobic and potentially easier to be separated from the aqueous phase, than SCFA. All these properties make MCFAs promising end-products which result from mixed organic waste. 
Table 1.1. Carbon recovery efficiencies of different biochemical conversions of organic matter, taking glucose as a model substrate. The efficiencies are theoretical values, with calculations based on the reaction stoichiometry without considering the energy requirement for the bacterial metabolism.

Equation Biochemical conversion process $\uparrow$

1 Methanogenic anarobic digestion of glucose

$\mathrm{C}_{6} \mathrm{H}_{12} \mathrm{O}_{6} \rightarrow 3 \mathrm{CH}_{4}+3 \mathrm{CO}_{2}$

Anaerobic fermentation of glucose to caproate

$$
\mathrm{C}_{6} \mathrm{H}_{12} \mathrm{O}_{6} \rightarrow 0.75 \mathrm{C}_{6} \mathrm{H}_{12} \mathrm{O}_{2}+1.5 \mathrm{CO}_{2}+1.5 \mathrm{H}_{2} \mathrm{O}
$$

3

Anaerobic fermentation of glucose to butyrate

$$
\mathrm{C}_{6} \mathrm{H}_{12} \mathrm{O}_{6} \rightarrow 1.2 \mathrm{C}_{4} \mathrm{H}_{8} \mathrm{O}_{2}+1.2 \mathrm{CO}_{2}+1.2 \mathrm{H}_{2} \mathrm{O}
$$

4

$$
\text { Anaerobic fermentation of glucose to acetate }
$$$$
\mathrm{C}_{6} \mathrm{H}_{12} \mathrm{O}_{6} \rightarrow 3 \mathrm{C}_{2} \mathrm{H}_{4} \mathrm{O}_{2}
$$

5

$$
\begin{aligned}
& \text { Anaerobic fermentation of glucose to ethanol } \\
& \mathrm{C}_{6} \mathrm{H}_{12} \mathrm{O}_{6} \rightarrow 2 \mathrm{C}_{2} \mathrm{H}_{6} \mathrm{O}+2 \mathrm{CO}_{2}
\end{aligned}
$$

6

Aerobic conversion of glucose to polyhydroxybutyrate (PHB) monomers ${ }^{29}$

$$
\mathrm{C}_{6} \mathrm{H}_{12} \mathrm{O}_{6}+1.5 \mathrm{O}_{2} \rightarrow \mathrm{C}_{4} \mathrm{H}_{6} \mathrm{O}_{2}+2 \mathrm{CO}_{2}+3 \mathrm{H}_{2} \mathrm{O}
$$

Maximal carbon recovery in the end-product (\%)

$50 \%$

$75 \%$

$80 \%$

$100 \%$

$66 \%$

$66 \%$ 


\subsection{Microbial chain elongation with a mixed culture: State-of- art}

To produce MCFAs from organic waste, two open mixed culture fermentation steps followed by a downstream process are applied. The first step is the hydrolysis and acidification (including acidogenesis and acetogenesis; Figure 1.1). Second is the microbial chain elongation. In microbial chain elongation, SCFAs, $\mathrm{CO}_{2}$ and/or $\mathrm{H}_{2}$ produced via hydrolysis and acidification of organic waste, are coupled with ethanol (as an electron donor) to produce MCFAs such as caproic acid and caprylic acid ${ }^{30}$. It was recently (2010) discovered that microbial chain elongation could be performed with undefined open mixed cultures $^{31,32}$. Ding et al. (2010) showed that caproate was produced with undefined open mixed cultures as a side product during hydrogen production from glucose. Similar findings were reported by Steinbusch et al. (2011), who could enhance the caproate production to a higher concentration $(9.6 \mathrm{~g} / \mathrm{L})$. Instead of using sugar, acetate with ethanol was used as the substrate and electron donor. Steinbusch et al. (2011) also found that a longer chain fatty acid, caprylate (C8), could be produced. These recent findings enable the production of higher value chemicals from low grade biomass and organic waste. Its novelty and importance were addressed in several recent reviews ${ }^{26,33,34}$.

Following the first demonstration of microbial chain elongation with an open mixed culture $^{32}$, several studies continued to improve the production rate $^{35,36}$, the utilisation of a real waste stream as the feedstock ${ }^{37-40}$ and the downstream processing for MCFAs ${ }^{37-39}$. Grootscholten et al. (2013) achieved a 3000-fold increase in the caproate production rate by using an upflow anaerobic filter ${ }^{36}$ and reducing the hydraulic retention time $(\mathrm{HRT})^{35}$. Additionally, their work showed that both product yield and specificity are increased to a level of industrial relevance ${ }^{35}$. Grootscholten et al. (2013) also demonstrated the use of organic fraction of municipal solid waste (OFMSW) as the feedstock for MCFA production ${ }^{38,}$ 
39. As such, a spin-off company, ChainCraft B.V. (Amsterdam), is up-scaling and commercialising microbial chain elongation, with supermarket food waste as the raw material and caproic acid as the first commercial product ${ }^{33}$. All these progresses make microbial chain elongation a promising and serious biorefinery process that can become a relevant platform technology for society.

\subsection{Searching for alternative electron donors and novel products for microbial chain elongation}

To make microbial chain elongation more cost-effective, practically-applicable and geographically-unbound, the search for alternative electron donors to replace or reduce ethanol use was recommended ${ }^{30}$. A potential drawback of microbial chain elongation is the addition of expensive chemicals, e.g. ethanol, yeast extract and micronutrients, as stated by Grootscholten $(2015)^{30}$. Ethanol is a more expensive substrate in microbial chain elongation, in comparison with the SCFAs from organic waste. In contrast, micronutrients and amino acids provided by yeast extract can be present in the food waste employed as the feedstock for microbial chain elongation, which may not require extra doses. Currently, most commercially available ethanol is derived from the fermentation of crop-based sugars, i.e. first generation biomass. Since 2.2 moles of ethanol are needed to synthesise 1 mole caproate from 1 mole acetate ${ }^{26,41}$, ethanol dosing in microbial chain elongation can become massive, and consequently induce a series of environmental impacts and high costs, if produced from sugar crops. Alternative feedstocks that are cheaper, widely available and produced in a more renewable way are needed as electron donors in the future development of microbial chain elongation.

In recent years, several alternative electron donors from different feedstocks were investigated to replace or reduce the use of crop-based ethanol in microbial chain elongation. These efforts are summarised in Figure 1.2. There are two strategies for developing 


\section{Chapter 1}

alternative feedstocks for electron donors. One is to employ a different substrate as the electron donor, which usually occurs via different microbial pathways than the microbial chain elongation using ethanol (hereinafter referred to as ethanol chain elongation). The other is to produce ethanol from second generation biomass. For the first strategy, alternative substrates investigated include $\mathrm{H}_{2}{ }^{32}$, electricity ${ }^{42}$, lactate ${ }^{43,44}$, syngas ${ }^{45,46}$ and methanol ${ }^{47}$. All these electron donors have been tested in microbial chain elongation with an open mixed culture except for methanol, which used a pure culture. For the second strategy, ethanol from syngas fermentation (pure culture) and from biohydrogenation of acetate (open mixed culture) were used for microbial chain elongation of $\mathrm{SCFAs}^{40,48}$.

Among all the alternative substrates studied, methanol represents a promising substrate, mainly because methanol can be, and is, produced from organic waste that is widely available geographically $^{49,50}$. Lignocellulosic waste, industrial off-gas ${ }^{51}$, glycerol ${ }^{52}$ and $\mathrm{CO}_{2}$ with renewably produced $\mathrm{H}_{2}$, are all examples of renewable feedstocks used for methanol production. Several initiatives have been taken to implement and upscale methanol production from renewable feedstocks, especially in Europe ${ }^{50}$. These initiatives and the renewable feedstocks used for methanol production include BioMCN (The Netherlands; now acquired by OCI N.V., The Netherlands) using glycerol or wood chips, BioDME (Sweden) using black liquor (a side stream from paper industry), Värmlandsme tanol (Sweden) using forest residue and Carbon Recycling International (Vulcanol; Iceland) using factory off-gas (mainly $\mathrm{CO}_{2}$ ) and $\mathrm{H}_{2}$ (via electrolysis of water with renewable electricity). 


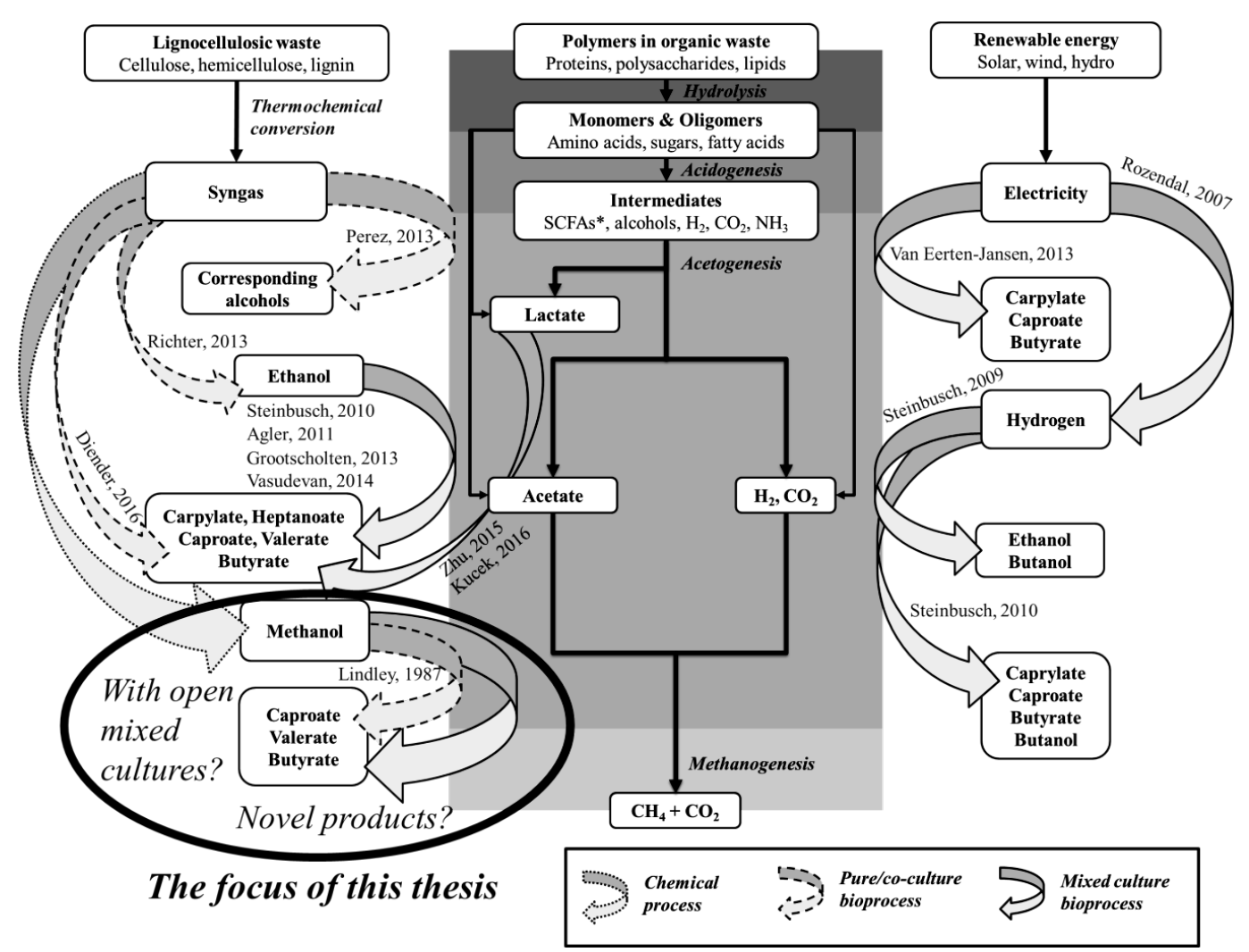

Figure 1.2. The overview of reported processes related to the use of microbial chain elongation for valorising organic waste. Linear arrows refer to biochemical conversion processes within methanogenic anaerobic digestion of mixed organic waste. The thicker arrows refer to different processes that can be integrated with microbial chain elongation. The black circle highlights the research focus of this thesis.

Methanol seems both financial and environmentally more attractive than ethanol, especially when produced from lignocellulosic waste via thermochemical conversion process. According to the National Renewable Energy Laboratory (NREL) of the U.S. Department of Energy (DOE), when processing the same amount of lignocellulosic feedstock, producing methanol has both a higher yield and a higher energy production, combined with a lower capital investment and less water consumption, than ethanol production. As a result, 


\section{Chapter 1}

lignocellulose-based methanol is cheaper than ethanol when evaluated based on price per mole carbon and energy content ${ }^{53}$. Table 1.2 summarises the prices of the main chemicals in this thesis work from different sources including literature, Chinese market valuations and suggestions from industrial partners. Lignocellulosic methanol is cheaper than lignocellulosic and fossil-based ethanol, though still more expensive than crop-based bioethanol. In addition to financial considerations, methanol production from lignocellulose via a thermochemical process could be more environmentally sustainable than ethanol from the same feedstock ${ }^{54}$. All these advantages make methanol an interesting alternative electron donor for microbial chain elongation.

The main obstacle for integrating methanol into the current microbial chain elongation process using mixed organic waste, is the use of methanol in microbial chain elongation using an open mixed culture. Until now, methanol chain elongation to MCFAs via microbial conversion has only been shown in pure culture conditions ${ }^{47,55}$. In an open mixed culture condition, methanol may be metabolised by other competing microbial process, e.g. methylotrophic methanogenesis. Whether microbial chain elongation of methanol and SCFAs into MCFAs or other value-added products can be steered to outcompete other competing microbial processes in an open mixed culture environment remains unknown. An open mixed culture process is necessary when using mixed organic waste as the feedstock, considering the heterogeneous composition and the complex microbiome within mixed organic waste. This research examines the use of methanol chain elongation under an open mixed culture condition (hereinafter referred as methanol chain elongation), investigates the key parameters for steering methanol chain elongation in an open mixed culture and explores novel products from methanol chain elongation. Exploring novel products of microbial chain elongation is important for broadening the application of microbial chain elongation, which also promotes the development of bio-based economy ${ }^{56}$. Caproic acid is currently the only (almost) 
commercialised product of microbial chain elongation. It is an emerging platform chemical with a limited market size, though it has a high economic value. Considering the biodiversity of the open mixed culture and possible interactions among microbes within it, steering the microbiome towards novel products may be possible. Isomerised fatty acids, e.g. isobutyrate, are an example demonstrated in this research (Chapters 4 and 5), which has a relatively high economic value (Table 1.2), a larger market potential ${ }^{57}$ and is currently produced from fossilbased feedstocks ${ }^{57}$. 
Chapter 1

Table 1. 2. Price overview of the substrates and (potential) products mentioned in this study.

\begin{tabular}{|c|c|c|}
\hline Chemical & Price $(€ / \mathrm{L})$ & Price $(€ / \text { mole-Carbon })^{*}$ \\
\hline \multirow[t]{2}{*}{ Methanol } & 0.3 (lignocellulose) $^{53}$ & 0.012 \\
\hline & $0.5^{\dagger}$ & 0.018 \\
\hline \multirow[t]{4}{*}{ Ethanol } & $0.2-0.4$ (sugar $^{58}$ & 0.006 \\
\hline & 0.5 (lignocellulose) $^{53}$ & 0.015 \\
\hline & $0.7{\text { (fossil })^{58}}^{58}$ & 0.020 \\
\hline & $0.45^{\#}$ & 0.013 \\
\hline Acetate & $0.6^{59}$ & 0.017 \\
\hline \multirow[t]{2}{*}{ Propionate } & $1.4^{\dagger}$ & 0.035 \\
\hline & $0.9^{\#}$ & 0.022 \\
\hline Butyrate & $1.1-1.4^{\dagger}$ & 0.025 \\
\hline Valerate & $2.9-3.2^{\dagger}$ & 0.064 \\
\hline \multirow[t]{3}{*}{ Caproate } & $2.0^{59}$ & 0.042 \\
\hline & $7.6^{\dagger}$ & 0.158 \\
\hline & $2.5-3.0^{\#}$ & $0.052-0.062$ \\
\hline Isobutyrate & $1.8^{\dagger}$ & 0.041 \\
\hline Isovalerate (3-methylbutanoate) & $4.8^{\dagger}$ & 0.105 \\
\hline
\end{tabular}




\subsection{Research objective, questions and the outline of the thesis}

The objective of this thesis is to investigate the use of methanol as an electron donor in microbial chain elongation for producing MCFAs or other novel products. The motivations to use methanol instead of ethanol include the potentially lower cost, the potentially lower environmental impact and the geographically-unbound availability of methanol. The motivation for investigating possible novel products is to promote the application of microbial chain elongation, considering the currently limited market potential of caproic acid. Table 1.3 summarised the individual research questions, the substrate and target products of each research chapter.

In Chapter 2, we performed a Life Cycle Assessment (LCA) to assess the environmental impacts of the current microbial chain elongation process, which converts organic waste and ethanol into caproic acid. Performing an LCA in an early stage of technology development is beneficial for improving the process design, and for identifying hotspots where more research or improvements are needed ${ }^{60}$. Through an LCA, the necessity to replace ethanol from the environmental point of view can be further explored. In Chapter 3, the use of methanol as the sole electron donor for chain-elongating SCFAs into butyrate and caproate is investigated. In Chapter 4 , the production of novel products, i.e. isomerised fatty acids, in microbial chain elongation with methanol is proposed and investigated. The outcome is expected to expand the product spectrum of chain elongation using organic waste. In Chapter 5, integrating methanol into the current microbial chain elongation, i.e. ethanol-based chain elongation, is explored. In such integration, a simultaneous production of multiple value-added products is expected. Furthermore, the formation of isomerised MCFAs is also postulated. 
Table 1.3. Overview of the research questions, substrates used and targeted products in each research chapter in this thesis.

\begin{tabular}{|c|c|c|c|}
\hline Chapter & Research question & Substrate & Target product \\
\hline 2 & $\begin{array}{l}\text { What are the major sources of } \\
\text { environmental impacts within } \\
\text { the life cycle of caproic acid } \\
\text { production from organic waste } \\
\text { via microbial chain } \\
\text { elongation? }\end{array}$ & $\begin{array}{l}\text { OFMSW/SFW* } \\
\text { Ethanol }\end{array}$ & $\begin{array}{l}\text { Caproic acid (non- } \\
\text { experimental } \\
\text { result) }\end{array}$ \\
\hline 3 & $\begin{array}{l}\text { Can microbial chain } \\
\text { elongation based on methanol } \\
\text { be used to produce caproate in } \\
\text { an open mixed culture } \\
\text { condition? }\end{array}$ & $\begin{array}{l}\text { Methanol } \\
\text { Acetate }\end{array}$ & $\begin{array}{l}\text { Caproate } \\
\text { Butyrate }\end{array}$ \\
\hline 4 & $\begin{array}{l}\text { Can isomerised fatty acids be } \\
\text { produced from organic waste } \\
\text { via microbial chain elongation } \\
\text { based on methanol? }\end{array}$ & $\begin{array}{l}\text { Methanol } \\
\text { Acetate/SFW }\end{array}$ & Isobutyrate \\
\hline 5 & $\begin{array}{l}\text { Can microbial chain } \\
\text { elongation based on methanol } \\
\text { be integrated into the existing } \\
\text { caproate production chain? }\end{array}$ & $\begin{array}{l}\text { Methanol } \\
\text { Ethanol } \\
\text { Acetate } \\
\text { butyrate }\end{array}$ & $\begin{array}{l}\text { Caproate } \\
\text { Isobutyrate } \\
\text { Isomerised } \\
\text { MCFAs }\end{array}$ \\
\hline
\end{tabular}

*OFMSW: organic fraction of municipal solid waste; SFW: supermarket food waste. 
General introduction

1 


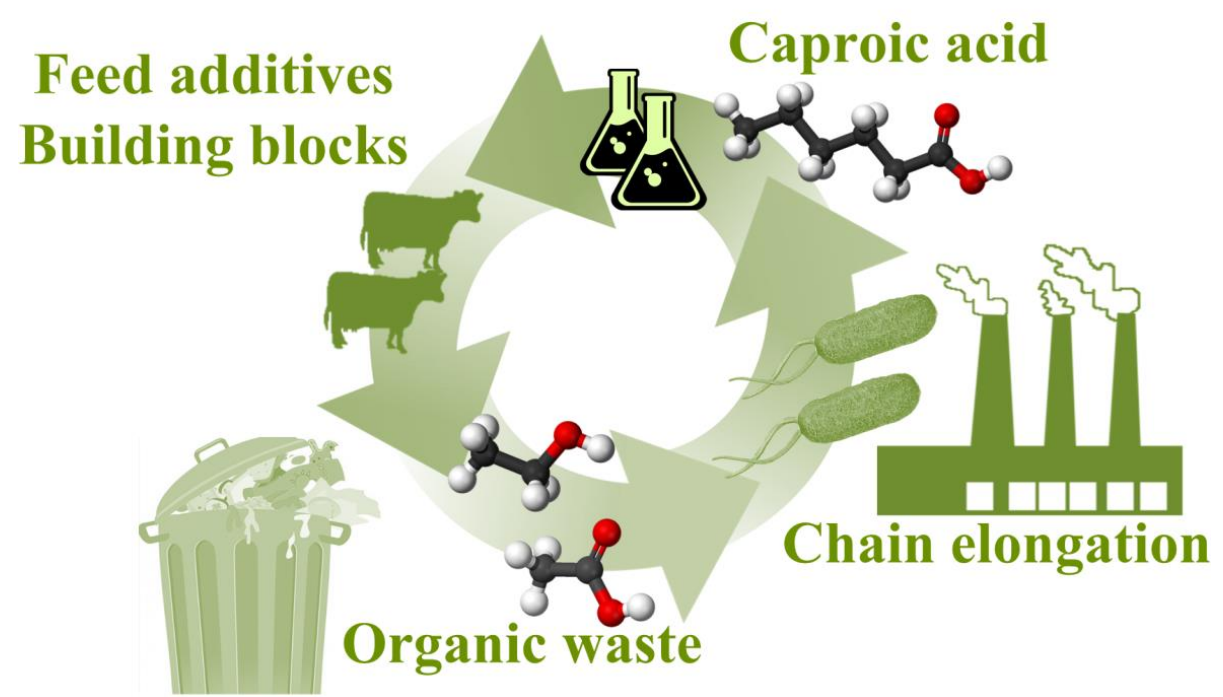




\title{
Chapter 2. Life Cycle Assessment (LCA) of microbial chain elongation
}

\begin{abstract}
Caproic acid is an emerging platform chemical with a range of diverse applications. Recently, a novel biorefinery process, i.e. microbial chain elongation, was developed to convert organic waste and ethanol into renewable caproic acids. In the coming years, this process may become commercialised, as well as continuing to improve on the basis of numerous ongoing technological and microbiological studies. This study aims to analyse the environmental performance of caproic acid production from organic waste via microbial chain elongation at this current, early stage of technological development. To this end, a Life Cycle Assessment (LCA) was performed to evaluate the environmental impact of producing $1 \mathrm{~kg}$ caproic acid from organic waste via microbial chain elongation, in both a lab-scale and a pilot-scale system. Two types of organic waste were used as substrates: firstly, the organic fraction of municipal solid waste (OFMSW), and secondly, supermarket food waste (SFW). Ethanol use was found to be the dominant cause of environmental impact over the life cycle. Extraction solvent recovery was found to be a crucial uncertainty that may have a substantial influence on life-cycle impacts. We recommend that future research and industrial producers focus on the reduction of ethanol use in microbial chain elongation and improve the recovery efficiency of the extraction solvent.
\end{abstract}

A modified version of this chapter is accepted to be published in Environmental Science \& Technology as: Chen, W.S.; Strik, D. P. B. T. B.; Buisman, C. J. N.; Kroeze, C. Production of caproic acid from mixed organic waste- an environmental life cycle perspective. 


\subsection{Introduction}

The amount of organic waste produced by society is increasing, alongside a growing demand for fuels and chemicals. Currently, fuels and chemicals are mainly produced from fossil resources or from food crops such as corn, sugarcane and palm. The vast consumption of fossil resources contributes greatly to global warming and air pollution. Using food crops for fuel and chemical production may, on the other hand, compete with human food cultivation. An alternative and more sustainable feedstock is therefore needed to support our fuel and chemical consumption.

Mixed organic waste is a promising feedstock for fuel and chemical production ${ }^{61-63}$. It refers to an organic waste stream with a highly heterogeneous composition, e.g. food supply chain waste, industrial process food waste and organic fraction of municipal solid waste $(\mathrm{OFMSW})^{14,61}$. Mixed organic waste is generated in large quantities worldwide. It usually contains large amounts of readily biodegradable organic matter and various nutrients that are essential for biotechnological applications. The challenge, however, is to produce high value end-products from mixed organic waste in order to make the process more economically attractive than the current practices, e.g. anaerobic digestion to produce biogas and composting into soil amendment ${ }^{21,64}$. There is a growing interest in producing bulk chemicals from mixed organic waste. It is argued that using organic waste as substrate for bulk chemicals yields higher value products than using it for heat, electricity and fuel ${ }^{62,63,65}$. Bulk chemical production from organic waste, is even more attractive when targeting an emerging platform chemical with a relatively small and specific niche market. This is because market potential is already guaranteed and the economic competition from an established chemical process for the market is less threatening ${ }^{64}$.

Caproic acid is an emerging platform chemical that can be produced from low-grade mixed organic waste, as recently demonstrated in both lab- ${ }^{32,37-39,66}$ and pilot-scale systems, at high 
rates and specificities ${ }^{33}$. Here we refer to caproic acid in its undisassociated form, and to caproate in its dissasociated form. Caproic acid has a wide range of applications. It can be used directly as feed additives ${ }^{67}$, antimicrobials ${ }^{68}$ and plant growth promoters ${ }^{69}$. It can also be used as a precursor to various commodities including lubricants, fragrances, paint additives and pharmaceuticals ${ }^{26,33,65}$. Currently, caproic acid is produced from food crops like palm and coconut, with oils containing less than one percent of caproic acid. Although the caproic acid produced from food crops is commercially available, the low caproic acid content in these crop oils leads to a high price and a limited market. Recently, an industrially applicable caproic acid production process using mixed organic waste as a feedstock was developed and implemented, based on a microbial fermentation process, i.e. microbial chain elongation (hereinafter referred to as chain elongation) via reversed $\beta$-oxidation pathway ${ }^{41}$. In chain elongation, short chain fatty acids (SCFAs; saturated fatty acids containing less than six carbons) and ethanol are converted by microorganisms into medium chain fatty acids (MCFAs; saturated fatty acids containing six to twelve carbons). It was found that chain elongation can be performed using an open mixed culture and in a continuous production mode ${ }^{32,37}$. Moreover, the use of SCFAs, like acetate and butyrate, in chain elongation yields caproate as the most dominant end-product with a high production rate and specificity ${ }^{35,36}$. Both acetate and butyrate are the main intermediates from anaerobic degradation of mixed organic waste, like OFMSW. Ethanol addition during the anaerobic degradation of OFMSW has been shown to stimulate chain elongation of these SCFAs, as well as ensuring the production of caproate as one of the main end-products of the process ${ }^{38,} 39$. The highest caproate production rate via this process was $26 \mathrm{~g} / \mathrm{L} /$ day with a concentration up to $12.6 \mathrm{~g} / \mathrm{L}$, which approximates the solubility of caproate and is advantageous to the downstream processes. A caproic acid production process using mixed organic waste and ethanol was thus developed. Four factors, namely the high caproate concentration, the high caproate 


\section{Chapter 2}

production rate, the use of a mixed organic waste and the possibility to use an open mixed culture makes this caproic acid production process attractive and industrially applicable. Thus, a spin-off company from Wageningen University, ChainCraft BV (Amsterdam), has developed this proven technology into a pilot-scale system that continuously converts food processing waste and ethanol into economically viable caproic acid.

Continuous caproate production via chain elongation was demonstrated for the first time in $2011^{32}$. Since then, several studies were completed to promote MCFA production from lowgrade waste via chain elongation. Most of these studies addressed the substrate range $\mathrm{e}^{38-40,43 \text {, }}$ 44, 70-72, the bioprocessing ${ }^{35-37,66,73}$, the microbiology $y^{70,74}$ and the downstream processes ${ }^{75-77}$. Recent review articles on chain elongation also focus mostly on these $\operatorname{aspects}^{33}, 34$. Surprisingly, the environmental performance of this "sustainable" bioprocess has not been addressed in any of these studies. Analysing the environmental sustainability of an emerging technology during its early stages is beneficial, not only for orienting the future technological development towards an improved environmental performance, but also for supporting decision-making during the implementation or process design stages ${ }^{60}$. An assessment providing the environmental perspective of this emerging technology will be of use for the development, implementation and commercialisation in the near future.

This study, therefore, aims to analyse the environmental performance of caproic acid production from organic waste via chain elongation at this early stage of technology development. To this end, an early-stage Life Cycle Assessment (LCA) was performed to quantify the environmental impact associated with the caproic acid production based on the existing chain elongation business case, i.e. caproic acid production from mixed organic waste and ethanol. The result may help to identify environmental impact "hot-spots" within the entire life cycle of caproic acid production from organic waste, or provide a benchmark for comparison with other existing processes ${ }^{60}$. The potential outreach of the LCA outcome 
may provide environmental sustainability as an additional perspective for orienting future research on chain elongation as well as providing a basis for strategic improvement advice, which could prove vital to industrial producers of caproic acid.

\subsection{Methodology}

\subsubsection{Life cycle assessment (LCA).}

The goal of this LCA is to quantify the environmental impact associated with caproic acid production from mixed organic waste via chain elongation. An attributional LCA was selected based on existing guidances ${ }^{78,79}$. The functional unit (f.u.) is $1 \mathrm{~kg}$ of caproic acid production (Purity $>99 \%$ ) from mixed organic waste and ethanol via chain elongation. A gate-to-product life cycle of the caproic acid production via chain elongation is assessed. The gate-to-product life cycle starts from the organic waste arriving at the caproic acid production site and ends with the product, i.e. 1 f.u. of caproic acid leaving the caproic acid production site. Environmental impacts associated with all waste treatments during the defined gate-toproduct life cycle are included in the assessment. The emissions and environmental impacts associated with the generation of mixed organic waste, which is used as a feedstock, are not considered. The organic waste used in the present study is a low-grade mixed organic waste, and it exists regardless of whether the caproic acid would have been produced or not. The environmental impacts associated with the generation of the organic waste should, therefore, be allocated to the processes or products from which the waste is generated. In some cases, the environmental impacts are allocated to the waste if the waste is considered a by-product; for example, glycerol as a by-product from biodiesel production ${ }^{80}$. This study differs from these by-product cases as the organic waste used in the present study is a low-grade waste with a mixed and complex composition that cannot be considered as a by-product. However, in the future, when more and more waste-to-resource technologies are implemented specifically for mixed organic waste, this waste stream may have to be considered as a by- 


\section{Chapter 2}

product, and part of the environmental impacts of the generation of mixed organic waste need to be allocated to these low-grade by-products.

Data for the Life Cycle Inventory (LCI) were collected from, in order of preference, internal data that were published ${ }^{30,39}$, existing literature ${ }^{26,41}$, internal data that were not published and personal communications with industrial producers and experts (see Table S2.1 in the Support Information 2 (SI2) for details). The information required to carry out the Life Cycle Impact Assessment (LCIA) was sourced from literature and the Ecoinvent 3 database $^{81}$. The characterization method used was CML-IA baseline V3.02/EU25. Global Warming Potential (GWP; $\mathrm{CO}_{2}$-equivalent/f.u.), Eutrophication Potential (EP; $\mathrm{PO}_{4}{ }^{3-}$ equivalent/f.u.) and Acidification Potential (AP; $\mathrm{SO}_{2}$-equivalent/f.u.) were the selected impact categories based on the existing guidance ${ }^{82}$ and data availability. An overview of the data used in the LCIA is available in Table S2.2 (see SI2).

\subsubsection{Production system description and cases.}

The assessed system consists of six main processes (Figure 2.1), which starts with the mixed organic waste and ends at the production of caproic acid. Three types of organic waste were used for caproic acid production via chain elongation ${ }^{33}$, including the organic fraction of municipal solid waste $(\mathrm{OFMSW})^{30,38,77}$, the yeast-fermentation beer from the corn kernel-toethanol industry $^{37,66}$ and supermarket food waste (SFW) from the food residue processing industry (unpublished data). The SFW has been applied in a pilot-scale system (ChainCraft B.V., Amsterdam); the other two were only applied in lab-scale bioreactor systems ${ }^{33}$. In this study, OFMSW and SFW are selected to be assessed because we aim to use a low-grade, mixed and geographically wide-spread waste stream as the feedstock. Moreover, a large quantity of internal data using OFMSW and SFW for caproic acid production via chain elongation is available in our institute. Three cases were developed based on the process data we possess (see Table S2.3). They are the lab-scale system using OFMSW (Case LO), the 
lab-scale system using SFW (Case LS) and the pilot-scale system using SFW (Case PS). Part of the Case PS was simulated using the data from Case LS considering the available data from the pilot plant. A detailed description of the three cases can be found in Table S2.3 in SI2.

The organic waste, i.e. OFMSW or SFW, enters the biological acidification (BAc) process without any pre-treatment. In BAc, an undefined, mixed culture microbiome hydrolyses the organic solids into soluble organic matters and further degrades the soluble organic matters into basic building blocks like SCFAs, $\mathrm{CO}_{2}$ and $\mathrm{H}_{2}$. These basic building blocks are essential substrates for the next process, chain elongation (CE). Two types of BAc were used in different cases depending on the water content of the organic waste. A dry anaerobic acidification was applied to $\mathrm{OFMSW}^{30}$, and a wet anaerobic acidification was applied to SFW. The effluent of BAc consists of the broth that enters CE and the solid residues that are disposed of. The disposed pellets, together with other solid waste generated in Case LO and LS, are assumed to be incinerated. According to ChainCraft B.V., in Case PS, anaerobic digestion was applied to recover energy from all solid residue generated during the life cycle of caproic acid production.

In $\mathrm{CE}$, another undefined mixed culture microbiome elongates the SCFAs with the externally added ethanol into caproate. Corn-based bioethanol was used in CE, and the amount of ethanol dose required was derived from the existing literature ${ }^{39}$ (for Case LO) or internal experimental data (for Case LS and PS). $\mathrm{CO}_{2}$ was continuously supplied during the entire CE process to sustain the microbial growth ${ }^{39,}{ }^{41}$. NaOH was continuously added to maintain an optimal $\mathrm{pH}$ for caproate production using an automated $\mathrm{pH}$ controller, as a lower $\mathrm{pH}$ could lead to product toxicity. The amount of $\mathrm{NaOH}$ required was estimated by calculating the amount of proton formation, based on the amount of caproate produced and the microbial metabolism of Clostridium kluyveri, a known chain-elongating bacterium ${ }^{26,41}$. 


\section{Chapter 2}

The effluent of CE contains about $12.6 \mathrm{~g} / \mathrm{L}$ caproate, and the caproate yield is about $0.5 \mathrm{~g}$ $\mathrm{COD} / \mathrm{gCOD}_{\text {waste+ethanol }}{ }^{39}$. Biogas is produced during both $\mathrm{BAc}$ and $\mathrm{CE}$, and, currently, the resulting biogas is not collected for any application. Considering the origin of the feedstock, the $\mathrm{CO}_{2}$ emission in the biogas during $\mathrm{BAc}$ and $\mathrm{CE}$ could be attributed to biogenic carbon emission that does not contribute to global warming. $\mathrm{CH}_{4}$ emission during $\mathrm{BAc}$ and $\mathrm{CE}$, on the other hand, is accounted as a Greenhouse Gas (GHG) emission.

The effluent of $\mathrm{CE}$ goes through downstream processes; including the liquid-solid separation (LSS), the chemical acidification (CAc), the liquid-liquid extraction (LLEx) and distillation (Ds). LSS is carried out using a centrifuge and the pellet is disposed of. The supernatant enters CAc in which hydrochloric acid is added to lower the $\mathrm{pH}$ of the supernatant to 4.9. Low $\mathrm{pH}$ enhances the protonation of caproate into caproic acid which can then be extracted. This extraction takes place by mixing the solvent with the effluent from CAc. During the mixing, part of the caproic acid transfers from the aqueous phase to the solvent phase. The fraction of the caproic acid entering the solvent phase is based on the distribution coefficient. Several extraction solvents were examined for their distribution coefficients in the literature, e.g. ethyl caproate ${ }^{22}$ and petroleum ether ${ }^{22}$. To our best knowledge, the life-cycle impact of both ethyl caproate and petroleum ether have not been reported so far. The life-cycle impact of ethyl acetate is used for simulating the life-cycle impact of ethyl caproate. Ethyl acetate itself was also used for the extraction of propionic acid in a biorefinery system that is similar to the present study ${ }^{80}$. After LLEx, the solvent phase is distilled to recover both the caproic acid in a high purity form as well as the reusable solvent, while the distillate enters the wastewater treatment system.

\subsubsection{Sensitivity analysis and comparison to other studies}

A sensitivity analysis was performed to evaluate the sensitivity of the life-cycle environmental impacts of the alternative materials/data sources. The analysis was carried out 
by using alternative data sets (see SI2 Table S2.4 for details). The three study cases, i.e. Lo, LS and PS, were used as baselines for the sensitivity analysis. In addition, the results of the LCA are compared to other studies published in the literature that address the life-cycle impact of treating organic waste with mixed culture biotechnologies.

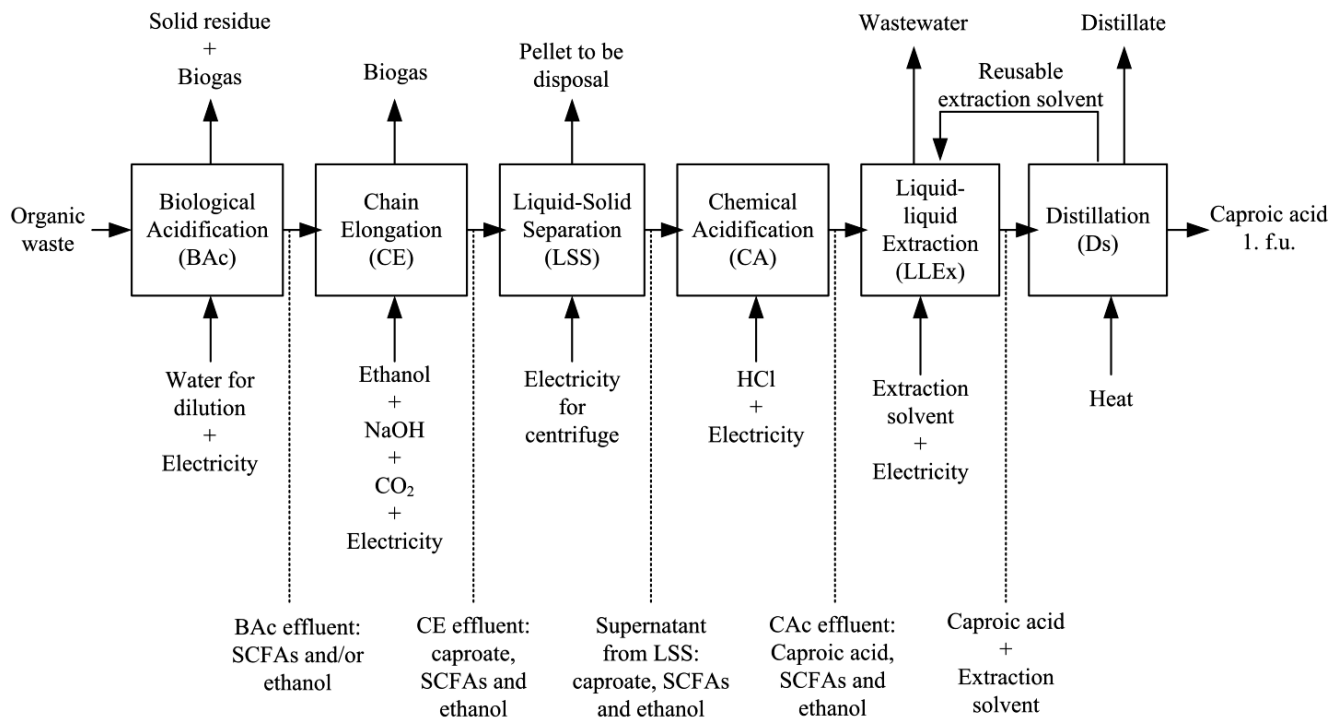

Figure 2.1. The gate-to-product life cycle of caproic acid production assessed in this study.

The life cycle starts at a mixed organic waste arriving at the caproic acid production site and ends at the caproic acid produced at the production site.

\subsection{Results and discussion}

\subsubsection{The life-cycle impacts}

Figure 2.2 shows the sum, as well as the breakdown, of the overall life-cycle impacts of caproic acid production from mixed organic waste via chain elongation. The ethanol use in chain elongation (CE) is the most dominant cause of environmental impact throughout all cases and impact categories assessed. The use of $\mathrm{NaOH}$ and $\mathrm{HCl}$ for neutralising $\mathrm{pH}$ also contributed considerably to all three impact categories assessed throughout the three cases. In 


\section{Chapter 2}

Case LO (OFMSW in lab-scale system) and LS (SFW in lab-scale system), the extraction solvent used in the liquid-liquid extraction (LLEx) process was the most dominant environmental impact source. In Case LO, the solid waste management had a considerably higher contribution to the life-cycle impact than Case LS and PS (SFW in pilot-scale system), due to the type of organic waste used as a substrate and the type of biological acidification (BAc) used.

A large quantity of ethanol (1.8 kg/f.u. in Case LO and $1.5 \mathrm{~kg} / \mathrm{f} . \mathrm{u}$. in Case LS and PS) was added during $\mathrm{CE}$ as an essential substrate for high-rate, caproic acid production from organic waste via chain elongation. It serves as both a carbon source and as an electron donor, which provides energy for the chain-elongating microorganisms ${ }^{33}$. The environmental impacts of the added ethanol account for at least $20 \%$ of the total life-cycle impacts for all impact categories in all cases. More than half of the impacts of the added ethanol originate from the feedstock production, i.e. production of corn grains ${ }^{83}$. The use of $1 \mathrm{~kg}$ of corn-based bioethanol gives $1.6 \mathrm{~kg} \mathrm{CO}_{2}$-eq GWP, of which $0.9 \mathrm{~kg} \mathrm{CO}_{2}$-eq GWP (56\%) resulted from the production and transportation of the corn grain. For AP and EP, $75 \%$ and $78 \%$ of the overall impact of $1 \mathrm{~kg}$ of corn-based bioethanol are associated with the production of corn grains respectively. The high $\mathrm{AP}$ and $\mathrm{EP}$ are related to nitrogen and phosphorus emissions from the soil due to fertiliser application during cultivation ${ }^{84}$. 


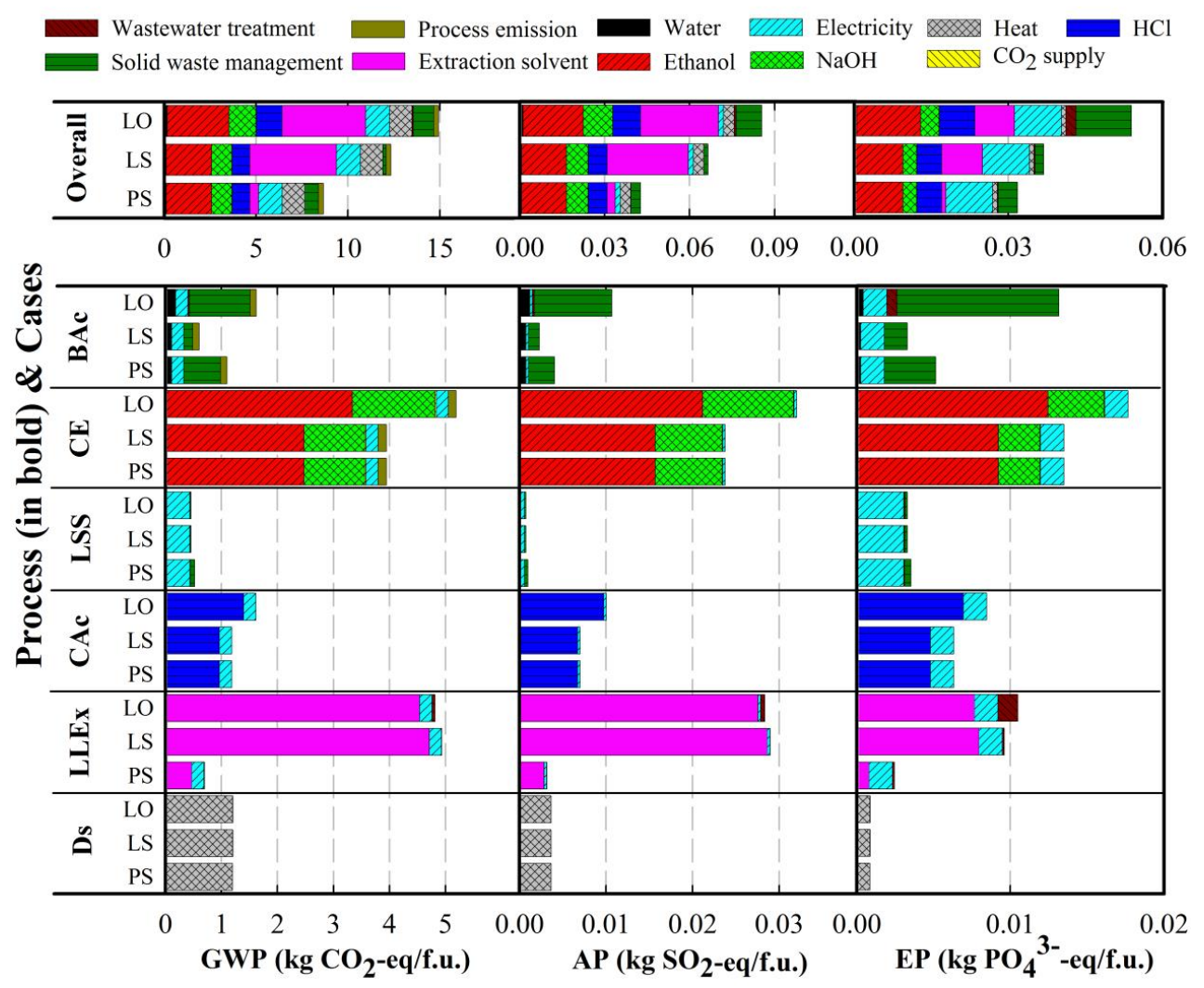

Figure 2.2. The gate-to-product life cycle impact of $1 \mathrm{~kg}$ of caproic acid produced through chain elongation. Results are shown for Global Warning Potential (GWP), acidification potential (AP) and eutrophication potential (EP) for each process used (in bold; see Figure 2.1) in each case (Case LO, LS and PS; see section 2.2.2 and Table S2.3).

There are three potential strategies for reducing the environmental impacts of ethanol addition. As the ethanol production system is beyond the system boundary of the present study, we therefore only discuss improvement strategies that can be implemented within our system boundaries. The first strategy is to stimulate the in-situ ethanol formation within the organic waste during BAc. The ethanol consumption (1.8 kg/f.u.) and the ethanol concentration $(19.3 \mathrm{~g} / \mathrm{L})$ required for caproic acid production in all cases were assumed to be 


\section{Chapter 2}

the same. However, the amount of ethanol addition in Cases LS and PS is lower than that in Case LO, because there is in-situ ethanol formation (up to $5.3 \mathrm{~g} / \mathrm{L}$; around $0.5 \mathrm{~kg} / \mathrm{f}$.u.) during the BAc of SFW. In contrast, during the BAc of OFMSW, there is hardly any in-situ ethanol production $^{39}$. The higher ethanol addition in CE in Case LO consequently increased the lifecycle impacts. Substrate composition ${ }^{85}, \mathrm{pH}^{86}$ and headspace hydrogen partial pressure ${ }^{85}$ are parameters that can affect the in-situ ethanol production during $\mathrm{BAc}^{87,88}$. In SFW, there were likely more carbohydrates that are easily fermented into ethanol compared with OFMSW ${ }^{89}$. During the BAc of OFMSW and SFW, the pH was similar (between 5 to 5.5), and the hydrogen partial pressure in the headspace were not reported. Potential strategies that can stimulate in-situ ethanol production during BAc may be further investigated.

The second strategy for reducing the ethanol addition is to improve the accuracy of the ethanol dose. Based on the currently known stoichiometry of the chain elongation reaction ${ }^{37}$, ${ }^{41}, 2.4$ moles of ethanol and 1 mole of acetate is used to produce 1 mole of caproate. This means that the production of $1 \mathrm{~kg}$ of caproic acid, i.e. 1 f.u., requires $0.9 \mathrm{~kg}$ of ethanol (pure) in the most ideal condition, which is half of the totally available ethanol (i.e. the sum of the added ethanol and in-situ ethanol formation) in all study cases. In other words, about half of the ethanol supply in CE in this study does not end in the final product. The ethanol that is not used for chain elongation to caproic acid either remains in the CE effluent, which is wasted, or it is consumed by other reactions during CE, e.g. excessive ethanol oxidation to acetate and hydrogen that is not associated with chain elongation. This means that there is still room for reducing the ethanol addition via precisely controlling the ethanol dose during CE. The third strategy is to employ a substitute for ethanol in CE. Hydrogen ${ }^{32}$, renewable electricity $^{42}$, methanol ${ }^{90,91}$, lactate ${ }^{43,44}$ and ethanol from more renewable sources, e.g. syngas fermentation broth, have been used to substitute the current ethanol use in $\mathrm{CE}^{40}$, though they are still in an early stage of development. 
The use of $\mathrm{NaOH}$ and $\mathrm{HCl}$ for neutralising $\mathrm{pH}$ contributed considerably to all three impact categories assessed throughout the three cases. This is mainly due to the electricity used during the $\mathrm{NaOH}$ and $\mathrm{HCl}$ production process, i.e. the electrolysis of brine or so-called chloralkali process. The use of $\mathrm{NaOH}$ during the anaerobic fermentation of organic waste for propionic acid production was reported to contribute $11 \%$ of the overall $\mathrm{GWP}^{80}$, which is similar to the result of this study. The use of this base cannot be omitted if a high rate caproic acid production is targeted, as the $\mathrm{pH}$ drop induced by caproic acid accumulation inhibits the microbial activities. Continuous removal of caproic acid from the fermentation broth inside the $\mathrm{CE}$ bioreactor, e.g. via an in-line liquid-liquid membrane extraction, may help reduce the use of $\mathrm{NaOH}^{37}$. A combination of the in-line extraction system and an in-line membrane electrolysis for the caproic acid recovery can even further avoid the external supply of $\mathrm{HCl}$ in the chemical acidification (CAc) process, as the protons required to extract the caproic acid are produced in the electrochemical system ${ }^{92}$. However, such a system would require several membranes, which are manufactured via energy-intensive processes ${ }^{93}$ and have to be replaced regularly. The trade-off between the additional impacts due to the use of membranes and the impact reduction due to the avoided use of $\mathrm{NaOH}$ and $\mathrm{HCl}$ should be carefully evaluated.

Case PS has a much lower life-cycle impact compared with Case LO and LS, mainly due to the recovery of a large portion of the extraction solvent (99\% in Case PS versus. $90 \%$ in Case LO and LS). In the lab-scale system, the extraction solvent loss during the LLEx and distillation (Ds) processes was assumed to be about $5 \%$ of the total added solvent for each of the two processes. A $90 \%$ solvent recovery was, therefore, assumed. This estimation was made to give the worst-case scenario. Based on this assumption, the extraction solvent consumption was the largest contribution to the life-cycle impacts in Cases LO and LS. This was due to both the high demand for solvent replenishment $(1.7 \mathrm{~kg} / \mathrm{f} . \mathrm{u}$.) and the high lifecycle impacts of the extraction solvent ${ }^{81}$. According to ChainCraft B.V., a solvent recovery 


\section{Chapter 2}

efficiency up to $99 \%$ is feasible in the pilot-scale system. A similar solvent recovery efficiency, i.e. $98.5 \%$, was also assumed to be feasible in a previous study on a similar downstream process system that recovers propionic acid from the fermentation broth $^{80}$. If the solvent recovery efficiency can be up to $99 \%$, the life-cycle impacts as well as the impact generated by the solvent consumption can be reduced significantly, as shown in the impact of LLEx in Case PS (Figure 2.2). However, the actual environmental impact that arose from the solvent consumption is quite uncertain and requires further investigation. This is because the distillation has not been performed in the lab-scale system, and the LLEx performed in the lab-scale system is not yet well developed. In addition to the quantity of the solvent consumed, the data quality of the solvent is another uncertainty. In the current study, the lifecycle impact of ethyl caproate was simulated by using the life-cycle impact data of ethyl acetate derived from Ecoinvent 3. In Ecoinvent 3, the acetate required for manufacturing ethyl acetate is mainly produced from syngas, which is derived from the partial combustion of heavy fuel oil or coal, which are fossil-based. This combustion process contributed a large portion of the life-cycle GWP and AP of ethyl acetate. However, more environmentally sustainable acetate manufacturing processes using $\mathrm{CO}_{2}$ or organic waste as substrates are under development, the use of which may reduce the life-cycle impact of ethyl acetate considerably $^{94}$. Alternatively, the environmental sustainability of different types of extraction solvents; e.g. biodiesel derived from residual kitchen oil $^{77}$ or extraction process, e.g. an inline liquid-liquid membrane extraction ${ }^{37,66}$ an in-line membrane electrolysis ${ }^{75}$ and an electrodialysis with a bipolar membrane ${ }^{85}$, that have been applied to recover caproic acid should also be investigated. However, these separation techniques have only been demonstrated in lab-scale systems.

The overall life-cycle impact of using OFMSW as a feedstock (i.e. Case LO) is higher than using SFW as the feedstock (i.e. Case LS and PS) throughout the three impact categories 
assessed. This is mainly due to the large quantity of solid waste that remains after the BAc process and the higher ethanol addition in CE in Case LO. A large quantity of solid waste (33.3 kg/f.u.) remained after the dry anaerobic digestion of OFMSW, due to the high lignocellulosic content in the OFMSW (90\% volume-to-volume garden waste) that was difficult to be biologically degraded. The lignocellulosic fraction of the OFMSW may have to be pre-treated to be effectively degraded, which was not employed in the previous chain elongation study (from which we obtained the data). However, the application and selection of the pre-treatment methods have to be carefully evaluated as the application of pretreatments before anaerobic digestion (BAc in this study) can increase the life-cycle impact, especially the eutrophication potential as well as the life-cycle cost ${ }^{95}$.

\subsubsection{Sensitivity analysis}

Corn-based bioethanol was reported to have a poorer environmental sustainability, especially in GWP and nutrient use efficiency, compared with other crops like sugarcane ${ }^{96}$. Sugarcane bioethanol is a potential alternative to corn ethanol as it is already commercially available at a large production scale ${ }^{97}$. In the SA (Table 2.1), the use of sugarcane bioethanol in CE reduces the life-cycle GWP considerably. Sugarcane bioethanol was reported to have a considerably lower GHG emission rate compared with other crop-based bioethanol from different feedstocks ${ }^{96,98}$. This is likely due to the climate conditions where it grows and the properties of the crops. It is reported that, in general, temperate annual crops (like corn) have a lower productivity and net energy production compared with perennial crops growing in tropical zones (e.g. sugarcane in Brazil) ${ }^{96,99}$. Given the same amount of plantation area, sugarcane in the tropical zone could yield up to two times more bioethanol than corn in the temperate region $^{99}$. After replacing corn ethanol with sugarcane ethanol, the life-cycle GWP of all cases decreased significantly $(>12 \%)$, while the life-cycle AP and EP remained similar. 


\section{Chapter 2}

When using a fossil-based ethanol (produced via hydration of ethylene) instead of a cropbased bioethanol, the environmental impact is lower, especially for the AP and EP (Table 2.1). The reduction in AP and EP by using fossil-based ethanol was anticipated, as this had already been reported in several previous studies, mainly due to nutrient leaching and fertiliser application during the crop cultivation ${ }^{10,97}$. On the other hand, GWP of fossil ethanol is lower than that of corn ethanol. This corresponds to the outcome of previous studies ${ }^{96,97}$ but is intuitively contradictory. A key reason for this may be the exclusion of the end-of-life of ethanol. When the end-of-life of ethanol is not included, fossil ethanol has a similar or, in some cases, lower life-cycle GWP compared to other crop bioethanol. However, when the end-of-life is included, bioethanol could have a lower GWP than fossil ethanol, because part of the carbon emission could be counted as biogenic carbon emission ${ }^{97}$. For fossil ethanol, all carbon emissions in the use phase and the end-of-life phase are accounted for as non-biogenic carbon that contributes to the GWP, regardless of the purpose of the ethanol use. However, for crop bioethanol, the purpose of the ethanol use has a substantial influence on the GWP associated with the end-of-life of bioethanol. If bioethanol is used as a fuel and combusted, most of the carbon emitted is in the form of biogenic $\mathrm{CO}_{2}$ that does not have any GWP. In the case that bioethanol is not combusted but used as an additive or precursor to chemicals, e.g. caproic acid in the present study, part of the bioethanol will end up in the water phase and eventually form methane via biological degradation (e.g. anaerobic digestion), which cannot be counted as a biogenic emission and thus contributes to GWP ${ }^{100}$. In the present study, the end-of-life of the ethanol is not yet included, as the end-of-life of caproic acid is not within the system boundary (due to the various potential applications of caproic acid). In the future, when the life cycle of caproic acid production via chain elongation is assessed for a specified application, the feedstock as well as the end-of-life of the ethanol should be carefully addressed. 
Lignocellulosic bioethanol is an alternative ethanol source that is becoming increasingly available in Europe ${ }^{50}$. As shown in Table 2.1, the use of lignocellulosic bioethanol produced from grass has a clear reduction on the life-cycle GWP, but the use of it increases the lifecycle AP. The higher AP could be attributed to the steam (i.e. heat) used to pre-treat the grass to yield higher ethanol production. The chemical, e.g. sulfuric acid, used for pre-treating lignocellulosic biomass could also be one of the main causes for the high life-cycle $\mathrm{AP}^{101}$. Overall, based on the present study, the use of lignocellulosic bioethanol does reduce the lifecycle GWP but not the life-cycle AP and EP.

Reducing ethanol addition is another potential improvement strategy, as discussed in section 2.3.1. Based on the stoichiometry of the chain elongation reaction, the maximum possible ethanol reduction (in the form of a $95 \%$ ethanol solution) is about $1 \mathrm{~kg} / \mathrm{f} . \mathrm{u}$. for all cases. This reduction in ethanol dose leads to a substantial reduction of all life-cycle impacts throughout all cases, especially in Case PS where the ethanol use dominates the life-cycle impacts. The ethanol use efficiency, as well as the possible reduction on ethanol addition in $\mathrm{CE}$, have not yet been specifically addressed in previous studies on chain elongation, to authors' best knowledge. Regarding the potentially substantial reduction of the life-cycle impacts, it is advised to study the maximal feasible reduction on ethanol addition.

The use of mineral oil as the extraction solvent was investigated in internal experiments, which had a similar extraction performance as the ethyl caproate (Data not shown). In SA, the use of mineral oil decreases all life-cycle impacts in all cases, except for the life-cycle AP in Case PS. Mineral oil is a by-product from oil refinery with a low economic value. Due to its low economic value, the life-cycle impacts allocated to mineral oil is low compared with other products produced from the oil refinery. This may be the reason why the use of mineral oil in chain elongation leads to a lower life-cycle impact. However, it should be kept in mind that mineral oil is still a fossil-based material which is considered a non-renewable resource. 


\section{Chapter 2}

Moreover, even in the case that mineral oil is used as the recovery solvent, the contribution of solvent use to the life-cycle impacts in Cases LO and LS is still considerably high. The solvent recovery efficiency still has a greater influence on the environmental impact associated with the use of extraction solvent. 
Table 2.1. Overview of the results of the sensitivity analysis. The change of the life cycle impact due to the usage of alternative material/dataset is presented in terms of the percentage of the life cycle impact derived from the baseline cases.

\begin{tabular}{|c|c|c|c|c|c|c|c|c|c|}
\hline & \multicolumn{3}{|c|}{ Case LO } & \multicolumn{3}{|c|}{ Case LS } & \multicolumn{3}{|c|}{ Case PS } \\
\hline & GWP & AP & EP & GWP & $\mathrm{AP}$ & $\mathrm{EP}$ & GWP & $\mathrm{AP}$ & EP \\
\hline $\begin{array}{l}\text { Baseline (kg } \\
\mathrm{CO}_{2} / \mathrm{SO}_{2} / \mathrm{PO}_{4}{ }^{3-} \\
\text { eq per f.u.) }\end{array}$ & 14.6 & 0.08 & 0.05 & 12.34 & 0.07 & 0.04 & 8.66 & 0.04 & 0.03 \\
\hline $\begin{array}{l}\text { Use of } \\
\text { sugarcane } \\
\text { bioethanol, } \\
\text { Brazil }\end{array}$ & $\downarrow 12 \%$ & $\downarrow 1 \%$ & $\uparrow 1 \%$ & $\downarrow 12 \%$ & $\downarrow 1 \%$ & $\uparrow 1 \%$ & $\downarrow 17 \%$ & $\downarrow 2 \%$ & $\uparrow 1 \%$ \\
\hline $\begin{array}{l}\text { Use of fossil- } \\
\text { based ethanol, } \\
\text { Europe }^{\dagger}\end{array}$ & $\downarrow 5 \%$ & $\downarrow 14 \%$ & $\downarrow 15 \%$ & $\downarrow 5 \%$ & $\downarrow 15 \%$ & $\downarrow 17 \%$ & $\downarrow 7 \%$ & $\downarrow 23 \%$ & $\downarrow 20 \%$ \\
\hline $\begin{array}{l}\text { Use of } \\
\text { lignocellulosic } \\
\text { bioethanol } \\
\text { (from grass), } \\
\text { Europe }\end{array}$ & $\downarrow 10 \%$ & $\uparrow 5 \%$ & $0 \%$ & $\downarrow 9 \%$ & $\uparrow 5 \%$ & $0 \%$ & $\downarrow 13 \%$ & $\uparrow 8 \%$ & $0 \%$ \\
\hline $\begin{array}{l}\text { Precise control } \\
\text { of ethanol dose } \\
\text { during CE ( } 50 \% \\
\text { ethanol dose } \\
\text { reduction) }\end{array}$ & $\downarrow 10 \%$ & $\downarrow 11 \%$ & $\downarrow 10 \%$ & $\downarrow 13 \%$ & $\downarrow 15 \%$ & $\downarrow 16 \%$ & $\downarrow 19 \%$ & $\downarrow 24 \%$ & $\downarrow 19 \%$ \\
\hline $\begin{array}{l}\text { Mineral oil as } \\
\text { the extraction } \\
\text { solvent }\end{array}$ & $\downarrow 11 \%$ & $\downarrow 2 \%$ & $\downarrow 12 \%$ & $\downarrow 13 \%$ & $\downarrow 2 \%$ & $\downarrow 17 \%$ & $\downarrow 2 \%$ & $0 \%$ & $\downarrow 2 \%$ \\
\hline $\begin{array}{l}\mathrm{NaOH} \\
\text { alternative } \\
\text { data }^{102}\end{array}$ & $\downarrow 5 \%$ & $\downarrow 12 \%$ & $\downarrow 7 \%$ & $\downarrow 4 \%$ & $\downarrow 11 \%$ & $\downarrow 7 \%$ & $\downarrow 6 \%$ & $\downarrow 17 \%$ & $\downarrow 8 \%$ \\
\hline
\end{tabular}




\subsubsection{Comparison with other studies}

Comparing the LCA outcome of this study with other competing technologies is of use to benchmark the technology assessed in this study. There are three ways to compare the outcome of this LCA, i.e. the life-cycle impact of caproic acid, with other studies. One is to compare it to other LCAs for caproic acid production processes using feedstocks other than organic waste. However, such LCAs are not available to authors' current knowledge. The second is to compare it to LCAs of other novel products from organic waste via emerging biotechnologies, e.g. polyhydroxyalkanoates (PHA). The third and final way is to compare it to LCAs for other current ways of organic waste treatment (composting, anaerobic digestion, combustion, etc.). Henceforth, we use PHA and anaerobic digestion as two examples for comparing and reflecting our LCA.

Gurieff and Lant (2007) reported an LCA study for PHA that can be used to compare with the present study ${ }^{103}$. The other LCA studies for PHA mostly used either a more homogenous waste stream or a pure-culture bioprocess ${ }^{104}$. In Gurieff and Lant (2007), a 20 g-COD/L mixed wastewater stream from the food industry was used to produce PHAs, using a mixed culture under a non-sterile condition. They report that the life-cycle GWP of PHA from a mixed wastewater stream is about $20.4 \mathrm{~kg} \mathrm{CO}$-eq $/ \mathrm{kg}$ PHA or $3.92 \mathrm{~kg} \mathrm{CO}$-eq/kg-COD feed (excluding the environmental benefits of displacing fossil-based polymers). In Case PS of this study, the life-cycle GWP of caproic acid is about $8.7 \mathrm{~kg} \mathrm{CO}_{2}$-eq $/ \mathrm{kg}$ caproic acid or 2.3 $\mathrm{kg} \mathrm{CO}$-eq/kg-COD feed (based on the COD in the SFW), which is lower than that of PHA reported by Gurieff and Lant (2007). The main reason to exclude the environmental benefits of PHA is due to the uncertain application of caproic acid. When the environmental impact of displacing an existing fossil-based polymer is accounted for, the PHA production from the mixed wastewater has a negative environmental impact, i.e. it gains environmental benefits while treating the wastewater. It is therefore recommended to identify a specific application of caproic acid in future LCA studies to effectively evaluate the environmental credentials for 
caproic acid. An example can be the use of caproic acid as a feed additive, which improves the feed conversion efficiency and the health of livestock ${ }^{67}$. In this case, caproic acid can obtain environmental credentials by saving animal feed due to the higher feed conversion efficiency. Another interesting fact of this comparison (caproic acid versus PHAs) is the respective environmental impact hot-spots of both systems; namely the ethanol use in the caproic acid production and the electricity use in the PHA system for the downstream processes $^{103,104}$. As the environmental impacts from electricity use can be significantly reduced by introducing renewable electricity, the life-cycle impact of PHA production via a mixed culture bioprocess can be significantly reduced by employing a cleaner electricity ${ }^{104}$. In contrast, ethanol use in caproic acid production is currently inevitable, though the ethanol dose may be significantly reduced. The development of alternative electron donors for chain elongation is of importance to improve the life-cycle environmental performance of the caproic acid production via chain elongation.

Anaerobic Digestion (AD) has been implemented for treating organic waste, which yields biogas as a by-product. According to a recent LCA of a large-scale AD on OFMSW, treating $1 \mathrm{~kg}$ of municipal organic waste via $\mathrm{AD}$ generates $0.056 \mathrm{~kg} \mathrm{CO}_{2}$-eq $/ \mathrm{kg}$ OFMSW (recalculated from Evangelisti et al. $(2014)^{105}$ ), excluding the avoided environmental burden of the produced biogas. Assuming that the OFMSW used in this AD study has a similar COD concentration as the OFMSW used in our study (0.55 $\mathrm{kgCOD} / \mathrm{kg}$ OFMSW), the environmental impact of this large-scale AD treating OFMSW is about $0.1 \mathrm{~kg} \mathrm{CO}$-eq $/ \mathrm{kg}$

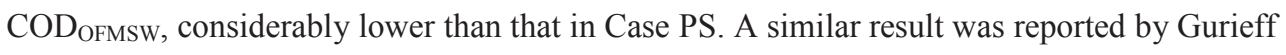
and Lant (2007) when they compared the PHA production and AD for treating a mixed food industrial wastewater without crediting the end-products ${ }^{103}$. The system scales may be a factor contributing to the significant difference in the environmental performances of these bioprocesses. It is known that lab-scale and pilot-scale systems, e.g. this study and the PHA 


\section{Chapter 2}

production $^{103}$, may have lower yields than commercial-scale systems such as large-scale $\mathrm{AD}^{60,105}$. On the other hand, after giving environmental credentials to the product, the PHA production appears to be a more environmentally sustainable option for managing organic waste than $\mathrm{AD}^{103}$. We anticipate the same outcome after the caproic acid is credited for the environmental impacts it avoided by using chain elongation production process.

\subsubsection{Outlook}

In this study, an LCA was performed to quantify the life-cycle impact of caproic acid production from organic waste via chain elongation. The LCA result shows that ethanol use was the most dominant source of environmental impact over the life cycle, especially when the recovery efficiency of the extraction solvent is higher, i.e. in Case PS. However, the recovery of, and the LCI data on, the extraction solvent was found to be a crucial uncertainty that can have a substantial influence on the life-cycle impact of caproic acid production. The sensitivity analysis shows that improving the precision of the ethanol dose can considerably improve the environmental performance of caproic acid production. It is recommended for future chain elongation studies and the industrial producers of caproic acid, to study the use of alternative electron donors to ethanol, and to evaluate the potential reduction of ethanol use in chain elongation without compromising the caproic acid production. The comparison of this study with other waste valorisation biotechnologies further strengthened the necessity of replacing, or reducing, ethanol use in chain elongation. Moreover it is advised, for future LCA studies to identify an application for caproic acid. This is useful as it gives caproic acid an environmental credential and enables further evaluation of its environmental performance. 


\section{List of abbreviations}

$\begin{array}{ll}\mathrm{AP} & \text { acidification potential } \\ \mathrm{BAc} & \text { biological acidification } \\ \mathrm{CAc} & \text { chemical acidification } \\ \mathrm{CE} & \text { chain elongation } \\ \mathrm{CH}_{4} & \text { methane } \\ \mathrm{CO}_{2} & \text { carbon dioxide } \\ \mathrm{CO}_{2} \text {-eq } & \text { carbon dioxide equivalent } \\ \mathrm{Ds} & \text { distillation } \\ \mathrm{EP} & \text { eutrophication potential } \\ \text { f.u. } & \text { functional unit } \\ \mathrm{GHG} & \text { greenhouse gases } \\ \mathrm{GWP} & \text { global warming potential } \\ \mathrm{HCl} & \text { hydrochloric acid } \\ \text { kg } & \text { kilogram } \\ \text { LCA } & \text { life cycle assessment } \\ \text { LCI } & \text { life cycle inventory } \\ \text { LCIA } & \text { life cycle impact assessment } \\ \text { LLEx } & \text { liquid-liquid extraction } \\ \text { LSS } & \text { liquid-solid separation } \\ \mathrm{MCFA} & \text { medium chain fatty acids } \\ \mathrm{NaOH} & \text { sodium hydroxide } \\ \mathrm{OFMSW} & \text { organic fraction of municipal solid waste } \\ \text { PO }{ }_{4}^{3-}-\text {-eq } & \text { phosphate equivalent } \\ \mathrm{SCFA} & \text { short chain fatty acids } \\ \mathrm{SFW} & \text { supermarket food waste } \\ \mathrm{SO}{ }_{2} \text {-eq } & \text { sulfur dioxide equivalent } \\ & \end{array}$




\section{Support Information for Chapter 2 (SI2)}

\section{Summary}

In this support section, information used during the performance of the Life Cycle Assessment (LCA) in the present study is documented. In Table S2.1, information used to formulate the Life Cycle Inventory (LCI) of $1 \mathrm{~kg}$ caproic acid production from organic waste via chain elongation is shown. This includes the process parameters, data sources and additional remarks/assumptions for each process involved in the life cycle. In Table S2.2, the information required to calculate the Life Cycle Impact Assessment (LCIA) of $1 \mathrm{~kg}$ caproic acid production from organic waste via chain elongation is documented. Note that the LCIA data derived from the Ecoinvent 3 database was altered in some cases to fit the actual condition of the assessed system. An example is the $\mathrm{CO}_{2}$ emission in the solid waste incineration in the Netherlands; in Ecoinvent 3, 65\% of $\mathrm{CO}_{2}$ emission from the incineration of solid waste is biogenic, and the rest (35\%) is non-biogenic by default. Considering the waste generated during the chain elongation process mostly resulted from domestic kitchen waste, gardening waste and supermarket food waste, $\mathrm{CO}_{2}$ emissions from the incineration of solid waste has been modified into $100 \%$ biogenic. Table S2.3 presents the overview of the comparison between the assessed cases, i.e. Case LO, Case LS and Case PS. Table S2.4 shows the cases and parameters used for sensitivity analysis.

\section{Notes for internal reports (IR)}

Data and information from four internal reports (IR) were used for supporting this LCA work, as mentioned in the following tables. These IRs are not included in this thesis book; to access these IRs, please contact the Sub-department of Environmental Technology (ETE), Wageningen University \& Research. The IRs used in this work are listed below with their corresponding nmebers: 
IR1- 2014- Michael- Chain elongation of propionic acid, MSc thesis;

IR2- 2015- De Jong- Granulation in chain elongation, MSc thesis;

IR3- 2010- kuiper- MCFA recovery from fermentation broth, MSc thesis;

IR4- 2014- Vermeer- Selective Extraction of MCFA, MSc thesis. 
Table S2.1. Overview of the parameters used in each process of the assessed system, i.e. gate-toproduct life cycle of caproic acid production from organic waste via chain elongation.

\begin{tabular}{|c|c|c|}
\hline Process & Process parameters & Source \\
\hline \multirow{4}{*}{$\begin{array}{l}\text { Biological } \\
\text { Acidificatio } \\
\mathrm{n}(\mathrm{BAc})\end{array}$} & Hydraulic Retention Time $($ HRT $)=7$ days $^{\mathrm{a}}$ & \multirow{2}{*}{$\begin{array}{l}{ }^{39} \text { for organic fraction of } \\
\text { municipal solid waste } \\
(\text { OFMSW })\end{array}$} \\
\hline & $\begin{array}{l}\text { Dilution factor }=0.6 \mathrm{~L} \text { water } / \mathrm{kg} \text { OFMSW or } 0.8 \mathrm{~L} \\
\text { water } / \mathrm{kg} \mathrm{SFW}^{\mathrm{b}} \text {. }\end{array}$ & \\
\hline & Yield $(\mathrm{OFMSW})^{\mathrm{c}}=0.04 \mathrm{gCOD}_{\mathrm{SCFA}} / \mathrm{gCOD}_{\text {Waste }}$ & \multirow{2}{*}{$\begin{array}{l}\text { Internal experiment data } \\
\text { for supermarket food } \\
\text { waste (SFW); see } \\
\text { internal report } 1 \text { (IR1) } \\
\text { and IR2 attached in this } \\
\text { submission. }\end{array}$} \\
\hline & Yield $(\mathrm{SFW})^{\mathrm{c}}=0.2 \mathrm{gCOD}_{\mathrm{SCFA}} / \mathrm{gCOD}_{\text {waste }}$ & \\
\hline \multirow{5}{*}{$\begin{array}{l}\text { Chain } \\
\text { Elongation } \\
\text { (CE) }\end{array}$} & HRT $=11$ hours & \multirow{5}{*}{$\begin{array}{l}{ }^{39} \text { for OFMSW } \\
\text { Internal experiment data } \\
\text { for SFW; see IR } 1 \text { and } \\
\text { IR2. }\end{array}$} \\
\hline & $\begin{array}{l}\text { Yield }(\text { OFMSW })^{\mathrm{d}}= \\
\mathrm{gCOD}_{\text {Caproate }} /\left(\mathrm{gCOD}_{\mathrm{SCFA}}+\mathrm{gCOD}_{\text {Ethanol }}\right)\end{array}$ & \\
\hline & $\begin{array}{l}\text { Yield }(\mathrm{SFW})^{\mathrm{d}}= \\
\mathrm{gCOD}_{\text {Caproate }} /\left(\mathrm{gCOD}_{\mathrm{SCFA}}+\mathrm{gCOD}_{\text {Ethanol }}\right)\end{array}$ & \\
\hline & Require $\mathrm{CO}_{2}{ }^{\mathrm{e}}=4.60 \mathrm{ml}$ per g $\mathrm{COD}_{\text {Caproate }}$ & \\
\hline & Require $\mathrm{NaOH}^{\mathrm{f}}=0.36 \mathrm{~g}$ per g $\mathrm{COD}_{\text {Caproate }}$ & \\
\hline $\begin{array}{l}\text { Liquid- } \\
\text { Solid } \\
\text { Separation } \\
\text { (LSS) }\end{array}$ & Total solids $=5 \mathrm{wt} \%$ of CE effluent, disposed. & Assumption. \\
\hline $\begin{array}{l}\text { Chemical } \\
\text { Acidificatio } \\
\text { n (CAc) }\end{array}$ & $\begin{array}{l}\mathrm{HCl} \text { addition }^{\mathrm{g}}=0.75 \mathrm{ml} \mathrm{HCl}(37 \% ; 12 \mathrm{M}) \text { per } \mathrm{g} \\
\mathrm{COD}_{\text {Caproate }}\end{array}$ & $\begin{array}{l}\text { Based on calculation and } \\
\text { assumption. }\end{array}$ \\
\hline \multirow[t]{3}{*}{$\begin{array}{l}\text { Liquid- } \\
\text { Liquid } \\
\text { Extraction } \\
\text { (LLEx) }\end{array}$} & $\begin{array}{l}\text { The caproic acid extraction efficiency is defined as the } \\
\text { ratio between the amount of caproic acid entering the } \\
\text { solvent phase and the amount of caproic acid available } \\
\text { in the aqueous phase before extraction, i.e. the } \\
\text { fermentation broth, this is described as the formula } \\
\text { below: }\end{array}$ & \multirow[t]{3}{*}{$\begin{array}{l}\text { Steinbusch et al. } 2010^{22} \\
\text { Internal experimental } \\
\text { data (IR3 and IR4) }\end{array}$} \\
\hline & Extraction efficiency $=\mathrm{HC}_{\mathrm{Sol}, \mathrm{f}} / \mathrm{HC}_{\mathrm{ag}, \mathrm{i}}$ & \\
\hline & $\begin{array}{l}\text { where } \mathrm{HC}=\text { the amount of caproic acid, Sol= solvent } \\
\text { phase, } a q=\text { aqueous phase, } \mathrm{f}=\text { final (after extraction), } \mathrm{i}\end{array}$ & \\
\hline
\end{tabular}


= initial (before extraction).

The amount of solvent $\left(\mathrm{V}_{\mathrm{sol}}\right)$ required for reaching a specific caproate extraction efficiency ${ }^{\text {h }}$ can be calculated as described below:

$[\mathrm{HC}]_{\mathrm{Sol}}=\mathrm{D} *[\mathrm{HC}]_{\mathrm{aq}}$,

where $[\mathrm{HC}]=$ Concentration of caproic acid, D = the distribution coefficient of a compound between two liquid phases. Assuming that no chemical reaction occurs during the extraction, and the total amount of caproic acid is a constant, the formula above can be further expanded as below:

$\mathrm{HC}_{\text {sol.f }} / \mathrm{V}_{\mathrm{sol}}=\mathrm{D} * \mathrm{HC}_{\mathrm{aq}, \mathrm{f}} / \mathrm{V}_{\mathrm{aq}}=\mathrm{D} *\left(\mathrm{HC}_{\mathrm{aq}, \mathrm{i}}-\mathrm{HC}_{\mathrm{sol}, \mathrm{f}}\right) / \mathrm{V}_{\mathrm{aq}}$

By reorganising this formula, the extraction efficiency can be expressed as below:

Extraction efficiency $=\mathrm{HC}_{\mathrm{Sol}, \mathrm{f}} / \mathrm{HC}_{\mathrm{ag}, \mathrm{i}}=1 /$ $\left(1+\mathrm{V}_{\mathrm{aq}} / \mathrm{V}_{\mathrm{sol}} / \mathrm{D}\right)$

As Vaq and D are known, the amount of solvent required for achieving a specific extraction efficiency can be calculated. Subsequently, the amount of caproic acid extracted into the solvent can aslo be calculated.

The diffusion coefficient of caproate between acidified $\mathrm{CE}$ broth $(\mathrm{pH}=5)$ and ethyl caproate is around 22, based on the previous study ${ }^{22}$.

Lab-scale process: $95 \mathrm{wt} \%$ solvent is reused; $5 \mathrm{wt} \%$ Assumption. solvent is lost during extraction.

Pilot-scale process: $99.5 \mathrm{wt} \%$ solvent is reused; 0.5 $\mathrm{wt} \%$ solvent is lost during extraction.

Calculated based on the solubility of ethyl caproate (i.e. $0.629 \quad \mathrm{~g} / \mathrm{L}$; http://www.ymdb.ca/compounds/YMDB01381, accessed on 22nd Sep 2016). In the pilot-scale system, all ethyl caproate is assumed to be recovered, except for those dissolved, which is approximately $0.5 \mathrm{v} / \mathrm{v} \%$ of the total solvent added. In the lab-scale system, the solvent loss is assumed to be 10 times higher than in the pilot-scale system, which is about $5 \mathrm{v} / \mathrm{v} \%$ of the total solvent used.

Distillation Assume the caproic acid in the solvent phase was fully Assumption (Ds) recovered.

Lab-scale process: $95 \mathrm{wt} \%$ solvent is reused; $5 \mathrm{wt} \%$ Assumption 
solvent is lost during distillation.

Pilot-scale process: $99.5 \mathrm{wt} \%$ solvent is reused; 0.5 $\mathrm{wt} \%$ solvent is lost during distillation.

a. Longer HRT (21 days) was tried and resulted in 3 times higher VFA concentrations ${ }^{39}$. However, considering the process feasibility, an HRT of 7 days is used in this LCA.

b. Dilution factors taken from the literature ${ }^{39}$ and internal experiments. The former used dry anaerobic digestion and the latter used wet anaerobic digestion. Moreover, the solid contents of OFMSW and SFW were different. The amounts of water added for dilution is therefore subject to the feedstock used.

c. SCFAs include mainly acetate and butyrate. The yield was calculated from the experimental data from the literature ${ }^{39}$ and from the internal experiment.

d. $\mathrm{COD}_{\text {Ethanol }}=[$ Ethanol $](19.3 \mathrm{~g} / \mathrm{L}) * 2.1 \mathrm{gCOD} / \mathrm{gEthanol}=40.5 \mathrm{gCOD} / \mathrm{gEthanol} . \mathrm{L} . \mathrm{COD}_{\text {Caproate }}$ $=[$ Caproate $](12.6 \mathrm{~g} / \mathrm{L}) * 1.9 \mathrm{gCOD} / \mathrm{gCaproate}=23.9 \mathrm{gCOD} / \mathrm{gCaproate} . \mathrm{L}$.

e. $240 \mathrm{ml}$ gaseous $\mathrm{CO}_{2}$ was fed into the chain elongation bioreactor every day. Regarding the HRT for the chain elongation bioreactor (11 hours), around 52.23 gCOD caproate was produced per day ${ }^{39}$.

f. Based on the metabolism, 2 moles of protons result from the production of $220.4 \mathrm{gCOD}$ caproate $(1 \mathrm{~mole})^{26,41}$. $\mathrm{NaOH}$, which is required to ensure $\mathrm{pH}$ stability of the whole reactor, is not included as we assume the reactor has certain $\mathrm{pH}$-buffer capacity.

g. 1 mole of caproate requires 1 mole of $\mathrm{HCl}$ to be acidified into caproic acid, which means 4.5 $\mathrm{ml} \mathrm{HCl}(1 \mathrm{M})$ per $\mathrm{g} \mathrm{COD}_{\text {Caproate }}$ is required for CAc. This number is multiplied by 2 to give sufficient protons to lower the $\mathrm{pH}$ of the solution (i.e. for other MCFAs but preferably not SCFAs) and to overcome the buffer capacity of the solution.

h. Extraction efficiency is limited by the distribution coefficient of the solvent used (concentration in solvent phase v.s. concentration in aqueous phase). For ethyl caproate, this is assumed to be 22 when $\mathrm{pH}$ of the solution is around $4.9^{22}$. 
Table S2.2. The overview of the activity; material and energy used, as well as their purpose of use and data sources for calculating the LCIA in this study.

\begin{tabular}{lll}
\hline $\begin{array}{l}\text { Activity/Material Unit } \\
\text { /Energy }\end{array}$ & Purpose of use \\
& \\
\hline $\begin{array}{l}\text { Solid waste } \\
\text { management }\end{array}$ & & $\begin{array}{l}\text { Treat the solid residue from } \\
\text { BAc, CE, LSS \& Ds in Case } \\
\end{array}$ \\
& & LO and LS.
\end{tabular}

Comment/Data source (in Ecoinvent 3 or otherwise specified)

Municipal solid waste $\{\mathrm{NL}\} \mid$ treatment of, incineration | Alloc Def, $\mathrm{U}$. The biogenic $\mathrm{CO}_{2}$ content in the total $\mathrm{CO}_{2}$ emission is set as $100 \%$ instead of $65 \%$ (the default value in Ecoinvent 3)

\begin{tabular}{|c|c|c|c|}
\hline & per $\mathrm{kg}$ & $\begin{array}{l}\text { Treat the solid residue from } \\
\text { BAc, CE, LSS \& Ds in Case } \\
\text { PS, as ChainCraft advised. }\end{array}$ & $\begin{array}{l}\text { Biowaste }\{\text { RoW }\} \mid \text { market for } \mid \\
\text { Alloc Def, } U\end{array}$ \\
\hline $\begin{array}{l}\text { Wastewater } \\
\text { treatment }\end{array}$ & per $\mathrm{L}$ & $\begin{array}{l}\text { Treat the wastewater from } \\
\text { LLEx \& Ds. }\end{array}$ & $\begin{array}{l}\text { Wastewater, from residence } \\
\{\text { RoW }\} \mid \text { treatment of, capacity } \\
1.1 \mathrm{E} 101 / \text { year } \mid \text { Alloc Def, U }\end{array}$ \\
\hline $\begin{array}{l}\text { Heat from } \\
\text { natural gas }\end{array}$ & per MJ & Heat up bioreactors. & $\begin{array}{l}\text { Heat, in chemical industry } \\
\{\text { RER }\} \mid \text { market for } \mid \text { Alloc Def, } U\end{array}$ \\
\hline Electricity & per MJ & $\begin{array}{l}\text { For operation of apparatus and } \\
\text { bioreactors. }\end{array}$ & $\begin{array}{l}\text { Electricity, low voltage }\{\mathrm{NL}\} \mid \\
\text { market for } \mid \text { Alloc Def, } \mathrm{U}\end{array}$ \\
\hline \multirow[t]{3}{*}{ Ethyl caproate } & per $\mathrm{kg}$ & $\begin{array}{l}\text { Extraction solvent in LLEx in } \\
\text { all baseline cases. }\end{array}$ & $\begin{array}{l}\text { Ethyl acetate }\{\mathrm{GLO}\} \mid \text { market for } \mid \\
\text { Alloc Def, } U\end{array}$ \\
\hline & & & $\begin{array}{l}\text { Use the data for ethyl acetate as a } \\
\text { simulation due to the lack of data } \\
\text { for ethyl caproate. }\end{array}$ \\
\hline & per kg & For sensitivity analysis. & White mineral oil, at plant/RNA \\
\hline $\begin{array}{l}\text { Hydrochloric } \\
\text { acid }\end{array}$ & per $\mathrm{kg}$ & $\begin{array}{l}\text { Protonation of caproate into } \\
\text { carpoic acid. }\end{array}$ & $\begin{array}{l}\text { Hydrochloric acid, without water, } \\
\text { in } 30 \% \text { solution state }\{\text { RER }\} \mid \\
\text { market for } \mid \text { Alloc Def, } U\end{array}$ \\
\hline $\begin{array}{l}\text { Carbon dioxide } \\
\text { (Liquefied) }\end{array}$ & per $\mathrm{kg}$ & $\begin{array}{l}\text { Essential substrate for } \\
\text { supporting microbial growth } \\
\text { in CE. }\end{array}$ & $\begin{array}{l}\text { Carbon dioxide, liquid }\{\text { RER }\} \mid \\
\text { market for } \mid \text { Alloc Def, } U\end{array}$ \\
\hline $\begin{array}{l}\text { Sodium } \\
\text { hydroxide } \\
(\mathrm{NaOH})\end{array}$ & per $\mathrm{kg}$ & $\begin{array}{l}\text { Base for neutralisation of } \mathrm{pH} \\
\text { in BAc and CE. }\end{array}$ & $\begin{array}{l}\text { Neutralising agent, sodium } \\
\text { hydroxide-equivalent }\{\mathrm{GLO}\} \mid \\
\text { market for } \mid \text { Alloc Def, } U\end{array}$ \\
\hline
\end{tabular}




\begin{tabular}{|c|c|c|c|}
\hline & per kg & $\begin{array}{l}\text { A more recent LCA study on } \\
\mathrm{NaOH}(2013) \text { for sensitivity } \\
\text { analysis. The data from } \\
\text { Ecoinvent } 3 \text { was published in } \\
2007 \text {, with the process data } \\
\text { collection occurring before } \\
2007 \text {. }\end{array}$ & Literature $^{102}$ \\
\hline \multirow[t]{4}{*}{ Ethanol } & per kg & $\begin{array}{l}\text { Essential substrate for CE in } \\
\text { all baseline cases. }\end{array}$ & $\begin{array}{l}\text { Ethanol, without water, in } 95 \% \\
\text { solution state, from fermentation } \\
\{\text { RoW }\} \mid \text { ethanol production from } \\
\text { maize } \mid \text { Alloc Def, } U\end{array}$ \\
\hline & per kg & $\begin{array}{l}\text { Sugarcane bioethanol for } \\
\text { sensitivity analysis. }\end{array}$ & $\begin{array}{l}\text { Ethanol, without water, in } 95 \% \\
\text { solution state, from fermentation } \\
\{B R\} \mid \text { ethanol production from } \\
\text { sugar cane } \mid \text { Alloc Def, } U\end{array}$ \\
\hline & per kg & $\begin{array}{l}\text { Fossil-based ethanol for } \\
\text { sensitivity analysis. }\end{array}$ & $\begin{array}{l}\text { Ethanol, without water, in } 99.7 \% \\
\text { solution state, from ethylene } \\
\{\text { RER } \mid \text { ethylene hydration | } \\
\text { Alloc Def, U }\end{array}$ \\
\hline & per kg & For sensitivity analysis. & $\begin{array}{l}\text { Ethanol, without water, in } 95 \% \\
\text { solution state, from fermentation } \\
\{\mathrm{CH}\} \mid \text { ethanol production from } \\
\text { grass } \mid \text { Alloc Def, } U\end{array}$ \\
\hline Water & per kg & Dilution. & $\begin{array}{l}\text { Water, deionised, from tap water, } \\
\text { at user }\{\mathrm{GLO}\} \mid \text { market for } \mid \text { Alloc } \\
\text { Def, } U\end{array}$ \\
\hline
\end{tabular}


Table S2.3. The setup of the three cases assessed in the present study. L refers to lab-scale and $\mathrm{P}$ refers to pilot-scale. O refers to organic fraction of municipal solid waste (OFMSW), and $\mathrm{S}$ refers to supermarket food waste (SFW).

\begin{tabular}{|c|c|c|c|}
\hline & Case LO & Case LS & Case PS \\
\hline Feedstock & $\begin{array}{l}\text { OFMSW, } 90 \% \\
\text { gardening waste }+ \\
10 \% \text { kitchen food } \\
\text { waste }\end{array}$ & $\begin{array}{l}\text { SFW, } 100 \% \text { food } \\
\text { waste from } \\
\text { supermarket food } \\
\text { processing chain }\end{array}$ & $\begin{array}{l}\text { SFW, } 100 \% \text { food } \\
\text { waste from } \\
\text { supermarket food } \\
\text { processing chain }\end{array}$ \\
\hline BAc process & $\begin{array}{l}\text { Dry anaerobic } \\
\text { digestion }\end{array}$ & $\begin{array}{l}\text { Wet anaerobic } \\
\text { digestion }\end{array}$ & $\begin{array}{l}\text { Wet anaerobic } \\
\text { digestion }\end{array}$ \\
\hline Solvent recovery efficiency & $90 \%$ & $90 \%$ & $99 \%$ \\
\hline Solid waste management & $\begin{array}{l}\text { Incineration with } \\
\text { heat recovery }\end{array}$ & $\begin{array}{l}\text { Incineration with } \\
\text { heat recovery }\end{array}$ & $\begin{array}{l}\text { Anaerobic } \\
\text { digestion with } \\
\text { energy recovery }\end{array}$ \\
\hline
\end{tabular}


Table S2.4. Overview of the cases used in the sensitivity analysis of this study and the description of the parameters used.

\begin{tabular}{|c|c|c|}
\hline Cases/parameters & Description & Reference \\
\hline Baseline case & $\begin{array}{l}\text { The life-cycle impacts of the caproic acid } \\
\text { production from organic waste in Case } \\
\text { LO, LS and PS assessed in this study. }\end{array}$ & This study \\
\hline $\begin{array}{l}\text { Use of sugarcane } \\
\text { bioethanol, Brazil }\end{array}$ & Alternative source for bioethanol & $\begin{array}{l}\text { Ecoinvent } 3 \text { (Ethanol, } \\
\text { without water, in } 95 \% \\
\text { solution state, from } \\
\text { fermentation }\{\mathrm{BR}\} \mid \text { ethanol } \\
\text { production from sugar cane } \\
\mid \text { Alloc Def, } U \text { ) }\end{array}$ \\
\hline $\begin{array}{l}\text { Use of fossil-based } \\
\text { ethanol, Europe }^{\dagger}\end{array}$ & Alternative source for bioethanol & $\begin{array}{l}\text { Ecoinvent } 3 \text { (Ethanol, } \\
\text { without water, in } 99.7 \% \\
\text { solution state, from } \\
\text { ethylene }\{\text { RER }\} \mid \text { ethylene } \\
\text { hydration | Alloc Def, U) }\end{array}$ \\
\hline $\begin{array}{l}\text { Use of } \\
\text { lignocellulosic } \\
\text { bioethanol (from } \\
\text { grass), Europe }\end{array}$ & Alternative source for bioethanol & $\begin{array}{l}\text { Ecoinvent } 3 \text { (Ethanol, } \\
\text { without water, in } 95 \% \\
\text { solution state, from } \\
\text { fermentation }\{\mathrm{CH}\} \mid \text { ethanol } \\
\text { production from grass } \mid \\
\text { Alloc Def, } \mathrm{U} \text { ) }\end{array}$ \\
\hline $\begin{array}{l}\text { Precise control of } \\
\text { ethanol dose during } \\
\text { CE }(50 \% \text { ethanol } \\
\text { dose reduction })\end{array}$ & $\begin{array}{l}\text { Assumption of the minimal ethanol } \\
\text { required to maintain the same caproate } \\
\text { production rate as in the baseline } \\
\text { scenarios. The assumption is based on the } \\
\text { microbial reaction stoichiometry of } \\
\text { Clostridium kluyveri converting acetate } \\
\text { and ethanol to caproate. }\end{array}$ & Seedorf et al. $2008^{41}$ \\
\hline $\begin{array}{l}\text { Mineral oil as the } \\
\text { extraction solvent }\end{array}$ & $\begin{array}{l}\text { Mineral oil was used in the internal } \\
\text { experiments as an extraction solvent for } \\
\text { caproic acid, which has a similar } \\
\text { extraction performance to ethyl caproate. } \\
\text { The LCI data was derived from } \\
\text { Ecoinvent } 3 \text {. }\end{array}$ & $\begin{array}{l}\text { Internal experiments (IR3 } \\
\text { and IR4). } \\
\text { Ecoinvent } 3 \text { ( White } \\
\text { mineral oil, at plant/RNA) }\end{array}$ \\
\hline $\begin{array}{l}\mathrm{NaOH} \text { alternative } \\
\text { data }\end{array}$ & $\begin{array}{l}\text { The use of a more recent LCI data set to } \\
\text { assess the possible reduction on life-cycle } \\
\text { impact of } \mathrm{NaOH} \text { with the improved } \\
\text { production process. }\end{array}$ & Thannimalay et al. $2013^{102}$ \\
\hline
\end{tabular}


${ }^{\dagger}$ Excluding end-of-life emissions (e.g. $\mathrm{CO}_{2}$ during combustion), which are usually for fossilbased ethanol and are considerably higher than for bio-ethanol, depending on the applications. 


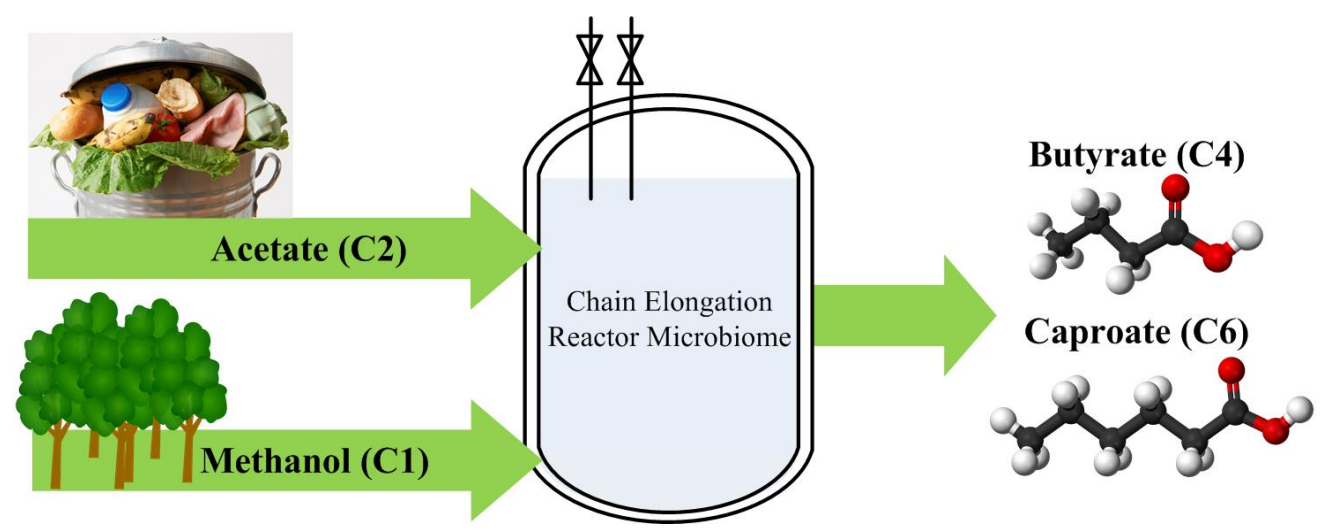




\title{
Chapter 3. Methanol chain elongation for butyrate and caproate production
}

\begin{abstract}
Microbial chain elongation is an emerging mixed culture biotechnology converting acetate into valuable biochemicals by using ethanol as an external electron donor. In this study we proposed to test another potential electron donor, methanol, in microbial chain elongation. Methanol can be produced through the thermochemical conversion of lignocellulosic biowaste. Use of methanol integrates lignocellulosic feedstocks and thermochemical conversion technologies into microbial chain elongation. A proof-of-principle study of microbial chain elongation using methanol and acetate was performed in both a batch and a continuous experiment. In the batch experiment, butyrate $(4.2 \mathrm{~g} / \mathrm{L})$ and caproate $(0.1 \mathrm{~g} / \mathrm{L})$ production from methanol and acetate was observed. A mixed culture microbiome taken from a previous chain elongation reactor fed with ethanol was responsible for the observed organic acid production. The continuous experiment was performed in an upflow anaerobic bioreactor (UAB). The hydraulic retention time (HRT) was 36 hours and the operational period lasted for 45 days. In the continuous experiment, butyrate production (Rate> 1.5 g/L.day) was observed; the caproate concentration was below the detection limit during the entire continuous operational period. In both experiments, methanol and acetate were both substrates contributing to the butyrate production. To the authors' current knowledge, this study is the first attempt at a mixed culture fermentation utilising methanol and acetate for biochemical production. Further research should focus on elevating the butyrate production rate and concentration in the continuous operation of methanol chain elongation, which may stimulate caproate formation.
\end{abstract}

A modified version of this chapter was published as: Chen, W. S.; Ye, Y.; Steinbusch, K. J. J.; Strik, D. P. B. T. B.; Buisman, C. J. N. Methanol as an alternative electron donor in chain elongation for butyrate and caproate formation. Biomass Bioenergy. 2016, 93, 201-208. 


\subsection{Introduction}

Pollution introduced by combustion of fossil fuels has triggered the shift towards using cleaner and more renewable alternative feedstocks for chemical and fuel production. Organic waste is a potential carbon resource for chemical and fuel production. Organic waste is nonfossil based, abundantly available and does not compete with food production. Utilising organic waste to produce biochemicals and biofuels can offer a win-win solution. The carbon resource in organic waste is recovered while a contribution to the demand for renewable chemicals and fuels can be met.

Microbial chain elongation (hereinafter referred to as chain elongation) is a novel mixed culture biotechnology converting organic waste into precursors of biofuels and biochemicals ${ }^{36,37}$. Chain elongation employs a mixed culture microbiome that converts small molecules derived from organic waste (e.g. acetate, propionate, $\mathrm{CO}_{2}$ and ethanol) into a group of valuable biochemicals, the so-called "medium chain fatty acids" (MCFAs, saturated fatty acids containing 6-12 carbons; e.g. caproate, heptanoate and caprylate) ${ }^{32,}, 36,37$. Compared with pure culture biotechnologies, advantages of mixed culture biotechnologies include: no sterilization requirement, the adaptive capacity to changing conditions owing to microbial diversity and the capacity to use mixed substrates ${ }^{20}$. The product of chain elongation, MCFAs, can be used as a commodity chemical ${ }^{67,68}$ or serve as a precursor of various biofuels and biochemicals ${ }^{26,65,106}$. Current production of MCFAs relies on plant oils like coconut and palm kernel oils ${ }^{107}$, both of which are often produced on environmentally undesired plantations. Such intensive agriculture has many potential environmental consequences $^{13}$ including Greenhouse Gas (GHG) emission ${ }^{9}$, biodiversity $\operatorname{loss}^{108}$ and competition for arable land with food production ${ }^{11,56}$. Chain elongation offers a process that can produce MCFAs with a reduced land footprint depending on its feedstocks. Acetate and $\mathrm{CO}_{2}$ are two essential substrates used in chain elongation. Both acetate and $\mathrm{CO}_{2}$ can be 
abundantly produced from various organic waste feedstocks through a biochemical conversion process like the acidification but without land use requirement ${ }^{26}$.

Ethanol is also an essential substrate for chain elongation. Currently to carry out chain elongation either an ethanol-containing waste stream (e.g. corn fermentation beer) is used as the feedstock ${ }^{37}$, or addition of ethanol during the fermentation is required ${ }^{38}$. Ethanol contributes to at least two-third of carbon in the end product of chain elongation, as for example in caproate ${ }^{26,41}$. Currently ethanol is produced mainly from crops like sugarcane and corn ${ }^{109}$. These crops require arable land for their production and, in most cases, are more costly compared with an organic waste feedstock. Reducing or replacing the use of cropbased ethanol in chain elongation is of importance in order to further improve the environmental performance and cost-effectiveness of chain elongation.

Lignocellulosic biowaste is one of the potential organic waste streams that can be used to reduce or replace the crop-based ethanol use in chain elongation. For example, lignocellulosic bioethanol can be produced by employing enzymatic hydrolysis and fermentation. This process is currently under development and it may be commercially available to replace the sugarcane- and corn-based bioethanol in the near future ${ }^{50}$. Vasudevan et al. (2014) demonstrated another possible use of lignocellulosic biowaste in chain elongation. The synthesis gas produced through thermochemical processing of the lignocellulosic biowaste was converted into bioethanol through a pure culture fermentation process. The bioethanol produced from the synthetic gas was then used as the feedstock for chain elongation ${ }^{40}$.

In this study we investigated another strategy for using lignocellulosic biowaste in chain elongation: the use of methanol as an alternative electron donor in chain elongation. Methanol can be produced from synthesis gas or several other waste streams through chemical processes ${ }^{49}$. Moreover, the production of lignocellulosic methanol has been commercialised and implemented ${ }^{50}$. The use of methanol in chain elongation can expand the 


\section{Chapter 3}

feedstock range of chain elongation and increase the resource security for the production of MCFAs.

To use methanol for the biological formation of MCFAs was attempted in four previous studies using monocultures. Keneally and Waselefsky (1985) blended methanol into the growth medium of a pure culture Clostridium kluyveri, a known bacterium elongating short chain fatty acids (SCFAs; saturated fatty acid containing less than 6 carbons) and ethanol into MCFAs. It was reported, though, that methanol was not metabolised by C.kluyveri. Another microorganism, Eubacterium limosum, was on the other hand reported to produce small amounts of caproate from methanol and SCFAs in a pure culture incubation ${ }^{47,55,110}$. Genthner et al. $(1981)$ reported the production of butyrate $(35.68 \mathrm{mMC} ; \mathrm{mMC}=$ millimolar carbon $)$ and caproate $(0.78 \mathrm{mMC})$ from a pure culture E.limosum growing on methanol (50 $\mathrm{mMC})$ and acetate $(60 \mathrm{mMC})^{55}$. Lindley et al. (1987) also reported the caproate production with a pure culture E.limosum growing on methanol (100 mMC), $\mathrm{CO}_{2}$ and butyrate (400-1600 mMC) with a yeast extract supplement $(0.5 \mathrm{~g} / \mathrm{L})^{47}$. Tarasov et al. (2011) lately reported caproate production from methanol and $\mathrm{CO}_{2}$ by a pure culture growth of E.limosum but the actual data were not given ${ }^{110}$. So far a mixed culture fermentation converting methanol and SCFAs into MCFAs has not been reported. Nevertheless, the existence of these pure culture studies implies the potential feasibility of such fermentation process.

This study investigated the feasibility of using methanol and acetate as the substrates for chain elongation (Hereinafter referred to as "methanol chain elongation") to produce butyrate and caproate. These are typical chemicals produced by chain elongation using ethanol and acetate as the substrates (Hereinafter referred to as "ethanol chain elongation"). Both batch and continuous methanol chain elongation experiments were performed. The batch test aimed at demonstrating the proof-of-principle of methanol chain elongation. Several combinations of substrates and inoculum were examined in the batch experiments. Following the batch 
experiments, an upflow anaerobic bioreactor (UAB) was set up to demonstrate the feasibility of the continuous methanol chain elongation.

\subsection{Methodology}

\subsubsection{Batch experiment}

Two batch experiments were carried out in this study, the $1^{\text {st }}$ batch experiment and the $2^{\text {nd }}$ batch experiment. The code and experimental conditions of both the $1^{\text {st }}$ and $2^{\text {nd }}$ batch experiments can be found in Table 3.1. The $1^{\text {st }}$ batch experiment tested a set of various combinations of the substrates and inoculum. Two types of inoculum, the mixed culture inoculum with $(+\mathrm{E})$ or without pure culture Eubacterium limosum (ATCC 8486) addition, were used in the $1^{\text {st }}$ batch experiment. The mixed culture inoculum was taken from the fermentation broth of a UAB that was used for performing ethanol chain elongation ${ }^{35,36}$. The E.limosum added was incubated in a pure culture batch prior to the inoculation to ensure its activity, as further described in the Support Information for Chapter 3 (SI3). The effect of the addition of methanol (Me) and acetate (Ac) were also examined in the $1^{\text {st }}$ batch experiment. Two blanks $(\mathrm{B}$ and $\mathrm{B}+\mathrm{E})$ without methanol and acetate addition were used to estimate the contribution of the yeast extract to the organic acid formed during the batch experiments. Yeast extract is a commonly used substance in chain elongation for supporting the microbial growth $^{32,36,47,88,111-113}$.

The $2^{\text {nd }}$ batch experiment was carried out to enrich the biomass for later use, i.e. the inoculum for the continuous methanol chain elongation experiment. For the $2^{\text {nd }}$ batch experiment, the mixed culture fermentation broth from the MeAc+E was used as the sole inoculum. $\mathrm{MeAc}+\mathrm{E}$ had the highest butyrate production from methanol and acetate in the $1^{\text {st }}$ batch; therefore, it was used as the inoculum for further research. The procedure for preparing the batch test and the inoculum are documented in the SI3. All batch experiments in the present study were triplicated. 


\section{Chapter 3}

Table 3.1. Overview of the substrate, the headspace composition and the inoculum used in both $1^{\text {st }}$ and $2^{\text {nd }}$ batch experiments.

\begin{tabular}{|c|c|c|c|c|c|c|c|}
\hline \multirow{2}{*}{ Batch Code } & \multicolumn{6}{|c|}{$1^{\text {st }}$ batch } & \multirow{2}{*}{$\begin{array}{l}2^{\text {nd }} \text { batch } \\
2-\mathrm{MeAc}+\mathrm{E}\end{array}$} \\
\hline & B & $\mathrm{B}+\mathrm{E}$ & $\mathrm{Me}$ & $\mathrm{Me}+\mathrm{E}$ & $\mathrm{MeAc}$ & $\mathrm{MeAc}+\mathrm{E}$ & \\
\hline E.limosum & - & + & - & + & - & + & $-*$ \\
\hline Methanol (mM) & - & - & 100 & 100 & 100 & 100 & $100 / 200^{\dagger}$ \\
\hline Acetate (mM) & - & - & - & - & 50 & 50 & 50 \\
\hline $\begin{array}{l}\text { Headspace } \\
\text { (1.5 bar) }\end{array}$ & \multicolumn{7}{|c|}{$80 \% \mathrm{~N}_{2}+20 \% \mathrm{CO}_{2}$ for all batches } \\
\hline \multicolumn{8}{|c|}{$\begin{array}{l}\text { *Inoculum for } 2^{\text {nd }} \text { batch was taken from MeAc+E in the } 1 \text { st batch. } \\
+ \text { Initial methanol concentration was } 100 \mathrm{mM} \text {, and extra methanol }(+100 \mathrm{mM}) \text { was added } \\
\text { during the incubation to present the substrate depletion. }\end{array}$} \\
\hline
\end{tabular}

The composition of the growth medium was adapted from the previous ethanol chain elongation studies ${ }^{32,36}$. The medium contained $\mathrm{NH}_{4} \mathrm{H}_{2} \mathrm{PO}_{4}-3.6 \mathrm{~g} / \mathrm{L}, \mathrm{MgCL}_{2} \cdot 6 \mathrm{H}_{2} \mathrm{O}-0.33 \mathrm{~g} / \mathrm{L}$, $\mathrm{MgSO}_{4} .7 \mathrm{H}_{2} \mathrm{O}-0.2 \mathrm{~g} / \mathrm{L}, \mathrm{CaCl}_{2} .2 \mathrm{H}_{2} \mathrm{O}-0.2 \mathrm{~g} / \mathrm{L}, \mathrm{KCl}-0.15 \mathrm{~g} / \mathrm{L}$, yeast extract $-1 \mathrm{~g} / \mathrm{L}$, Vitamin B solution $1 \mathrm{ml} / \mathrm{L}$ and trace element solution $1 \mathrm{ml} / \mathrm{L}^{113}$. In addition to the growth medium, different methanol and acetate supplements were used in different batches as shown in Table 3.1. In the $1^{\text {st }}$ batch experiment, the effects of methanol $(100 \mathrm{mM})$ and acetate $(50 \mathrm{mM}$, in the form of sodium acetate) supplements on the mixed culture fermentation were studied. In the $2^{\text {nd }}$ batch experiment, methanol $(100 \mathrm{mM})$, acetate $(50 \mathrm{mM})$ and $\mathrm{CO}_{2}$ were all used as substrates. Moreover, the methanol concentration was later (at day7) elevated to $200 \mathrm{mM}$ to prevent the substrate depletion.

A gas exchanger was used to flush the headspace of all the batches. The headspace was first vacuumed and subsequently filled with pure nitrogen gas up to 1.5 bar. This procedure was repeated for 5 times, then the headspace was vacuumed again and filled with the desired headspace composition (a gas mixture containing $80 \% \mathrm{~N}_{2}$ and $20 \% \mathrm{CO}_{2}$ up to 1.5 bar). The 
gas exchanger may have trace amounts of impurities; moreover, not all the batch bottles were flushed at once as the gas exchanger had only nine outlets. Therefore, the headspace gas composition of all the batches were measured an hour after the gas exchange. Two impurities in gas phase were found in some batches at day $0: 1.6 \% \mathrm{CH}_{4}$ in $\mathrm{Me}$ and $\mathrm{MeAc} ; 2.8 \% \mathrm{H}_{2}$ in $\mathrm{Me}+\mathrm{E}$ and MeAc+E. For the other components $\left(\mathrm{O}_{2}, \mathrm{~N}_{2}\right.$ and $\left.\mathrm{CO}_{2}\right)$ the compositions were approximately the same in all the batches.

To estimate and further exclude the carbon contribution of the yeast extract to the product formation, a composition of yeast extract, $\mathrm{CH}_{1.7} \mathrm{O}_{0.5} \mathrm{~N}_{0.2}$, was derived from a previous study and used in this study to calculate the initially available carbon from the yeast extract $\operatorname{added}^{114}$. Based on this estimation and the concentration of the yeast extract used $(1 \mathrm{~g} / \mathrm{L})$, 34.4 8mMC was available assuming that the yeast extract added was completely metabolised.

\subsubsection{Continuous reactor}

An upflow anaerobic bioreactor (UAB, Figure 3.1) with 1-L capacity (including a 0.2-L headspace) was used to perform the continuous methanol chain elongation. The design of the UAB was identical to those used in the previous ethanol chain elongation studies except the biomass retention and the gas outflow quantification ${ }^{35,36,38,39}$. Previous ethanol chain elongation studies used polyurethane cubes for retaining the biomass in the continuous operation. In this study polyurethane cubes were not used. A gas counter (Ritter MGC-1, Germany) was installed in the UAB to quantify the gas production during the continuous operation of methanol chain elongation, which was not implemented in the previous ethanol chain elongation studies. The growth medium was the same as the batch experiment, except for the acetate and methanol concentrations as specified in Table 3.2. 

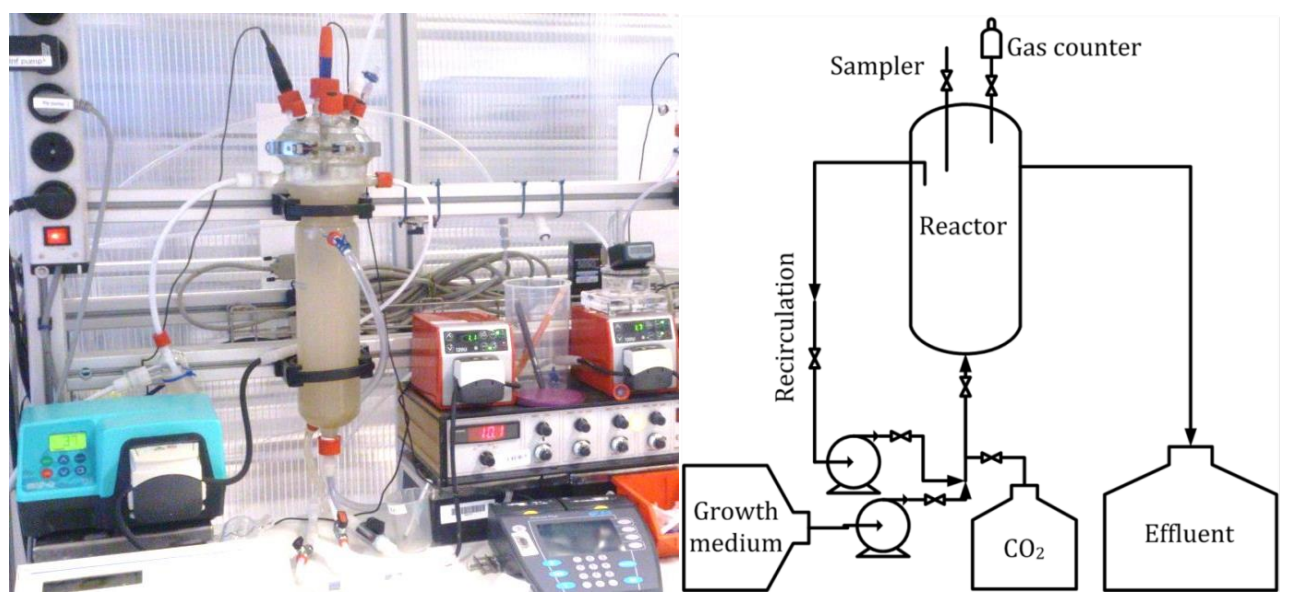

Figure 3.1. The setup of the upflow anaerobic bioreactor (UAB) used for performing the continuous methanol chain elongation in this study.

A continuous methanol chain elongation was performed with the UAB. The UAB was operated for 45 days and consisted of three phases: the start-up batch operation (Phase I), the continuous operation without $\mathrm{pH}$ control (Phase II) and the continuous operation with $\mathrm{pH}$ control at $6.5 \pm 0.1$ (Phase III). During the start-up phase, $200 \mathrm{mM}$ acetate and $300 \mathrm{mM}$ methanol were added to supply sufficient substrates for starting up the methanol chain elongation. The fermentation broth was internally recirculated $(150 \mathrm{ml} / \mathrm{minute})$ with a peristaltic pump (Watson Marlow 323, UK) during the whole incubation period. The batch operation (Phase I) lasted 13 days. After the batch operation (Phase I), the UAB was switched into a continuous operation and flushed with a nitrogen gas flow to ensure the anaerobic condition in the UAB. The growth medium was fed into the UAB with another peristaltic pump (Watson Marlow 120U, UK) to maintain the hydraulic retention time (HRT) at 36 hours. The $\mathrm{pH}$ of the fermentation broth was kept at $6.5 \pm 0.1$ by adjusting the amount of sodium hydroxide added in the growth medium. Gaseous $\mathrm{CO}_{2}$ was continuously supplied to the UAB; the supply rate was controlled with a mass flow controller (Brooks mass flow 
controller 5850E, USA) at $240 \mathrm{ml} \mathrm{CO}$ /day. A water bath (Julabo MA-4, Germany) was used to maintain the reactor temperature at $30^{\circ} \mathrm{C}$.

Table 3.2. The operational conditions in the different phases of the continuous methanol chain elongation UAB operation

\begin{tabular}{llll}
\hline Phase conditions & Phase I (Start-up) & Phase-II & Phase III \\
\hline Operational mode & Batch & Continuous & Continuous \\
Phase duration (days) & $0-13$ & $14-24$ & $25-45$ \\
pH & $6.0 \pm 0.1$ & $5.9 \pm 0.0$ & $6.5 \pm 0.1$ \\
& & & (Controlled) \\
Acetate (mM) & 100 & 100 & 100 \\
Methanol (mM) & 300 & 200 & 200 \\
\hline
\end{tabular}

\subsubsection{Analysis}

Liquid and gas samples were taken twice a week in all batch experiments in the present study. The pressure in the headspace was measured with a gas pressure meter (GMH3150, Greisinger Electronics, Germany) while taking the gas samples. All the batch experiments were triplicated. The samples from all the triplicated batches were taken and analysed. The mean values and the standard deviations were calculated and given in all the figures. In the continuous experiment, a liquid sample from the fermentation broth and a gas sample from the reactor headspace were taken 5 times and 2 times per week, respectively.

All liquid samples taken were analysed by a Gas Chromatography (GC; HP5890, USA) to determine the concentrations of both SCFAs and MCFAs (C2-C8) that may present in the fermentation broth, including all their isomers (except for the isomer of caprylate). The methanol and ethanol concentration were analysed by another GC (HP5890, USA). The gas sample taken from the headspace were analysed to identify the gas composition. Oxygen, nitrogen, methane and carbon dioxide were measured in one GC (Shimadzu GC-2010, Japan) 


\section{Chapter 3}

and hydrogen was measured separately in another GC (HP-5890, USA). The sample preparation and the GC programmes used were the same as those used by several previous ethanol chain elongation studies ${ }^{32,36,88}$. Together with the gas outflow quantified by the gas counter, the carbon outflow in the form of gaseous compound was determined (See also SI3 for the $\mathrm{CO}_{2}$ quantification).

\subsection{Results and discussion}

\subsubsection{Methanol chain elongation in the batch experiments}

\subsubsection{Chain elongation of methanol and acetate producing butyrate}

In the $1^{\text {st }}$ batch experiment butyrate was produced in the presence of methanol, acetate and $\mathrm{CO}_{2}$ regardless of the type of inoculum used (Figure 3.2; $48 \mathrm{mMC}$ butyrate in MeAc and 52 $\mathrm{mMC}$ in $\mathrm{MeAc}+\mathrm{E})$. In both $\mathrm{MeAc}$ and $\mathrm{MeAc}+\mathrm{E}$, the four potential substrates that might have contributed to the butyrate formation were methanol, acetate, $\mathrm{CO}_{2}$ and yeast extract. Based on the overall production and consumption shown in Figure 3.2, acetate (18 and $22 \mathrm{mMC})$ and methanol (36 and $37 \mathrm{mMC}$ ) were the main substrates contributing to the butyrate formation. $\mathrm{CO}_{2}$ did not likely contribute to the butyrate formation as there was not net $\mathrm{CO}_{2}$ consumption (See Figure S3.1 in the support information SI3). Yeast extract might contribute to the butyrate formation in MeAc and MeAc+E. According to the blanks (B \& B+E), 2.9 and 3.1mMC of butyrate were produced from the yeast extract. Assuming the conversion of the yeast extract added in MeAc and MeAc+E was similar to such in the blanks, it could be calculated that about $6 \%$ of the total butyrate production in both MeAc and MeAc+E was from the yeast extract. The remaining $94 \%$ butyrate production could be attributed to the consumption of methanol and acetate. In addition to butyrate, $\mathrm{CH}_{4}(0.2 \mathrm{mmole}$ in $\mathrm{MeAc}$ and 0.4 mmole in $\mathrm{MeAc}+\mathrm{E})$ and $\mathrm{CO}_{2}(0.4$ mmole MeAc and 0.4 mmole in MeAc+E) were also produced in MeAc and MeAc+E as shown in Figure S3.1. 


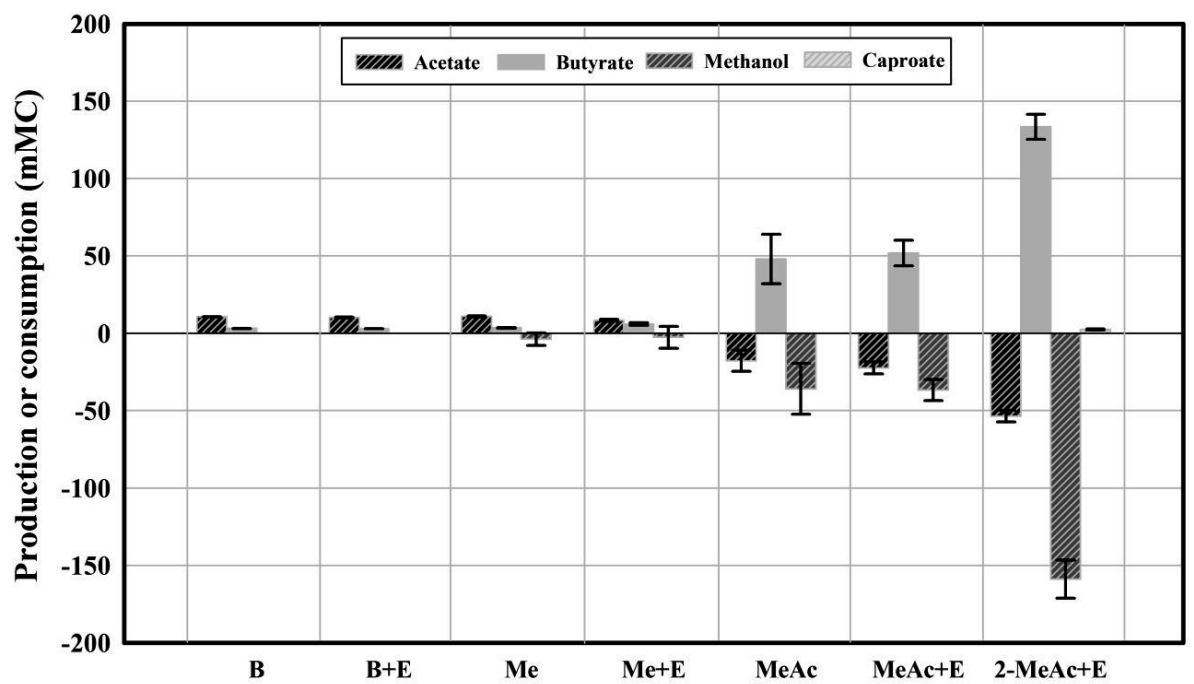

Figure 3.2. The production and consumption of the main substances in the $1^{\text {st }}$ (day 0 - day 21 ) and $2^{\text {nd }}$ (day 7 - day 17 ) batch experiments. For the meaning of the batch codes refers to Table 3.1. In 2-MeAc+E caproate was observed in small amount (refer to Figure 3.3), which was not shown here as one of the main substances. All experiments were triplicated, and the standard deviations based on the triplicates were presented.

Both acetate and methanol contributed to the butyrate formation. Each of them likely contributed about half of the carbon in the butyrate formed. Assuming that the conversion of the yeast extract in MeAc and MeAc+E was similar to that in the blanks, there was about 10 $\mathrm{mMC}$ acetate and $3 \mathrm{mMC}$ butyrate produced from the converted yeast extract. Subtracting the fatty acid production in the blanks from MeAc and $\mathrm{MeAc}+\mathrm{E}$ excluded the contribution of the yeast extract to the organic acid formed. After this subtraction, there was actually more net acetate consumption ( $28 \mathrm{mMC}$ in $\mathrm{MeAc}$ and $32 \mathrm{mMC}$ in $\mathrm{MeAc}+\mathrm{E}$ ) than presented in Figure 3.2. This net acetate consumption with the subtraction of the blanks was comparable to the net methanol consumption, implying that acetate and methanol might have contributed equally to the butyrate formation. It is important to address the contribution of acetate to the 


\section{Chapter 3}

butyrate formation here, since acetate is the main component in the acidified organic waste, a cheap primary feedstock to chain elongation.

Consumption of both acetate and methanol as substrates for butyrate production indicated the feasibility of methanol chain elongation converting single (methanol) and double carbon (acetate) molecules to longer carbon molecules up to butyrate (four carbons); however, molecules with longer carbon chain like caproate (six carbons), i.e. MCFAs, were not observed in this $1^{\text {st }}$ batch experiment. Also, odd-chain fatty acids were not detected in any batches of the $1^{\text {st }}$ batch experiments. As an accumulation of ethanol was not detected in all the batches, it was unlikely that ethanol was produced and used for chain elongation in the $1^{\text {st }}$ batch experiment. It is possible that methanol chain elongation occurred using a 2-carbon unit molecule instead of a single carbon unit molecule. It is likely that two molecules of methanol were converted into one molecule of acetyl-CoA, which was then used for chain elongation. Such chain elongation mechanism was also proposed previously ${ }^{112}$. However, to authors' current knowledge, this chain elongation mechanism has not been proven yet.

\subsubsection{Mixed culture microbiome from a previous ethanol chain elongation was shaped to} perform methanol chain elongation

Effect of the pure culture E.limosum addition to the methanol chain elongation and its product formation were tested in the $1^{\text {st }}$ batch experiment. E.limosum was known to perform chain elongation of methanol, $\mathrm{CO}_{2}$ and/or acetate in a pure- and a co-culture environment, producing butyrate and caproate $47,55,110$. The addition of E.limosum was expected to stimulate the methanol chain elongation, leading to more methanol and acetate consumption and butyrate production than the batches without such addition. However, based on Figure 3.2 , the amounts of butyrate produced in the batches with and without E.limosum addition were in a similar range regardless of the various conditions tested. There are two possible explanations to this: One is that E.limosum existed in the mixed culture inoculum taken from 
the previous ethanol chain elongation experiment, and after the 21-day incubation it was enriched and able to carry out methanol chain elongation; the other possible explanation is that another unknown microorganism rather than E.limosum existed in the mixed culture inoculum, which was responsible for the methanol chain elongation observed. In either case, the enrichment of the mixed culture inoculum was proven to be able to perform the methanol chain elongation in this study. Methanol was neither supplied nor observed in the previous ethanol chain elongation experiment from where the mixed culture inoculum was taken ${ }^{36}$. It is possible that a microorganism in the mixed culture inoculum could survive in the ethanol chain elongation bioreactor without a methanol supplement and was able to gradually adapt its metabolism to use methanol when methanol became available in the environment. For example, it is known that E.limosum and many other methylotrophic acetogenic bacteria are able to grow autotrophically on $\mathrm{CO}_{2}$ and $\mathrm{H}_{2}{ }^{115}$. In the previous ethanol chain elongation bioreactor where the mixed culture inoculum was taken from, $\mathrm{CO}_{2}$ was continuously supplied and $\mathrm{H}_{2}$ was produced during the ethanol chain elongation based on the metabolism of C.kluyveri ${ }^{36}$. Grootscholten et al. (2013) observed that the $\mathrm{CO}_{2}$ supplied and $\mathrm{H}_{2}$ produced were mostly converted into methane through hydrogenotrophic methanogenesis ${ }^{36}$. However, one cannot exclude that a methylotrophic microorganism like E.limosum might present in the mixed culture microbiome in the ethanol chain elongation bioreactor. This methylotrophic microorganism could have grown on $\mathrm{CO}_{2}$ and $\mathrm{H}_{2}$ autotrophically and possibly produced acetate as the metabolites in the ethanol chain elongation bioreactor ${ }^{55}$. In the present study, this methylotrophic microorganism might have been enriched in the $1^{\text {st }}$ batch experiment and subsequently carried out the methanol chain elongation to produce butyrate. This implies that the mixed culture microbiome in the previous ethanol chain elongation reactor could be shaped into a mixed culture microbiome that is capable of performing methanol chain elongation. 


\section{Chapter 3}

\subsubsection{Caproate production was observed in the prolonged experiment $(2-M e A c+E)$}

The MeAc+E of the $1^{\text {st }}$ batch experiment was replicated in the $2^{\text {nd }}$ batch experiment (as 2$\mathrm{MeAc}+\mathrm{E})$ to enrich the biomass for later use, i.e. starting up the continuous methanol chain elongation bioreactor. In this replication, methanol was once (on day 7) elevated to $200 \mathrm{mMC}$ to prevent the methanol depletion. After the methanol elevation, caproate production (3 $\mathrm{mMC}$ ) was observed along with the increasing butyrate concentration up to $167 \mathrm{mMC}$ on day 13 as shown in Figure 3.3. In the meantime methanol and acetate were the main substrates consumed. The final butyrate production $(191 \mathrm{mMC})$ was far higher than the carbon that could be provided by the complete conversion of the added yeast extract ( $34 \mathrm{mMC})$. This result again confirmed the feasibility of methanol chain elongation as discussed in section 3.3.1.1; moreover, product formation (butyrate) as well as substrate consumption (acetate and methanol) was at least 2 times higher than those in MeAc and MeAc+E in the $1^{\text {st }}$ batch experiment (Figure 3.2). Interestingly caproate production was observed. The caproate formed could be produced from either methanol and acetate or methanol and butyrate through methanol chain elongation (Figure 3.3). Previously, caproate production from methanol and acetate/butyrate was reported in the pure culture E.limosum growing on methanol and acetate/butyrate as aforementioned in the introduction of the present study ${ }^{47,55,110}$. However, all these mentioned studies employed a pure culture E.limosum. This study, on the other hand, demonstrated the caproate production $(3 \mathrm{mMC})$ from methanol and acetate using a mixed culture microbiome. To authors' current knowledge, this result has not been reported in the previous studies. 


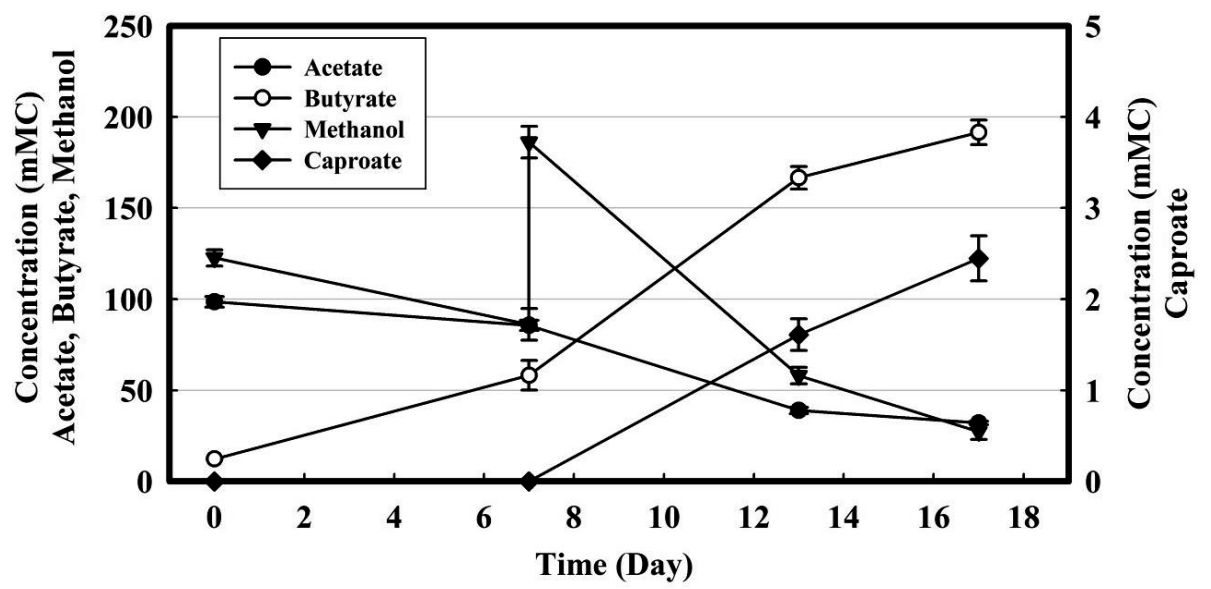

Figure 3.3. The concentration profile of the $2^{\text {nd }}$ batch experiment $(2-\mathrm{MeAc}+\mathrm{E})$. The $2-$ MeAc+E was incubated for 17 days, and then used as the inoculum for the continuous methanol chain elongation experiment. Methanol was added again on day 7 to prevent the methanol depletion. Caproate production was observed since day 13; it increased with the increasing butyrate production.

Many previous studies reported the caproate formation in a mixed culture fermentation when ethanol and/or hydrogen were available in the environment, i.e. ethanol chain elongation. However, the caproate production in the present study was unlikely produced through an ethanol chain elongation process. An accumulation of either ethanol or hydrogen was not detected though both ethanol and hydrogen were constantly monitored during the entire incubation period. Methanol chain elongation was another possible process responsible for the caproate production observed. The inoculum used for 2-MeAc+E may contain E.limosum. E.limosum is by far the only known microorganism capable of synthesising caproate from methanol, $\mathrm{CO}_{2}$ and SCFAs like acetate and butyrate ${ }^{47,55}$. It was logical to hypothesise that E.limosum or an unknown microorganism with a similar metabolism was present in 2-MeAC and responsible for the caproate production. Based on the aforementioned 


\section{Chapter 3}

pure culture studies on E.limosum, it seems that a certain threshold butyrate concentration, i.e. $400 \mathrm{mMC}$, was required to stimulate the caproate formation. In the present study, the butyrate concentration was only $160 \mathrm{mMC}$. However, in the previous study the methanol concentration (100 mMC) was lower compared with the concentration used in 2-MeAc (200 mMC) when caproate was observed. It is not yet known whether the elevation of methanol concentration could trigger or stimulate the caproate formation with a lower butyrate concentration $(<400 \mathrm{mMC})$. It cannot be excluded that another microorganism rather than E.limosum produced the caproate from methanol and SCFAs (either acetate or butyrate) even with lower SCFA concentrations, although such microorganism has not been reported yet. Yeast extract might be another possible source for the caproate production; however, yeast extract is less likely the source of caproate production as such possibility was not observed in the blanks in the $1^{\text {st }}$ batch over the entire 21 -day incubation.

From these two batch experiments, three conclusions could be drawn: First, chain elongation of acetate and methanol with a mixed culture microbiome, i.e. methanol chain elongation, was proven to be feasible. The main product from methanol chain elongation in the present study was butyrate with a concentration up to $191 \mathrm{mMC}$. Second, an enrichment of the mixed culture inoculum from previous ethanol chain elongation bioreactor was able to perform methanol chain elongation producing butyrate from acetate and methanol. Addition of pure culture E.limosum did not further enhance methanol chain elongation. Third, caproate production was observed and probably produced from methanol and acetate/butyrate. E.limosum is speculated to be responsible for the caproate formation observed; however, further research is needed to reveal the microorganism responsible for the caproate production in the methanol chain elongation and subsequently to enhance its production. 


\subsubsection{Methanol chain elongation in the continuous operation}

3.3.2.1 Continuous butyrate production from methanol chain elongation on methanol and acetate

Continuous butyrate production through methanol chain elongation on acetate and methanol was shown to be feasible in the UAB at an HRT of around 36 hours as shown in Phase II and Phase III (see Figure 3.4). In this first demonstration of continuous methanol chain elongation, a butyrate production rate up to $68 \mathrm{mMC} /$ day $(1.5 \mathrm{~g} / \mathrm{L} / \mathrm{day})$ was reached. The butyrate concentration (103 mMC, see Figure S3.5) in the continuous operation was not as high as such in the 2-MeAc (191 mMC), and the caproate production was not observed. In both Phase II and Phase III acetate and methanol were the major consumed substrates. Butyrate was the main product; $\mathrm{CH}_{4}$ and $\mathrm{CO}_{2}$ were the by-products. Figure 3.5 presents the average production and consumption rates of all the substrates and products in Phase II and Phase III, respectively. In Phase II, the butyrate production rate was similar to the sum of the acetate and methanol consumption rates, implying that butyrate was mainly produced from methanol and acetate. Trace amount of $\mathrm{CO}_{2}$ was produced, which was identical with the observation in the methanol chain elongation batch experiments. Based on the result of the $1^{\text {st }}$ batch experiment, the conversion of yeast extract may contribute maximal $3 \mathrm{mMC}$ to the butyrate formation. This did not change the fact that acetate and methanol both contributed to the butyrate formation in the continuous methanol chain elongation. In Phase III the same conclusion could be drawn except that the butyrate production in Phase III was slightly higher than that in Phase II especially from day 35 to day 45. The difference between Phase II and Phase III was elaborated in the section 3.3.2.2 in the present study. 


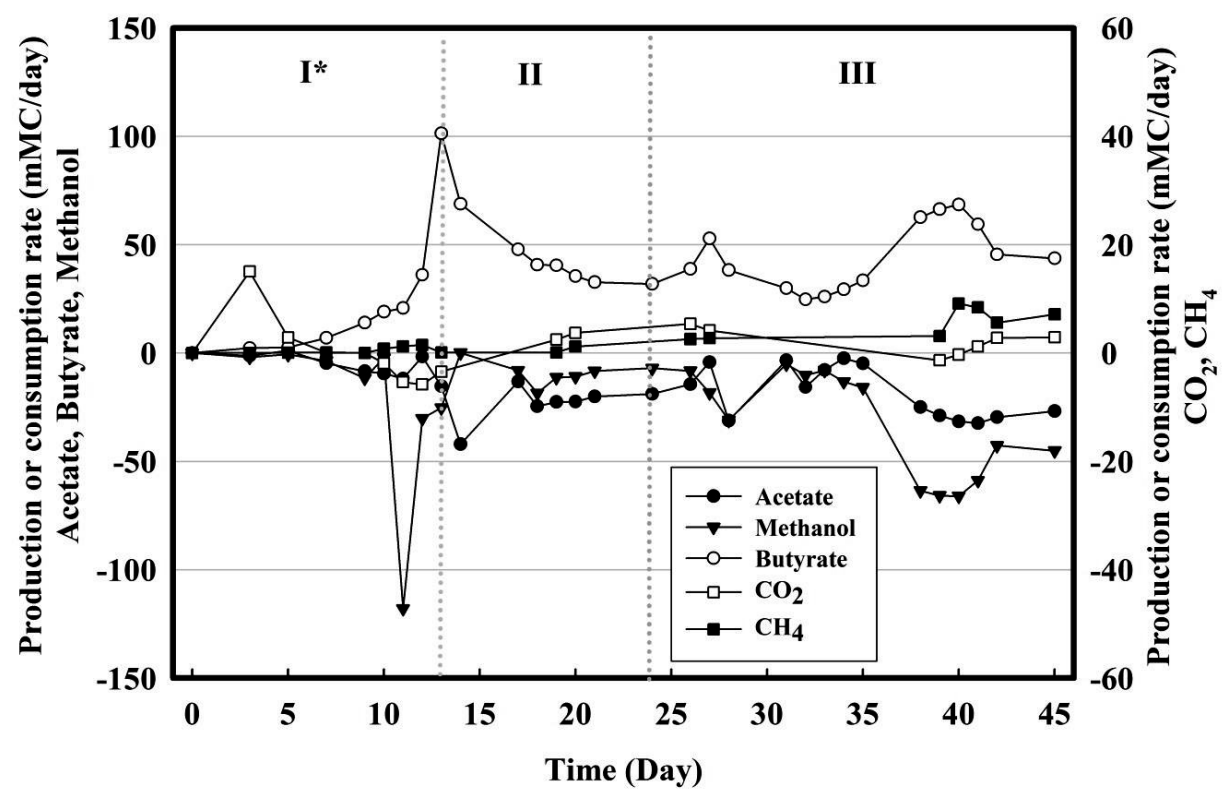

Figure 3.4. The profile of the main production and consumption over the whole continuous methanol chain elongation experiment for 45 days. The continuous butyrate production from methanol and acetate was observed in all phases. *As Phase $\mathrm{I}$ is in batch mode, the production and consumption rates in Phase I were calculated differently compared with the other phases (See SI3). Moreover, caproate was detected on day 12 and 13 but not included in this figure. More information about the caproate production can be found in Figure S3.5.

It was noticed that $\mathrm{CO}_{2}$ was consumed in Phase I (batch mode for starting up) of the UAB operation (see Figure 3.4 and 3.5). In the $1^{\text {st }}$ batch experiment $\mathrm{CO}_{2}$ was not used but produced. In Phase II and Phase III of the UAB operation (continuous mode) $\mathrm{CO}_{2}$ was also mostly produced (Figure 3.4). The $\mathrm{CO}_{2}$ consumption observed in Phase I could relate to the $\mathrm{CO}_{2}$ partial pressure in the headspace. In the $1^{\text {st }}$ batch experiment the $\mathrm{CO}_{2}$ partial pressures in all batch bottles were around 22-24 kPa (see Figure S3.3). In Phase I of the UAB operation the $\mathrm{CO}_{2}$ partial pressure in the headspace was always higher than $30 \mathrm{kPa}$ (Figure S3.4). Higher $\mathrm{CO}_{2}$ partial pressure in the headspace resulted in higher $\mathrm{HCO}_{3}{ }^{-}$concentration in the 
fermentation broth, which may stimulate microorganisms to use $\mathrm{HCO}_{3}{ }^{-}$as a substrate. The higher $\mathrm{CO}_{2}$ partial pressure could be explained by the way of $\mathrm{CO}_{2}$ supplementation: In the $1^{\text {st }}$ batch experiment $\mathrm{CO}_{2}$ was injected into the headspace at the beginning of the experiment, while in the $\mathrm{UAB} \mathrm{CO}_{2}$ was bubbled through the reactor continuously. $\mathrm{CO}_{2}$ was believed to be an essential substrate for ethanol chain elongation ${ }^{41}$. Most previous ethanol chain elongation studies supplied $\mathrm{CO}_{2}$ to sustain the microbial growth. Therefore, $\mathrm{CO}_{2}$ was also supplied in the present study. However, in the present study $\mathrm{CO}_{2}$ was always produced instead of consumed except in Phase I of the UAB operation. In the future it can be further tested whether $\mathrm{CO}_{2}$ supplement is needed in methanol chain elongation.

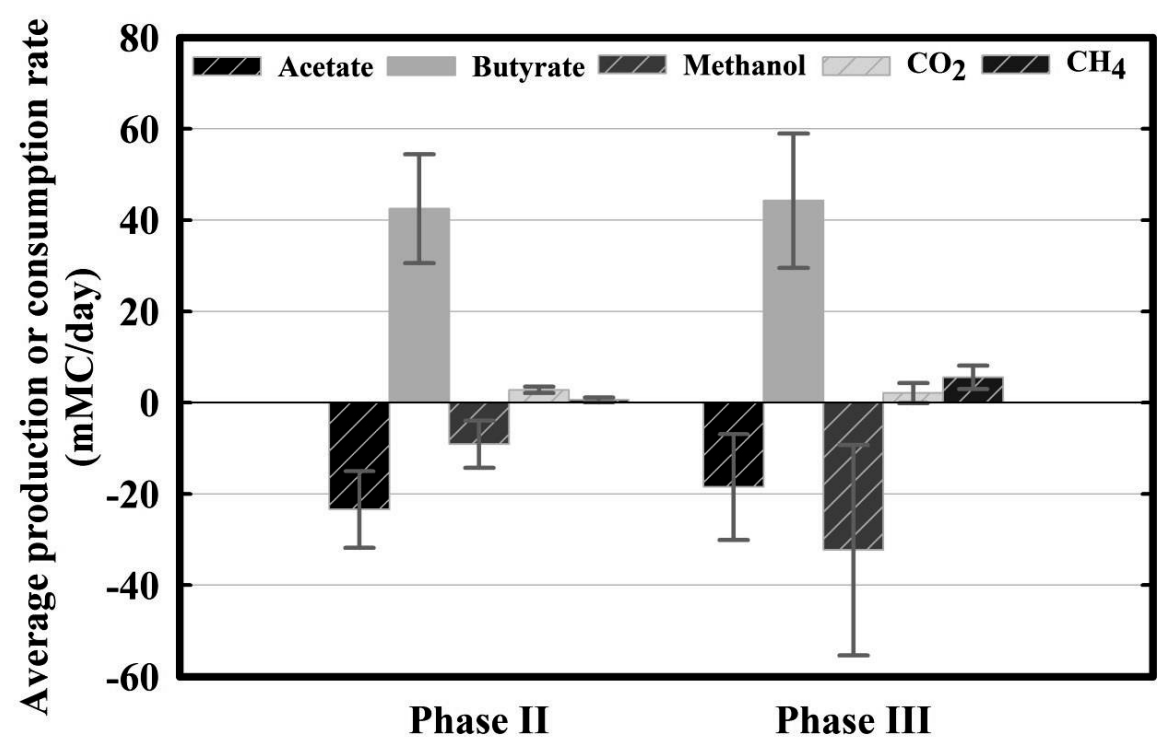

Figure 3.5 The average production and consumption rates of the main substrates and products involved in the continuous methanol chain elongation operation in Phase II and Phase III. Caproate was not detected during the entire Phase II and III. Therefore caproate was not shown in this figure. 
3.3.2.2 Increase of $p H$ increased the butyrate production, but changed the substrate consumption pattern

In Phase III, the increase of $\mathrm{pH}$ slightly increased the butyrate and $\mathrm{CH}_{4}$ production. The pattern of the substrate utilisation for the butyrate formation was also changed along with the pH change from Phase II to Phase III. In Phase II acetate was the main carbon source for the butyrate formation, while in Phase III methanol contributed more carbon than acetate to the formation of butyrate. Meanwhile, the $\mathrm{CH}_{4}$ production gradually increased since day 39 (Phase III; see Figure 3.4 and Figure S3.4). The $\mathrm{CO}_{2}$ partial pressure as well as the $\mathrm{CO}_{2}$ production decreased (Figure 3.4 and Figure S3.4), which may be attributed to the increase of methane production. The maximal carbon consumed for the methane production was about 4.2 to $5.3 \%$ of the total carbon input and lasted from day 40 to 45 (Figure 3.5).

The increase of $\mathrm{CH}_{4}$ production observed in Phase III could be attributed to the increase of $\mathrm{pH}$. In the period when the $\mathrm{CH}_{4}$ production increased (day 40 to 45 ), the acetate consumption gradually increased. In the meantime the methanol consumption was also increased and exceeded the amount of acetate consumption, resulting in an overall decrease in the carbon balance (Figure S3.2). A similar phenomenon was reported in the previous studies regarding the metabolism of E.limosum. A pure culture E.limosum growing on methanol, $\mathrm{CO}_{2}$ and acetate shifted its metabolism to consume acetate instead of producing acetate when $\mathrm{CO}_{2}$ fixation was rate-limiting and the acetate concentration was sufficient $(>190 \mathrm{mMC})^{116,117}$. An increase in the methanol consumption occurred along with this shift in the metabolism of the E.limosum to an acetate-consuming homobutyric fermentation. Pacaud et al. (1986) suggested that the extra methanol consumed was dissimilated into $\mathrm{CO}_{2}$ for maintaining a constant $\mathrm{NAD}(\mathrm{P}) \mathrm{H}_{2} / \mathrm{NAD}(\mathrm{P})$ metabolites pool, and part of the extra carbon loss might end up in the biomass formation ${ }^{117}$. In Phase III of the present study, the continuous supply of 200 $\mathrm{mMC}$ acetate together with the lower $\mathrm{CO}_{2}$ partial pressure in the headspace (possibly due to the increased hydrogenotrophic methanogenic activities) might trigger a similar shift in the 
metabolism of a part of the microorganism responsible for the butyrate production in the continuous methanol chain elongation. Based on the Phase III of this continuous methanol chain elongation, increasing the $\mathrm{pH}$ of the fermentation might be effective in stimulating the butyrate formation in methanol chain elongation; however, it also increased the consumption of methanol and induced an extra carbon loss in terms of the increased methane production and the potentially increased biomass formation. The effect of changing $\mathrm{pH}$ on the overall carbon efficiency should be further tested and evaluated.

Overall, the present study demonstrated the feasibility of methanol chain elongation, both in a batch and a continuous operation. Optimisation strategies to elevate the butyrate production rate and to stimulate the caproate production in methanol chain elongation are needed. The optimisation strategies that were previously applied to ethanol chain elongation could be tested in the future methanol chain elongation studies. For example, Grootscholten et al. (2013) reduced the HRT of an ethanol chain elongation to increase the MCFA production rate ${ }^{35}$. Agler et al. (2011) decreased the $\mathrm{pH}$ of ethanol chain elongation to reduce the methane production and employed an in-line liquid-liquid extraction to continuously remove the MCFAs produced for avoiding the potential product inhibition ${ }^{37}$. These optimisation strategies, especially reducing the HRT and continuously removing the product, may be effective in elevating the butyrate production rate and concentration in methanol chain elongation. The elevation of the butyrate concentration in the continuous methanol chain elongation may stimulate the caproate formation ${ }^{47}$. A higher butyrate production rate may also make methanol chain elongation an attractive technology to be combined with the ethanol chain elongation to reduce the ethanol dose required for synthesising MCFAs. Moreover, the microorganisms responsible for the butyrate and caproate formation in this study should be revealed in the future studies. In this study many discussions on the results were based on the assumption that the microorganism responsible for the methanol chain 
Chapter 3

elongation observed was physiologically identical or similar to E.limosum. However, this might not be the case. Further study should try to identify the microorganism responsible for methanol chain elongation and to understand its metabolism among the mixed culture microbiome in order to steer the mixed culture microbiome towards the desired production behaviour. 


\section{Support Information for Chapter 3 (SI3)}

\section{Batch preparation procedure}

Serum bottles $(250 \mathrm{ml})$ were filled with $100 \mathrm{ml}$ growth medium described in section 3.2.1 to perform the batch test. No buffer was added to the batches as preliminary tests showed that $\mathrm{pH}$ can be maintained at 5.9- 6.1. These batch bottles were sealed with rubber stopper and capped with aluminium caps. The headspaces were then flushed with $80 \% \mathrm{~N}_{2}$ and $20 \% \mathrm{CO}_{2}$ for 5 times to the defined composition (Table 3.1) with a final pressure of $150 \mathrm{kPa}$. Each bottle was inoculated with either a $5 \mathrm{ml}$ mixed culture inoculum from previous Ethanol chain elongation bioreactor $(\mathrm{B}, \mathrm{Me}$ and $\mathrm{MeAc})$ or with a $2.5 \mathrm{ml}$ mixed culture inoculum plus a 2.5 $\mathrm{ml}$ pure culture E.limosum. The inoculated batch bottles are subsequently incubated at $35^{\circ} \mathrm{C}$ in a rotating shaker (120 rpm) for 21 days.

The pure culture E.limosum (ATCC 8486) was purchased from DSMZ, Germany. The purchased strain was first incubated in a pre-sterilised serum bottle $(250 \mathrm{ml})$ at $35^{\circ} \mathrm{C}$. The preparation procedure was identical to the procedure used for preparing the $1^{\text {st }}$ and $2^{\text {nd }}$ batch. The serum bottle was filled with the growth medium $(150 \mathrm{ml})$ containing the aforementioned composition as well as acetate and methanol. The growth medium, except the vitamin B solution, the trace element solution and the yeast extract, was sterilised using an autoclave $\left(120^{\circ} \mathrm{C}\right.$ and $140 \mathrm{kPa}$ for 20 minutes) prior to the incubation. The vitamin B solution, the trace element solution and the yeast extract was sterilised by the filtration using a 20 um cellulose paper filter. The initial headspace composition consisted of $80 \% \mathrm{~N}_{2}$ and $20 \% \mathrm{CO}_{2}$. In this pure culture incubation samples were taken from the broth to examine the metabolites, and butyrate was the main metabolites observed (data not shown). The sampling procedure was carried out in a sterilised fume hood to avoid the possible contamination. The fermentation broth taken from this pure culture incubation was first centrifuged. The supernatant was removed, and the pellet was washed with the sterilised growth medium. The centrifuging and 


\section{Chapter 3}

washing were repeated for three times to lower the organic acid and methanol concentration in the inoculum.

\section{Calculation of $\mathrm{CO}_{2}$ input and outputs in the $\mathrm{UAB}$}

Calculation of $\mathrm{CO}_{2}$ outputs in different forms in the UAB is described here. Gaseous $\mathrm{CO}_{2}$ ( $240 \mathrm{ml} /$ day) is the only form of $\mathrm{CO}_{2}$ input used in the $\mathrm{UAB}$. Gaseous $\mathrm{CO}_{2}$, dissolved $\mathrm{CO}_{2}$ $\left(\mathrm{dCO}_{2}\right), \mathrm{H}_{2} \mathrm{CO}_{3}$ and $\mathrm{HCO}_{3}{ }^{-}$are four possible outputs considered in the calculation. In all phases in continuous operation of methanol chain elongation, gaseous $\mathrm{CO}_{2}$ output is calculated based on the gas outflow data monitored by the gas counter (Ritter MGC-1 PMMA, Germany) and the gas composition identified with a gas chromatograph (Shimadzu GC-2010, Japan). In continuous operation, there was $\mathrm{CO}_{2}$ output in the form of $\mathrm{dCO}_{2}, \mathrm{H}_{2} \mathrm{CO}_{3}$ and $\mathrm{HCO}_{3}{ }^{-}$in the effluent of $\mathrm{UAB}$, i.e. these three forms of $\mathrm{CO}_{2}$ left the $\mathrm{UAB}$ together with the effluent. The amount of each form of $\mathrm{CO}_{2}$ in the effluent can be estimated by applying the calculation method used in previous study on E.limosum ${ }^{112}$, and everyday there was approximately 0.66 litre of effluent discharged from the UAB. The net $\mathrm{CO}_{2}$ change, i.e. $\mathrm{CO}_{2}$ consumed/produced, due to microbial activities in the UAB can therefore be calculated as shown in Figure 3.4.

\section{Calculation of production/consumption rates in continuous methanol chain elongation}

In Figure 3.5, the production/consumption rate in Phase I is calculated differently from the other phases as it was in batch operation while the others were in continuous operation. In batch operation, the production/consumption rate of a substance is calculated by using the formula below.

\section{Production/consumption rate of substance $x=(C x, t-C x, t-1) / H R T^{*} 24(m M C / d a y)$}

Where $\quad C x, t=$ the concentration of substance $x$ at day $t$ in $m M C$.

$C x, t-1=$ the concentration of substance $x$ at day (t-1) in $m M C$. $t=$ day of the sample taken (day) 


$$
\text { HRT }=\text { Hydraulic Retention Time, in this case } 36 \text { hours }
$$

In this case, the production/consumption rate is calculated by comparing the concentration of a substance on a sample point with such on the previous sample point, which is normally a day before. On the other hand, in Phase II and Phase III with the continuous operation, the production/consumption rate is calculated by using the formula below:

Production/consumption rate of substance $x=(C x, t-C i x, t) / H R T * 24$

Where $\quad C x, t=$ the concentration of substance $x$ at day $t$ in $m M C$.

Cix, $t=$ the concentration of substance $x$ in the medium fed at day $t$ in $m M C$.

In this case, the production/consumption rate is calculated by comparing the concentration of a substance in the broth on a sample point with the concentration of such substance in the medium fed into the reactor on the same sample point. 


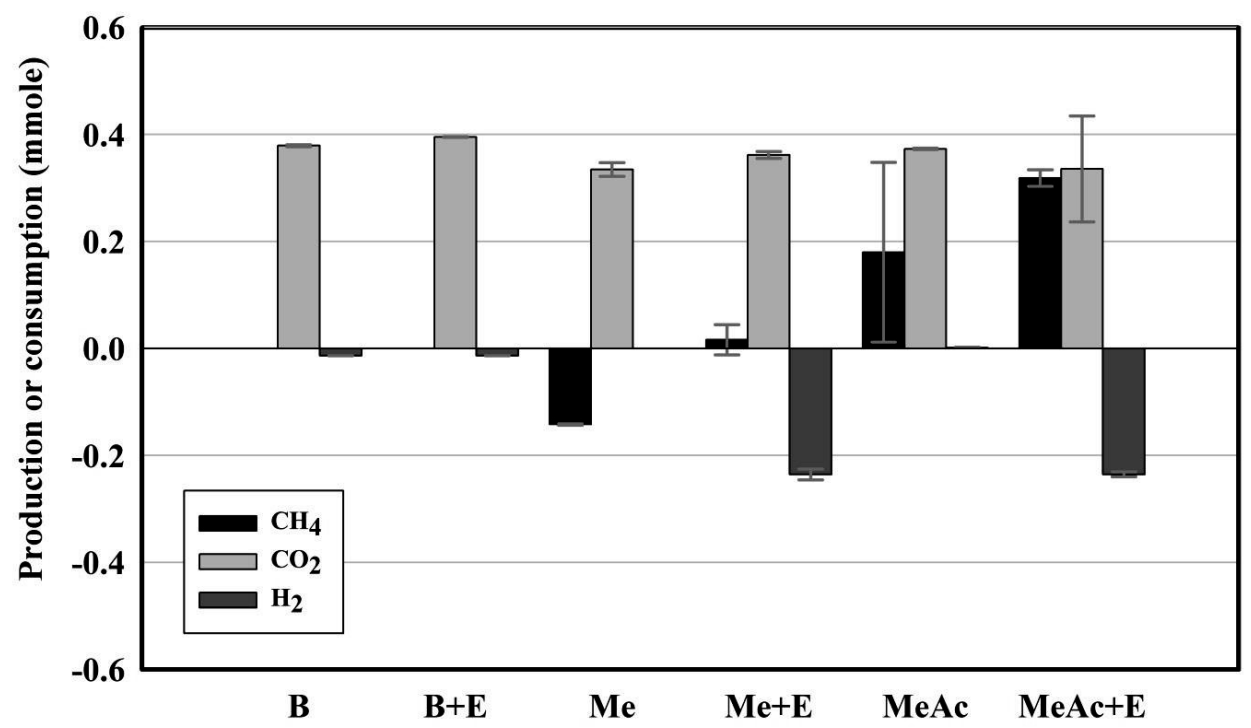

Figure S3.1. The production and consumption of the main gas component in the head space of each batch. All results presented were the mean value of the triplicate with the standard deviations. Net $\mathrm{CO}_{2}$ production was observed in all the batches. Consumptions of hydrogen and methane were observed in some batches; this might be caused by the impurities introduced during the gas flushing process prior to inoculation (See section 3.2). 
(A)

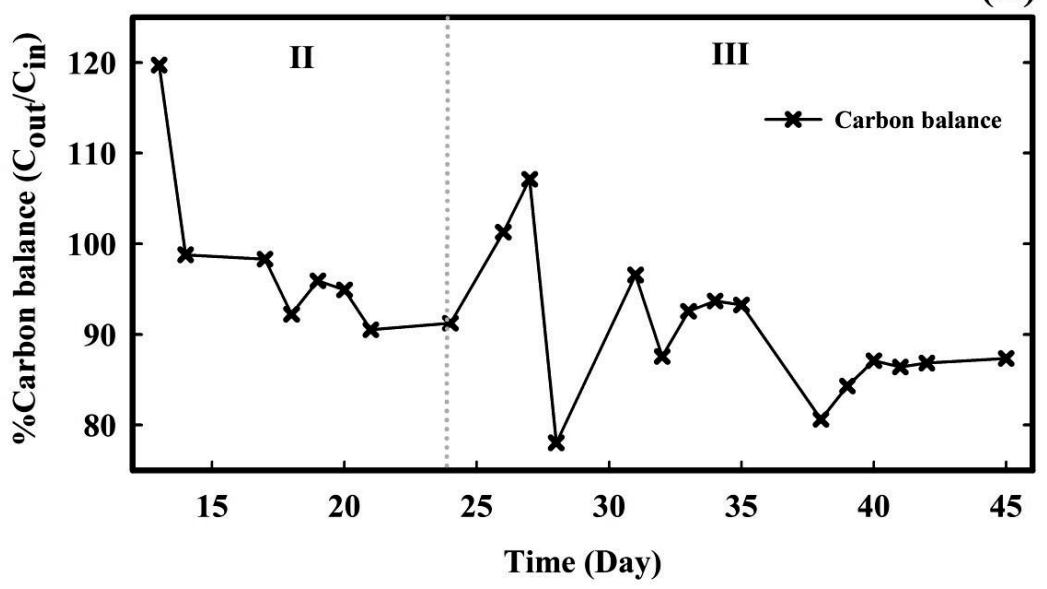

(B)

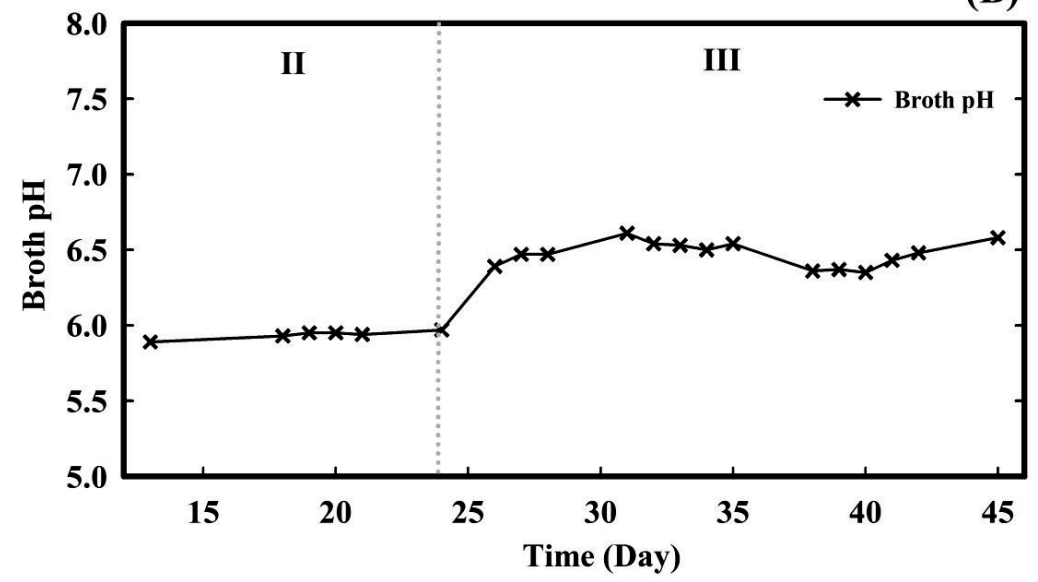

Figure S3.2. (A) The carbon balance and (B) the pH in Phase II and III during the continuous methanol chain elongation. In Phase III pH was controlled at $6.5 \pm 0.1$. $\mathrm{C}=$ Carbon. 


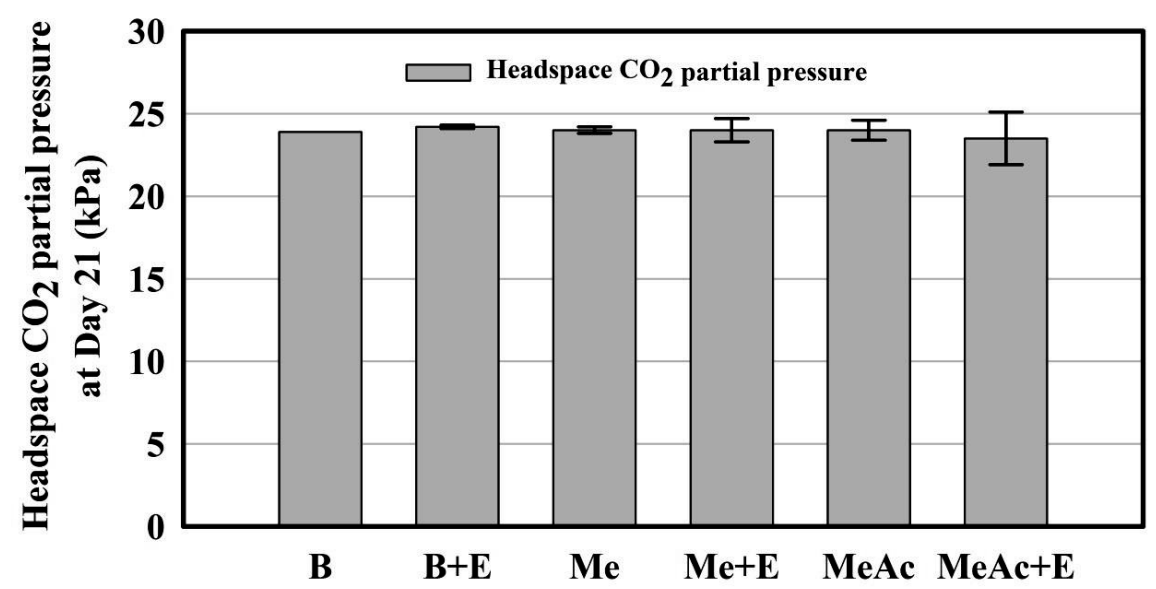

Figure S3.3. The $\mathrm{CO}_{2}$ partial pressure in the headspace of the $1^{\text {st }}$ batch experiment at day 21, the end of the experiment. For all batches $\mathrm{CO}_{2}$ partial pressure in the headspace reached the highest on day 21 . 


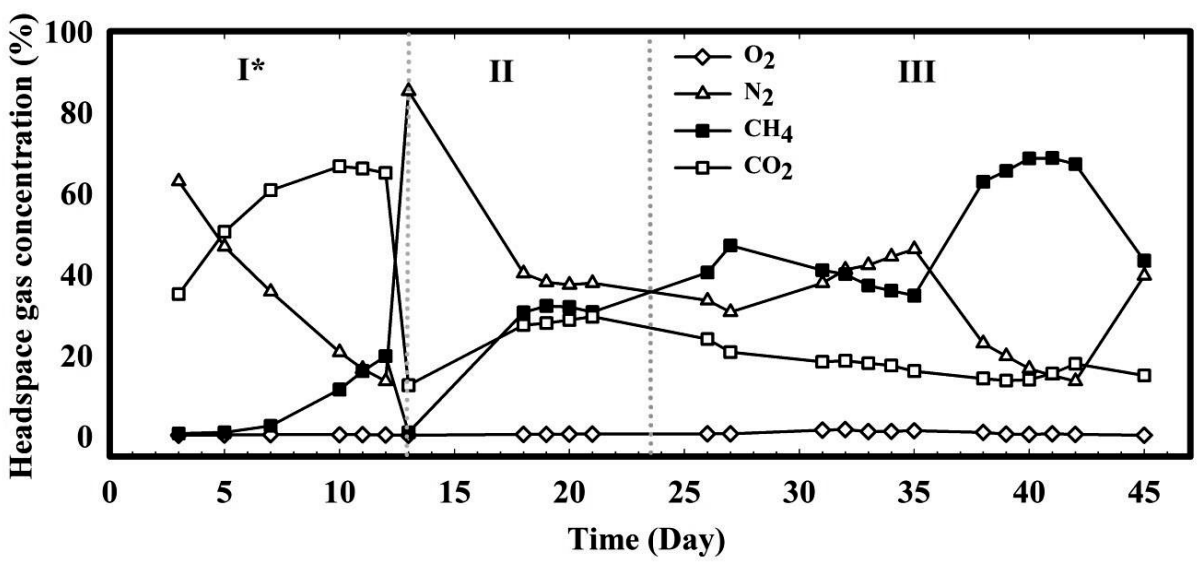

Figure S3.4. The headspace gas composition of the UAB during the 45-day continuous operation of methanol chain elongation. In the Phase III the methane production increased especially after day 35 . The headspace pressure was kept at the atmosphere pressure. On day 13 the headspace was flushed with the nitrogen gas due to the start of continuous operation. *Phase I was in a batch operation for starting-up methanol chain elongation. 


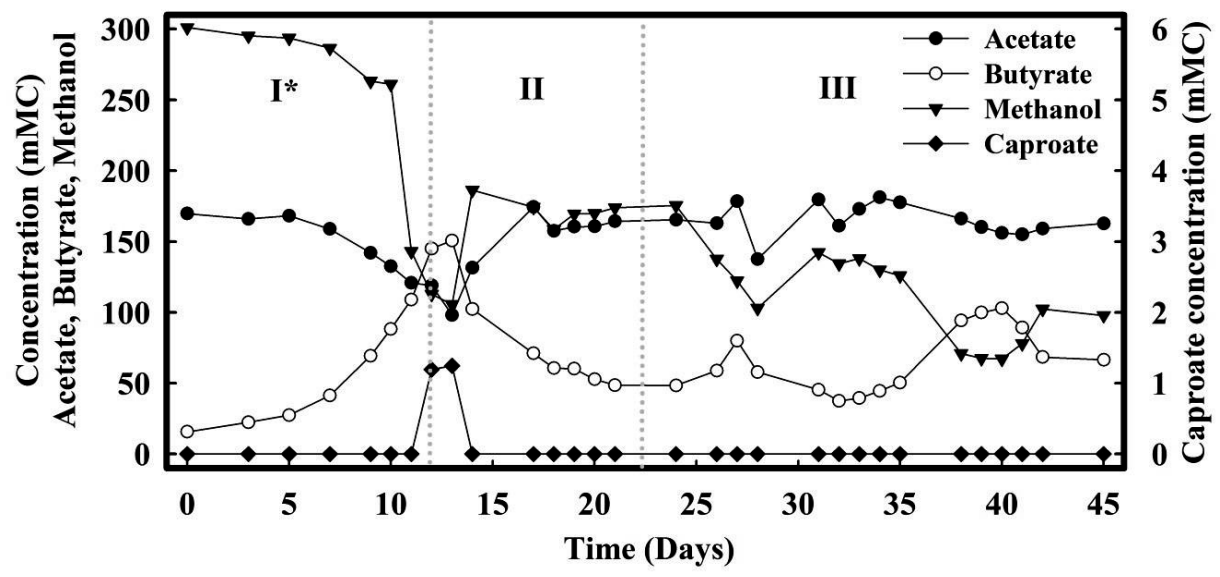

Figure S3.5. The concentration profile of the main substrates and products in the liquid phase (Fermentation broth) of the continuous methanol chain elongation for the whole 45day period. The caproate concentration was also included, but it was only observed in the batch operation phase (Phase I); the caproate formation observed on day 13 was the residual caproate from the batch operation of methanol chain elongation (Phase I). The HRT was longer than 24 hours, so the caproate produced on day 12 was not completely washed out or diluted on day 13. *Phase I was in a batch operation for starting-up methanol chain elongation. 
Methanol chain elongation: Proof-of-principle 


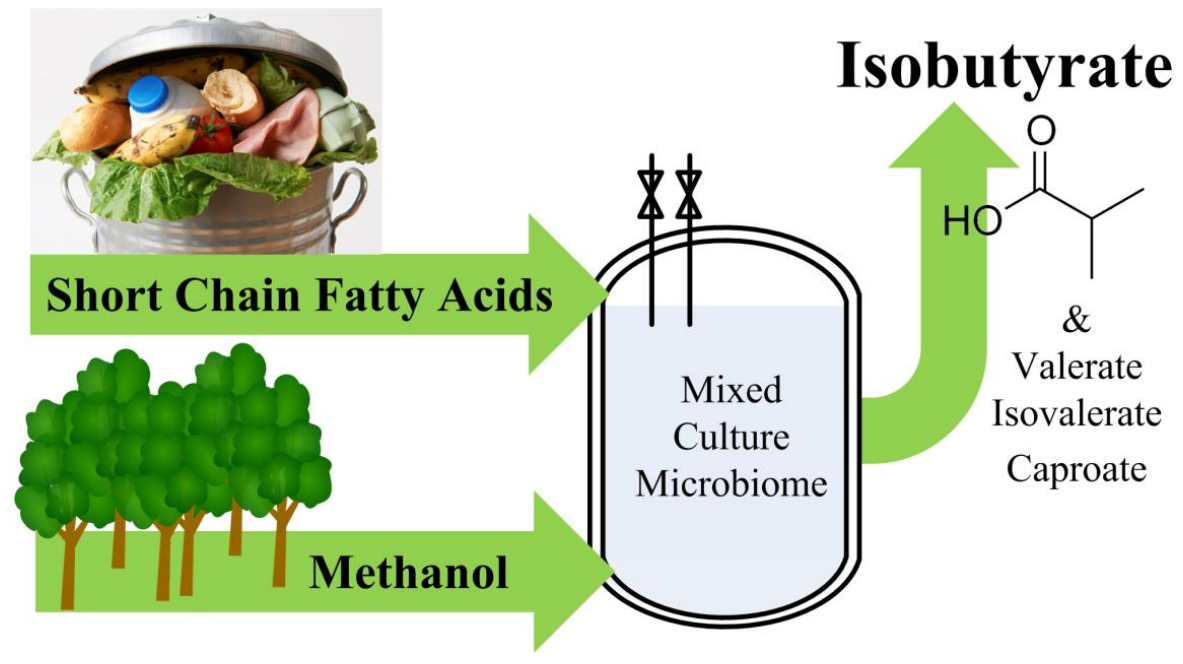




\title{
Chapter 4. Isobutyrate biosynthesis via methanol chain elongation
}

\begin{abstract}
Isobutyrate is a platform chemical that is currently produced from a non-renewable fossilbased feedstock. This study aimed at developing a renewable isobutyrate production process by using methanol chain elongation, a novel bioprocess that uses organic waste as primary feedstocks and an undefined reactor microbiome as the catalyst. A continuous anaerobic bioreactor experiment was first performed using a synthetic medium containing acetate, butyrate and methanol, all of which are common derivatives from organic residues. Continuous isobutyrate (2.0 g/L.day) and caproate formation ( 0.2 g/L.day) from methanol, acetate and butyrate were demonstrated. A batch test to synthesis isobutyrate from a real organic waste i.e. acidified supermarket waste (ASW) was performed. Up to $6.2 \mathrm{~g} / \mathrm{L}$ isobutyrate was produced which accounted for $63 \%$ of all identified products. As such, a proof-of-principle for isobutyrate production from organic waste via methanol chain elongation was demonstrated. The continuous accumulation or supply of butyrate, the suppression of methanogenic activity and methanol addition were shown to be of use to provide conditions for isobutyrate formation.
\end{abstract}

A modified version of this chapter was published as: Chen, W.S.; Huang, S.; Strik, D. P. B. T. B.; Buisman, C. J. N. Isobutyrate biosynthesis via methanol chain elongation: Converting organic wastes to platform chemicals. Journal of Chemical Technology \& Biotechnology 2016. 


\subsection{Introduction}

The current fuel and chemical production heavily relies on non-renewable fossil resources, which has led to environmental concerns such as global warming and air pollution. Bio-based production systems using biomass as a renewable feedstock, e.g. bioethanol from sugarcontaining crops and biodiesel from oil crops, have been implemented as alternatives to the fossil-based production system. These crop-based systems expand rapidly and are estimated to occupy 385 million hectares of plantation globally by 2050 in the most intensive scenario $^{118}$. This enormous demand for land resources raises the concern towards the competition with food production ${ }^{13}$ and the deterioration of the natural systems due to the emissions from land-use change ${ }^{9}$. Organic waste is an emerging bio-based feedstock for fuel and chemical production that has a lower land requirement. Using organic waste as feedstock can avoid the fuel versus food competition and concurrently fulfil the waste treatment for these otherwise wasted organic materials. Moreover, using organic waste can also reduce the feedstock cost due to its negative economic value. Recovering fuel and chemical precursors from organic residues also yields higher economic values than recovering energy from

organic residue which is currently implemented ${ }^{62,63}$. Research effort on the valorisation of organic residues into chemical and fuel precursors has therefore gradually increased over time $^{119}$.

Chain elongation is a recently established bioprocess that can employ a mixed culture to recover value-added chemicals and fuel precursors from organic waste ${ }^{37-40,}$.77. Chain elongation has been applied to, for example, solid organic waste such as organic fraction of municipal solid waste (OFMSW). In such case, a biochemical pre-treatment, i.e. hydrolysis and acidification, is first used to degrade and homogenise complex organic matters into basic building blocks including Short Chain Fatty Acids (SCFAs; saturated carboxylic acid with 2 to 5 carbons), $\mathrm{H}_{2}$ and $\mathrm{CO}_{2}{ }^{30}$. These building blocks then enter the chain elongation process to 
be elongated with an externally supplied electron donor such as ethanol ${ }^{32,}{ }^{37}, \mathrm{H}_{2}{ }^{48}$ or methanol $^{90}$ to Medium Chain Fatty Acids (MCFAs; saturated carboxylic acid with six to twelve carbons). Alternatively, chain elongation can also be directly applied to liquid waste streams such as a yeast-fermentation beer which already contains ethanol and other essential substrates for chain elongation ${ }^{37,66}$. In either case, caproate with a six-carbon chain is the main end-product. Caproate is an emerging platform chemical with diverse applications such as feed additives and precursors to fragrances, pharmaceuticals, antimicrobial, etc ${ }^{66}$. Caproate is also by far the most widely-studied product of chain elongation due to its high economic value, its high production specificity and the easier separation of it from the fermentation broth. A pilot plant (ChainCraft B.V.) has been implemented to convert supermarket waste into commercially viable caproate ${ }^{33}$. As the caproate production through chain elongation is approaching the commercialisation, the future research on chain elongation may gradually shift from producing caproate, which has a relatively small market, to other platform chemicals that have larger markets ${ }^{33}$.

The present study aims at exploring potential novel products of chain elongation besides caproate. We have identified isobutyrate as one of the interesting chemicals that are to be produced through chain elongation. Isobutyrate, though not a MCFA, is a useful platform chemical that can be used as a precursor for e.g. (1) Methyl Methacrylic Acid (MMA) that is used for production of transparent thermoplastics with an annual market size of up to 2.2 million tonnes ${ }^{57,120,121},(2)$ sucrose acetate isobutyrate that is used as an emulsifier in many commodities with an annual market size of up to 100,000 tonnes ${ }^{57,122}$ or (3) 3hydroxyisobutyric acid that can be used for synthesis of vitamins, antibiotics and many other bioactive compounds ${ }^{123}$. A recent study also showed that isobutyrate could be biologically reduced in a syngas fermentation process to yield isobutanol ${ }^{124}$, a commercially applied fuel substitute that has a superior performance over ethanol ${ }^{125-127}$. All these applications make 


\section{Chapter 4}

isobutyrate an interesting platform chemical. Currently, isobutyrate is manufactured via a chemical route that requires a fossil-based feedstock, an energy-intensive process and a strongly acidic catalyst that is hazardous to the environment ${ }^{57,122}$. Biosynthesis of isobutyrate has been reported but only in two previous studies that used glucose as the main substrate $e^{57}$ ${ }^{122}$, which may still raise the concerns towards the fuel versus food competition. Furthermore, these two studies both employed metabolically engineered microorganisms as the biocatalyst, which made them difficult to be applied on organic waste ${ }^{33}$.

Although isobutyrate formation was previously observed during the anaerobic degradation of organic matters in sediments ${ }^{128}$, in organic waste ${ }^{129-131}$ and in wastewater ${ }^{132}$ under nonsterile conditions, isobutyrate production from organic waste has never been demonstrated. This is due to that isobutyrate formation in these previous studies is mostly temporal, i.e. cannot be sustained. The isobutyrate formation in these studies was from the biological isomerisation of butyrate (hereinafter referred to as bio-isomerisation) ${ }^{133}$ under the condition that methanogenic activity was suppressed and butyrate accumulated (or was supplied). When the methanogenic activity was restored, the formed isobutyrate was de-isomerised into butyrate which further degraded into acetate, $\mathrm{H}_{2}$ and $\mathrm{CO}_{2}$ and eventually methane ${ }^{128-135}$. The obstacle to achieving isobutyrate production from organic waste, therefore, lies in how to sustain or even further stimulate bio-isomerisation during the anaerobic degradation of organic waste. Chain elongation can be of use to overcome this obstacle. When methanol is used in chain elongation as the electron donor (hereinafter referred to as methanol chain elongation), butyrate is continuously formed via chain elongation of methanol and acetate ${ }^{90}$, which may be used to provide sufficient butyrate for bio-isomerisation. At the meantime, the methanogenic activity could be suppressed due to the presence of organic acids in high concentrations $^{136,137}$. A novel bioprocess for isobutyrate production from organic waste could, in theory, be developed by integrating the bio-isomerisation of butyrate and methanol 
chain elongation, in which isobutyrate formation is no longer temporal. Moreover, comparing with the current isobutyrate production process, isobutyrate production via methanol chain elongation has advantages including the use of a renewable feedstock i.e. organic waste, the use of a self-regenerating biocatalyst and the possibility to work under non-sterile conditions due to the usage of a mixed culture.

In the present study, we aimed at demonstrating the proof-of-principle for isobutyrate production from organic waste via methanol chain elongation. A continuous anaerobic bioreactor was first operated with a synthetic medium to investigate the effectiveness of strategies for achieving isobutyrate biosynthesis from methanol and SCFAs via methanol chain elongation. A batch test on acidified supermarket waste (ASW) was then carried out to examine the feasibility of using a real organic waste for isobutyrate production via methanol chain elongation. The isobutyrate production was evaluated on concentration, rate and product distribution. The possible mechanisms behind the isobutyrate formation were further discussed. A comparison with the previous isobutyrate biosynthesis through synthetic metabolic pathways was also discussed.

\subsection{Methodology}

\subsubsection{The continuous methanol chain elongation on a synthetic medium.}

An anaerobic bioreactor was used for performing the continuous methanol chain elongation experiment on a synthetic medium. The setup of the anaerobic bioreactor is identical to the one used in a previous methanol chain elongation study ${ }^{90}$ (Figure S4.1) except for the use of polyurethane cubes. In the present study polyurethane cubes (Recticel, Belgium) were added in the bioreactor to increase the biomass retention, which was also used in several previous ethanol chain elongation studies ${ }^{35,36}$. The entire continuous experimental period consisted of five phases (Table 4.1). In Phase I-III, methanol feeding rate was increased from 60 $\mathrm{mMC} /$ day (Phase I) to $120 \mathrm{mMC} /$ day (Phase II) and $180 \mathrm{mMC} /$ day (Phase III; mMC refers to 


\section{Chapter 4}

millimolar carbon) to enhance the butyrate production rate, which might subsequently initiate the isobutyrate formation. In Phase IV, methanol feeding rate was brought back to $120 \mathrm{mMC}$ to restore the microbial activity. In Phase V, $480 \mathrm{mMC}$ /day butyrate was directly supplied to stimulate the isobutyrate formation. Vitamin B12 (i.e. cyanocobalamin) was increased from $13 \mu \mathrm{g} / \mathrm{L}$ to $400 \mu \mathrm{g} / \mathrm{L}$ to ensure the sufficient availability of the essential coenzyme required for catalysing both the methanol chain elongation ${ }^{138}$ and the isomerisation of butyrate ${ }^{134}$. The hydraulic retention time (HRT) was 40 hours in all phases. $\mathrm{pH}$ was not controlled during the entire 60-day operation. Internal recirculation $(150 \mathrm{ml} /$ minute $)$ was carried out with a pump (Watson-Marlow ${ }^{\circledR} 323 \mathrm{Dz}, \mathrm{UK}$ ) for continuously mixing the fermentation broth. The temperature of the bioreactor was controlled at $35{ }^{\circ} \mathrm{C}$ using a water bath (Fisher Scientific Polystat 37, USA). Gaseous $\mathrm{CO}_{2}(240 \mathrm{ml} \mathrm{CO} /$ day $)$ was continuously supplied to the reactor for sustaining the microbial growth ${ }^{41,55}$.

Table 4.1. Experimental conditions for the five operational phases in the continuous experiment

\begin{tabular}{llllll}
\hline \multirow{2}{*}{ Phases } & \multicolumn{5}{c}{ Variables used ( Changes are in bold font) } \\
& I & II & III & IV & V $^{1}$ \\
\hline Period (day) & $0-18$ & $19-25$ & $26-32$ & $33-39$ & $40-60$ \\
$\begin{array}{l}\text { Methanol feeding rate } \\
\text { (mMC/day) }\end{array}$ & 60 & $\mathbf{1 2 0}$ & $\mathbf{1 8 0}$ & $\mathbf{1 2 0}$ & 120 \\
$\begin{array}{l}\text { Butyrate feeding rate } \\
\text { (mMC/day) }\end{array}$ & - & - & - & - & $\mathbf{4 8 0}$
\end{tabular}

${ }^{1}$ Batch mode on day 47 due to clogging of the medium feeding tubes and its replacement.

The anaerobic bioreactor was inoculated with an undefined mixed culture from a bioreactor that performed methanol chain elongation in the previous study ${ }^{90}$. The synthetic medium 
consists of the basal medium, $200 \mathrm{mMC}$ of acetate and methanol and/or butyrate supplements in various concentration as indicated in Table 4.1. The composition of the basal medium was identical to the one used by the previous methanol chain elongation study ${ }^{90}$ (see also Support Information for Chapter 4; SI4). The synthetic medium was stored anaerobically in a refrigerator and replaced every week during the entire operational period.

\subsubsection{The batch test on the acidified supermarket waste (ASW) and methanol.}

A duplicate batch test on ASW and methanol was carried out using two serum bottles $($ volume $=250 \mathrm{ml})$. The serum bottles were filled with $150 \mathrm{ml}$ of ASW medium (see SI4 for the ASW preparation procedure including the hydrolysis/acidification and the heat shock applied) with methanol addition $(200 \mathrm{mM})$. The headspaces of both batch bottles $(100 \mathrm{ml})$ were vacuumed and flushed with a pure nitrogen gas for five times to remove the oxygen using a gas exchanger. After flushing with the pure nitrogen gas, the headspaces of both batch bottles were filled with a gas mixture containing $80 \% \mathrm{~N}_{2}$ and $20 \% \mathrm{CO}_{2}$ to 2 bars, which was also used in the previous methanol chain elongation experiment to sustain the growth of methanol chain-elongating microorganisms ${ }^{55,90}$. The batch bottles were incubated in a shaker. $\mathrm{pH}$ was not controlled during the entire incubation. The temperature was controlled at $35^{\circ} \mathrm{C}$, and the rotation speed was set at $120 \mathrm{rpm}$ for the entire batch experimental period (39 days).

\subsubsection{Sampling and Analyses}

For the continuous experiment, liquid samples $(1 \mathrm{ml})$ were taken from the anaerobic bioreactor 5 days per week and the synthetic medium every time it was prepared (approximately every week). Gas samples were collected from the headspace every two days. For the ASW batch experiments, liquid and gas samples were collected every four to five days. Headspace pressures of both batch bottles were measured with a gas pressure meter (GMH3150, Greisinger Electronics, Germany) before taking the liquid and gas samples. A liquid sample $(3 \mathrm{ml})$ was collected from each bottle immediately after the headspace pressure 
measurements. All liquid samples were measured using two Gas Chromatographs (GCs) with the standardised methods used in several previous studies ${ }^{32,90}$. The examined compounds include acetate, propionate, isobutyrate (2-Methylpropanoate), butyrate, isovalerate (3Methylbutanoate), valerate, isocaproate (4-Methylpentanoate), caproate, heptanoate, caprylate, methanol and ethanol in the liquid samples and $\mathrm{O}_{2}, \mathrm{~N}_{2}, \mathrm{CH}_{4}, \mathrm{H}_{2}$ and $\mathrm{CO}_{2}$ in the gas samples. The $\mathrm{pH}$ of all liquid samples was also measured. The actual consumption/production rate as presented in Figure 4.1, 4.2 and 4.3 was calculated based on the measured concentration of each organic compound. In other words, the production and consumption rate of an organic compound was calculate by subtracting its concentration in the growth medium from its concentration in the fermentation broth.

\subsection{Results}

\subsubsection{Continuous isobutyrate production from acetate and methanol with the presence of high butyrate concentration}

Increasing methanol feeding rate and the direct supply of butyrate to the bioreactor were the two strategies used for initiating isobutyrate formation in methanol chain elongation. Increasing methanol feeding rate from $60 \mathrm{mMC} /$ day to $120 \mathrm{mMC} /$ day in Phase II did increase the butyrate production rate from $55 \mathrm{mMC}$ /day to $102 \mathrm{mMC} /$ day on day 18 and 21 (Figure 4.1). Further increasing methanol feeding rate to $180 \mathrm{mMC}$ in Phase III resulted in an even higher butyrate production rate $(120 \mathrm{mMC} /$ day $)$. However, the higher butyrate production rate did not lead to the formation of isobutyrate. From day 28 to day 32, both butyrate production rate and the methanol consumption rate gradually decreased, indicating the inhibition of methanol chain elongation by the increased methanol feeding rate (Figure 4.1). On the other hand, the methane production rate increased along with the increased methanol feeding rate (Figure S4.2). In Phase IV, the methanol feeding rate was decreased to 120 $\mathrm{mMC} / \mathrm{day}$, which re-established the methanol chain elongation activity as the butyrate 
production rate in Phase IV was similar to that in Phase II. The methanol consumption in Phase IV was slightly less than such in Phase II. The less methanol consumption in Phase V compared with Phase II may be due to the more efficient use of methanol by the shaped reactor microbiome.

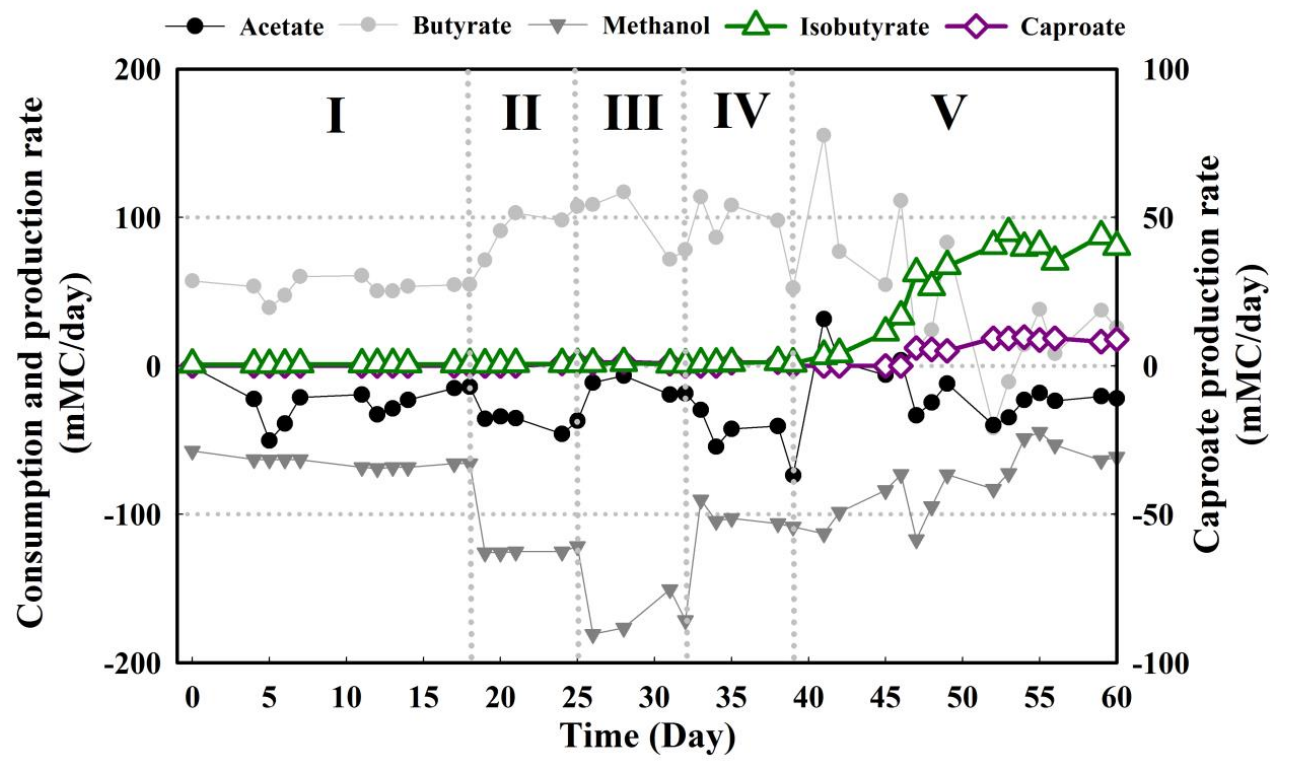

Figure 4.1. Product formation and substrate consumption rates (in millimolar carbon per day) over the entire operational period of the continuous methanol chain elongation. Negative values indicate the overall consumption of the substrate, and positive values indicate the overall production of the compound. The Roman numerals refer to different operational conditions (see Table 4.1). Methanol and acetate were supplied during the entire experiment, and butyrate was supplied in Phase V as an additional substrate.

In Phase $\mathrm{V}$, when butyrate ( $480 \mathrm{mMC} /$ day) was directly fed to the bioreactor and Vitamin B12 supply was increased, the isobutyrate production started (Figure 4.1). From day 41, the isobutyrate production rate gradually increased and lasted for 20 days until the end of the experiment. Meanwhile, the butyrate production rate gradually decreased. From day 50 


\section{Chapter 4}

onwards, isobutyrate production rate exceeded the butyrate production rate. The highest isobutyrate production rate achieved was around $90 \mathrm{mMC} / \mathrm{day}(2.0 \mathrm{~g} / \mathrm{L} / \mathrm{day})$ with the isobutyrate concentration up to $136 \mathrm{mMC}(3.0 \mathrm{~g} / \mathrm{L})$ in the reactor broth on day 53 . In the operational period from day 50 to 60 , isobutyrate remained as the most dominant product (hereinafter referred this period as the isobutyrate-dominant period). Other products formed in the same period were caproate, butyrate and methane. The average isobutyrate production rate in the isobutyrate-dominant period was $81 \pm 6 \mathrm{mMC}$ /day (Figure $4.2 \mathrm{a}$ ), which was the highest among all the products. The carbon recovery in this period was mostly higher than 90\% (Figure S4.3). The unrecovered carbon could be most likely attributed to the biomass formation and/or the unconverted yeast extract. The $\mathrm{pH}$ of the fermentation broth in this period ranged from 6.2 to 6.5 (Figure S4.4) without any external pH control.

(a)

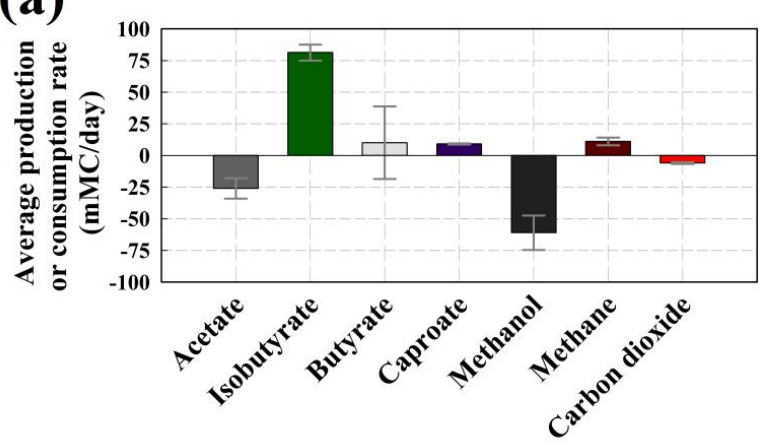

(b)

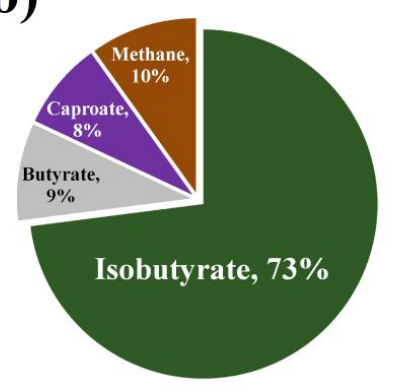

Figure 4.2. Overview of (a) the average product formation and substrate consumption rates and (b) product distribution in carbon in the isobutyrate-dominant period (including all data points from day 50 to day 60). The error bars represent the standard deviations of the production/consumption rates.

In the isobutyrate-dominant period (day 50-60), acetate and methanol were the main substrates for the isobutyrate formed. They were the primarily consumed substrates among all the substrates supplied (Figure 4.2a). As neither of acetate and methanol could solely provide 
sufficient carbon for the amount of isobutyrate formed, both acetate and methanol must have acted as the substrates for the isobutyrate formation. In this case, methanol was the electron donor while acetate the electron acceptor. However, the amount of carbon in the overall methanol and acetate consumption is higher than the amount of carbon in the produced isobutyrate, which indicates that part of the methanol and acetate might be converted to other products besides isobutyate, e.g. methane, caproate and butyrate. Butyrate was also supplied in the isobutyrate-dominant period as a substrate for methanol chain elongation, which was another potential substrate for the isobutyrate formation. Interestingly, in the isobutyratedominant period, butyrate had mostly a gross production (except on day 52 and 53) instead of a gross consumption even with such high butyrate feeding rate (Figure 4.1). This newly produced butyrate possibly resulted from methanol chain elongation on methanol and acetate $^{90}$. In this case, it is most likely that the bio-isomerisation of butyrate into isobutyrate and methanol chain elongation of acetate and methanol into butyrate occurred concurrently; the latter had a higher production rate, so a gross butyrate production was observed in Phase $\mathrm{V}$. The other potential substrates for the isobutyrate formation include $\mathrm{CO}_{2}$ and yeast extract. $\mathrm{CO}_{2}$ was less likely a substrate for the isobutyrate formation as the $\mathrm{CO}_{2}$ consumption in the isobutyrate-dominant period was not significant and was similar to such in the other operational periods (Phase I-IV) where isobutyrate production was not observed (Figure S4.2). Yeast extract was unlikely one of the main substrate for the isobutyrate production because it could supply only a minimal amount of carbon (see SI4) and also it did not lead to isobutyrate formation under similar conditions ${ }^{90}$.

As for the product distribution, isobutyrate accounted for $73 \%$ of the total carbon in all the products in the isobutyrate-dominant period (Figure $4.2 \mathrm{~b}$ ), while $10 \%$ attributed to the methane formation. This showed that even though a methanogenic inhibitor was not used in the continuous experiment, methanogenesis was not able to outcompete methanol chain 
elongation. Butyrate and caproate were minor products that accounted for $9 \%$ and $8 \%$, respectively. Nevertheless, the total carbon in the produced isobutyrate accounted only for $12 \%$ of the total carbon input (data not shown). This was due to a large proportion of supplied substrates that remained unconverted ( $83 \%$ of the total carbon input), i.e. butyrate, acetate and methanol. As this is the first demonstration of the proof-of-principle of isobutyrate formation using acetate and methanol as the substrates, further process optimisation is necessary to increase the substrate conversion efficiency.

\subsubsection{Continuous caproate production from acetate and methanol}

Although producing caproate is not the main objective of the present study, the continuous caproate production from acetate and methanol using a mixed culture is reported for the first time (Figure 4.1). In the isobutyrate-dominant period, a relatively stable caproate $(9 \pm 1$ $\mathrm{mMC} /$ day) production was observed along with the simultaneous isobutyrate and butyrate production, which lasted for 10 days. The observed caproate could be produced from methanol and acetate or methanol and butyrate via methanol chain elongation, as other potential electron donors, e.g. $\mathrm{H}_{2}$ and ethanol, were not detected during the entire experimental period. Caproate formation from methanol and acetate ${ }^{55}$ as well as from methanol and butyrate ${ }^{47}$ by a pure culture of Eubacterium limosum was reported. Previously we also demonstrated the caproate production from acetate and methanol with a mixed culture but only in a batch operation ${ }^{90}$. In the present study, we achieved the continuous caproate production via methanol chain elongation under a non-sterile condition.

\subsubsection{Biosynthesis of isobutyrate using a real organic waste and methanol}

Isobutyrate production using a real organic waste, i.e. acidified supermarket waste (ASW), as a feedstock was demonstrated for the first time in the present study (Figure 4.3a and Figure 4.4). The ASW used in the present study contained a large amount of SCFAs especially butyrate $(>400 \mathrm{mMC})$. After the methanol addition and the 39-day batch incubation, 
isobutyrate was the most dominant product $(31.7 \pm 0.6 \mathrm{mMC})$ that accounted for $63 \%$ of the total carbon in all the products (Figure 4.3b; see also Figure 4.4 for the concentration profile). Valerate and caproate were the by-products. Trace amounts of acetate and isovalerate were also produced. The main consumed organic acids were butyrate and propionate. Other consumed substrates included methanol that was added and ethanol that was produced in-situ during the acidification of the supermarket waste. $\mathrm{CO}_{2}$ (around 1 millimole in total) added in the headspace was completely consumed at the end of the experiment. In the ASW medium, there might be larger soluble organic matters (e.g. amino acids) that were not monitored, which might have also contributed to a part of the product formation during the batch test. However, based on the overall carbon recovery efficiency $(97 \%$, calculated from all the substances measured), the amount of product formation resulted from the degradation of these bigger soluble organic matters could not be significant. The unrecovered carbon (3\%) could be due to biomass formation.

(a)

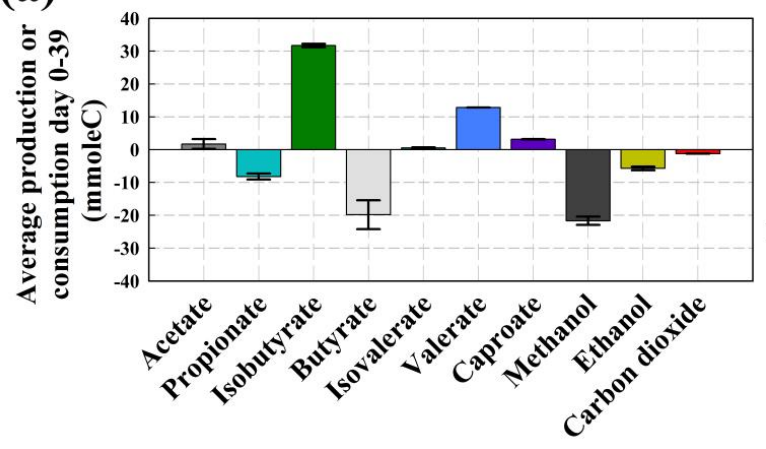

(b)

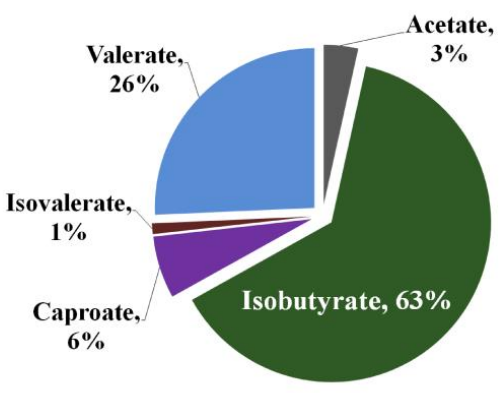

Figure 4.3. Overview of (a) the average product formation and substrate consumption (in millimole carbon) and (b) product distribution in carbon in the entire batch test (including all data points from day 0 to day 39) on ASW and methanol. Methanol was externally added as an additional substrate, and the other consumed organic acids were originated from the ASW. The error bars indicated the maximal and minimum values derived from the duplicates. 


\section{Chapter 4}

The isobutyrate formed was evidently produced from butyrate and methanol based on the production and consumption profile in Figure 4.3 and the concentration profile in Figure 4.4. As aforementioned, possibly butyrate continuously transformed into isobutyrate via the bioisomerisation $^{128,131,134,135}$ and simultaneously methanol chain elongation kept producing butyrate from methanol and/or acetate ${ }^{90}$. Valerate most likely formed via the chain elongation of propionate and ethanol, a known process that could occur with a mixed culture ${ }^{71}$. In a chain elongation process containing ethanol, acetate is produced through ethanol oxidation for yielding ATP to support the microbial growth ${ }^{41}$, which may explain a part of the gross acetate production observed (see also the later discussion section for a further elaboration on the acetate production in the batch test). However, valerate production from methanol chain elongation of propionate was also reported in a previous study ${ }^{47}$. It could not be excluded that part of the valerate was synthesised from methanol and propionate. Although it was not possible to quantify the extent of ethanol and methanol chain elongation for the formation of valerate in the batch test, supplying multiple electron donors (methanol and ethanol in this batch test) in chain elongation was shown to be interesting due to the simultaneous formation of multiple useful platform chemicals (e.g. isobutyrate, isovalerate, valerate and caproate in this batch test). Moreover, the result from the batch test demonstrates the feasibility of isobutyrate biosynthesis using a real organic residue, which is never reported before and represents a novel renewable process for valorising organic residues into platform chemicals. 


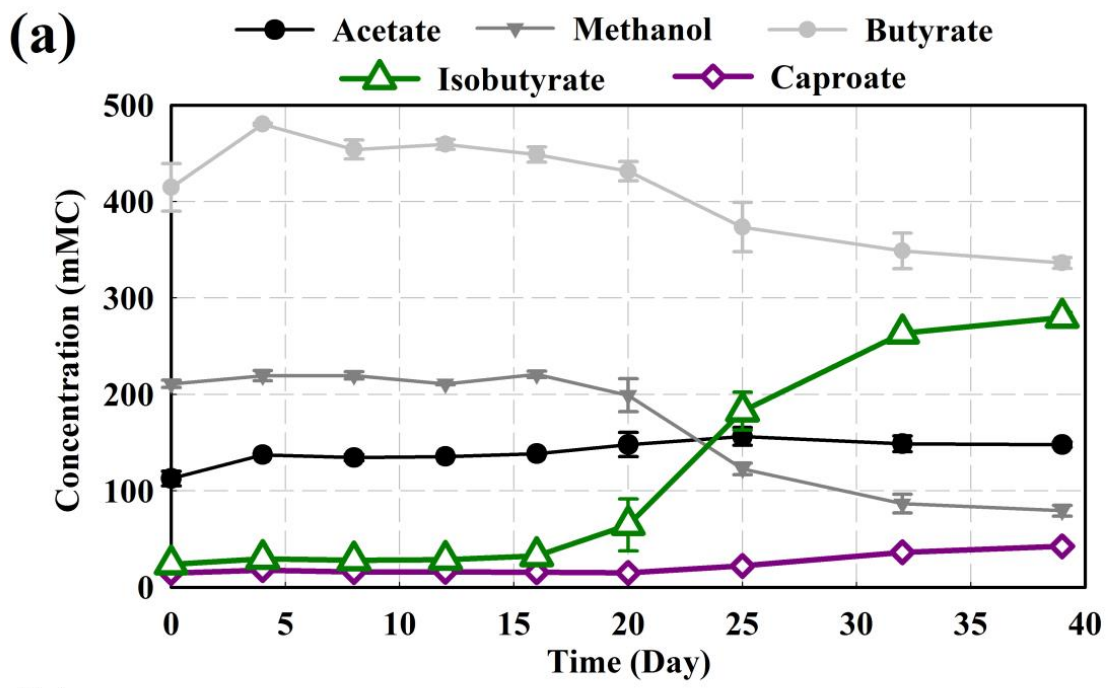

(b)

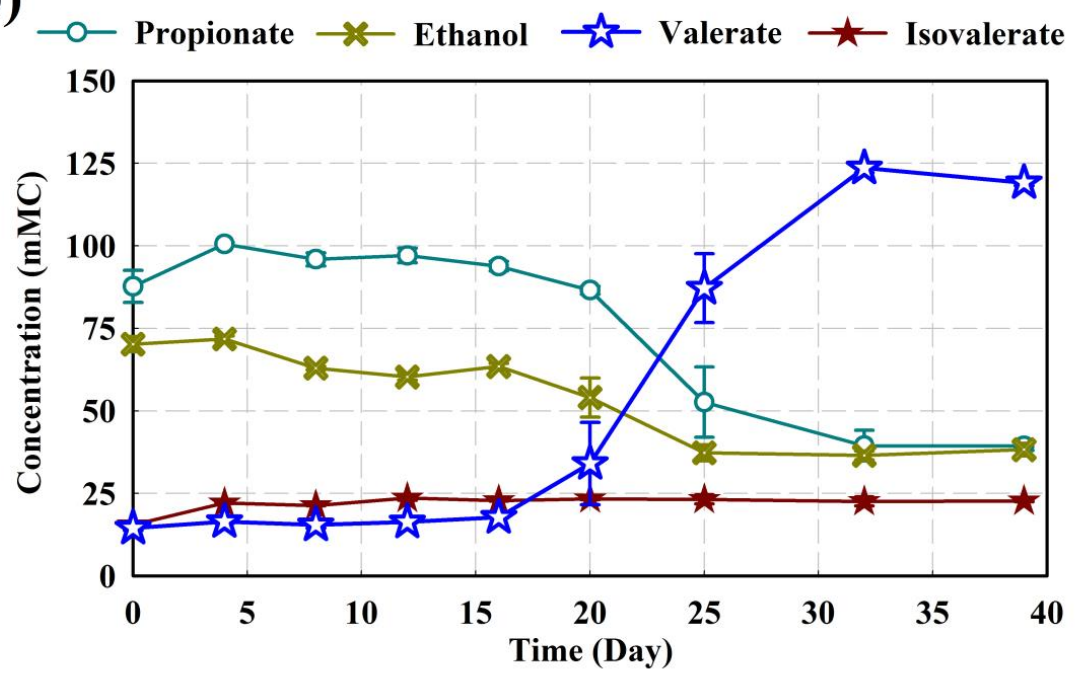

Figure 4.4. Concentration profile of all organic acids and alcohol monitored during the entire batch test on ASW except for isocaproate, heptanoate and capryalte that were always under detection limit. To improve the readability of the figure all substances were classified into two categories, the substances possibly involved in methanol chain elongation (a) and in ethanol chain elongation (b). The error bars indicate the minimal and maximal value based on the duplicate. 


\subsection{Discussion}

4.4.1. The proposed mechanism and the essential conditions for isobutyrate biosynthesis via methanol chain elongation

A direct biological pathway for isobutyrate formation from acetate and methanol has never been reported to authors' current knowledge. The isobutyrate formation in the present study was most likely resulted from the concurrent bio-isomerisation (reaction 1 in Table 4.2; also refer to Figure 4.5) and methanol chain elongation (reaction 5.1-5.3 in Table 4.2), as proposed in the introduction. Herein we provide a more in-depth explanation and an elaborated discussion on our hypothesis based on the methanogenic anaerobic digestion as illustrated in Figure 4.5 (see also Table 4.2 for the example reactions of all the biological reactions involved).

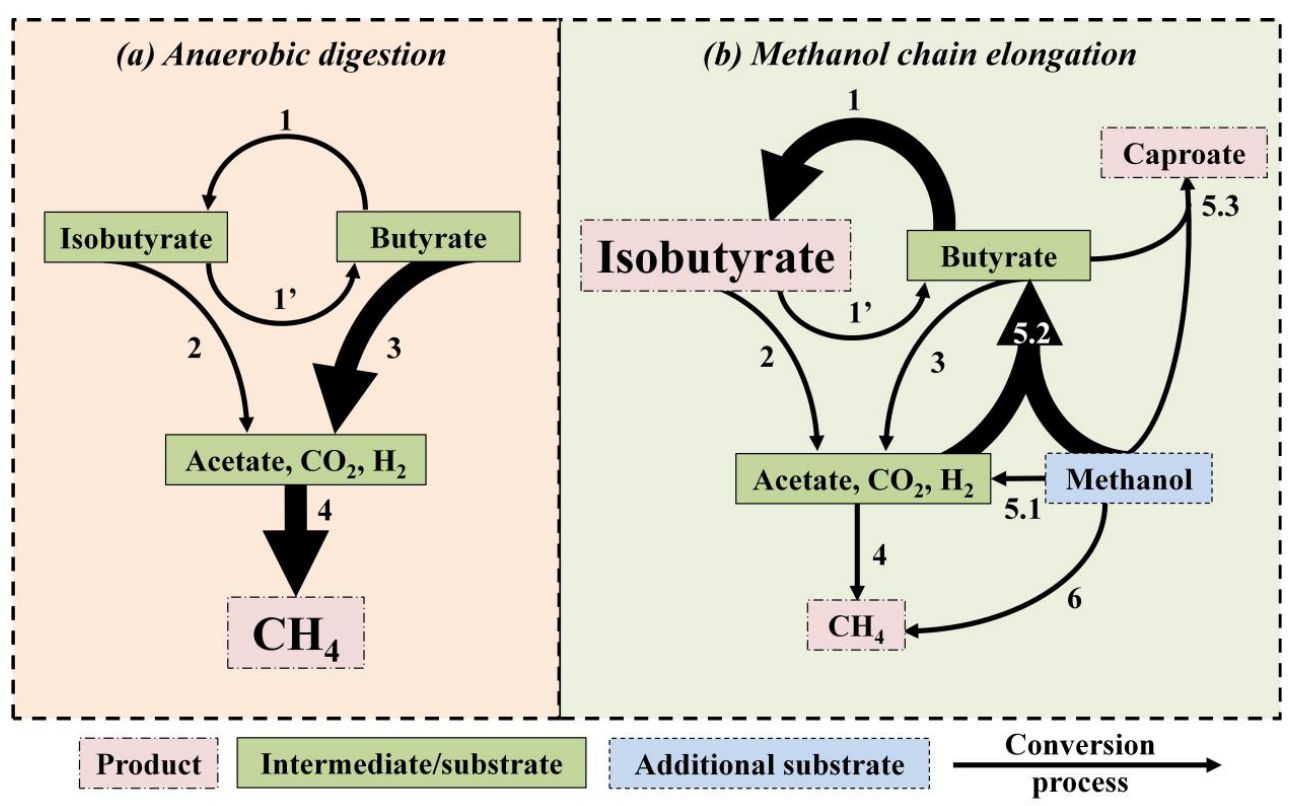

Figure 4.5. Overview of the conversion routes in a methanogenic anaerobic digestion system (a) and a methanol chain elongation system (b), which is used to explain the isobutyrate formation observed in the present study. A thick arrow indicates the dominant pathway. 
Table 4.2. List of example reactions of all the conversion routes in Figure 4.5.

\begin{tabular}{|c|c|c|}
\hline & Biochemical conversion process & $\Delta \mathrm{G}_{\mathrm{r}}^{\mathrm{o}^{\prime}}(\mathrm{kJ} /$ reaction $)$ \\
\hline \multirow[t]{2}{*}{1} & Isomerisation of butyrate into isobutyrate ${ }^{133}$ & 0 \\
\hline & $\mathrm{CH}_{3}\left(\mathrm{CH}_{2}\right)_{2} \mathrm{COO}^{-} \rightarrow\left(\mathrm{CH}_{3}\right)_{2} \mathrm{CHCOO}^{-}$ & \\
\hline \multirow[t]{2}{*}{1 ' } & De-isomerisation of isobutyrate into butyrate ${ }^{133}$ & 0 \\
\hline & $\left(\mathrm{CH}_{3}\right)_{2} \mathrm{CHCOO}^{-} \rightarrow \mathrm{CH}_{3}\left(\mathrm{CH}_{2}\right)_{2} \mathrm{COO}^{-}$ & \\
\hline \multirow[t]{2}{*}{2} & Direct degradation of isobutyrate to acetate, $\mathrm{CO}_{2}$ and $\mathrm{H}_{2}{ }^{139}$ & 152.8 \\
\hline & $\left(\mathrm{CH}_{3}\right)_{2} \mathrm{CHCOO}^{-}+6 \mathrm{H}_{2} \mathrm{O} \rightarrow \mathrm{CH}_{3} \mathrm{COO}^{-}+2 \mathrm{HCO}_{3}^{-}+6 \mathrm{H}_{2}+2 \mathrm{H}^{+}$ & \\
\hline \multirow[t]{2}{*}{3} & Butyrate degradation to acetate and $\mathrm{H}_{2}{ }^{133}$ & 48.3 \\
\hline & $\mathrm{CH}_{3}\left(\mathrm{CH}_{2}\right)_{2} \mathrm{COO}^{-}+2 \mathrm{H}_{2} \mathrm{O} \rightarrow 2 \mathrm{CH}_{3} \mathrm{COO}^{-}+2 \mathrm{H}_{2}+\mathrm{H}^{+}$ & \\
\hline \multirow[t]{4}{*}{4} & Methane production via acetoclastic methanogenesis ${ }^{26}$ & -31.0 \\
\hline & $\mathrm{CH}_{3} \mathrm{COO}^{-}+\mathrm{H}_{2} \mathrm{O} \rightarrow \mathrm{CH}_{4}+\mathrm{HCO}_{3}^{-}$ & \\
\hline & Methane production via hydrogenotrophic methanogenesis ${ }^{133}$ & -135.6 \\
\hline & $4 \mathrm{H}_{2}+\mathrm{HCO}_{3}^{-}+\mathrm{H}^{+} \rightarrow \mathrm{CH}_{4}+3 \mathrm{H}_{2} \mathrm{O}$ & \\
\hline \multirow[t]{2}{*}{5.1} & Methanol chain elongation to acetate ${ }^{112}$ & -221.6 \\
\hline & $4 \mathrm{CH}_{3} \mathrm{OH}+2 \mathrm{HCO}_{3}^{-} \rightarrow 3 \mathrm{CH}_{3} \mathrm{COO}^{-}+\mathrm{H}^{+}+4 \mathrm{H}_{2} \mathrm{O}$ & \\
\hline \multirow[t]{2}{*}{5.2} & Methanol chain elongation to butyrate ${ }^{112}$ & -541.9 \\
\hline & $10 \mathrm{CH}_{3} \mathrm{OH}+2 \mathrm{HCO}_{3}^{-} \rightarrow 3 \mathrm{CH}_{3}\left(\mathrm{CH}_{2}\right)_{2} \mathrm{COO}^{-}+\mathrm{H}^{+}+10 \mathrm{H}_{2} \mathrm{O}$ & \\
\hline \multirow[t]{2}{*}{5.3} & Methanol chain elongation to caproate (hypothetical) & -106.9 \\
\hline & $2 \mathrm{CH}_{3} \mathrm{OH}+\mathrm{CH}_{3}\left(\mathrm{CH}_{2}\right)_{2} \mathrm{COO}^{-} \rightarrow \mathrm{CH}_{3}\left(\mathrm{CH}_{2}\right)_{4} \mathrm{COO}^{-}+2 \mathrm{H}_{2} \mathrm{O}$ & \\
\hline \multirow[t]{2}{*}{6} & Methane production via methylotrophic methanogenesis ${ }^{140}$ & -314.6 \\
\hline & $4 \mathrm{CH}_{3} \mathrm{OH} \rightarrow 3 \mathrm{CH}_{4}+\mathrm{HCO}_{3}^{-}+\mathrm{H}^{+}+\mathrm{H}_{2} \mathrm{O}$ & \\
\hline
\end{tabular}

In a methanogenic anaerobic digestion system (Figure 4.5a), butyrate is a common intermediate product that is degraded via two pathways, the $\beta$-oxidation into acetate and hydrogen (reaction 3) or bio-isomerisation of butyrate into isobutyrate that is further degraded into acetate, $\mathrm{CO}_{2}$ and $\mathrm{H}_{2}$ coupled with sulfate reduction (reaction 2) ) $^{129,135,139,141}$. 


\section{Chapter 4}

The produced acetate, $\mathrm{H}_{2}$ and/or $\mathrm{CO}_{2}$ are further converted via acetoclastic or hydrogenotrophic methanogenesis into $\mathrm{CH}_{4}$ and $\mathrm{CO}_{2}$ (reaction 4). The $\beta$-oxidation of butyrate is usually the dominant butyrate-degrading pathway in a methanogenic anaerobic digestion system (Figure 4.5a). In a methanol chain elongation system, the methanol addition stimulates butyrate formation from methanol, $\mathrm{CO}_{2}$ and/or acetate (reaction 5$)^{47,55,90}$, which can outcompete the $\beta$-oxidation of butyrate and resulted in an accumulation of butyrate ${ }^{90}$. The continuous accumulation of butyrate could stimulate bio-isomerisation of butyrate into isobutyrate (reaction 1). This was also observed in several previous studies especially when methanogenic activity (reaction 4) is not effective in degrading the accumulated organic acids due to the presence of a methanogenic inhibitor ${ }^{128,131,134,135}$ (Figure 4.5b). In both experiments in the present study, the methanogenic activity might indeed have been suppressed by the high organic acid concentrations, especially by the high butyrate concentration $^{136}$. Butyrate accumulation clearly plays an important role in the isobutyrate formation observed in the present study. In the continuous experiment, the butyrate accumulated in-situ was not sufficient to stimulate isobutyrate formation, and an additionally supplied butyrate was required. On the other hand, the butyrate concentration in the ASW was already sufficient to trigger the bio-isomerisation. Future study may try to use ASW and methanol directly for demonstrating the continuous isobutyrate biosynthesis.

Despite that bio-isomerisation of butyrate into isobutyrate has been reported in many previous studies $128,131,132,134,135$, the ecological function of such process remains to be revealed. As the bio-isomerisation alone does not provide any growth energy, it has to be coupled to other reactions that are of survival value. In a methanol chain elongation system, a possible function of the bio-isomerisation could be to detoxify the inhibitory effect of the unbranched SCFAs, especially the butyrate. A known methanol chain elongator, i.e. E.limosum, was reported to be inhibited by elevated butyrate concentrations ${ }^{142}$. Lindley et al. 
(1987) showed that when butyrate concentration is lower than $800 \mathrm{mMC}$, E.limosum can still produce butyrate from methanol and $\mathrm{CO}_{2}$. When butyrate concentration is higher than 800 $\mathrm{mMC}$, E.limosum starts to convert butyrate to other products including acetate, caproate and an unknown compound that is suspected to be an extracellular polymer ${ }^{47}$. It is possible that converting butyrate into other organic acids, e.g. caproate, the unknown compound or even isobutyrate in the present study, could be a strategy for microorganisms to overcome the butyrate inhibition. In this case, an electron donor that continuously stimulates the butyrate formation is essential to push the bio-isomerisation of butyrate continuously. Methanol and ethanol are both electron donors that can stimulate the butyrate formation under a non-sterile condition $^{32,37,90}$. Nevertheless, using ethanol as the electron donor mostly results in the formation of caproate as the dominant product instead of butyrate ${ }^{39,66}$. In contrast, using methanol as the electron donor leads to the formation of butyrate as the dominant product, which stimulates the isobutyrate formation.

In addition to the suppression of methanogenic activity, the accumulation of butyrate and the presence of an electron donor, there are other potential key factors for stimulating bioisomerisation that remain to be further investigated, e.g. $\mathrm{pH}$, Vitamin $\mathrm{B} 12$ concentration, the composition of the shaped reactor microbiome, etc. Increasing $\mathrm{pH}$ to 6.5 in methanol chain elongation was shown to increase the butyrate accumulation but also the methanogenic $\operatorname{activity}^{90}$. On the other hand, decreasing $\mathrm{pH}$ may be effective in further inhibiting methanogens. Vitamin B12 is an essential catalyst for both methanol chain elongation and bio-isomerisation ${ }^{133,138,143}$. In Phase V of the continuous experiment, Vitamin B12 was increased to ensure the sufficient availability of the catalyst. Although a preliminary test showed that supplying butyrate without increasing Vitamin B12 concentration still triggered the isobutyrate formation in methanol chain elongation (data not shown), it remains unclear 


\section{Chapter 4}

whether a higher Vitamin B12 concentration could further stimulate bio-isomerisation. These factors should be further investigated in the future research.

\subsubsection{The effect of SCFA supplementation and concentrations on isobutyrate formation}

SCFAs are essential substrates for isobutyrate biosynthesis via methanol chain elongation, and different SCFA concentrations lead to different substrate utilisation patterns. In the continuous experiment, acetate was the main consumed substrate while butyrate was mostly produced along with the isobutyrate formation (Figure 4.1). In contrast, in the batch test, net production of acetate was observed (Figure 4.3), and butyrate was likely the main substrate consumed for the isobutyrate formation. Such difference in the substrate utilisation may be explained by using E.limosum as a model microorganism. E.limosum was the only reported bacteria that produces caproate from methanol, acetate, $\mathrm{CO}_{2}$ and/or butyrate ${ }^{47,}{ }^{55}$. Possibly E.limosum or a similar microorganism was responsible for the butyrate and even the caproate formation via methanol chain elongation in the present study. It was reported that when the acetate concentration was below $190 \mathrm{mMC}(95 \mathrm{mM})$, methanol and $\mathrm{CO}_{2}$ were converted by a pure culture E.limosum into both acetate and butyrate as the metabolites ${ }^{117}$. The same study reported that, with an acetate concentration higher than $280 \mathrm{mMC}$, E.limosum started to consume acetate and produce butyrate as the only metabolite. Thus, the acetate concentration does affect the metabolism of E.limosum. In the continuous experiment on the synthetic medium, $200 \mathrm{mMC}$ acetate was continuously supplied to keep sufficient acetate concentration, while in the batch test on ASW the initial acetate concentration was around $113 \mathrm{mMC}$ resulted from the acidification of the supermarket waste (Figure 4.4). When acetate concentration is low (i.e. $<190 \mathrm{mMC}$ ), part of the methanol supplied may end up in the formation of extra acetate, which is a less desired product compared with butyrate and isobutyrate. Given this context, we anticipate that if none of the SCFAs are supplied in 
methanol chain elongation, methanol will be elongate first to acetate and butyrate, and isobutyrate may be produced only when acetate and butyrate accumulation reached certain concentrations. Therefore, supplying sufficient SCFAs from a cheap source like ASW is essential, and steering the acidification process of ASW to produce sufficient acetate is beneficial in preventing the unnecessary acetate formation from methanol in methanol chain elongation.

Table 4.3. Overview of the currently reported isobutyrate production via microbial processes.

\begin{tabular}{|c|c|c|c|c|}
\hline Source & Substrate & Reactor type & Biocatalyst & $\begin{array}{l}\text { Isobutyrate concentration } \\
\text { (conc.), rate and yield }{ }^{\dagger}\end{array}$ \\
\hline $\begin{array}{l}\text { Zhang et } \\
\text { al. } \\
(2011)^{57}\end{array}$ & $\begin{array}{l}\text { Glucose- } 40 \mathrm{~g} / \mathrm{L} \\
\text { Yeast extract- } 5 \\
\mathrm{~g} / \mathrm{L}^{*}\end{array}$ & $\begin{array}{l}\text { Batch flask } \\
\text { with } 5 \mathrm{ml} \\
\text { working } \\
\text { volume. }\end{array}$ & $\begin{array}{l}\text { Engineered E.coli } \\
\text { strain BW25113 }\end{array}$ & $\begin{array}{l}\text { Conc. }=11.7 \mathrm{~g} / \mathrm{L} \\
\text { Yield }=0.27 \mathrm{~g} / \mathrm{gCOD}_{\mathrm{S}}\end{array}$ \\
\hline $\begin{array}{l}\text { Lang et } \\
\text { al. } \\
(2014)^{122}\end{array}$ & $\begin{array}{l}\text { Glucose- } 20 \mathrm{~g} / \mathrm{L} \\
\text { Yeast extract- } 5 \\
\mathrm{~g} / \mathrm{L}\end{array}$ & $\begin{array}{l}\text { Batch flask } \\
\text { with } 250 \mathrm{ml} \\
\text { working } \\
\text { volume. }\end{array}$ & $\begin{array}{l}\text { Engineered } \\
\text { Pseudomonas sp. } \\
\text { Strain VLB120 }\end{array}$ & $\begin{array}{l}\text { Conc } .=2.0 \mathrm{~g} / \mathrm{L} \\
\text { Yield }=0.11 \mathrm{~g} / \mathrm{gCOD}_{\mathrm{S}}\end{array}$ \\
\hline $\begin{array}{l}\text { This study } \\
\text { (2016) }\end{array}$ & $\begin{array}{l}\text { Acidified } \\
\text { supermarket } \\
\text { waste } \\
\text { Methanol- } 6.9 \\
\text { g/L }\end{array}$ & $\begin{array}{l}\text { Batch flask } \\
\text { with } 150 \mathrm{ml} \\
\text { working } \\
\text { volume. }\end{array}$ & $\begin{array}{l}\text { Undefined mixed } \\
\text { culture microbiome } \\
\text { within the substrate. }\end{array}$ & $\begin{array}{l}\text { On Day } 39, \\
\text { Conc. }=6.2 \mathrm{~g} / \mathrm{L} \\
\text { Yield }=0.15 \mathrm{~g} / \mathrm{gCOD}_{\mathrm{S}}\end{array}$ \\
\hline $\begin{array}{l}\text { This study } \\
\text { (2016) }\end{array}$ & $\begin{array}{l}\text { Acetate- } 5.5 \mathrm{~g} / \mathrm{L} \\
\text { Butyrate- } 15.5 \\
\mathrm{~g} / \mathrm{L} \\
\text { Methanol- } 7.0 \\
\mathrm{~g} / \mathrm{L} \\
\text { Yeast extract- } \\
1.0 \mathrm{~g} / \mathrm{L}\end{array}$ & $\begin{array}{l}\text { 1L Anaerobic } \\
\text { Bioreactor, } \\
\text { continuous } \\
\text { operation. }\end{array}$ & $\begin{array}{l}\text { Undefined mixed } \\
\text { culture microbiome } \\
\text { from a previous } \\
\text { methanol chain } \\
\text { elongation study. }\end{array}$ & $\begin{array}{l}\text { On Day 53: } \\
\text { Rate }=2.0 \mathrm{~g} / \mathrm{L} / \text { day } \\
\text { Conc. }=3.0 \mathrm{~g} / \mathrm{L} \\
\text { Yield }=0.06 \mathrm{~g} / \mathrm{gCOD}_{\mathrm{S}}\end{array}$ \\
\hline $\begin{array}{l}\text { The yic } \\
\text { was not in } \\
\text { reviewed }\end{array}$ & $\begin{array}{l}\text { vas evaluated } \\
\text { led in the total } \\
\text { ies. }{ }^{*} \text { Recalcula }\end{array}$ & $\begin{array}{l}\text { d on the tot } \\
\text { strate as the } \\
\text { from Zhang }\end{array}$ & $\begin{array}{l}\text { OD of the substrat } \\
\text { ct conversion of wh } \\
\text { l. }(2011)^{57} \text {. }\end{array}$ & $\begin{array}{l}\text { gCODs). The yeast extract } \\
\text { was not reported in all the }\end{array}$ \\
\hline
\end{tabular}




\subsubsection{Comparison with the existing isobutyrate biosynthesis processes}

Isobutyrate production via methanol chain elongation uses a cheap and renewable feedstock and conversion process, but its performance is still comparable to the existing isobutyrate biosynthesis processes. There are currently two reported isobutyrate biosynthesis processes, but both of which use glucose as the substrate and an engineered microbe as the biocatalyst (Table 4.3$)^{57,122}$. Glucose is mostly derived from crop-based feedstocks, which may again raise the food versus fuel competition ${ }^{13}$. In contrast, in the present study, the feedstock, i.e. SCFAs and methanol, can be derived from residual organic materials that are cheap (owing to the negative economic value), renewable and widely available. Moreover, a self-regenerating biocatalyst that can work in a non-sterile condition is used, which can avoid the energy-intensive sterilisation process ${ }^{20}$. As for the production performance, both of the isobutyrate concentration and the yield achieved in the batch test on ASW are in the range of such achieved by the existing processes (Table 4.3). Moreover, in the present study, valueadded products like valerate and caproate were not yet accounted in the product yield. When including the other products in the calculation, the overall yield will of course increase. However, it should be noted that the presence of other products may also decrease the effectiveness of the downstream process, i.e. separation and purification. As for the production rate, the comparison cannot be made as the present study is the first and the only continuous isobutyrate biosynthesis reported. Overall, the use of a cheap and waste-based feedstock makes methanol chain elongation a competitive and promising isobutyrate production process; the fact that it can be continuously operated further enhances the applicability of the process. Future studies may focus on revealing the microbial pathways behind the isobutyrate biosynthesis, testing the feasibility of using real waste feedstocks in the continuous operation, examining the stability of a long-term operation as such done for ethanol chain elongation process ${ }^{66}$, increasing the product specificity of isobutyrate and exploring the proper downstream processes. 


\subsection{Conclusion}

In the present study, we demonstrated the proof-of-principle for isobutyrate production from organic waste via methanol chain elongation. Continuous isobutyrate production (2.0 $\mathrm{g} / \mathrm{L} /$ day) was shown to be feasible using a synthetic medium containing methanol, acetate and butyrate. Use a real waste stream, i.e. supermarket waste, for isobutyrate production via methanol chain elongation was also demonstrated in a batch test. In the batch test, $63 \%$ of all identified products were allocated to isobutyrate at the highest achieved concentration of 6.2 $\mathrm{g} / \mathrm{L}$. The use of a renewable waste feedstock and a self-regenerating non-hazardous biocatalyst makes isobutyrate biosynthesis via methanol chain elongation a potentially more sustainable alternative compared with the current isobutyrate production process. 


\section{Support Information for Chapter 4 (SI4)}

\section{Estimation of the carbon from the conversion of yeast extract.}

The carbon contribution of the yeast extract to the product formation in the continuous methanol chain elongation on the synthetic medium was estimated. A composition of yeast extract, $\mathrm{CH}_{1.7} \mathrm{O}_{0.5} \mathrm{~N}_{0.2}$, was derived from a previous study and used in this study to calculate the initially available carbon from the yeast extract added ${ }^{114}$. Based on this estimation and the concentration of the yeast extract used $(1 \mathrm{~g} / \mathrm{L}), 34.5 \mathrm{mMC}$ was available assuming that the yeast extract added was completely metabolised, which was around $20.7 \mathrm{mMC}$ /day in terms of loading rate based on the HRT.

\section{Composition of the basal medium.}

The basal medium contained $\mathrm{NH}_{4} \mathrm{H}_{2} \mathrm{PO}_{4}-3.6 \mathrm{~g} / \mathrm{L}, \mathrm{MgCl}_{2} .6 \mathrm{H}_{2} \mathrm{O}-0.33 \mathrm{~g} / \mathrm{L}, \mathrm{MgSO}_{4} .7 \mathrm{H}_{2} \mathrm{O}$ $-0.2 \mathrm{~g} / \mathrm{L}, \mathrm{CaCl}_{2} .2 \mathrm{H}_{2} \mathrm{O}-0.2 \mathrm{~g} / \mathrm{L}, \mathrm{KCl}-0.15 \mathrm{~g} / \mathrm{L}$, yeast extract $-1 \mathrm{~g} / \mathrm{L}$, Vitamin solution 1 $\mathrm{ml} / \mathrm{L}$ and trace element solution $1 \mathrm{ml} / \mathrm{L}^{113}$. Vitamin B12 was increased to $400 \mu \mathrm{g} / \mathrm{L}$ in Phase $\mathrm{V}$ to ensure the sufficient availability of the essential coenzyme required for catalysing the isomerisation of butyrate ${ }^{134}$.

\section{Preparation of the acidified supermarket waste (ASW)}

The ASW was prepared by acidifying a supermarket waste with a mixed culture microbiome as the biocatalyst. The supermarket waste was collected from ROTIE (Lijnden, The Netherlands), a supermarket waste processing company in the Netherlands. $10 \mathrm{~kg}$ of supermarket waste consisting of mainly outdated food scraps was collected. The collected supermarket waste was already shredded into a slurry-like solution, which had a volatile solid content approximately $15 \mathrm{wt} \% .10 \mathrm{~kg}$ of tap water was added to the supermarket waste to dilute the volatile solid content to approximately $7.5 \mathrm{wt} \%$. $\mathrm{NaOH}(5 \mathrm{M})$ was added to adjust the $\mathrm{pH}$ of the diluted supermarket waste solution to 6.8. A mixed culture inoculum (around 
$0.4 \mathrm{~kg}$ ) from an acidification bioreactor (ChainCraft, the Netherlands) was added to enhance the acidification.

A 20-litre batch reactor was used to perform the acidification of the supermarket waste. The reactor was continuously stirred at 44rpm (Haarlem Holland motor, type TD315, the Netherlands). A water jacket and a heating bath were installed to control the reactor at $35^{\circ} \mathrm{C}$ (Julabo F25 \& Julabo HD, Germany). The pH in the reactors was monitored (Liquisys M, Endress \& Hauser) and controlled at around 5.9 \pm 0.4 using a $\mathrm{pH}$ controller with a $5 \mathrm{M} \mathrm{NaOH}$ solution. No gas was supplied during the acidification process. Gas meter (Schlumberger, USA) was installed to monitored the gas production during the acidification of the supermarket waste. $2 \mathrm{ml}$ of antifoam was added before the start of the acidification.

The acidification process lasted 14 days. After the acidification, the effluent containing ASW was centrifuged (5000 rpm for 10 minutes) and sieved to remove floating particles to derive the ASW ready for further experiments. In the present study, the ASW was further treated by applying a heat shock $\left(100^{\circ} \mathrm{C}\right.$ for 15 minutes $)$ to suppress the potential methanogenic activity in the later experiment ${ }^{144} .200 \mathrm{mMC}$ of methanol, $1 \mathrm{ml} / \mathrm{L}$ vitamin solution and $1 \mathrm{ml} / \mathrm{L}$ trace element solution were added to the ASW after the heat shock to obtain the growth medium for the batch experiment. Yeast extract was omitted in this batch experiment. 


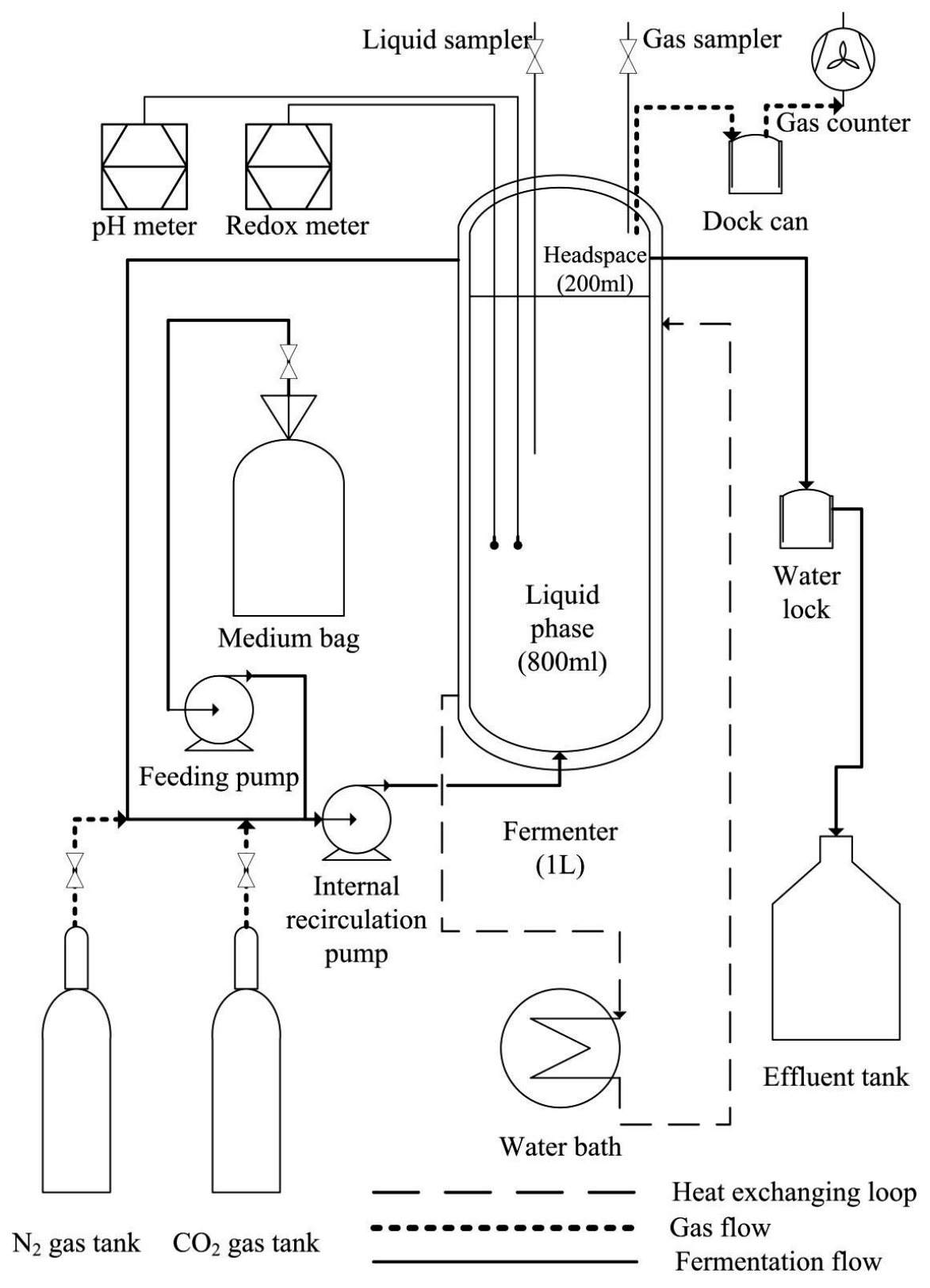

Figure S4.1. Reactor setup of the anaerobic bioreactor used in this study for performing continuous methanol chain elongation. 


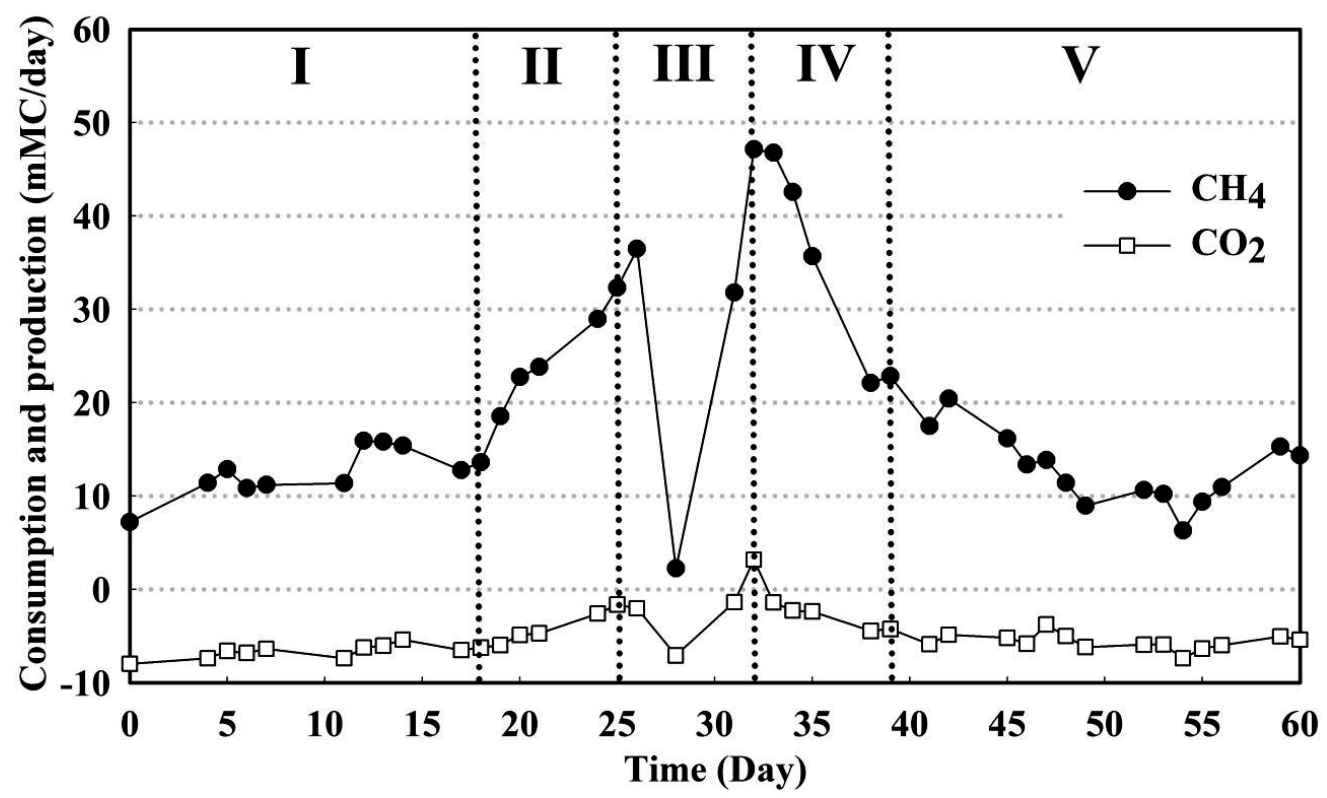

Figure S4.2. $\mathrm{CH}_{4}$ production and $\mathrm{CO}_{2}$ consumption rates over the entire operational period of the continuous methanol chain elongation. 


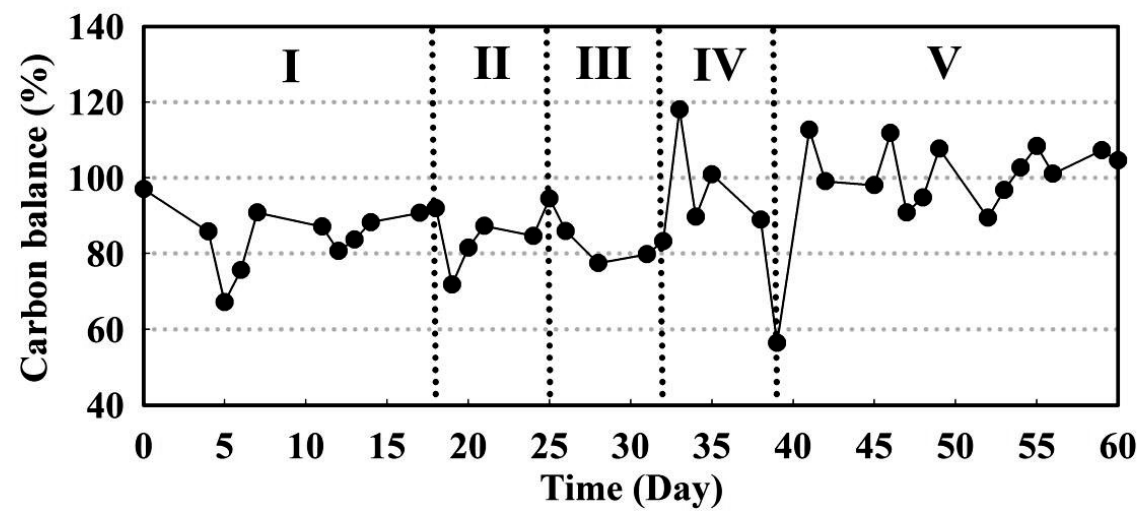

Figure S4.3. Overview of the carbon balance of the continuous experiment .

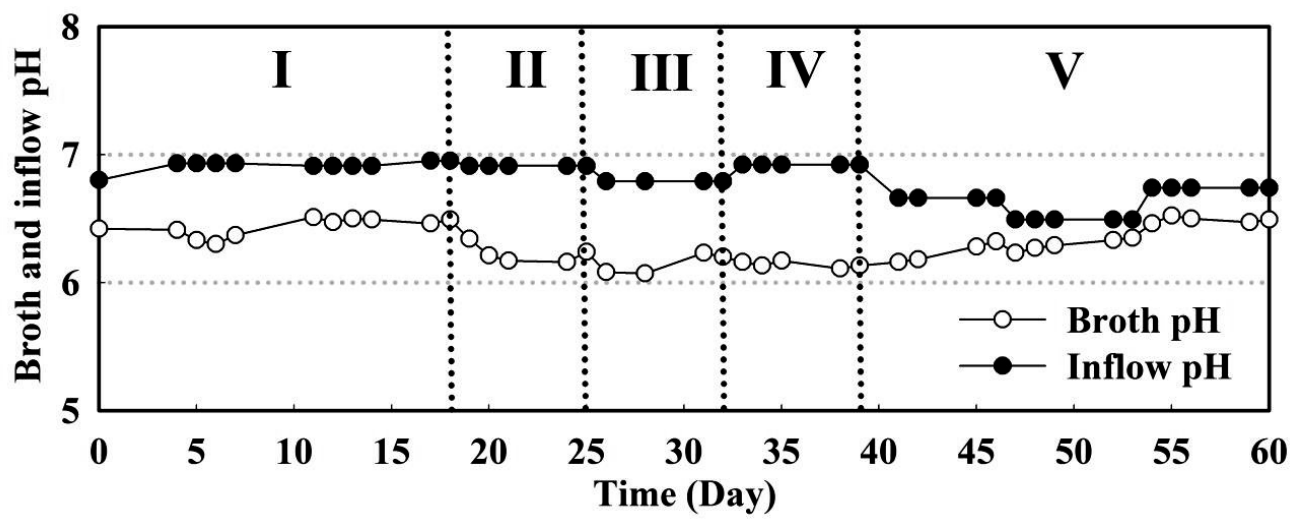

Figure S4.4. Overview of the broth $\mathrm{pH}$ and the inflow $\mathrm{pH}$ of the continuous experiment . 
Isobutyrate biosynthesis via methanol chain elongation 


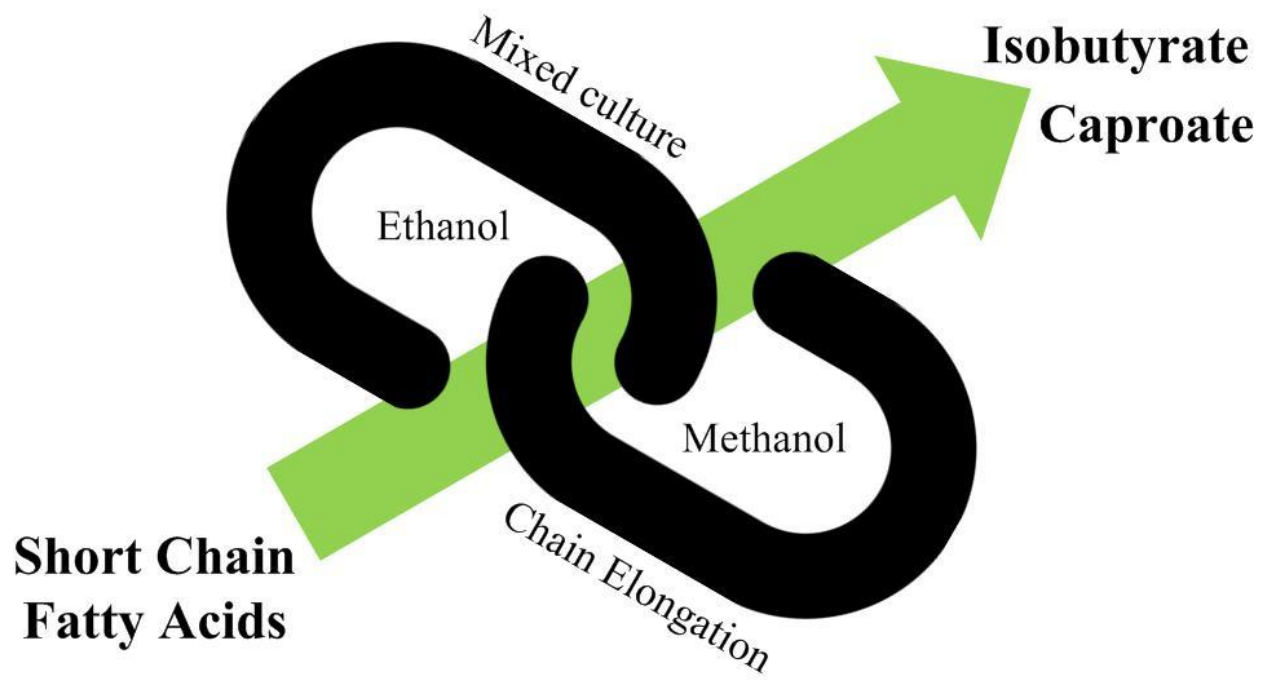




\title{
Chapter 5. Concurrent use of methanol and ethanol for chain-elongating short chain fatty acids into caproate and isobutyrate
}

\begin{abstract}
This study investigated the feasibility of using two electron donors, i.e. methanol and ethanol, for upgrading short chain fatty acids (SCFAs) into isobutyrate and caproate concurrently in chain elongation. Chain elongation is a novel bioprocess which utilises an open mixed culture fermentation to valorise organic waste. Caproate is an economically valuable chemical, which can be produced via chain elongation of ethanol and acetate. Isobutyrate is an existing platform chemical with a large market potential, and it was recently demonstrated to be a novel product from chain elongation of methanol and acetate. Supplying both methanol and ethanol in chain elongation was shown to simultaneously convert acetate and/or butyrate into caproate and isobutyrate, due to the use of an open mixed culture microbiome. Moreover, the butyrate supplement stimulated the caproate production rate to $2.6 \mathrm{~g} / \mathrm{L}$.day and induced isobutyrate production ( $1.5 \mathrm{~g} / \mathrm{L}$.day). Further increasing the ethanol feeding rate enhanced the direct use of butyrate for caproate production, which improved the caproate production rate to $6 \mathrm{~g} / \mathrm{L}$.day. Overall, the integration of two microbial pathways, i.e. ethanol and methanol chain elongation, in one reactor system to upgrad SCFAs was demonstrated. As such, chain elongation can be applied to valorise organic waste(water) streams into a wider variety of value-added biochemicals.
\end{abstract}

This chapter is to be submitted as: Chen, W.S.; Huang, S.; Strik, D. P. B. T. B.; Buisman, C. J. N. Concurrent use of methanol and ethanol for chain-elongating short chain fatty acids into caproate and isobutyrate. 


\subsection{Introduction}

Microbial chain elongation is an emerging biotechnology that converts the carbon materials found in waste streams into value-added biochemicals ${ }^{33}$, 34 . Chain elongation refers to the elongation of the carbon chain of a molecule. Examples of microbial chain elongation include homoacetogenesis, which converts $\mathrm{CO}_{2}$ to acetate, the succinate formation from glycerol and $\mathrm{CO}_{2}$ and the conversion of short chain fatty acids (SCFAs; saturated carboxylic acid with 2 to 5 carbons) into medium chain fatty acids (MCFAs; saturated carboxylic acid with 6 to 12 carbons $)^{34,41}$. The last example, i.e. chain elongation of SCFAs, is particularly interesting due to its ability to use an open mixed culture biocatalyst, its applicability to valorise complex organic waste streams, the high economic values and the potential separation of its endproducts, i.e. the MCFAs. This paper focuses on the microbial chain elongation of SCFAs using an open mixed culture reactor microbiome, which we refer to as chain elongation throughout the following text. The use of such a microbiome as a stable catalyst in chain elongation was demonstrated in several lab-scale systems, with various reactor configurations and is currently under development at a pilot-scale level ${ }^{33}$. The use of organic waste as the feedstock for chain elongation was also demonstrated in several studies ${ }^{38,39,145}$. The endproduct from chain elongation, i.e. the MCFAs, are value-added chemicals that can be used as feed additives ${ }^{67,68}$ or can serve as building blocks for several commodities ${ }^{26,65,106}$. Several

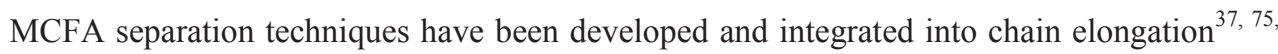
77. A pilot-scale microbial chain elongation system (ChainCraft B.V., The Netherlands) has also been recently established, which aims to convert supermarket food waste and ethanol into commercially-viable caproate ${ }^{33,145}$.

In recent years, research efforts are continuously dedicated to chain elongation, especially with regards to the expansion and investigation of its substrate and product spectrums ${ }^{33,70}$. 
The use of different substrates for both the electron donor and acceptor in chain elongation can lead to distinct product spectrums ${ }^{33,70}$, with a variety of studies completed on the topic. For example, Steinbusch et al. (2011) and Agler et al. (2012) investigated the use of evenchain SCFAs, i.e. acetate and butyrate, as the electron acceptors and ethanol as the electron donor, which yielded caproate as the main product ${ }^{32,37}$. Van Eerten-Jansen et al. (2013) used electricity as the electron donor to chain-elongate acetate into butyrate, caproate and caprylate $^{42}$. Grootscholten et al. (2013) used propionate as the electron acceptor and ethanol as the electron donor to synthesise valerate and heptanoate ${ }^{71}$. Zhu et al. (2015) and Kucek et al. (2016) demonstrated the use of lactate as both the electron donor and acceptor to produce caproate $^{43,44}$. Chen et al. (2016) employed methanol as the electron donor to chain-elongate acetate to butyrate, isobutyrate and caproate ${ }^{90,91}$. Isomerised fatty acids, e.g. isobutyrate, are an established platform chemical that has a much larger market than caproate ${ }^{57}$. Recently, Coma et al. (2016) also did a thorough investigation of several combinations of electron donor and acceptor in chain elongation in order to explore product diversity and to understand the microbial community linked to the versatile substrate combinations ${ }^{70}$.

Most of the aforementioned chain elongation studies supplied only one electron donor at a time for chain-elongating SCFAs, with the exception of two batch experiments ${ }^{32,91}$. Using two or more electron donors concurrently can be beneficial for the overall product yield, the extensive utilisation of the SCFA and the formation of novel products. In Steinbusch et al. (2011), hydrogen and ethanol were used simultaneously as electron donors for elongating acetate. The concurrent use of hydrogen and ethanol as electron donors led to a higher yield, a better product recovery efficiency and the formation of novel products, i.e. butanol, hexanol and caprylate ${ }^{32}$. In the same batch experiment, when ethanol was used as the sole electron donor, butanol and hexanol were not produced and less caproate was formed. Thus, the formation of hexanol and butanol could result from the biohydrogenation of caproate which 


\section{Chapter 5}

was produced via chain elongation of ethanol and acetate ${ }^{32,88}$. In Chen et al. (2016), methanol and ethanol were simultaneously available as the electron donors for chainelongating SCFAs in the acidified supermarket food waste. The ethanol was endogenous and formed due to the degradation of larger organic molecules, like glucose, during the hydrolysis and acidification of the supermarket food waste, while the methanol was added. The simultaneous presence of methanol and ethanol led to the formation of multiple valuable endproducts, i.e. isobutyrate, valerate, caproate and trace amount of isovalerate. Moreover, the formation of isovalerate was not reported in previous chain elongation studies, and it possibly formed via the isomerisation of valerate that was produced by chain elongation of ethanol and propionate $^{91}$.

Previously, Kenealy and Waselefsky (1985) examined the simultaneous use of methanol and ethanol during the growth of Clostridium kluyveri (a well-known ethanol chainelongating microorganism) ${ }^{146}$. Their results showed that, when methanol and ethanol were supplied simultaneously, C.kluyveri does not uptake methanol as an electron donor. The product formation and growth of C.kluveri slightly decreased compared to the use of ethanol as the sole electron donor ${ }^{146}$. On the other hand, Eubacterium limosum, the only known microorganism that chain-elongates methanol and SCFAs into butyrate, valerate and caproate, does not use ethanol as a substrate ${ }^{55}$. The consumption of methanol and ethanol observed in Chen et al. (2016) was suspected to occur via different microbial pathways, i.e. different microorganisms, within the mixed-culture reactor microbiome. This implies that two distinct microbial metabolisms can be integrated to simultaneously upgrade SCFAs in organic waste into multiple valuable biochemicals via the use of a mixed culture microbiome. This could be an advantage of using an open mixed culture microbiome ${ }^{20}$. 
In summary, the simultaneous use of two or more electron donors for chain-elongating SCFAs could be beneficial due to the extensive utilisation of SCFAs, the formation of multiple valuable products and the potential formation of novel products. Nevertheless, the concurrent use of two electron donors for chain-elongating SCFAs was never demonstrated in a continuous operation. Additionally, the production of valuable products besides the major product was shown to be limited. In Steinbusch et al. (2011), caproate was the major product, amounting to 100 millimole carbon $(\mathrm{mmoleC}) / \mathrm{batch}$, while caprylate amounted to 20 $\mathrm{mmoleC} / \mathrm{batch}$ and butanol and hexanol to 5 and $1 \mathrm{mmoleC} / \mathrm{batch}^{32}$. In Chen et al. (2016), isobutyrate was the major product, amounting to $30 \mathrm{mmoleC} / \mathrm{batch}$, while valerate amounted only to 10 and caproate to $5 \mathrm{mmoleC} / \mathrm{batch}$ only. It remains uncertain whether the ethanol chain elongation did occur and contribute to the organic acid formation, as the formation of valerate and caproate could have occurred via methanol chain elongation ${ }^{47,90,91}$.

In this study, we examined the feasibility of using methanol and ethanol in a single reactor system to chain-elongate SCFAs simultaneously and continuously. We anticipated that isobutyrate and caproate will be produced simultaneously, and the caproate production will be in a higher concentration and rate than the previous study ${ }^{91}$ due to the increased ethanol concentration and feeding rate. Moreover, the effect of butyrate supplement as an additional SCFA towards chain elongation was examined in this study. Butyrate is a common intermediate in hydrolysed/acidified organic wastes besides acetate ${ }^{85}$, and the presence of butyrate in higher concentrations appears to be essential for isobutyrate formation ${ }^{91}$. The outcome of this study may serve as an example of stimulating two microbial pathways for extensively upgrading SCFAs into multiple value-added platform chemicals, within a single reactor microbiome in a continuous operation. 


\subsection{Methodology}

\subsubsection{Continuous bioreactor setup and operation}

A 1-L anaerobic bioreactor was operated in the continuous mode for 118 days during this study (Figure 5.1). The setup of this anaerobic bioreactor was identical to the one used in a previous methanol chain elongation study, except for the installation of an automated $\mathrm{pH}$ controller, which was added for this study ${ }^{91}$. This addition (LIQUISYS M, Endress+Hauser B.V., NL; modified by Elektronica ATV, WUR, NL) was installed on day 38 in order to maintain a stable $\mathrm{pH}$, as the organic acids produced via chain elongation continue to acidify the fermentation broth. Potassium hydroxide $(\mathrm{KOH} ; 1 \mathrm{M})$ was dosed by the $\mathrm{pH}$ controller to maintain the broth $\mathrm{pH}$ around $6.6 \pm 0.1$. The entire continuous operational period consisted of the start-up phase and five experimental phases with different variables tested, as shown in Table 5.1. The hydraulic retention time (HRT) was set as 37 hours; the actual HRT was calculated by measuring the weight of the medium bag before and after the medium replacement. The temperature was kept at $35^{\circ} \mathrm{C}$ using a water bath (Fisher Scientific Polystat 37, USA); the actual temperature was regularly measured and examined using a thermometer (Fisher Scientific Traceable digital thermometer, USA). 
Table 5.1. Conditions for the start-up phase and the five experimental phases of the continuous bioreactor operation.

\begin{tabular}{|c|c|c|c|c|c|c|c|c|}
\hline \multicolumn{9}{|c|}{ Variables used (Changes are in bold font) } \\
\hline \multirow{2}{*}{$\begin{array}{l}\text { Phases } \\
\text { Period (day) }\end{array}$} & \multicolumn{2}{|c|}{ Strat-up } & \multicolumn{2}{|r|}{ I } & \multirow{2}{*}{$\begin{array}{l}\text { II } \\
63-83\end{array}$} & \multirow{2}{*}{$\begin{array}{l}\text { III } \\
84-97\end{array}$} & \multirow{2}{*}{$\begin{array}{l}\text { IV } \\
98-104\end{array}$} & \multirow{2}{*}{$\begin{array}{l}\mathrm{V} \\
105-118\end{array}$} \\
\hline & $0-6^{\dagger}$ & $7-23^{*}$ & $24-40$ & $40-62$ & & & & \\
\hline $\begin{array}{l}\text { Ethanol feeding rate } \\
\text { (mmoleC/L.day) }\end{array}$ & - & 140 & 140 & 140 & 140 & 280 & - & 140 \\
\hline $\begin{array}{l}\text { Butyrate feeding rate } \\
\text { (mmoleC/L.day) }\end{array}$ & - & - & - & - & 480 & 480 & 480 & - \\
\hline $\begin{array}{l}\mathrm{CO}_{2} \text { feeding rate } \\
\text { (mmole/day) }\end{array}$ & 10 & 10 & 20 & 20 & 20 & 20 & 20 & 20 \\
\hline Automated $\mathrm{pH}$ control $^{¥}$ & - & - & - & $\mathbf{v}$ & $\mathrm{V}$ & $\mathrm{V}$ & $\mathrm{v}$ & $\mathrm{V}$ \\
\hline $\begin{array}{l}{ }^{\dagger} \text { Vitamin } \mathrm{B} 12 \text { was inc } \\
\text { rate }=240 \mu \mathrm{g} / \mathrm{L} \text {.day) fr } \\
\mathrm{ml} / \mathrm{minute} \text { to } 300 \mathrm{ml} / \mathrm{mi} \\
\text { on day } 38 \text {, and a stable p }\end{array}$ & $\begin{array}{l}\text { ase } \\
\text { d } \\
\text { te }\end{array}$ & $\begin{array}{l}\text { m } 13 \\
\text { onws } \\
\text { day } \\
\text { intair }\end{array}$ & $\begin{array}{l}\text { L ( } 1 \text { su } \\
\text { "Inte } \\
\text { onwarc } \\
\text { rom d }\end{array}$ & $\begin{array}{l}\text { ly ra } \\
\text { al re } \\
{ }^{\ddagger} \mathrm{Th} \\
40 \mathrm{c}\end{array}$ & $\begin{array}{l}7.8 \\
\text { latio } \\
\text { toma } \\
\text { ards. }\end{array}$ & $\begin{array}{l}\text { g/L.da } \\
\text { rate } \mathrm{v} \\
\mathrm{d} \mathrm{pH}\end{array}$ & $\begin{array}{l}\text { to } 400 \mu \\
\text { increase } \\
\text { troller } w\end{array}$ & $\begin{array}{l}\text { L (supply } \\
\text { from } 150 \\
\text { installed }\end{array}$ \\
\hline
\end{tabular}

\subsubsection{Inoculum and medium}

The inoculum used in this study was a reactor microbiome derived from a previous chain elongation reactor fed with acetate and ethanol ${ }^{36}$. Before starting this experiment, the reactor microbiome was kept in a continuous reactor (the same one used in this study but without an automated $\mathrm{pH}$ controller) for 200 days. During this 200-day incubation, the reactor microbiome was fed with a medium containing acetate, ethanol and methanol. The main products were caproate and butyrate, and the main substrate consumed were ethanol and acetate. The methanol supplied was hardly consumed during the entire 200-day incubation (data not shown). After the 200-day incubation, the feeding was stopped and the reactor was 
switched to batch-mode for 50 days. In this study, the continuous reactor was restarted by feeding a medium containing methanol and acetate as the main carbon source.

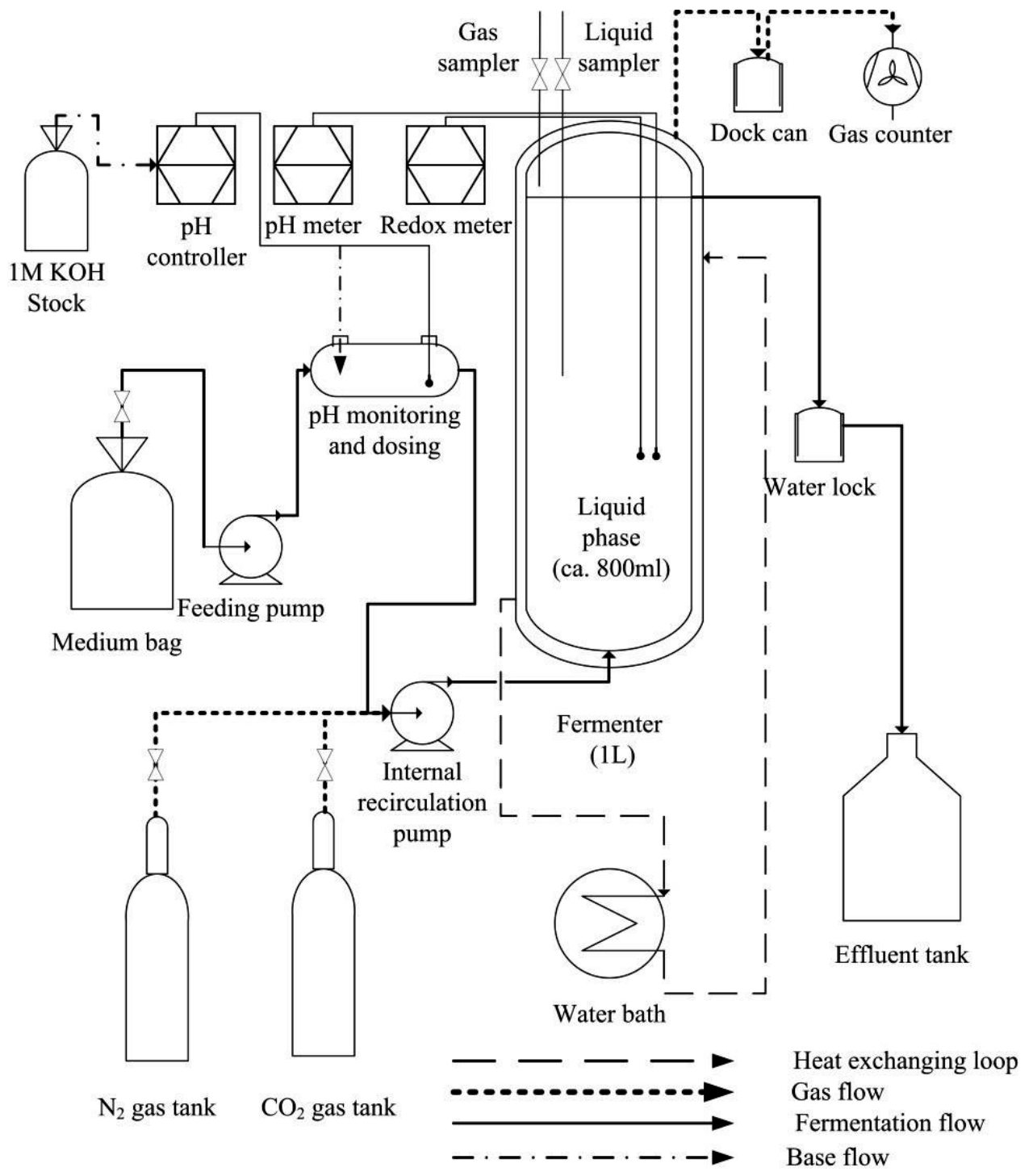

Figure 5.1. The schematic illustration of the continuous bioreactor used in the present study.

A synthetic medium was used to perform the continuous chain elongation, which contained the basal medium and the addition of ethanol, butyrate or a mixture of both (see Table 5.1). The basal medium contained methanol - $6.4 \mathrm{~g} / \mathrm{L}$, acetate - $6.0 \mathrm{~g} / \mathrm{LNH}_{4} \mathrm{H}_{2} \mathrm{PO}_{4}-3.6 \mathrm{~g} / \mathrm{L}$, 
$\mathrm{MgCl}_{2} .6 \mathrm{H}_{2} \mathrm{O}-0.33 \mathrm{~g} / \mathrm{L}, \mathrm{MgSO}_{4} .7 \mathrm{H}_{2} \mathrm{O}-0.2 \mathrm{~g} / \mathrm{L}, \mathrm{CaCl}_{2} .2 \mathrm{H}_{2} \mathrm{O}-0.2 \mathrm{~g} / \mathrm{L}, \mathrm{KCl}-0.15 \mathrm{~g} / \mathrm{L}$, yeast extract - $1 \mathrm{~g} / \mathrm{L}$, Vitamin solution $1 \mathrm{ml} / \mathrm{L}$ and trace element solution $1 \mathrm{ml} / \mathrm{L}^{113}$. In different experimental phases, various concentrations of ethanol, butyrate or both were added to the synthetic medium to test the effect of alternative feeding rate, as shown in Table 5.1. During the bioreactor operation, the synthetic medium was stored anaerobically in a refrigerator and replaced every week. Gaseous $\mathrm{CO}_{2}$ was continuously supplied to the reactor as another substrate for sustaining biomass growth. The $\mathrm{CO}_{2}$ supply rate was controlled by a mass flow controller (Brooks mass flow controller 5850E, USA) at 240 or $480 \mathrm{ml} \mathrm{CO}$ /day (see Table $5.1)$.

\subsubsection{Sampling and Analyses}

Liquid samples $(1 \mathrm{ml})$ were taken from the fermentation broth 5 times a week and from the synthetic medium every time it was prepared (approximately once per week). Gas samples were taken with a syringe from the headspace every two to three days. All liquid samples were analysed with two gas chromatographs (GCs) using the standardised methods that were employed in several previous chain elongation studies ${ }^{32,90}$. One GC (HP5890, USA) analysed the concentration of the saturated carboxylic acids including acetate, propionate, isobutyrate (2-Methylpropanoate), butyrate, isovalerate (3-Methylbutanoate), valerate, isocaproate (4Methylpentanoate), caproate, heptanoate and caprylate. The other GC (HP5890, USA) analysed the concentration of methanol and ethanol. The gas samples were analysed immediately after their extraction from the headspace of the bioreactor. One GC (Shimadzu GC-2010, Japan) was used to measure the $\mathrm{O}_{2}, \mathrm{~N}_{2}, \mathrm{CH}_{4}$ and $\mathrm{CO}_{2}$ concentrations, whilst the other GC (HP5890, USA) was used to measure the $\mathrm{H}_{2}$ concentration in the gas samples. 


\subsection{Results and discussion}

\subsubsection{Concurrent use of methanol and ethanol for upgrading SCFAs}

The concurrent consumption of methanol and ethanol was observed in Phase I with the butyrate and caproate found to be the main products (Figure 5.2a). Both methanol and ethanol were used for chain-elongating acetate into butyrate and caproate, as the total amount of carbon consumed (in terms of ethanol and acetate), was not sufficient to provide the total amount of carbon in the produced butyrate and caproate. Nevertheless, most of the produced organic acids were likely formed via chain elongation of ethanol and acetate, while methanol contributed less.

In the previous study on chain elongation of methanol and acetate, caproate was produced at both a low rate $(9 \mathrm{mmoleC} / \mathrm{L}$.day) and low concentration $(15 \mathrm{mMC}$ or $0.3 \mathrm{~g} / \mathrm{L} ; \mathrm{mMC}=$ millimolar carbon) ${ }^{90}$. Most likely, the observed caproate in Phase I of this study (up to 81 mmoleC/L.day) was formed mainly via chain elongation of ethanol and acetate. As for the butyrate, in the previous study conducted under a similar condition but without ethanol feeding, the maximal butyrate production rate was $60 \mathrm{mmoleC} / \mathrm{L}$.day, whilst during Phase I it rose to $145 \mathrm{mmoleC} / \mathrm{L} \cdot \mathrm{day}^{90}$. This supports the assumption that more than half of the butyrate produced in Phase I was from chain elongation of ethanol and acetate. 
(a)
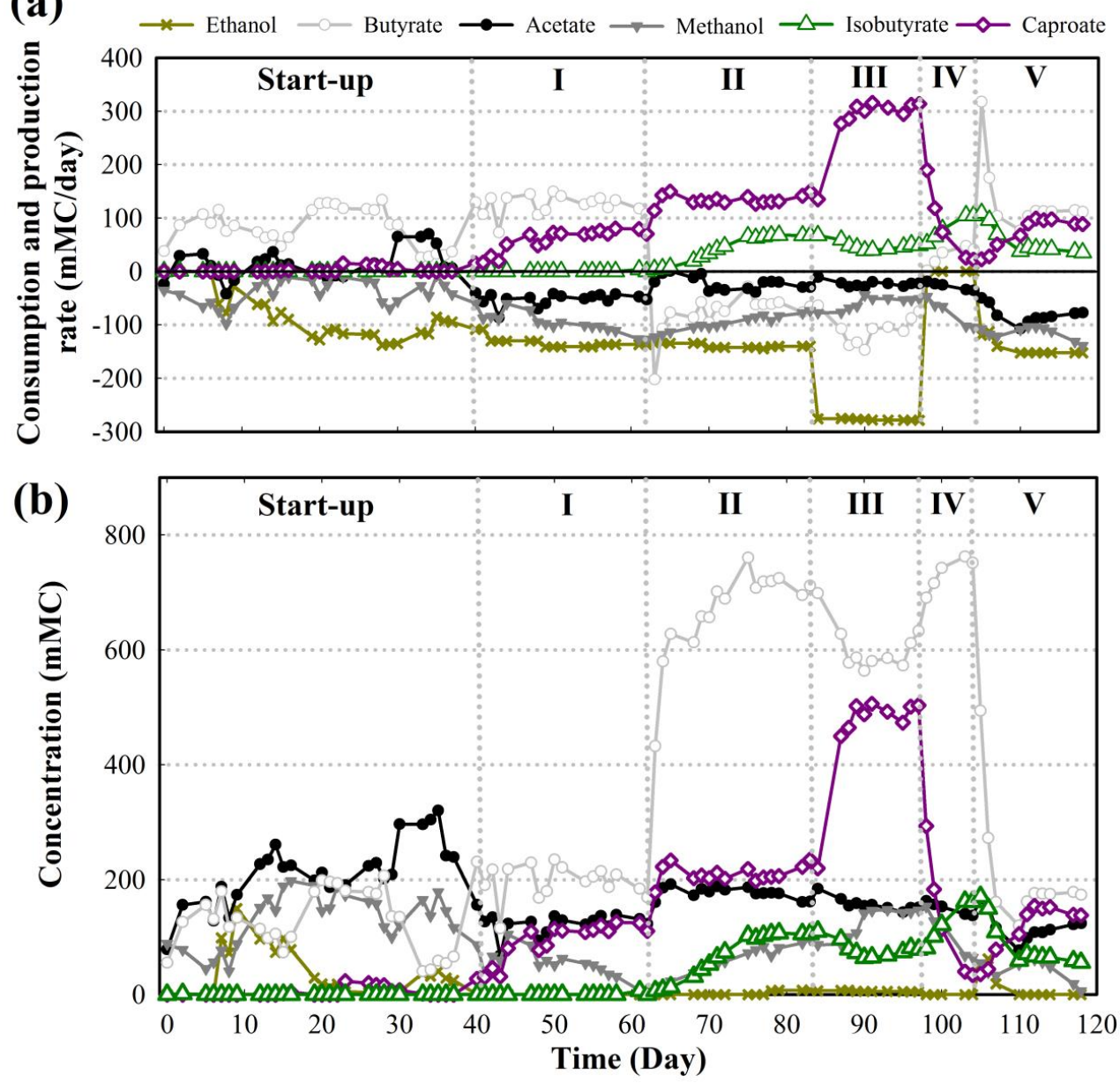

Figure 5.2. The production and consumption profile (a) and the concentration profile (b) of all products and substrates in the fermentation broth during the entire operational period of the chain elongation reactor, using methanol and/or ethanol as the electron donors (Temperature $=35^{\circ} \mathrm{C}, \mathrm{HRT}=37$ hours).

Figure $5.2 \mathrm{~b}$ showed the concentration profile of the entire fermentation experiment. In Phase I, the ethanol concentration was always under the detection limit, showing that ethanol was completely used. From day 40, the caproate concentration gradually increased until day 50 , and from this point it remained relatively stable until day $62(110 \mathrm{mMC})$. This could be 


\section{Chapter 5}

an indication for the enrichment of ethanol chain-elongating microorganisms. In contrast, from day 40 to 50 , there was residual methanol found in the fermentation broth. The methanol concentration gradually decreased from day 44 due to the increasing methanol consumption rate. The consumed methanol was probably converted into methane, as the methane production rate gradually increased from day 44, following a similar trend as that of the methanol consumption rate (Figure 5.3). Moreover, comparing day 44 with day 62 , the methanol consumption rate had increased by approximately $40 \mathrm{mmoleC} / \mathrm{L}$.day (Figure 5.2a) and there was no residual methanol concentration (Figure 5.2b). The methane production rate was also increased by $30 \mathrm{mmole} /$ day from day 44 to day 62 (Figure 5.3). It is likely that all the increased methanol consumption was converted into methane as there are methylotrophic methanogens present, which are known to metabolise methanol and even prefer methanol over other substrates available in this study, e.g. acetate ${ }^{147}$. Overall, in Phase I, methanol and ethanol were both used for chain-elongating acetate into longer fatty acids. However, methanol contributed less than ethanol to the organic acid formation and contributed considerably to the methane production. From Phase II, several strategies for improving the methanol use for organic acid production were investigated, as discussed in section 5.3.2. 
(a)
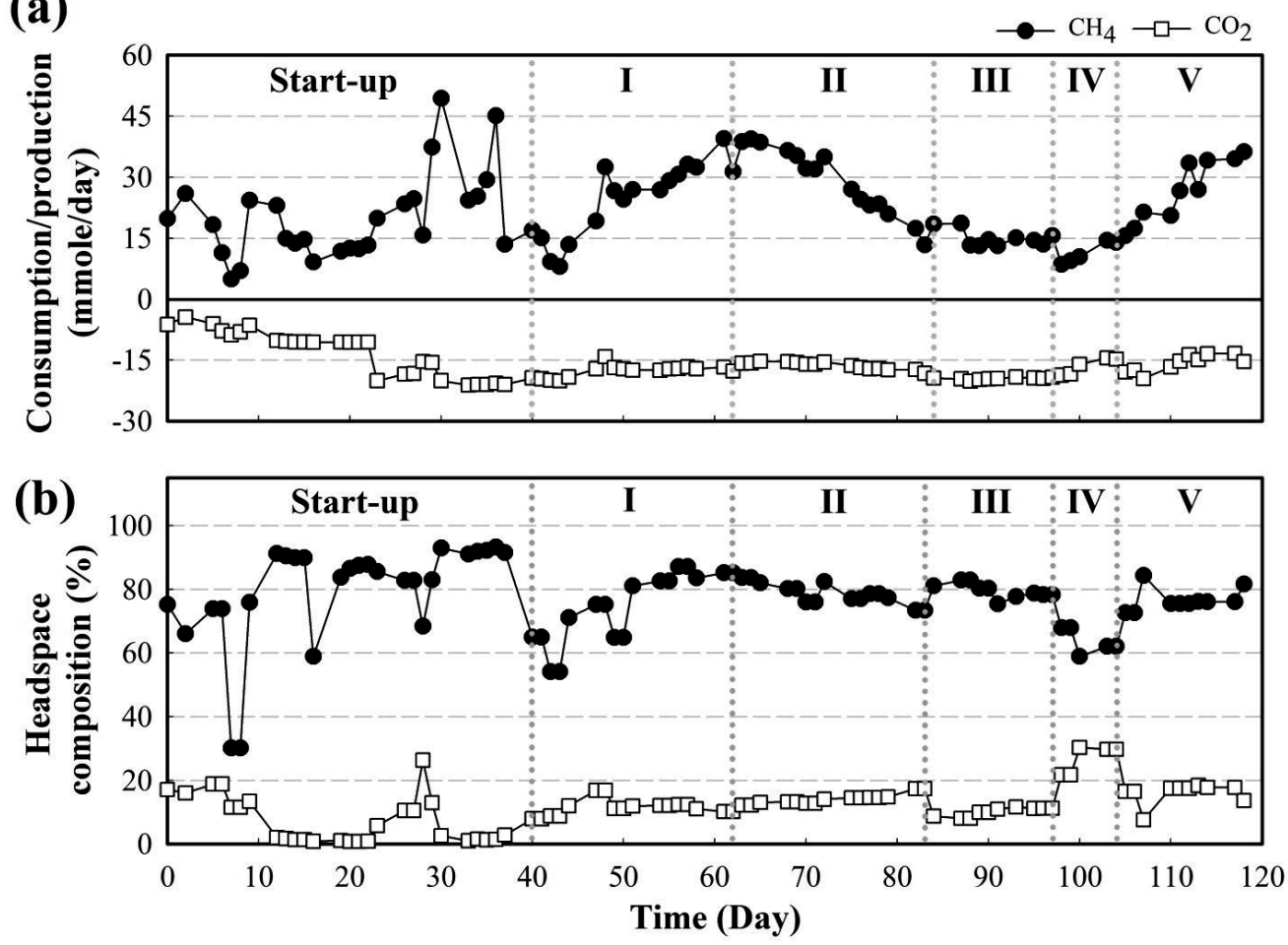

Figure 5.3. The profile of the total $\mathrm{CH}_{4}$ and $\mathrm{CO}_{2}$ production/consumption (a) and the headspace $\mathrm{CH}_{4}$ and $\mathrm{CO}_{2}$ compositions (b) throughout the entire operational period.

Comparing the start-up phase and Phase I, pH control at $6.6 \pm 0.1$ was shown to be essential for the concurrent use of methanol and ethanol for chain-elongating SCFAs. In the start-up phase, the ethanol supplement in methanol chain elongation constantly caused $\mathrm{pH}$ drops (Figure 5.4). In the first six days of the start-up phase, ethanol was not supplied, and the $\mathrm{pH}$ was around 6.2 without any external $\mathrm{pH}$ control means. The consumed methanol was mainly converted into acetate and butyrate via methanol chain elongation or into methane via methylotrophic methanogenesis ${ }^{90,} 91$. From day 7 when ethanol was added, ethanol consumption gradually started and methanol consumption gradually stopped. Chain elongation of acetate and ethanol into butyrate became the dominant reaction, and $\mathrm{pH}$ dropped to below 5.5 constantly (Figure 5.3). The $\mathrm{pH}$ was adjusted to 6.3 by adding $\mathrm{KOH}$ to 


\section{Chapter 5}

the bioreactor on days 15,21 and 28 ; nevertheless, the $\mathrm{pH}$ dropped quickly after each $\mathrm{pH}$ adjustment. The $\mathrm{pH}$ reduction could be due to proton formation during the ethanol chain elongation $^{34}$ and could inhibit the methanol chain-elongating microorganisms. As a result, a stable methanol consumption was no longer observed during the the start-up phase, following the $\mathrm{pH}$ drop. When the $\mathrm{pH}$ dropped below 5.5, e.g. on day 34 , chain elongation of ethanol to butyrate was also no longer observed. Both methanol and ethanol chain elongation started again when an automated $\mathrm{pH}$ controller was installed to constantly maintain the $\mathrm{pH}$ at $6.6 \pm$ 0.1. The same $\mathrm{pH}$ range was maintained for the rest of the experimental period to ensure the activities of both methanol and ethanol chain elongation.

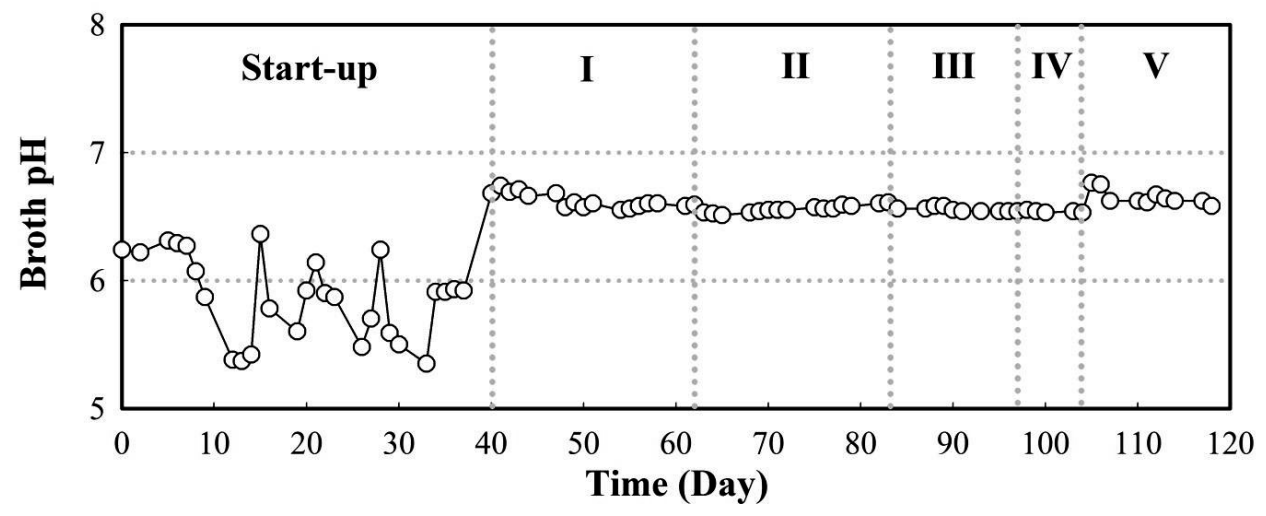

Figure 5.4. The profile of broth $\mathrm{pH}$ during the entire experimental period.

\subsubsection{Concurrent caproate and isobutyrate production and its relation to ethanol and butyrate feeding rates}

5.3.2.1 Feeding butyrate and ethanol induces concurrent production of caproate and isobutyrate

In Phase II, butyrate was fed to the reactor directly, with the same feeding rate used in the previous methanol chain elongation study ${ }^{91}$. After feeding butyrate, the concurrent production of caproate and isobutyrate was observed (Figure 5.2). The isobutyrate production provided further support for the concurrence of two distinct microbial pathways. Isomerised organic 
acids were not reported as a product from ethanol chain elongation, and this was only observed in methanol chain elongation. Moreover, the caproate production rates observed in Phase II of this study were also much higher than those reported in previous methanol chain elongation studies. Chen et al. (2016) observed simultaneous isobutyrate $(81 \pm 6$ mmoleC/L.day), butyrate $(10 \pm 28 \mathrm{mmoleC} / \mathrm{L}$.day $)$ and caproate $(9 \pm 1 \mathrm{mmoleC} / \mathrm{L}$.day $)$ production in a continuous methanol chain elongation fed with a synthetic medium containing methanol, acetate and butyrate but no ethanol ${ }^{91}$. The reported production rate was an average derived from a 10-day period (approximately 6 HRT) when a steady production of isobutyrate and caproate was maintained. To compare, we took a 7-day period in each phase of the study (with the exception of Phase IV, which was too short), during which a steady organic acid production was maintained. During this period we calculated the average production and consumption rates (see Figure 5.5). The isobutyrate production rate $(66 \pm 2$ mmoleC/L.day from day 76 to 82 ) in Phase II of this study was slightly lower than that in the previous study ${ }^{91}$. The caproate production rate in Phase II (132 $\pm 6 \mathrm{mmoleC} / \mathrm{L}$.day), on the other hand, increased by 13 times when compared to the previous study ${ }^{91}$.

The operational conditions and medium composition in Chen et al. (2016) were similar to that in this study, with the exception of the ethanol supplement and an increased $\mathrm{CO}_{2}$ feeding rate in this study. The ethanol feeding was mainly responsible for the elevated caproate production rate in Phase II, as the increased $\mathrm{CO}_{2}$ feeding rate (from 10 mmole/day in the previous study to $20 \mathrm{mmole} /$ day in this study) was far less than the increased caproate production rate. The ethanol feeding stimulated the chain elongation of ethanol and acetate into caproate while the chain elongation of methanol and acetate into isobutyrate occurred continuously (Phases II, III and V in Figure 5.2a). As mentioned in section 5.1, the simultaneous consumption of methanol and ethanol by a single microorganism has not been reported. In contrast, the use of a mixed culture allows the coexistence of two distinct 


\section{Chapter 5}

microbial pathways as observed in Phases II, III and V of this study. A previous study by Chen et al. (2016) also suggested the coexistence of methanol and ethanol chain-elongating microorganisms in a reactor microbiome taken from an ethanol chain elongation bioreactor where methanol was not supplied ${ }^{90}$. The methanol chain-elongating microorganisms were suspected to grow on $\mathrm{H}_{2}$ and $\mathrm{CO}_{2}$ in the ethanol chain elongation reactor, and to gradually adopt to grow on methanol and acetate when methanol was present in the environment ${ }^{90}$. Therefore, the observed concurrent conversion of methanol and ethanol with SCFAs into higher organic acids possibly occurred via two distinct microbial pathways. This further supports our aim of the study, i.e. the stimulation of two distinct metabolisms in one reactor microbiome for extensively upgrading VFAs to isobutyrate and caproate concurrently.

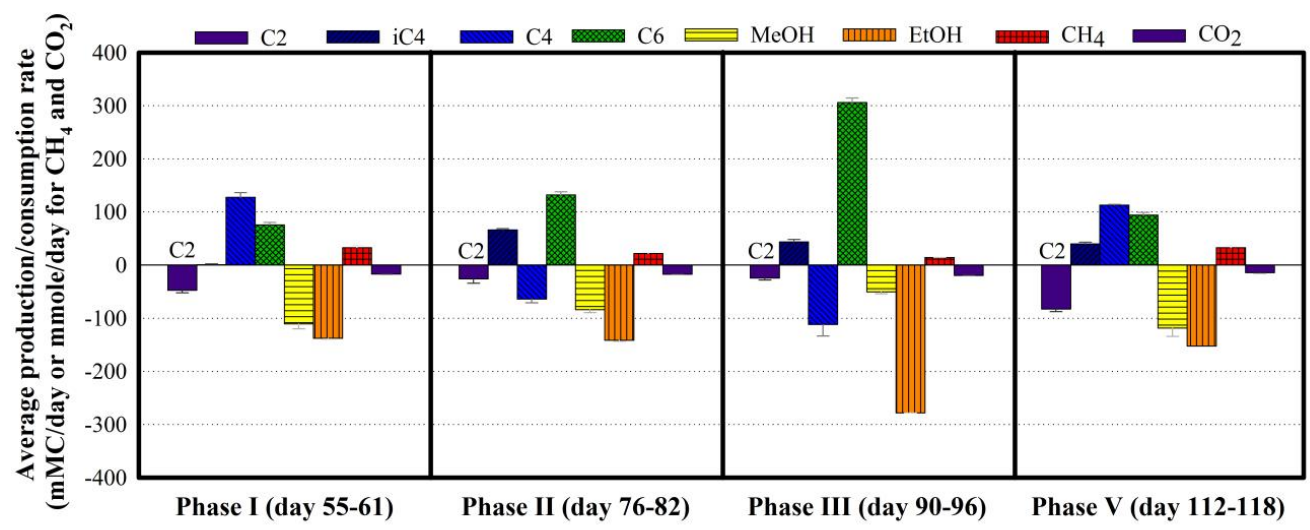

Figure 5.5. The average production and consumption rate of all measured compounds, including that in the broth and in the headspace, during the relatively steady period in each phase. The relatively steady period is indicated in the figure. The vertical bar in each phase stands for (from left to right): $\mathrm{C} 2$, acetate; iC4, isobutyrate; $\mathrm{C} 4$, butyrate; $\mathrm{C} 6$, caproate; $\mathrm{MeOH}$, methanol; EtOH, ethanol; $\mathrm{CH}_{4}$, methane; $\mathrm{CO}_{2}$, carbon dioxide. 


\subsubsection{Increasing ethanol feeding rate stimulates chain elongation of butyrate and ethanol to caproate}

In Phase III, the ethanol feeding rate was increased from 140 to $280 \mathrm{mmoleC} / \mathrm{L}$.day. This increased the ethanol consumption rate by $136 \mathrm{mmoleC} / \mathrm{L}$.day, the caproate production rate by $174 \mathrm{mmoleC} / \mathrm{L}$.day and the butyrate consumption rate by $48 \mathrm{mmoleC} / \mathrm{L}$.day (Figure 5.5). The incrase in caproate production rate was $38 \mathrm{mmoleC} / \mathrm{L}$.day more than the increased ethanol consumption rate. This indicates that the increased caproate production rate partly resulted from the increased butyrate consumption rate, and implies the direct use of butyrate and ethanol for producing caproate by the microorganisms. In other words, the increased ethanol feeding rate enhanced the chain elongation of butyrate to caproate using ethanol as an electron donor, under the condition that a high butyrate concentration was present in the bioreactor. To our best knowledge, direct chain elongation of butyrate and ethanol into caproate in a mixed culture fermentation was reported previously but only in a batch operation $^{70}$. This study demonstrated the continuous chain elongation of butyrate and ethanol into caproate in a mixed culture condition. The direct use of butyrate as a starting material for producing MCFAs is more beneficial than using acetate, because the ethanol required for chain-elongating acetate to butyrate is avoided.

The ethanol feeding rate was increased in Phase III to examine whether the production rates of isobutyrate and caproate could be further enhanced. Previously, Chen et al. (2016) tried to increase the methanol feeding rate to $300 \mathrm{mmoleC} / \mathrm{L}$.day, which seemed to inhibit the methanol chain-elongating microorganisms, as both methanol consumption and butyrate production rates decreased considerably ${ }^{91}$. As a result, the ethanol feeding rate was increased instead of the methanol feeding rate during this study. A higher ethanol feeding rate may increase the caproate and butyrate production rate via stimulating the chain elongation of ethanol and acetate. The consequentially increased endogenous butyrate concentration may then enhance the isobutyrate formation. The selected ethanol feeding rate and concentration 


\section{Chapter 5}

were also used in previous studies, where the ethanol chain-elongating activity was not inhibited $^{35,36}$.

The increased ethanol feeding rate did increase the caproate production rate, but not the butyrate production rate or concentration (Figure 5.5 and Figure 5.2b). Moreover, it decreased both methanol consumption and isobutyrate production rates by $34 \mathrm{mmoleC} / \mathrm{L}$.day and $23 \mathrm{mmoleC} / \mathrm{L}$.day, respectively (Figure 5.5), which may be an indication of the decreased methanol chain elongation activity. From a bioprocess point of view, there are several possible causes to the decreased activity of methanol chain elongation. These include competition for substrates e.g. acetate or butyrate and the competition and/or availability of (specific) nutrients, e.g. amino acids (provided in the form of yeast extract), trace elements and vitamins, as well as the inhibitory effect of ethanol and caproate. The competition for butyrate as a substrate is a plausible cause, as the isobutyrate production rate gradually decreased, along with the decreased butyrate concentration (days 84 -89; Figure 5.2). In the previous study and under a similar experimental condition, the isobutyrate production rate seemed to correlate positively with the in-situ broth butyrate concentration ${ }^{91}$. The availability of other substrates, e.g. methanol, $\mathrm{CO}_{2}$ and acetate, is less likely to be a limiting factor for isobutyrate production or methanol chain elongation, because they were sufficiently available in the environment (Figure 5.2b and 5.3b). The micronutrient may also be limiting the methanol chain elongation activity or the isobutyrate production rate. Previously, Grootscholten et al. (2014) found that the amount of yeast extract addition could be a limiting factor for a high-rate chain elongation process ${ }^{35}$. Although the caproate production rate was not as high as that achieved by Grootscholten et al. (2013), the methanol chain-elongating microorganisms in this study might also require yeast extract for growth, which could become a limiting factor when ethanol chain elongation activity was high ${ }^{35}$. In this study, the effect of increasing micronutrient supplement was not investigated; nevertheless, increasing 
the micronutrient loading rate may help improve the methanol chain elongation. The inhibitory effect of caproate or ethanol may be another cause. Inhibition of a high caproate concentration on methanol chain-elongating microorganisms was reported ${ }^{148}$. Pacaud et al. (1986) investigated the inhibition effect of various SCFAs and caproate on the growth of E.limosum. It was reported that for E.limosum, a threshold inhibition concentration of each tested organic acid exists, above which the inhibition effect occurs and increases linearly with the increasing organic acid concentration. The threshold inhibition concentration of the organic acid decreases, along with the increasing number of carbon atoms of the organic acid. Based on Pacaud et al. (1986), the threshold inhibition concentration of caproate for E.limosum is about $34 \mathrm{mMC}$, and a $50 \%$ growth rate inhibition is expected at a caproate concentration of around $540 \mathrm{mMC}^{148}$. Assuming that the methanol chain-elongating microorganism in this study was E.limosum or had a similar metabolism, the caproate concentration in Phase III, c.a. $500 \mathrm{mMC}$, approximated the $50 \%$ inhibitory concentration, which could decrease the growth rate of E.limosum to $0.058 \mathrm{~h}^{-1}$. Considering the 37-hour HRT in this study, the methanol chain-elongating microorganisms, though inhibited, could not be washed out. The inhibitory effect of caproate is unlikely a main cause to the decreased methanol chain elongation activity. The inhibitory effect of ethanol is also an unlikely possible cause, as there was hardly any residual ethanol concentration in the bioreactor (Figure 5.2b). Overall, despite the decreased activity of methanol chain elongation, it is still remarkable that increasing the ethanol feeding rate stimulated the use of butyrate as a starting material for ethanol chain elongation to produce caproate, and that chain elongation of methanol and acetate/butyrate to isobutyrate still occurred under such high ethanol feeding rate. 


\section{Chapter 5}

\subsubsection{Caproate production rate decreased substantially after stopping ethanol feeding}

In Phase IV, the ethanol feeding was stopped. The caproate production rate decreased rapidly, from $314 \mathrm{mmoleC} / \mathrm{L}$.day (503 $\mathrm{mMC}$ in concentration) on day 97 to 26 mmoleC/L.day (40 mMC) on day 103. The significant decrease in caproate production rate was anticipated, as the chain elongation of methanol and SCFAs to caproate never exceeded a concentration of $40 \mathrm{mMC}$ in either a pure or mixed culture incubation ${ }^{47,90,91}$. Lindley et al. (1987) demonstrated the caproate production from methanol (100mMC), butyrate (1600 $\mathrm{mMC})$ and $\mathrm{CO}_{2}(15 \%$ in the headspace $)$ up to $38 \mathrm{mMC}$ in a batch incubation of a pure culture E.limosum $^{47}$. Chen et al. (2016) demonstrated the continuous caproate production from methanol $(200 \mathrm{mMC})$, acetate $(200 \mathrm{mMC})$, butyrate $(800 \mathrm{mMC})$ and $\mathrm{CO}_{2}(20 \%$ in the headspace) up to $15 \mathrm{mMC}(10 \mathrm{mmoleC} / \mathrm{L}$.day in rate) with a mixed culture microbiome under a non-sterile condition ${ }^{91}$. In Phase IV of this study, where the operational condition was similar to that of Chen et al. (2016), the caproate production was still maintained at around 35 mmoleC/L.day (22 mmoleC/L.day in rate), taking day 104 as an example. The isobutyrate production rate (105 mmoleC/L.day), methanol and acetate consumption rates (105 and 35 mmoleC/L.day) are all higher in Phase IV of this study when compared with that of Chen et al. (2016; $81 \mathrm{mmoleC} / \mathrm{L}$.day for isobutyrate production rate, 60 and $26 \mathrm{mmoleC} / \mathrm{L}$.day for methanol and acetate consumption rates) ${ }^{91}$. The only different operational condition between Phase IV of this study and that in Chen et al. (2016) was the $\mathrm{CO}_{2}$ supplementation rate, i.e. $20 \mathrm{mmole} /$ day in this study versus $10 \mathrm{mmole} /$ day in Chen et al. (2016). The higher $\mathrm{CO}_{2}$ supplementation rate resulted in a higher $\mathrm{CO}_{2}$ consumption rate, i.e. $10 \mathrm{mmole} /$ day more $\mathrm{CO}_{2}$ was consumed in Phase IV of this study. The effect of a higher $\mathrm{CO}_{2}$ availability towards the isobutyrate and caproate production rates is unclear and has never been described in the literature, not even by those addressing the metabolism of E.limosum. However, the results of this study do show that $\mathrm{CO}_{2}$ consumption into biomass and/or products is not negligible, i.e. 
$15 \mathrm{mmole} /$ day. It may be of use to investigate whether increasing $\mathrm{CO}_{2}$ availability could further stimulate isobutyrate and caproate production rates. Another possible explanation for the improved production rates could be the different composition of the reactor microbiomes, which could not be further evaluated based on the currently available information. Phase IV was operated for only 7 days and the ethanol was fed again to the bioreactor to prevent potential wash-out of the ethanol chain-elongating microorganisms.

\subsubsection{Isobutyrate production continued even without feeding butyrate}

In Phase V, the operational condition was the same as Phase I, but the production and consumption profile was very different from what was observed in that earlier phase (Figure 5.2a and Figure 5.5). The most interesting finding in Phase V is that isobutyrate production continued even after the butyrate feeding was stopped and the broth butyrate concentration (around $175 \mathrm{mMC}$ ) was lower than that in Phase I (around $200 \mathrm{mMC}$ ). Previously, isobutyrate production only occurred in the presence of a high butyrate concentration in the environment, e.g. $800 \mathrm{mMC}$ in Chen et al. (2016) ${ }^{91}$ and in Phase II of this study, and 600 mMC in Phase III of this study. In Phase I, isobutyrate was not observed, even with the same operational condition used in Phase $\mathrm{V}$ and with a higher broth butyrate concentration. A possible explanation as to the isobutyrate production is the different compositions of the microbial community. In other words, the isobutyrate-producing microorganism(s) may have been enriched during Phase II-IV and continued the isomerisation/isobutyrate production with a lower butyrate concentration. For other products and substrates in Phase V (when compared with Phase I), the caproate production rate was slightly increased, while the butyrate production rate was slightly decreased. Methanol and ethanol consumption rates were slightly increased, while the acetate consumption rate increased almost 2 -fold. The increase in the acetate consumption is beneficial, as acetate can be derived from a cheap, waste-based feedstock. The overall increased substrate utilisation and production formation 


\section{Chapter 5}

rates could be attributed to the enrichment of the reactor microbiome, especially those immobilised on the polyurethane cubes $^{36}$ and/or in the biofilm attached to the inner wall of the bioreactor. This finding may be interesting for isobutyrate production in particular, as the butyrate concentration required for inducing isobutyrate production could be lowered and consequently the amount of exogenous butyrate supply could, if necessary, be reduced.

\subsubsection{Effect and role of increased $\mathrm{CO}_{2}$ feeding rate}

As discussed in section 5.3.2.3, the $\mathrm{CO}_{2}$ feeding rate in this study was 2 times higher than that of previous methanol chain elongation studies ${ }^{90,91}$. Both methanol and ethanol chainelongating microorganisms require $\mathrm{CO}_{2}$ for growth. E.limosum requires $\mathrm{CO}_{2}$ as an electron acceptor when it grows on methanol and acetate, except for one mutant ${ }^{149}$. C.kluyveri requires $\mathrm{CO}_{2}$ for protein synthesis ${ }^{150}$. It was also reported that increasing the $\mathrm{CO}_{2}$ availability in a mixed culture ethanol chain elongation increased MCFA production ${ }^{35}$. In the start-up phase, when ethanol feeding started and the ethanol consumption rate gradually increased (since day 7; Figure 5.2a), $\mathrm{CO}_{2}$ composition in the headspace gradually decreased until it was fully depleted on day 11 (Figure 5.3b). Meanwhile, methanol consumption ceased until day 24 (Figure 5.2a). From this day, when the $\mathrm{CO}_{2}$ feeding rate was increased 2-fold, methanol consumption started again (Figure 5.2a). Therefore, a sufficient $\mathrm{CO}_{2}$ supplement is possibly another essential condition, besides maintaining a stable $\mathrm{pH}$, for integrating methanol and ethanol chain elongation in one reactor system. The increased $\mathrm{CO}_{2}$ feeding rate was maintained for the rest of the operational period to prevent $\mathrm{CO}_{2}$ depletion (Figure 5.3b). Throughout the entire experiment operation, the $\mathrm{CO}_{2}$ composition in the headspace varied according to ethanol consumption. When the ethanol consumption rate increased, the headspace $\mathrm{CO}_{2}$ composition decreased due to the higher $\mathrm{CO}_{2}$ consumption (Figure 5.2a and 5.3). This was especially obvious in Phase III and Phase IV. In Phase III, the increased ethanol feeding rate stimulated the ethanol chain elongation activity and caproate production 
rate; meanwhile, the $\mathrm{CO}_{2}$ consumption increased from 10 to $20 \mathrm{mmole} /$ day and the headspace $\mathrm{CO}_{2}$ composition decreased from $20 \%$ to $10 \%$. In Phase IV, when ethanol feeding stopped, the headspace $\mathrm{CO}_{2}$ composition increased from $10 \%$ to $30 \%$. In contrast, methanol chain elongation may not require as much $\mathrm{CO}_{2}$ as ethanol chain elongation, as observed both in this study and the previous methanol chain elongation studies ${ }^{90,91}$.

\subsubsection{Methane production stimulated by methanol and inhibited by high butyrate concentration}

The methane production rate was higher in Phases I, II and V and lower in Phases III and IV (Figure 5.3 and 5.5). The highest methane production rate was up to $40 \mathrm{mmole} /$ day, which occurred on day 61. As the supplied $\mathrm{CO}_{2}$ could not sufficiently cover the total amount of carbon in the methane produced, part of the methane formation must come from other substrates. Methanol is the most probable substrate for methane production, as the methanol consumption rate increased, along with the rising methane production rate throughout the entire experiment (Figure 5.2a and 5.3a). In Phase III and IV, the lower methane production rates can be due to the presence of butyrate in high concentrations, e.g. $>8 \mathrm{~g} / \mathrm{L}$ or $363 \mathrm{mMC}$, which is inhibitory to the methanogenic activities ${ }^{137}$. In Phase I and V, butyrate concentration was always lower than $5 \mathrm{~g} / \mathrm{L}$, which might not have been enough to inhibit methanogenic activities. The previous methanol chain elongation study also had the same finding regarding the inhibition of butyrate concentration on methanogenic activity ${ }^{91}$. As mentioned in section 5.1, butyrate is a common intermediate from the hydrolysis and acidification of organic waste, and it can easily accumulate more than $8 \mathrm{~g} / \mathrm{L}^{85}$. In Chen et al. (2016), a high butyrate concentration (about $9 \mathrm{~g} / \mathrm{L}$ or $400 \mathrm{mMC}$ ) was also obtained after the hydrolysis/acidification of supermarket food waste ${ }^{91}$. Based on the induction of isobutyrate production by adding butyrate, the direct chain elongation of butyrate and ethanol (section 5.3.2.2.) and the possible inhibitory effect on methanogenic activity, using butyrate as a starting material for chain 


\section{Chapter 5}

elongation is worthy of further study. On the other hand, the methane composition in the headspace was mostly higher than $80 \%$ (Figure $5.3 \mathrm{~b}$ ), which could be considered as another value-added product ${ }^{66}$.

\subsection{Conclusion}

The concurrent use of methanol and ethanol for upgrading SCFAs was demonstrated in the present study. Without butyrate feeding, caproate and butyrate were the main products formed from chain elongation of ethanol and methanol with acetate. With a continuous butyrate feeding, caproate and isobutyrate were the main products, suggesting the concurrence of two distinct microbial pathways, i.e. ethanol and methanol chain elongation. Most likely, caproate was derived from chain elongation of ethanol and acetate, and isobutyrate from chain elongation of methanol and acetate and isomerisation of butyrate. Increasing the ethanol feeding rate further increased the caproate production rate as well as stimulating the direct use of butyrate for producing caproate via chain elongation, which improved the ethanol-to-caproate efficiency. However, in this instance, the isobutyrate production rate decreased. The reactor microbiome at the end of the experiment showed the capability to produce isobutyrate without an exogenous butyrate feeding, as well as a higher caproate production rate. Overall, this study exhibited the feasibility of stimulating two microbial pathways within the microbiome of a single reactor system for upgrading SCFAs into two value-added biochemicals simultaneously, which was done by supplying two electron donors at the same time, i.e. methanol and ethanol. 


\title{
Chapter 6. General discussion
}

\section{Benchmarking of microbial chain elongation}

\author{
based on methanol
}




\subsection{Summary of the research outcomes}

This thesis investigated the use of methanol as an alternative substrate in microbial chain elongation for chemical production from organic waste. Table 6.1 gives an overview of the main findings, in addition to the substrates used and the products achieved in each chapter. This is in response to the research questions proposed in this thesis (see Table 1.3). The most important outcomes (including the main finding) from each research chapter in this $\mathrm{PhD}$ thesis are summarised as follows:

Life Cycle Assessment of caproic acid production via microbial chain elongation (Chapter 2)

- Ethanol use has the largest share in the life-cycle environmental impact of caproic acid produced via microbial chain elongation in all assessed cases.

- Extraction solvent use also contributes substantially to the life-cycle environmental impact; however, its contribution can be reduced considerably by efficient solvent recovery, as illustrated in the pilot-scale case.

- Supermarket food waste is preferred (without applying pre-treatments) as the feedstock over the organic fraction of municipal solid waste. This is mainly due to in-situ ethanol formation in the former and the high content of hardly-biodegradable organic matter in the latter.

$\underline{\text { Methanol as an alternative electron donor for butyrate and caproate production in microbial }}$ chain elongation (Chapter 3)

- Microbial chain elongation of methanol and acetate to butyrate and caproate is feasible using an open mixed culture (proof-of-principle).

- Butyrate $(3 \mathrm{~g} / \mathrm{L})$ and caproate production $(0.1 \mathrm{~g} / \mathrm{L})$ were achieved via microbial chain elongation of methanol and acetate. 
- Continuous butyrate production $(2.3 \mathrm{~g} / \mathrm{L} ; 1.5 \mathrm{~g} / \mathrm{L}$.day $)$ from methanol and acetate was demonstrated.

$\underline{\text { Isobutyrate biosynthesis from organic waste via methanol chain elongation (Chapter 4) }}$

- Isobutyrate production via chain elongation of methanol, acetate and $\mathrm{CO}_{2}$ was demonstrated for the first time (proof-of-principle).

- Continuous isobutyrate production $(3.0 \mathrm{~g} / \mathrm{L} ; 2.0 \mathrm{~g} / \mathrm{L}$. day; $>10$ days $)$ was demonstrated using a synthetic medium containing methanol, acetate and butyrate.

- Isobutyrate $(6.2 \mathrm{~g} / \mathrm{L})$ and isovalerate $(0.5 \mathrm{~g} / \mathrm{L})$ production was achieved using acidified supermarket food waste and methanol as substrates.

- A possible mechanism for isobutyrate production via methanol chain elongation, i.e. chain elongation to butyrate and the isomerisation of butyrate, was proposed.

$\underline{\text { Concurrent use of methanol and ethanol for upgrading SCFAs into caproate and isobutyrate }}$

\section{(Chapter 5)}

- Feeding ethanol during methanol chain elongation increased the caproate production rate up to $1.5 \mathrm{~g} / \mathrm{L}$.day.

- Using butyrate as a supplement improved caproate production rate (up to 2.6 $\mathrm{g} / \mathrm{L}$. day) and induced isobutyrate production ( $1.5 \mathrm{~g} / \mathrm{L}$.day).

- Increasing the ethanol feeding rate enhanced chain elongation of butyrate to caproate. 


\section{Chapter 6}

Table 6.1. Overview of the main findings of chapters 2-5, the main substrates used, and the main products achieved (see Table 1.3 for an overview of the research questions).

\begin{tabular}{|c|c|c|c|}
\hline Chapter & Main finding & Substrate used & Achieved products \\
\hline 2 & $\begin{array}{l}\text { Ethanol use is the major environmental } \\
\text { impact source within the life cycle of } \\
\text { caproic acid production via microbial chain } \\
\text { elongation. Replacing or reducing current } \\
\text { ethanol use can be a potential strategy for } \\
\text { improving the environmental sustainability } \\
\text { of caproic acid production. }\end{array}$ & $\begin{array}{l}\text { OFMSW/SFW* } \\
\text { Ethanol }\end{array}$ & $\begin{array}{l}\text { Caproic acid (non- } \\
\text { experimental } \\
\text { result) }\end{array}$ \\
\hline 3 & $\begin{array}{l}\text { Chain elongation of methanol and acetate } \\
\text { into butyrate and caproate is feasible using } \\
\text { an open mixed culture. }\end{array}$ & $\begin{array}{l}\text { Methanol } \\
\text { Acetate }\end{array}$ & $\begin{array}{l}\text { Caproate } \\
\text { Butyrate }\end{array}$ \\
\hline 4 & $\begin{array}{l}\text { Isobutyrate production via methanol chain } \\
\text { elongation is feasible, and isobutyrate is the } \\
\text { dominant product. }\end{array}$ & $\begin{array}{l}\text { Methanol } \\
\text { Acetate/SFW }\end{array}$ & Isobutyrate \\
\hline 5 & $\begin{array}{l}\text { Concurrent use of methanol and ethanol in } \\
\text { chain elongation led to the concurrent } \\
\text { production of isobutyrate and caproate. } \\
\text { Integration of methanol chain elongation } \\
\text { into current chain elongation system is } \\
\text { possible. }\end{array}$ & $\begin{array}{l}\text { Methanol } \\
\text { Ethanol } \\
\text { Acetate } \\
\text { butyrate }\end{array}$ & $\begin{array}{l}\text { Caproate } \\
\text { Isobutyrate }\end{array}$ \\
\hline
\end{tabular}

*OFMSW: organic fraction of municipal solid waste; SFW: supermarket food waste. 


\subsection{Methanol chain elongation for caproate production: Not promising when compared with ethanol and lactate chain elongation}

Ethanol chain elongation is an established bioprocess for caproate production from organic waste. Lactate chain elongation, on the other hand, is a promising bioprocess that can also be applied to produce caproate from organic waste. Ethanol, methanol and lactate chain elongation share a potential advantage, that they can be implemented in an existing anaerobic digestion facility without the need for significant infrastructure modification. Therefore, in this section, methanol chain elongation is compared with ethanol and lactate chain elongation for the production of caproate from organic waste. There are other emerging alternative electron donors for microbial chain elongation including $\mathrm{H}_{2}$, syngas and electricity; however, the use of these alternatives requires operational facilities that are distinct from those used by ethanol chain elongation. Though these options may have potential, microbial chain elongation based on $\mathrm{H}_{2}$, syngas and electricity (bioelectrochemical chain elongation) were not included in the comparison in this section. Nevertheless, in the future, it may be of interest to include syngas and bioelectrochemical chain elongation, given the recent progresses on these processes $^{46,151}$.

When compared with ethanol chain elongation, the caproate concentration, production rate and yield of methanol chain elongation are around 30, 200 and 50 times lower, respectively. This low caproate yield in methanol chain elongation may be associated with the nature of the microbial metabolism, which balances the excessive reducing equivalent from the substrate in different ways. E.limosum is the only known bacteria that can elongate methanol and SCFAs into caproate. In the available literature, only two studies reported caproate production by E.limosum ${ }^{47,55}$, while most studies on E.limosum reported butyrate as the major product from methanol chain elongation. In Genthner et al. (1981), a pure-culture 
E.limosum growing on methanol $(50 \mathrm{mM})$ and acetate $(30 \mathrm{mM})$ produced butyrate $(8.9 \mathrm{mM}$; $0.8 \mathrm{~g} / \mathrm{L})$ and caproate $(0.1 \mathrm{mM} ; 0.01 \mathrm{~g} / \mathrm{L})^{55}$. In Lindley et al. (1987), a batch test showed that a butyrate supplement $(>100 \mathrm{mM})$ was necessary for E.limosum to produce caproate, though the caproate production was found only in trace amounts. Increasing butyrate supplement to $400 \mathrm{mM}(35.2 \mathrm{~g} / \mathrm{L})$ in the same batch test yielded $6 \mathrm{mM}$ caproate $(0.7 \mathrm{~g} / \mathrm{L})$ with $395 \mathrm{mM}$ of residual butyrate in the broth (recalculated from Fig. 1. in Lindley et al. (1987)) ${ }^{47}$. In the same study, a fed-batch fed with methanol and $\mathrm{CO}_{2}$ yielded around $10 \mathrm{mM}$ caproate $(1.2 \mathrm{~g} / \mathrm{L})$ after butyrate production accumulated to $250 \mathrm{mM}$. The caproate production reported in pureculture experiments are in the same range as the result in this thesis using an open mixed culture.

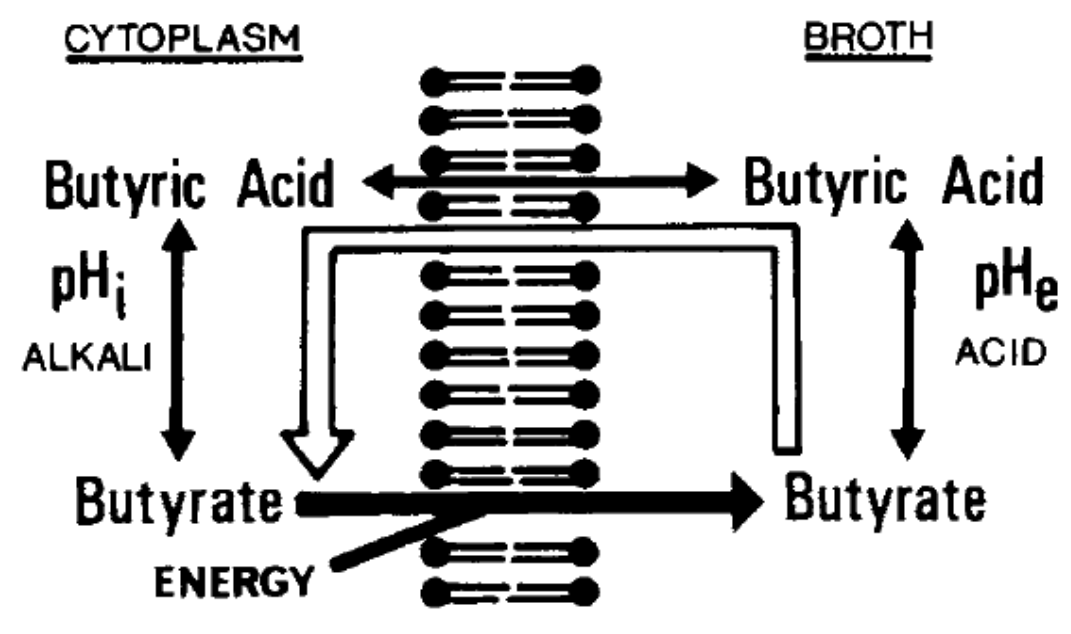

Figure 6.1. The proposed mechanism of a potential energy consuming futile cycle associated with non-passive excretion of butyrate against a concentration gradient. The figure is taken from Loubiere et al. $(1990)^{142}$. $\mathrm{pHe}$ and $\mathrm{pHi}$ refer to extracellular and intracellular $\mathrm{pH}$, respectively. Butyric acid and butyrate refer to the undisassociated and disassociated form of butyrate, respectively. 
For acetogens like C.kluyveri and E.limosum, chain elongation is a way to balance excessive reducing equivalent in the form of chain-elongated metabolites. In the case of E.limosum, an unknown compound forms alongside, or before, caproate production ${ }^{47}$. This unknown compound contains considerably more carbon and reducing equivalent flux than the caproate formed $^{47}$. It is possible that E.limosum produces another product, in addition to caproate, to store the excessive reducing equivalent from the substrate. So far the product remains unknown and requires further investigation ${ }^{47}$. It may be interesting to investigate whether the unknown compound is isobutyrate, and such an investigation could be conducted via a pure culture experiment on E.limosum.

Another possible way for E.limosum to get rid of excessive reducing equivalents is by operating an energy-consuming futile cycle that continuously excretes butyrate from the cytoplasm to the fermentation broth (see Figure 6.1$)^{142}$. This energy-consuming futile cycle keeps the intracellular butyrate concentration lower than that of broth, which helps E.limosum continue metabolising methanol and $\mathrm{CO}_{2}$ into butyrate, even when the broth butyrate concentration is at an elevated level (though not unlimitedly high) ${ }^{142}$. In either case, E.limosum shows the flexibility of using different means to balance the reducing equivalent pool. Producing caproate from methanol and butyrate seems to be one strategy, and is probably activated when broth butyrate/butyric acid concentration reaches a threshold ${ }^{47}$. The multiple and flexible reducing-equivalent balancing strategy is likely to be of survival value for E.limosum. However, it may not be of value for caproate production, due to the low caproate yield and high butyrate concentration required to trigger caproate production.

Based on the current development, methanol cannot compete with ethanol as an electron donor for caproate production via microbial chain elongation. In other words, methanol chain elongation is currently a less promising process for caproate production; though several measures can be taken to improve the process. Alternatively, methanol chain elongation can 


\section{Chapter 6}

be used to elongate acetate into butyrate prior to ethanol chain elongation producing caproate, which saves the ethanol used for chain-elongating acetate to butyrate. In that case, an additional reactor must be used, and the cost-benefit between the operation of the additional reactor and the saved ethanol should be evaluated.

When compared with lactate chain elongation, caproate concentration, production rate and total yield of methanol chain elongation are about 20, 15 and 40 times lower, respectively (see Table 6.2). It is proposed that the lactate-to-caproate process occurs via the reverse- $\beta$ oxidation, the pathway also used by the ethanol chain-elongating microorganisms. Reverse- $\beta$ oxidation seems to balance the reducing equivalent for both ethanol- and lactate-chain elongating bacteria, as no significant side products were reported (besides the propionate and valerate reported by Kucek et al. (2016), which were possibly formed via the acrylate pathway, i.e. a competing microbial process) ${ }^{43}$. This may explain why lactate chain elongation achieves a higher caproate concentration and productivity than methanol chain elongation.

Additionally, both ethanol- and lactate-chain elongation achieve a high selectivity (based on the electron balance) towards caproate, i.e. higher than $80 \%$. This is much higher than that of methanol chain elongation. In methanol chain elongation, most of the consumed carbon and energy ended up in isobutyrate, with little in butyrate and caproate (Chapter 4). Based on these comparisons, both ethanol and lactate are better electron donors than methanol for caproate production via microbial chain elongation. A possible explanation is that both ethanol- and lactate-chain elongation employ the reverse- $\beta$-oxidation as the main pathway for obtaining growth energy and managing the reducing equivalent, and excrete caproate as the main metabolite. In contrast, methanol chain-elongating bacteria may have multiple pathways, other than the reverse- $\beta$-oxidation, for managing the reducing equivalent, e.g. the 
energy-consuming futile cycle, and the multiple product output, e.g. butyrate, caproate and (possibly) the unknown compounds.

When comparing ethanol and lactate, ethanol has a better production rate and reaches a higher concentration in the continuous operation, which is the result of extensive studies on the optimisations ${ }^{30,35-37}$. In contrast, lactate chain elongation has been specifically addressed only in two studies ${ }^{43,44}$. Despite this, there is likely still room for optimisation of lactate chain elongation. From a practical perspective, acidification of the carbohydrate-rich organic waste could accumulate lactate up to $16-20 \mathrm{~g} / \mathrm{L}^{89}$, which is close to the lactate concentration range used in substrate for lactate chain elongation (i.e. $16-30 \mathrm{~g} / \mathrm{L})^{43,44}$. On the other hand, ethanol accumulation during the acidification of mixed organic waste hardly exceeded $3 \mathrm{~g} / \mathrm{L}$ in previous studies ${ }^{89}$. In this case, a substantial ethanol addition is needed for chainelongating SCFAs into caproate. Alternatively, heat pre-treatment and enzymatic hydrolysis can be applied to increase ethanol production during the acidification of mixed organic waste to up to $30 \mathrm{~g} / \mathrm{L}^{152}$, which may circumvent the additional ethanol input. However, the higher cost and environmental impact of the pre-treatment should be carefully assessed. Overall, caproate production from organic waste via lactate chain elongation can be considered a promising process worthy of further exploration, especially when a carbohydrate-rich organic waste is used as the feedstock.

Additionally, it may be interesting to integrate both ethanol- and lactate-chain elongation for caproate production. When acidifying supermarket food waste (the SFW used in Chapter 2 of this thesis) at a lower $\mathrm{pH}$, i.e. 5 , a substantial amount of lactate $(14 \mathrm{~g} / \mathrm{L}$; unpublished data) was produced along with the ethanol production $(6 \mathrm{~g} / \mathrm{L})$. The mixture of lactate and ethanol may be produced via the heterolactic fermentation of the carbohydrate fraction ${ }^{153}$ or as a consequence of diverse metabolic pathways within a mixed culture fermentation. Although $13 \mathrm{~g} / \mathrm{L}$ lactate is not as high as those employed in the lactate chain elongation 


\section{Chapter 6}

studies (i.e. 16 - $30 \mathrm{~g} / \mathrm{L}$ ), it is still a valuable substrate for caproate production. Given this context, stimulating ethanol and lactate accumulation alongside the concurrent use of lactate and ethanol for caproate production, via microbial chain elongation, in the same reactor may be interesting to explore. The concurrent use of two electron donors for chain-elongating has been demonstrated in Chapter 5 of this thesis, though this was conducted with methanol and ethanol.

In sum, based on the experimental results, methanol chain elongation is currently not a promising process for caproate production, due to its low caproate production rate and yield. Optimisation of caproate production via methanol chain elongation may be investigated, but room for improvement may be limited, possibly due to the nature of the metabolism. Nevertheless, there are many process parameters that have not been tested in methanol chain elongation. Examples include pH, HRT and biomass retention. Future studies may further explore the effectiveness of these strategies on improving caproate production via methanol chain elongation. Alternatively, methanol chain elongation may be used as an isobutyrate biosynthesis technology. In methanol chain elongation, a relatively high isobutyrate concentration and production rate were achieved, and another novel isomerised fatty acid, e.g. isovalerate, was also discovered (Chapter 4). It may be more valuable to position methanol chain elongation as a bioprocess for isomerised fatty acid production and to further explore its potential. 
Table 6.2. Overview of the caproate production and in microbial chain elongation under a non-sterile condition using different electron donors.

\begin{tabular}{|c|c|c|c|c|c|c|c|c|c|}
\hline $\begin{array}{l}\text { Electron } \\
\text { donor* }\end{array}$ & $\begin{array}{l}\text { Electron } \\
\text { acceptor }\end{array}$ & $\begin{array}{l}\text { Reactor type } \\
(+ \text { HRT) }\end{array}$ & $\mathrm{pH}$ & $\begin{array}{l}\text { Temper } \\
\text { ature } \\
\left({ }^{\circ} \mathrm{C}\right)\end{array}$ & $\begin{array}{l}\text { Caproate } \\
\text { concentra } \\
\text { tion }(\mathrm{g} / \mathrm{L})\end{array}$ & $\begin{array}{l}\text { Caproate } \\
\text { productio } \\
\text { n rate } \\
\text { (g/L.day) }\end{array}$ & $\begin{array}{l}\text { Yield } \\
\text { (gCOD } \mathrm{g}_{\text {cap }} \\
\text { roate } / \mathrm{gCO} \\
\mathrm{D}_{\text {substrate }} \text { ) }\end{array}$ & $\begin{array}{l}\text { Other } \\
\text { products }\end{array}$ & Source \\
\hline $\begin{array}{l}\text { Ethanol } \\
(13.8 \mathrm{~g} / \mathrm{L})\end{array}$ & $\begin{array}{l}\text { Acetate } \\
(7.2 \mathrm{~g} / \mathrm{L})\end{array}$ & $\begin{array}{l}\text { Upflow } \\
\text { anaerobic filter } \\
(\text { HRT }=0.2 \text { day; } \\
\text { Day 69) }\end{array}$ & $\begin{array}{l}6.5- \\
7.2\end{array}$ & 30 & 9.3 & 46.5 & 0.49 & $\begin{array}{l}\text { Caprylate } \\
(0.3 \mathrm{~g} / \mathrm{L})\end{array}$ & $\begin{array}{l}\text { Grootsc } \\
\text { holten } \\
\text { et } \\
\text { al.,201 } \\
3^{35}\end{array}$ \\
\hline $\begin{array}{l}\text { Lactate } \\
(30 \mathrm{~g} / \mathrm{L})\end{array}$ & NA & Batch & $\begin{array}{l}6.0- \\
6.5\end{array}$ & 30 & 12.5 & 2.5 & 0.86 & NA & $\begin{array}{l}\text { Zhu et } \\
\text { al., } \\
2015^{44}\end{array}$ \\
\hline $\begin{array}{l}\text { Lactate } \\
(20 \mathrm{~g} / \mathrm{L})\end{array}$ & NA & Fed-batch & $\begin{array}{l}6.0- \\
6.5\end{array}$ & 30 & 23.4 & NA & NA & NA & $\begin{array}{l}\text { Zhu et } \\
\text { al., } \\
2015^{44}\end{array}$ \\
\hline $\begin{array}{l}\text { Lactate } \\
(16.2 \mathrm{~g} / \mathrm{L})\end{array}$ & $\begin{array}{l}\text { Butyrate } \\
(8.9 \mathrm{~g} / \mathrm{L})\end{array}$ & $\begin{array}{l}\text { Upflow } \\
\text { anaerobic filter } \\
(\text { HRT = } 1.9 \text { day; } \\
\text { Day } 176-193)\end{array}$ & $\begin{array}{l}5.0- \\
5.5\end{array}$ & 34 & 5.9 & 3.1 & 0.39 & NA & $\begin{array}{l}\text { Kucek } \\
\text { et al, } \\
2016^{43}\end{array}$ \\
\hline $\begin{array}{l}\text { Methanol } \\
(6.0 \mathrm{~g} / \mathrm{L})\end{array}$ & $\begin{array}{l}\text { Acetate } \\
(3.2 \mathrm{~g} / \mathrm{L}) \\
\text { Butyrate } \\
(9.1 \mathrm{~g} / \mathrm{L})\end{array}$ & $\begin{array}{l}\text { Upflow } \\
\text { anaerobic filter } \\
(\text { HRT }=1.7 \text { day; } \\
\text { Day } 54 \text { in } \\
\text { Chapter } 4)\end{array}$ & $\begin{array}{l}6.0- \\
6.5\end{array}$ & 35 & 0.3 & 0.2 & 0.01 & $\begin{array}{l}\text { Isobutyrate } \\
(3 \mathrm{~g} / \mathrm{L})\end{array}$ & $\begin{array}{l}\text { Chen et } \\
\text { al, } \\
2016^{91}\end{array}$ \\
\hline $\begin{array}{l}\text { Methanol } \\
(6.9 \mathrm{~g} / \mathrm{L})\end{array}$ & $\begin{array}{l}\text { SCFAs } \\
\text { (from } \\
\text { acidified } \\
\text { SFW) }\end{array}$ & Batch & 5.9 & 35 & 0.8 & NA & 0.04 & $\begin{array}{l}\text { Isobutyrate } \\
(6.2 \mathrm{~g} / \mathrm{L}) \\
\text { Isovalerate } \\
(0.5 \mathrm{~g} / \mathrm{L})\end{array}$ & $\begin{array}{l}\text { Chen et } \\
\text { al, } \\
2016^{91}\end{array}$ \\
\hline $\begin{array}{l}\text { Ethanol } \\
\text { (from a } \\
\text { syngas } \\
\text { fermentati } \\
\text { on } \\
\text { effluent) }\end{array}$ & $\begin{array}{l}\text { Acetate } \\
\text { (from a } \\
\text { syngas } \\
\text { fermentati } \\
\text { on } \\
\text { effluent) }\end{array}$ & $\begin{array}{l}\text { Upflow } \\
\text { anaerobic filter } \\
\text { with in-line } \\
\text { extraction (Phase } \\
\text { II-Period } 11 \text {; day } \\
\text { 174-186; HRT = } \\
1.5 \text { day) }\end{array}$ & 5.2 & 30 & $0.03^{\dagger}$ & $5^{t}$ & 0.11 & $\begin{array}{l}\text { Caprylate } \\
(0.7 \mathrm{~g} / \mathrm{L})\end{array}$ & $\begin{array}{l}\text { Kucek } \\
\text { et al., } \\
2016^{154}\end{array}$ \\
\hline
\end{tabular}

Note: Selectivity was not included, as different units and calculations for selectivity were used. It is not comparable and too difficult to be recalculated.

*Electrode can also be used to provide electrons directly to microorganisms for chain elongation, i.e. a bioelectrochemical system. Such process was not included in this comparison table considering the distinct process configurations.

${ }^{\dagger}$ This refers to the residual broth caproate concentration after the in-line extraction.

\$The production rate was taken from Kucek et al. (2016), which included both the residual broth caproate and the extracted caproate production. 


\subsection{Methanol chain elongation for isomerised fatty acid production: a promising and novel process, but improvement is needed}

In Chapter 4, isobutyrate production via methanol chain elongation was compared with other bio-based isobutyrate production processes (see Chapter 4, Table 4.3). It was shown that methanol chain elongation is a promising isobutyrate production process, mainly due to the use of a self-regenerated biocatalyst, the circumvention of the sterilisation process, the use of mixed organic waste as substrate and the demonstrated feasibility of a continuous operation. In this section, we elaborate on the potential and promise of isomerised fatty acids as novel chain elongation products. Three aspects were selected to be addressed in this elaboration; namely production performance, market potential (including price and applications) and the possible integration with the current chain elongation plant.

\subsubsection{Production performances}

In this section, we first compare the isobutyrate and isovalerate production in methanol chain elongation with other potential novel products in chain elongation, i.e. caprylate and odd-chain fatty acids including valerate and heptanoate. Table 6.3 summarises the production performances of the potential products in microbial chain elongation, with the exception of caproate which was evaluated in section 6.2. Isobutyrate production using acidified SFW and methanol shows the highest yield and concentration, especially the latter, which is 2-30 times higher than the other products. This is due to the fact that, in most of the chain elongation studies, reverse- $\beta$-oxidation producing caproate is the most dominant process due to the use of ethanol or lactate as the electron donor. In Grootscholten et al. (2013), although higher heptanoate and valerate concentrations were achieved due to the propionate supplement, caproate concentration was still the highest amongst all products ${ }^{71}$. Kucek et al. (2016) reported an improved caprylate production in chain elongation by employing a higher 
substrate ethanol-acetate ratio and an in-line MCFA extraction ${ }^{154}$. Nevertheless, the caproate concentration is still in the same range of that of caprylate. In contrast, during methanol chain elongation, isobutyrate is the dominant product, and its concentration and production rate is always 10 -fold of the caproate. This makes isobutyrate an interesting product of methanol chain elongation, because of the absence of the caproate dominance in the products. When comparing the production rate, isobutyrate is 2-3 times lower than that of other products. As this thesis is the proof-of-concept, there may still be room for improvements.

The main drawback of isobutyrate production via methanol chain elongation is the high butyrate concentration required. There is a possible improvement strategy based on our hypothesis of isobutyrate production mechanism (Chapter 4, Figure 4.5). In our hypothesis, methanol chain elongation continues producing butyrate. The high and persistent butyrate concentration triggers the isomerisation of butyrate, possibly to overcome the butyrate toxicity. As illustrated in Figure 6.1, the main butyrate toxicity possibly originates from the protonated form of butyrate, i.e. butyric acid. Butyric acid can passively diffuse through the cell membrane, and extra energy is invested to excrete the intracellular butyrate which resulted from the passive diffusion of butyric acid. The broth butyric acid concentration, instead of the broth butyrate concentration, may be the actual trigger for the isobutyrate production. In this case, maintaining a more acidic $\mathrm{pH}$ could be of use when trying to stimulate isobutyrate production. This is because the ratio between butyric acid and butyrate increases with decreasing $\mathrm{pH}$. 
Table 6.3. Overview of the other value-added products, in addition to caproate, chain elongation using different electron donors.

\begin{tabular}{|c|c|c|c|c|c|c|c|c|c|}
\hline $\begin{array}{l}\text { Electron } \\
\text { donor }\end{array}$ & $\begin{array}{l}\text { Electron } \\
\text { acceptor }\end{array}$ & $\begin{array}{l}\text { Reactor type } \\
(+\mathrm{HRT})\end{array}$ & $\mathrm{pH}$ & $\begin{array}{l}\text { Temper } \\
\text { ature } \\
\left({ }^{\circ} \mathrm{C}\right)\end{array}$ & $\begin{array}{l}\text { Novel } \\
\text { product }\end{array}$ & $\begin{array}{l}\text { Concentr } \\
\text { ation } \\
(\mathrm{g} / \mathrm{L})\end{array}$ & $\begin{array}{l}\text { Production } \\
\text { rate } \\
(\mathrm{g} / \mathrm{L} . \mathrm{day})\end{array}$ & $\begin{array}{l}\begin{array}{l}\text { Yield } \\
(\mathrm{gCOD} /\end{array} \\
\mathrm{gCOD}_{\text {su }} \\
\text { bstrate })\end{array}$ & Source \\
\hline $\begin{array}{l}\text { Ethanol } \\
(13.8 \mathrm{~g} / \mathrm{L})\end{array}$ & $\begin{array}{l}\text { Acetate }(7.2 \\
\mathrm{g} / \mathrm{L})\end{array}$ & $\begin{array}{l}\text { Upflow } \\
\text { anaerobic } \\
\text { filter }(\mathrm{HRT}= \\
0.2 \text { day; Day } \\
69)\end{array}$ & $\begin{array}{l}6.5- \\
7.2\end{array}$ & 30 & Caprylate & 0.3 & 1.8 & 0.02 & $\begin{array}{l}\text { Grootscho } \\
\text { lten et al., } \\
2013^{35}\end{array}$ \\
\hline
\end{tabular}

\begin{tabular}{|c|c|c|c|c|c|c|c|c|c|}
\hline $\begin{array}{l}\text { Ethanol } \\
(13.7 \mathrm{~g} / \mathrm{L})\end{array}$ & $\begin{array}{l}\text { Acetate- }(0.6 \\
\mathrm{g} / \mathrm{L}) \\
\text { Propionate } \\
(7.3 \mathrm{~g} / \mathrm{L})\end{array}$ & $\begin{array}{l}\text { Upflow } \\
\text { anaerobic } \\
\text { filter (HRT = } \\
0.7 \text { day; Phase }\end{array}$ & $\begin{array}{l}6.5- \\
7.0\end{array}$ & 30 & Heptanoate & 3.2 & 4.5 & 0.19 & $\begin{array}{l}\text { Grootscho } \\
\text { lten et al, } \\
2013^{71}\end{array}$ \\
\hline
\end{tabular}

$\begin{array}{llll}\text { Valerate } \quad 4.6 & 6.6 & 0.23\end{array}$

\begin{tabular}{|c|c|c|c|c|c|c|c|c|c|}
\hline $\begin{array}{l}\text { Methanol } \\
(6.0 \mathrm{~g} / \mathrm{L})\end{array}$ & $\begin{array}{l}\text { Acetate }(3.2 \\
\text { g/L) } \\
\text { Butyrate }(9.1 \\
\mathrm{g} / \mathrm{L})\end{array}$ & $\begin{array}{l}\text { Upflow } \\
\text { anaerobic } \\
\text { filter (HRT = } \\
1.7 \text { day; Day } \\
53 \text { in Chapter } \\
\text { 4) }\end{array}$ & $\begin{array}{l}6.0- \\
6.5\end{array}$ & 35 & Isobutyrate & 3 & 2 & 0.11 & $\begin{array}{l}\text { Chen et } \\
\text { al.,2016 }\end{array}$ \\
\hline \multirow[t]{2}{*}{$\begin{array}{l}\text { Methanol } \\
(6.9 \mathrm{~g} / \mathrm{L})\end{array}$} & $\begin{array}{l}\text { Acidified } \\
\text { SFW }\end{array}$ & Batch & 5.9 & 35 & Isobutyrate & 6.2 & - & 0.27 & $\begin{array}{l}\text { Chen et } \\
a l, .2016^{90}\end{array}$ \\
\hline & & & & & Isovalerate & 0.5 & - & 0.02 & \\
\hline $\begin{array}{l}\text { Ethanol } \\
(42.5 \mathrm{~g} / \mathrm{L} \\
\text { from a } \\
\text { syngas } \\
\text { fermentati } \\
\text { on } \\
\text { effluent })\end{array}$ & $\begin{array}{l}\text { Acetate (6.6 } \\
\text { g/L from a } \\
\text { syngas } \\
\text { fermentation } \\
\text { effluent) }\end{array}$ & $\begin{array}{l}\text { Upflow } \\
\text { anaerobic } \\
\text { filter with in- } \\
\text { line extraction } \\
\text { (Phase II- } \\
\text { Period } 11 \text {; day } \\
174-186 \text {; HRT } \\
=1.5 \text { day) }\end{array}$ & 5.2 & 30 & Caprylate & $0.7^{\dagger}$ & $5.4^{+}$ & 0.21 & $\begin{array}{l}\text { Kucek et } \\
a l, .2016^{154}\end{array}$ \\
\hline
\end{tabular}

Note: Selectivity was not included, as different units and calculations for selectivity were used. It is not comparable and difficult to be recalculated.

${ }^{\dagger}$ This refers to the residual broth concentration after the in-line extraction.

*The production rate was taken from Kucek et al. (2016), which included both the residual broth caprylate and the extracted caprylate production. 
The experimental result of Chapter 4 also supported this hypothesis regarding the effect of pH. Figure 6.2 gives an overview of isobutyrate, butyrate, butyric acid (calculated) and total butyrate concentration (sum of butyrate and butyric acid) for the continuous (Figure 6.2a) and batch experiments (Figure 6.2b) in Chapter 4. In Figure 6.2a (Phase V), the butyrate and total butyrate concentration are about 2 times higher than in Figure 6.2b. However, the isobutyrate concentration in Figure 6.2a is lower than in Figure 6.2b. This shows that a higher butyrate concentration is not necessarily beneficial when attempting to achieve a higher isobutyrate concentration. Instead, the butyric acid concentration in the broth may be the key factor for stimulating isobutyrate production. A lower $\mathrm{pH}$ can result in a higher butyric acid concentration without a butyrate concentration as high as that shown in Chapter 4, which may be useful for stimulating a higher isobutyrate production. For readers' information, the $\mathrm{pH}$ for the continuous experiment was around 6.2 - 6.5 and for the batch experiment 5.9, as reported in Chapter 4. In Figure 6.2b, the isobutyrate concentration rises with the increased broth butyric acid concentration. This provides another potential support to our hypothesis regarding the effect of $\mathrm{pH}$. However, the experiments conducted in Chapter 4 were not specifically designed to investigate the effect of $\mathrm{pH}$ on isobutyrate production, so further study is necessary in order to justify this hypothesis. Interestingly, isovalerate was formed in the batch experiment in Chapter 4, where a higher valerate concentration and a lower $\mathrm{pH}$ were present. It would be interesting to investigate whether the isovalerate production can be further stimulated by increasing the broth valeric acid concentration. 

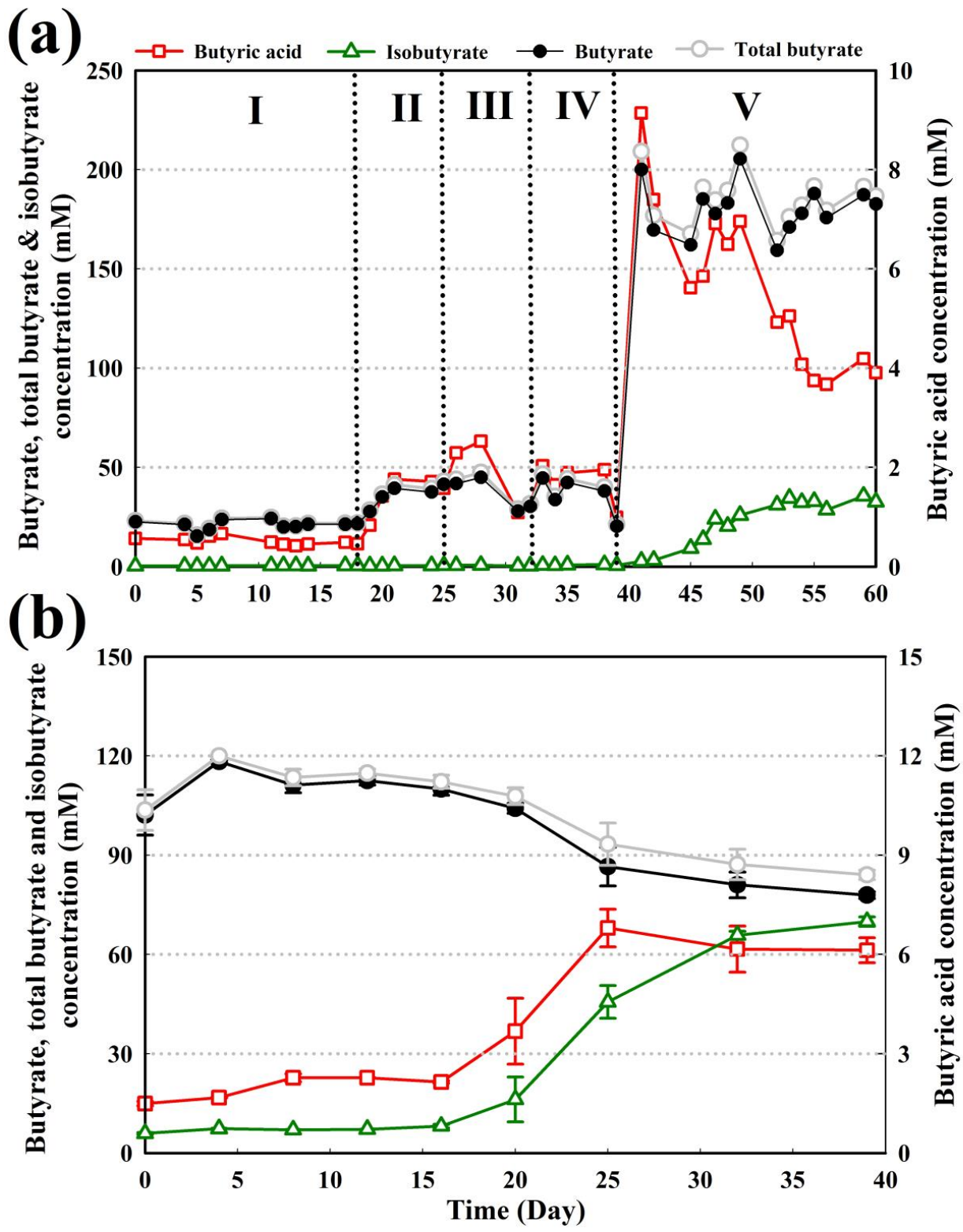

Figure 6.2. The overview of isobutyrate, butyrate, butyric acid (the undisassociated form of butyrate; calculated based on the broth $\mathrm{pH}$ and butyrate concentration) and total butyrate concentration (sum of butyrate and butyric acid) for the continuous (a) and batch experiment (b) in Chapter 4. Refer to Chapter 4 for the detailed experimental and phase conditions (Phase I-V). 


\subsubsection{Applications, market potentials and prices}

Isobutyrate is a bulk chemical with a higher price than butyrate, though it has a similar price as caproate, based on the price per mole carbon (see Table 1.2). Isobutyrate, however, has more extensive applications than caproate, because it is an existing platform chemical. A promising application is a precursor for isobutanol. Isobutanol has been considered a better fuel additive than ethanol due to its property of being a drop-in fuel, its high energy content and its higher octane number ${ }^{127}$. USA-based biofuel company Gevo (Minnesota, USA), is commercialising the isobutanol production via fermentation of glucose using a metabolically engineered microorganism as the biocatalyst (http://www.gevo.com/) ${ }^{126}$. They estimate the annual market potential for isobutanol as being more than 450 million tonnes, when targeting the use of isobutanol as a fuel. It was demonstrated that syngas can be used to reduce isobutyrate into isobutanol via a pure-culture fermentation ${ }^{124}$. This syngas fermentation can be potentially integrated with methanol chain elongation to produce isobutanol from two types of sustainable feedstock; lignocellulosic waste and mixed organic waste (see section 1.3 for the definition of a mixed organic waste). The advantage of isobutanol via methanol and syngas fermentation, comparing with the Gevo process, includes the use of a sustainable feedstock and the use of a self-regenerating biocatalyst, both of which makes the process potentially more environmentally sustainable and cost-effective. This makes isobutanol an interesting application for isobutyrate produced via methanol chain elongation, especially considering the growing market demand for isobutanol.

Another promising application of isobutyrate is as the precursor for methacrylate. Methacrylate is the basic building block for methyl methacrylate (MMA). The polymer of MMA is called polymethylmethacrylic acid (PMMA), a transparent thermoplastic. MMA has a large market size of 2.2 million tonnes per year, globally ${ }^{57}$. The production of MMA currently uses a fossil-based feedstock, thereby contributing 20 million tonnes of global warming potential $\left(\mathrm{CO}_{2}\right.$-eq) annually ${ }^{155}$. To obtain MMA from organic waste may therefore 


\section{Chapter 6}

be more environmentally sustainable than existing MMA production procedures. Using an oxidative dehydrogenation process, isobutyrate can be converted into methacrylate, which is then esterified with methanol to PMMA ${ }^{156}$. The oxidation of isobutyrate to methacrylate was also shown to be feasible via microbial process, though only using a pure culture ${ }^{157}$. Given this context, it may be interesting to study the feasibility of adding a post-methanol chain elongation aerobic process to convert isobutyrate into methacrylate.

A possible obstacle for the application of isobutyrate is the process of separating it from the fermentation broth. To the author's best knowledge, there are no studies which address isobutyrate separation from fermentation broths, possibly because isobutyrate has not been reported as a major fermentation product. Isobutyric acid has a solubility of $16.7 \mathrm{~g} / \mathrm{L}$ in water and a logarithmic acid dissociation constant (pKa) of 4.84 at $20^{\circ} \mathrm{C}^{158,159}$, which is similar to butyrate. Solvent extraction for separating isobutyrate may be a feasible practice, as this process has been used for separating butyrate from fermentation broths in prior studies ${ }^{160,161}$. Other example techniques used for separating organic acids from fermentation broth include, for example, precipitation ${ }^{22}$ and electrodialysis ${ }^{85}$. For a fermentation broth containing both SCFAs and MCFAs, precipitation has a tendency to separate mainly MCFAs from the fermentation broth $^{22}$. Electrodialysis, on the other hand, separates smaller SCFAs like acetate and propionate prior to longer chain fatty acids, e.g. butyrate and MCFAs ${ }^{85}$. Neither of these may be effective techniques for isobutyrate separation. Further study should address the separation of isobutyrate or its derivatives, e.g. methacrylate, from the fermentation broth using solvent extraction or other emerging techniques.

Overall, isobutyrate has a large, established market thanks to its variable applications. Isobutyrate and its derivatives are mostly produced from fossil-based feedstock. Substituting the fossil-based isobutyrate and its derivatives with the isobutyrate produced from organic waste is therefore worthy of further study. 


\subsubsection{Integration of isobutyrate production to the current chain elongation production chain}

In Chapter 5, we demonstrated the concurrent use of methanol and ethanol in chain elongation to produce caproate and isobutyrate in a single reactor. Although the isobutyrate production rate needs further improvement, such demonstration, as shown in Chapter 5, already implies the possibility of integrating isobutyrate production into the existing chain elongation production system. Due to the different solubility between caproate $(10.8 \mathrm{~g} / \mathrm{L})$ and isobutyrate (miscible) in water, it may be possible to selectively extract caproate from the broth via solvent extraction, depending on the diffusion coeffiicent of the solvetn towards different organic acids. In the case that the isobutyrate-to-butyrate ratio can be improved significantly (as discussed in section 6.3.2.), the residual broth will contain mainly isobutyrate. The residual broth containing isobutyrate may be used for other applications as discussed in section 6.3.2. Another possible integration is to use the residual fermentation broth from the current chain elongation production system as a feedstock for isobutyrate production. The current residual fermentation broth, after caproate extraction, contains a large amount of SCFAs, an ideal feedstock for isobutyrate production. By adding another fermentation process with methanol for isobutyrate production, the residual fermentation broth is valorised. In this way, the overall cost-effectiveness and environmental performance of the chain elongation production system may be improved due to the multiple output products.

\subsection{Chain elongation and organic waste management: A life- cycle perspective}

This thesis deals with managing mixed organic waste with the recovery of value-added chemicals. One could question, however, whether this is a better way to treat organic waste than other existing practices. Current waste management strategies are landfilling, 


\section{Chapter 6}

incineration, composting and anaerobic digestion. An emerging type of waste management recovers value-added materials, i.e. polyhydroxyalkanoate (PHA) production. These methods have their specific pros and cons as well as their own specific niche within waste management. The preferred method is dependent on many factors including waste composition and availability, economic feasibility, etc. In this section, microbial chain elongation is compared with other waste management practices. Based on this, the author discusses how microbial chain elongation could evolve into a more effective and efficient waste management strategy. One should note, however, that the proceeding discussion does not try to justify the superiority of microbial chain elongation over other waste management strategies. Rather, the author intends to discuss how to promote the use of microbial chain elongation within the waste management industry, and vice versa, i.e. how microbial chain elongation can improve the current waste management.

\subsubsection{Pros and cons when comparing microbial chain elongation with other waste management strategies}

A major advantage of microbial chain elongation over other waste management strategies is the higher economic value of its end-products, i.e. caproate and isobutyrate. Caproic acid has a market price ranging from $2.2-8.2 € / \mathrm{kg}$ and isobutyrate has a market price of around $1.9 € / \mathrm{kg}$ (see Table $1.1^{\mathrm{a}}$ ). Both seem higher than that of PHA. For PHA, in order to compete with petroleum-based plastic, the price should be below $1.4 € / \mathrm{kg}^{162}$. The production costs of PHA are estimated to be $0.4^{163}-1.4 € / \mathrm{kg}^{164}$, depending on the carbon source and the bioprocess employed. This gives a limited profit margin for PHA production. The higher economic value of caproate and isobutyrate may provide a larger profit margin, which makes microbial chain elongation more economically attractive. Waste incineration, anaerobic digestion and composting typically yield energy, energy carriers and soil amendments.

\footnotetext{
${ }^{\text {a }}$ Considering the density of caproate is around $0.93 \mathrm{~kg} / \mathrm{L}$
} 
However, they are usually less economically competitive, as discussed in the introduction of this thesis. It may be of use to perform a comparative economic analysis to validate the economic advantage of microbial chain elongation.

Another major advantage of microbial chain elongation over other waste management strategies is the potentially higher carbon recovery efficiency in its end products. During landfilling, incineration and composting, most of the carbon in the waste is transformed into $\mathrm{CO}_{2}$. In the case of microbial chain elongation, theoretically, three-quarters of carbon in the waste is recovered in the form of caproic acid, which is a higher recovery rate than that of anaerobic digestion and PHA production (see Table 1.1). The higher carbon recovery efficiency makes microbial chain elongation a more efficient resource-recovering waste management strategy over the others.

A potential issue for microbial chain elongation is that it cannot be directly applied to manage all kinds of waste, due to its limited feedstock spectrum. Microbial chain elongation is not an option for the treatment of hardly-biodegradable waste, like plastics and polymers. Integration with incineration or landfilling is therefore necessary to completely manage the waste. This is the same for the other biological waste treatment processes, i.e. composting, anaerobic digestion and PHA production. Thus, the waste management processes are not completely mutually exclusive. Therefore, exploring the variety of waste strategy combinations currently available should be addressed. It is important to integrate the current waste management practice with the emerging resource-recovering waste treatment (e.g. microbial chain elongation and PHA production) to maximise the resource-recovery efficiency and cost-effectiveness of waste management, as well as to minimise the environmental impacts generated throughout the waste management chain. A life-cycle approach could be employed when analysing such integration. 


\section{Chapter 6}

Overall, microbial chain elongation is a promising waste management strategy because of its higher economic feasibility, higher carbon recovery efficiency and continuous up-scaling. Although microbial chain elongation cannot be a comprehensive waste management strategy by itself, the expansion of its feedstock spectrum is beneficial as it increases its applicability and role in an integrated waste management scheme. In 6.4.2, the feedstock expansion for microbial chain elongation during this thesis and in the future is discussed.

\subsubsection{Expanding the feedstock spectrum: lignocellulose, and what next?}

In this thesis, lignocellulosic biowaste was integrated into the feedstock spectrum of microbial chain elongation via the use of methanol. Such integration not only promotes the application of microbial chain elongation in waste management, but also formulates a new biorefinery chain by integrating the thermochemical and biochemical conversion processes. The integration of thermochemical process has the potential to further expand the feedstock spectrum of microbial chain elongation beyond the mixed organic waste and lignocellulosic waste that were the focus throughout this thesis. In recent years, the use of thermochemical conversion processes to treat non-recyclable plastic waste has emerged ${ }^{165}$. The cogasification of dry biomass and plastic waste for high-quality syngas that may be directly used for methanol production was demonstrated, though this is still in the early stages of development ${ }^{166}$. Given this wider context, plastic waste may become an interesting and promising feedstock for chemical production via methanol chain elongation. In such a case, one can expect that the feedstock for microbial chain elongation will increase substantially. Subsequently, novel products and applications of the end-products from microbial chain elongation will have to be further explored to balance the feedstock supply and the market demand for the end-product from microbial chain elongation. 


\subsubsection{Food, fuel or material? Decision making on what to be produced via microbial chain elongation}

What to produce from organic waste via microbial chain elongation will likely become an important question in the future. Unlike current waste management practices, microbial chain elongation opens the door to valorising mixed organic waste into functional materials, rather than only energy or energy carriers (e.g. $\mathrm{CH}_{4}$ ). More novel products, and their application, produced through microbial chain elongation are also emerging (this thesis) and expanding ${ }^{33}$. Given this context, the decision making process regarding what to produce from waste may become more complex.

Caproate produced from organic waste via microbial chain elongation is about to be used as a feed additive to improve the feed-to-food conversion efficiency in the animal production industry. This application is beneficial in terms of increasing food supply and production efficiency. On the other hand, caproate can also be used as a precursor for fuels and lubricants, which are currently manufactured from fossil-based resources. Using caproate for fuel and lubricant production can be beneficial in terms of reducing (1) the dependency on fossil-based resources and (2) the associated environmental impacts. One could question what, in the end, is more sustainable: promoting animal production or produing renewable fuels from organic waste. Questions like this can only be answered by accounting for the environmental, economic and social sustainability of the wider system, meaning a continuation of the LCA performed in this thesis is important. Further analyses could, for instance, try to assess multiple applications of caproate and the consequences of these applications from an environmental, economic and social sustainability point of view. Such analyses can help decision making in promoting the effectiveness of microbial chain elongation for waste management. 


\subsubsection{Altering the current waste separation scheme to improve the valorisation of OFMSW}

Valorisation of OFMSW via microbial chain elongation needs to be further improved. OFMSW is a mixed organic waste continuously produced in a large quantity and needs to be disposed of. Treating OFMSW with microbial chain elongation was shown to be less effective when compared with other mixed organic waste like supermarket food waste and food processing waste, probably due to the high lignocellulosic content of OFMSW (in some cases reaching up to $90 \%)^{39}$. A thermochemical conversion process, e.g. gasification of OFMWS, is unlikely to be energy-efficient due to the high water content of the waste. Moreover, comparing with other mixed organic wastes, the prevention and reduction of OFMSW generation is likely to be more difficult due to the involvement of greater numbers of stakeholders.

New waste separation schemes based on the water and lignocellulosic content may be useful to improve the use of OFMSW in microbial chain elongation, provided that a thermochemical conversion process is integrated into microbial chain elongation. In the new waste separation scheme, dry organic waste like lignocellulosic waste and plastics can be collected together, and the wet and readily biodegradable organic waste, like kitchen waste, can be collected together. The dry organic waste can be processed via the thermochemical conversion process to produce syngas, which can be then used to produce methanol (via a chemical process) or ethanol (e.g. via syngas fermentation). The wet fraction can be used directly in the hydrolysis and acidification for SCFA production, which is expected to have a higher conversion efficiency (due to the smaller quantity of hardly-biodegradable organic matter) and an accumulation of more reduced intermediates, e.g. ethanol and lactate. This can be described as a resource-recovery-oriented waste separation scheme, which may be useful for improving the resource recovery efficiency of OFMSW. 
There are potential drawbacks that must be overcome and evaluated in the new waste separation scheme, such as the potentially low social acceptance. A more detailed evaluation should be performed to confirm its feasibility and the benefits to the current waste management practice of OFMSW. The proposal of this new waste separation scheme did not aim to adapt the current waste management scheme to promote microbial chain elongation. Instead, through this proposal, the author aims to initiate a reflection on the current waste management scheme and how it can be altered to become a resource-recovery-oriented waste management system.

\subsection{Concluding remarks \& Outlook}

Microbial chain elongation has been considered a promising and serious bioprocess for several years. Through my thesis research, microbial chain elongation was further developed. Ethanol use and the limited end-product spectrum were identified as the potential bottlenecks for promoting chain elongation in waste management. Methanol was used to replace ethanol in microbial chain elongation for caproate production. Production of a new product, i.e. isobutyrate, in methanol chain elongation was achieved. Moreover, the integration of methanol and ethanol chain elongation was demonstrated. My thesis shows that microbial chain elongation based on methanol can be considered a promising bioprocess, especially when used for isobutyrate production. The use of methanol also introduces the thermochemical conversion process into microbial chain elongation, which broadens the feedstock spectrum for caproate and isobutyrate production. Nevertheless, further research is still required, especially focusing on improving the isobutyrate production performance and integrating microbial chain elongation with other existing waste management practices. Creating and facilitating a discussion on the application of the products of microbial chain elongation would be greatly beneficial. Based on the application, a complete life cycle assessment, i.e. cradle-to-grave or even cradle-to-cradle, can be performed to assess the 
Chapter 6

overall environmental impacts of microbial chain elongation. When comparing with existing waste management practices, microbial chain elongation can recover end-products with higher economic values from organic waste, which makes this process more economically attractive. It is recommended to make an economic analysis on microbial chain elongation, in comparison with other waste management methods, in the near future to further evaluate its cost-effectiveness. 
Summary 
Our society relies heavily on fossil resources to fulfill our energy and commodity demands and this dependence has led to negative economic, environmental and societal consequences. The re-generation rate of fossil resources is much slower than their consumption rate, making these resources a non-renewable feedstock for the supply of energy and goods to our society. Moreover, the rapid consumption of fossil resources releases the carbon sequestrated in the last few million years in a much shorter time span, which contributes to the carbon dioxide $\left(\mathrm{CO}_{2}\right)$ concentration increase in the atmosphere and potentially global warming. The geographically-uneven distribution of fossil resources also induces social insecurities and political conflicts. An alternative feedstock is necessary for energy and goods supply to our society, and such alternative feedstock should be renewable, economically sustainable, environmentally sound and geographically wide-spread,.

Organic waste is an emerging and promising alternative feedstock. The production of organic waste is inevitable, occurs in large quantities and is geographically wide-spread, especially the so-called "mixed organic waste," e.g. organic fraction of municipal solid waste (OFMSW) and food processing waste. Mixed organic waste contains a large quantity of carbon materials that can be valorised into energy carriers and commodities. However, the extremely heterogeneous composition and the relatively high water content of mixed organic waste make its valorisation via the current waste management methods (e.g. incineration, composting and anaerobic digestion) less efficient and not economically attractive. Given this context, a novel bioprocess based on a mixed culture fermentation, i.e. microbial chain elongation, was developed to promote the valorisation of mixed organic waste. In microbial chain elongation, the diverse, complex organic matter in mixed organic waste are homogenised via hydrolysis and bacterial acidification into basic building blocks; like short chain fatty acids (SCFAs), $\mathrm{CO}_{2}$ and hydrogen $\left(\mathrm{H}_{2}\right)$. After the homogenisation, energy-rich co-substrates like ethanol are added to these basic building blocks to synthesise medium 
chain fatty acids (MCFAs) via a mixed culture fermentation. MCFAs are organic compounds with a higher economic value and a higher energy content. Microbial chain elongation can be operated under a non-sterile condition, which makes it applicable to valorise mixed organic waste where diverse microorganisms exist. Caproate is the most dominant product in the microbial chain elongation of mixed organic waste and ethanol, which can be produced at a high rate and selectivity. Caproate has a higher economic value, a lower solubility in water and an interesting market potential. Thus, caproic acid production from mixed organic waste and ethanol via microbial chain elongation is currently undergoing up-scaling and commercialisation.

Many studies were done to improve the process of caproate production via microbial chain elongation to make it of industrial interest. The on-going commercialisation of microbial chain elongation also supports the economic feasibility. However, until now, no study addressed the environmental sustainability of microbial chain elongation. Chapter 2 of this thesis took the first attempt in analysing the life-cycle environmental impacts of caproic acid production from organic waste via microbial chain elongation, based on the literature and existing business case. The use of ethanol as a co-substrate (i.e. the electron donor) was shown to be the largest cause the environmental impact. This was found in in all assessed cases and all impact categories studied, and regardless of the feedstocks from which ethanol was produced. An alternative for ethanol as electron donor in microbial chain elongation is, therefore, an effective way to improve the environmental sustainability of microbial chain elongation.

In Chapter 3, we investigated the use of methanol as an alternative electron donor in microbial chain elongation, i.e. methanol chain elongation, for butyrate and caproate production. Methanol chain elongation was previously demonstrated using a pure culture, but never with a mixed culture. To employ organic waste as feedstock, the feasibility of applying 
methanol chain elongation in an open mixed culture condition needs to be investigated. In Chapter 3, it was demonstrated in a batch incubation that methanol chain elongation could occur with a mixed culture, where butyrate was the dominant product $(4.2 \mathrm{~g} / \mathrm{L})$. Caproate production via methanol chain elongation was also demonstrated, though only in a low concentration $(0.1 \mathrm{~g} / \mathrm{L})$. In a continuous reactor operation, continuous butyrate production (1.5 g/L.day) was achieved via microbial chain elongation of acetate and methanol. However, caproate was not observed in the continuous methanol chain elongation. Interestingly, microorganisms that can perform methanol chain elongation were likely present in the inoculum taken from a previous ethanol chain elongation reactor without any methanol supplement.

In Chapter 4, the use of methanol chain elongation to synthesise a novel product, i.e. isobutyrate, was proposed and investigated. Methanol chain elongation was found to continuously produce butyrate as the main metabolite, the accumulation of which was found to trigger isobutyrate formation in several previous methanogenic anaerobic digestion studies. It was, therefore, hypothesised that by elevating the butyrate concentration in the medium, methanol chain elongation might be able to produce isobutyrate as another metabolite. The result showed that isobutyrate could be produced as the main product, up to $6.2 \mathrm{~g} / \mathrm{L}$, when using acidified supermarket food waste and methanol as the substrate. A continuous methanol chain elongation using synthetic medium was also performed, which achieved a production rate of $2.0 \mathrm{~g} / \mathrm{L}$.day over five hydraulic retention times. Moreover, the production of isovalerate was also observed. Isobutyrate has a much larger market potential than caproate, though its production relies wholly on fossil-based feedstock. Isobutyrate biosynthesis was demonstrated in previous studies, but was only achieved using metabolically engineered microorganisms as the biocatalyst and glucose as the substrate. Methanol chain elongation, in contrast, could employ derivatives from organic waste as the substrates and a self- 
regenerating mixed culture biocatalyst for producing isobutyrate. Moreover, methanol chain elongation may be integrated into the current microbial chain elongation production facility without a significant infrastructure retrofit. All these advantages make methanol chain elongation an interesting and promising isobutyrate production process. The relatively large market potential of isobutyrate promotes the application of chain elongation and the use of organic waste for value-added chemical production.

In Chapter 5, isobutyrate production was integrated with the caproate production via microbial chain elongation, by concurrently feeding both methanol and ethanol to a mixed culture. The result from Chapter 3 supports the possibility of coexistence of ethanol and methanol chain elongation microorganisms in the same microbiome. In Chapter 4, the possible concurrence of methanol and ethanol chain elongation was also observed. Based on these observations, we hypothesised that methanol and ethanol chain elongation could be integrated to simultaneously produce caproate and isobutyrate. The result showed that such integration was possible when a stable $\mathrm{pH}$ was maintained. When $\mathrm{pH}$ was controlled between $6.2-6.5$ and butyrate was supplied in the medium, caproate and isobutyrate could be produced simultaneously. Additionally, increasing the ethanol feeding rate promoted the chain elongation of butyrate to caproate via ethanol chain elongation. The outcome of this chapter demonstrated the possibility of producing two valuable products in a single reactor with a mixed culture which, coupled with further process improvement, may be of industrial interest.

In Chapter 6, we reflected on the caproate production performance of methanol chain elongation, in comparison with other electron donors used in microbial chain elongation, i.e. ethanol and lactate. Furthermore, we also reflected on the isobutyrate production via methanol chain elongation, in comparison with other emerging products in microbial chain elongation. These reflections could serve as a benchmark for methanol chain elongation as a 
waste management strategy. Based on this benchmarking, we proposed that methanol chain elongation is a promising bioprocess for isobutyrate production but not for caproate production. A potential strategy for improving the isobutyrate production via methanol chain elongation was proposed and discussed. The outcomes of this thesis may contribute to future application and assessments of microbial chain elongation in waste management. It may fuel discussion on how to further promote microbial chain elongation for a more sustainable waste management. 
Microbial chain elongation based on methanol 


\section{References}


(1) EIA International Energy Outlook 2016; U.S. Department of Energy: Washington, DC, 2016.

(2) Pachauri, R. K.; Meyer, L.; Plattner, G.-K.; Stocker, T., IPCC, 2014: Climate Change 2014: Synthesis Report. Contribution of Working Groups I, II and III to the Fifth Assessment Report of the Intergovernmental Panel on Climate Change. IPCC: 2015.

(3) Shafiee, S.; Topal, E., When will fossil fuel reserves be diminished? Energy Policy 2009, 37, (1), 181-189.

(4) Höök, M.; Tang, X., Depletion of fossil fuels and anthropogenic climate change-A review. Energy Policy 2013, 52, 797-809.

(5) Hoornweg, D.; Bhada-Tata, P. What a waste: a global review of solid waste management; World Bank: Washington, DC., 2012; pp 1-98.

(6) Balat, M.; Balat, H., Recent trends in global production and utilization of bio-ethanol fuel. Appl. Energ. 2009, 86, (11), 2273-2282.

(7) Johnston, M.; Holloway, T., A Global Comparison of National Biodiesel Production Potentials. Environ. Sci. Technol. 2007, 41, (23), 7967-7973.

(8) Gerbens-Leenes, P. W.; Hoekstra, A. Y.; van der Meer, T., The water footprint of energy from biomass: A quantitative assessment and consequences of an increasing share of bioenergy in energy supply. Ecol. Econ. 2009, 68, (4), 1052-1060.

(9) Searchinger, T.; Heimlich, R.; Houghton, R. A.; Dong, F.; Elobeid, A.; Fabiosa, J.; Tokgoz, S.; Hayes, D.; Yu, T.-H., Use of U.S. croplands for biofuels increases greenhouse gases through emissions from land-use change. Science. 2008, 319, (5867), 1238-1240.

(10) Yang, Y.; Bae, J.; Kim, J.; Suh, S., Replacing Gasoline with Corn Ethanol Results in Significant Environmental Problem-Shifting. Environ. Sci. Technol. 2012, 46, (7), 36713678.

(11) Rathmann, R.; Szklo, A.; Schaeffer, R., Land use competition for production of food and liquid biofuels: An analysis of the arguments in the current debate. Renew. Energy 2010, $35,(1), 14-22$.

(12) Graham-Rowe, D., Agriculture: Beyond food versus fuel. Nature 2011, 474, (7352), S6S8.

(13) Tilman, D.; Socolow, R.; Foley, J. A.; Hill, J.; Larson, E.; Lynd, L.; Pacala, S.; Reilly, J.; Searchinger, T.; Somerville, C.; Williams, R., Beneficial biofuels- The food, energy, and environment trilemma. Science. 2009, 325, (5938), 270-271.

(14) Lin, C. S. K.; Pfaltzgraff, L. A.; Herrero-Davila, L.; Mubofu, E. B.; Abderrahim, S.; Clark, J. H.; Koutinas, A. A.; Kopsahelis, N.; Stamatelatou, K.; Dickson, F.; Thankappan, S.; Mohamed, Z.; Brocklesby, R.; Luque, R., Food waste as a valuable resource for the production of chemicals, materials and fuels. Current situation and global perspective. Energy Environ. Sci. 2013, 6, 424-464.

(15) Sjöström, E., Wood Chemistry: Fundamentals and Applications. Gulf Professional Publishing: Houston, TX, USA, 1993.

(16) Mondal, P.; Dang, G. S.; Garg, M. O., Syngas production through gasification and cleanup for downstream applications - Recent developments. Fuel Process. Technol. 2011, 92, (8), 1395-1410.

(17) Wilhelm, D. J.; Simbeck, D. R.; Karp, A. D.; Dickenson, R. L., Syngas production for gas-to-liquids applications: technologies, issues and outlook. Fuel Process. Technol. 2001, $71,(1-3), 139-148$.

(18) Olah, G. A., Towards Oil Independence Through Renewable Methanol Chemistry. Angew. Chem. Int. Ed. 2013, 52, (1), 104-107.

(19) Richter, H.; Martin, M.; Angenent, L., A Two-Stage Continuous Fermentation System for Conversion of Syngas into Ethanol. Energies 2013, 6, (8), 3987. 
(20) Kleerebezem, R.; van Loosdrecht, M. C. M., Mixed culture biotechnology for bioenergy production. Curr. Opin. Biotechnol. 2007, 18, (3), 207-212.

(21) Renkow, M.; Rubin, A. R., Does municipal solid waste composting make economic sense? J. Environ. Manage. 1998, 53, (4), 339-347.

(22) Steinbusch, K. J. Liquid biofuel production from volatile fatty acids. Ph.D. Dissertation, Wageningen University, Wageningen, 2010.

(23) Angenent, L. T.; Karim, K.; Al-Dahhan, M. H.; Wrenn, B. A.; Domíguez-Espinosa, R., Production of bioenergy and biochemicals from industrial and agricultural wastewater.

Trends Biotechnol. 2004, 22, (9), 477-485.

(24) Chester, M.; Martin, E., Cellulosic Ethanol from Municipal Solid Waste: A Case Study of the Economic, Energy, and Greenhouse Gas Impacts in California. Environ. Sci. Technol. 2009, 43, (14), 5183-5189.

(25) Li, A.; Antizar-Ladislao, B.; Khraisheh, M., Bioconversion of municipal solid waste to glucose for bio-ethanol production. Bioprocess Biosystems Eng. 2007, 30, (3), 189-196.

(26) Agler, M. T.; Wrenn, B. A.; Zinder, S. H.; Angenent, L. T., Waste to bioproduct conversion with undefined mixed cultures: the carboxylate platform. Trends Biotechnol. 2011, 29, (2), 70-78.

(27) Chang, H.; Kim, N.-J.; Kang, J.; Jeong, C., Biomass-derived volatile fatty acid platform for fuels and chemicals. Biotechnol. Bioprocess Eng. 2010, 15, (1), 1-10.

(28) Byrom, D., Polymer synthesis by microorganisms: technology and economics. Trends Biotechnol. 1987, 5, (9), 246-250.

(29) Akiyama, M.; Tsuge, T.; Doi, Y., Environmental life cycle comparison of polyhydroxyalkanoates produced from renewable carbon resources by bacterial fermentation. Polym. Degradation Stab. 2003, 80, (1), 183-194.

(30) Grootscholten, T. I. M. Development of a mixed culture chain elongation process based on municipal solid waste and ethanol. Ph.D. Dissertation, Wageningen University, Wageningen, 2013.

(31) Ding, H.-B.; Tan, G.-Y. A.; Wang, J.-Y., Caproate formation in mixed-culture fermentative hydrogen production. Bioresour. Technol. 2010, 101, (24), 9550-9559.

(32) Steinbusch, K. J. J.; Hamelers, H. V. M.; Plugge, C. M.; Buisman, C. J. N., Biological formation of caproate and caprylate from acetate: Fuel and chemical production from low grade biomass. Energy Environ. Sci. 2011, 4, (1), 216-224.

(33) Angenent, L. T.; Richter, H.; Buckel, W.; Spirito, C. M.; Steinbusch, K. J. J.; Plugge, C. M.; Strik, D. P. B. T. B.; Grootscholten, T. I. M.; Buisman, C. J. N.; Hamelers, H. V. M., Chain elongation with reactor microbiomes: Open-culture biotechnology to produce biochemicals. Environ. Sci. Technol. 2016, 50, (6), 2796-2810.

(34) Spirito, C. M.; Richter, H.; Rabaey, K.; Stams, A. J. M.; Angenent, L. T., Chain elongation in anaerobic reactor microbiomes to recover resources from waste. Curr. Opin. Biotechnol. 2014, 27, (0), 115-122.

(35) Grootscholten, T. I. M.; Steinbusch, K. J. J.; Hamelers, H. V. M.; Buisman, C. J. N., Improving medium chain fatty acid productivity using chain elongation by reducing the hydraulic retention time in an upflow anaerobic filter. Bioresour. Technol. 2013, 136, 735 738.

(36) Grootscholten, T. I. M.; Steinbusch, K. J. J.; Hamelers, H. V. M.; Buisman, C. J. N., Chain elongation of acetate and ethanol in an upflow anaerobic filter for high rate MCFA production. Bioresour. Technol. 2013, 135, (0), 440-445.

(37) Agler, M. T.; Spirito, C. M.; Usack, J. G.; Werner, J. J.; Angenent, L. T., Chain elongation with reactor microbiomes: Upgrading dilute ethanol to medium-chain carboxylates. Energy Environ. Sci. 2012, 5, (8), 8189-8192. 
(38) Grootscholten, T. I. M.; Kinsky dal Borgo, F.; Hamelers, H. V. M.; Buisman, C. J. N., Promoting chain elongation in mixed culture acidification reactors by addition of ethanol. Biomass Bioenergy. 2013, 48, 10-16.

(39) Grootscholten, T. I. M.; Strik, D. P. B. T. B.; Steinbusch, K. J. J.; Buisman, C. J. N.; Hamelers, H. V. M., Two-stage medium chain fatty acid (MCFA) production from municipal solid waste and ethanol. Appl. Energ. 2014, 116, 223-229.

(40) Vasudevan, D.; Richter, H.; Angenent, L. T., Upgrading dilute ethanol from syngas fermentation to n-caproate with reactor microbiomes. Bioresour. Technol. 2014, 151, 378382 .

(41) Seedorf, H.; Fricke, W. F.; Veith, B.; Brüggemann, H.; Liesegang, H.; Strittmatter, A.; Miethke, M.; Buckel, W.; Hinderberger, J.; Li, F.; Hagemeier, C.; Thauer, R. K.; Gottschalk, G., The genome of Clostridium kluyveri, a strict anaerobe with unique metabolic features. Proc. Natl. Acad. Sci. USA. 2008, 105, (6), 2128-2133.

(42) Van Eerten-Jansen, M. C. A. A.; Ter Heijne, A.; Grootscholten, T. I. M.; Steinbusch, K. J. J.; Sleutels, T. H. J. A.; Hamelers, H. V. M.; Buisman, C. J. N., Bioelectrochemical production of caproate and caprylate from acetate by mixed Cultures. ACS Sustainable Chem. Eng. 2013, 1, (5), 513-518.

(43) Kucek, L. A.; Nguyen, M.; Angenent, L. T., Conversion of 1-lactate into n-caproate by a continuously fed reactor microbiome. Water Res. 2016, 93, 163-171.

(44) Zhu, X.; Tao, Y.; Liang, C.; Li, X.; Wei, N.; Zhang, W.; Zhou, Y.; Yang, Y.; Bo, T., The synthesis of n-caproate from lactate: a new efficient process for medium-chain carboxylates production. Sci. Rep. 2015, 5, 14360.

(45) Diender, M.; Stams, A. J. M.; Sousa, D. Z., Production of medium-chain fatty acids and higher alcohols by a synthetic co-culture grown on carbon monoxide or syngas. Biotechnol. Biofuels. 2016, 9, (1), 82.

(46) Ganigué, R.; Sánchez-Paredes, P.; Bañeras, L.; Colprim, J., Low fermentation pH is a trigger to alcohol production, but a killer to chain elongation. Front Microbiol 2016, 7, (702), $1-11$.

(47) Lindley, N. D.; Loubière, P.; Pacaud, S.; Mariotto, C.; Goma, G., Novel products of the acidogenic fermentation of methanol during growth of Eubacterium limosum in the presence of high concentrations of organic acids. J. Gen. Microbiol. 1987, 133, (12), 3557-3563.

(48) Steinbusch, K. J. J.; Arvaniti, E.; Hamelers, H. V. M.; Buisman, C. J. N., Selective inhibition of methanogenesis to enhance ethanol and n-butyrate production through acetate reduction in mixed culture fermentation. Bioresour. Technol. 2009, 100, (13), 3261-3267.

(49) Demirbas, A., Biomethanol production from organic waste materials. Energ Source Part A. 2008, 30, (6), 565-572.

(50) Balan, V.; Chiaramonti, D.; Kumar, S., Review of US and EU initiatives toward development, demonstration, and commercialization of lignocellulosic biofuels. Biofuels, Bioprod. Biorefin. 2013, 7, (6), 732-759.

(51) Lundgren, J.; Ekbom, T.; Hulteberg, C.; Larsson, M.; Grip, C. E.; Nilsson, L.; Tunå, P., Methanol production from steel-work off-gases and biomass based synthesis gas. Appl. Energ. 2013, 112, 431-439.

(52) Gutiérrez Ortiz, F. J.; Serrera, A.; Galera, S.; Ollero, P., Methanol synthesis from syngas obtained by supercritical water reforming of glycerol. Fuel 2013, 105, 739-751.

(53) Tarud, J.; Phillips, S. In Technoeconomic comparison of biofuels: ethanol, methanol, and gasoline from gasification of woody residues, American Chemical Society - National Meeting, Denver, US, 2011; Denver, US, 2011.

(54) Hasegawa, F.; Yokoyama, S.; Imou, K., Methanol or ethanol produced from woody biomass: Which is more advantageous? Bioresour. Technol. 2010, 101, (1), 109-111. 
(55) Genthner, B. R.; Davis, C. L.; Bryant, M. P., Features of rumen and sewage sludge strains of Eubacterium limosum, a methanol- and $\mathrm{H}_{2}-\mathrm{CO}_{2}$-utilizing species. Appl. Environ. Microbiol. 1981, 42, (1), 12-19.

(56) Naik, S. N.; Goud, V. V.; Rout, P. K.; Dalai, A. K., Production of first and second generation biofuels: A comprehensive review. Renew. Sust. Energy. Rev. 2010, 14, (2), 578597.

(57) Zhang, K.; Woodruff, A. P.; Xiong, M.; Zhou, J.; Dhande, Y. K., A synthetic metabolic pathway for production of the platform chemical isobutyric acid. ChemSusChem. 2011, 4, (8), 1068-1070.

(58) Haveren, J. v.; Scott, E. L.; Sanders, J., Bulk chemicals from biomass. Biofuels, Bioprod. Biorefin. 2008, 2, (1), 41-57.

(59) Andersen, S. Electrochemical extraction, recovery and valorization of carboxylic acids from biorefineries. Ph.D. Dissertation, Ghent University, Ghent, 2016.

(60) Hetherington, A. C.; Borrion, A. L.; Griffiths, O. G.; McManus, M. C., Use of LCA as a development tool within early research: challenges and issues across different sectors. Int. $J$. Life Cycle Assessment. 2013, 19, (1), 130-143.

(61) Cherubini, F., The biorefinery concept: Using biomass instead of oil for producing energy and chemicals. Energy Convers. Manage. 2010, 51, (7), 1412-1421.

(62) Tuck, C. O.; Pérez, E.; Horváth, I. T.; Sheldon, R. A.; Poliakoff, M., Valorization of biomass: Deriving more value from waste. Science. 2012, 337, (6095), 695-699.

(63) Sanders, J.; Scott, E.; Weusthuis, R.; Mooibroek, H., Bio-refinery as the bio-inspired process to bulk chemicals. Macromol. Biosci. 2007, 7, (2), 105-117.

(64) Kleerebezem, R.; Joosse, B.; Rozendal, R.; Van Loosdrecht, M. C. M., Anaerobic digestion without biogas? Rev. Environ. Sci. Technol. 2015, 14, (4), 787-801.

(65) Levy, P. F.; Sanderson, J. E.; Kispert, R. G.; Wise, D. L., Biorefining of biomass to liquid fuels and organic chemicals. Enzyme Microb. Technol. 1981, 3, (3), 207-215.

(66) Ge, S.; Usack, J. G.; Spirito, C. M.; Angenent, L. T., Long-term n-caproic acid production from yeast-fermentation beer in an anaerobic bioreactor with continuous product extraction. Environ. Sci. Technol. 2015, 49, (13), 8012-8021.

(67) Zentek, J.; Buchheit-Renko, S.; Ferrara, F.; Vahjen, W.; Van Kessel, A. G.; Pieper, R., Nutritional and physiological role of medium-chain triglycerides and medium-chain fatty acids in piglets. Anim. Health Res. Rev. 2011, 12, (1), 83-93.

(68) Woolford, M. K., Microbiological screening of the straight chain fatty acids (C1-C12) as potential silage additives. J. Sci. Food Agric. 1975, 26, (2), 219-228.

(69) Scalschi, L.; Vicedo, B.; Camañes, G.; Fernandez-Crespo, E.; Lapeña, L.; GonzálezBosch, C.; García-Agustín, P., Hexanoic acid is a resistance inducer that protects tomato plants against Pseudomonas syringae by priming the jasmonic acid and salicylic acid pathways. Mol. Plant Pathol. 2013, 14, (4), 342-355.

(70) Coma, M.; Vilchez-Vargas, R.; Roume, H.; Jauregui, R.; Pieper, D. H.; Rabaey, K., Product diversity linked to substrate usage in chain elongation by mixed-culture fermentation. Environ. Sci. Technol. 2016, 50, (12), 6467-6476.

(71) Grootscholten, T. I. M.; Steinbusch, K. J. J.; Hamelers, H. V. M.; Buisman, C. J. N., High rate heptanoate production from propionate and ethanol using chain elongation.

Bioresour. Technol. 2013, 136, 715-718.

(72) Liang, S.; Wan, C., Carboxylic acid production from brewer's spent grain via mixed culture fermentation. Bioresour. Technol. 2015, 182, 179-183.

(73) Roghair, M.; Strik, D. P. B. T. B.; Steinbusch, K. J. J.; Weusthuis, R. A.; Bruins, M. E.; Buisman, C. J. N., Granular sludge formation and characterization in a chain elongation process. Process Biochem. 2016, 51, (10), 1594-1598. 
(74) Weimer, P. J.; Nerdahl, M.; Brandl, D. J., Production of medium-chain volatile fatty acids by mixed ruminal microorganisms is enhanced by ethanol in co-culture with Clostridium kluyveri. Bioresour. Technol. 2015, 175, 97-101.

(75) Xu, J.; Guzman, J. J. L.; Andersen, S. J.; Rabaey, K.; Angenent, L. T., In-line and selective phase separation of medium-chain carboxylic acids using membrane electrolysis. Chem. Commun. 2015, 51, (31), 6847-6850.

(76) Xiong, B.; Richard, T. L.; Kumar, M., Integrated acidogenic digestion and carboxylic acid separation by nanofiltration membranes for the lignocellulosic carboxylate platform. $J$. Membr. Sci. 2015, 489, 275-283.

(77) Kannengiesser, J.; Sakaguchi-Söder, K.; Mrukwia, T.; Jager, J.; Schebek, L., Extraction of medium chain fatty acids from organic municipal waste and subsequent production of biobased fuels. Waste Manage. 2016, 47, Part A, 78-83.

(78) IES-JRC-EU, International Reference Life Cycle Data System (ILCD) Handbook General Guide for Life Cycle Assessment - Detailed Guidance. 1st ed.; Publications Office of the European Union: Luxemburg, 2010.

(79) Ahlgren, S.; Björklund, A.; Ekman, A.; Karlsson, H.; Berlin, J.; Börjesson, P.; Ekvall, T.; Finnveden, G.; Janssen, M.; Strid, I., Review of methodological choices in LCA of biorefinery systems - key issues and recommendations. Biofuels, Bioprod. Biorefin. 2015, 9 , (5), 606-619.

(80) Tufvesson, P.; Ekman, A.; Sardari, R. R. R.; Engdahl, K.; Tufvesson, L., Economic and environmental assessment of propionic acid production by fermentation using different renewable raw materials. Bioresour. Technol. 2013, 149, (0), 556-564.

(81) Weidema, B. P. B., Ch.; Hischier, R.; Mutel, Ch.; Nemecek, T.; Reinhard, J.; Vadenbo, C.O.; Wernet, G, The ecoinvent database: Overview and methodology, Data quality guideline for the ecoinvent database version 3. In Ecoinvent: Zurich, Switzerland, 2013.

(82) Tufvesson, L. M.; Tufvesson, P.; Woodley, J. M.; Börjesson, P., Life cycle assessment in green chemistry: overview of key parameters and methodological concerns. Int. J. Life Cycle Assessment. 2013, 18, (2), 431-444.

(83) Jungbluth, N.; Chudacoff, M.; Dauriat, A.; Dinkel, F.; Doka, G.; Faist Emmenegger, M.; Gnansounou, E.; Kljun, N.; Schleiss, K.; Spielmann, M. Life cycle inventories of bioenergy; Swiss Centre for Life Cycle Inventories Dübendorf, 2007.

(84) Kim, S.; Dale, B. E., Life cycle assessment of fuel ethanol derived from corn grain via dry milling. Bioresour. Technol. 2008, 99, (12), 5250-5260.

(85) Arslan, D. Selective short chain carboxylates production by mixed culture fermentation. Ph.D. Dissertation, Wageningen University, Wageningen, 2014.

(86) Ren, N.; Wang, B.; Huang, J.-C., Ethanol-type fermentation from carbohydrate in high rate acidogenic reactor. Biotechnol. Bioeng. 1997, 54, (5), 428-433.

(87) Arslan, D.; Steinbusch, K. J. J.; Diels, L.; De Wever, H.; Buisman, C. J. N.; Hamelers, H. V. M., Effect of hydrogen and carbon dioxide on carboxylic acids patterns in mixed culture fermentation. Bioresour. Technol. 2012, 118, (0), 227-234.

(88) Steinbusch, K. J. J.; Hamelers, H. V. M.; Buisman, C. J. N., Alcohol production through volatile fatty acids reduction with hydrogen as electron donor by mixed cultures. Water Res. 2008, 42, (15), 4059-4066.

(89) Arslan, D.; Steinbusch, K. J. J.; Diels, L.; Hamelers, H. V. M.; Strik, D. P. B. T. B.; Buisman, C. J. N.; De Wever, H., Selective short-chain carboxylates production: A review of control mechanisms to direct mixed culture fermentations. Crit. Rev. Environ. Sci. Technol. 2016, 46, (6), 592-634.

(90) Chen, W. S.; Ye, Y.; Steinbusch, K. J. J.; Strik, D. P. B. T. B.; Buisman, C. J. N., Methanol as an alternative electron donor in chain elongation for butyrate and caproate formation. Biomass Bioenergy. 2016, 93, 201-208. 
(91) Chen, W.-S.; Huang, S.; Strik, D. P. B. T. B.; Buisman, C. J. N., Isobutyrate biosynthesis via methanol chain elongation: Converting organic wastes to platform chemicals. J. Chem. Technol. Biotechnol. 2016, in press.

(92) Andersen, S. J.; Candry, P.; Basadre, T.; Khor, W. C.; Roume, H.; Hernandez-Sanabria, E.; Coma, M.; Rabaey, K., Electrolytic extraction drives volatile fatty acid chain elongation through lactic acid and replaces chemical $\mathrm{pH}$ control in thin stillage fermentation. Biotechnol. Biofuels. 2015, 8, (1), 1-14.

(93) Foley, J. M.; Rozendal, R. A.; Hertle, C. K.; Lant, P. A.; Rabaey, K., Life cycle assessment of high-rate anaerobic treatment, microbial fuel cells, and microbial electrolysis cells. Environ. Sci. Technol. 2010, 44, (9), 3629-3637.

(94) Jourdin, L.; Freguia, S.; Flexer, V.; Keller, J., Bringing high-Rate, $\mathrm{CO}_{2}$-based microbial electrosynthesis closer to practical implementation through improved electrode design and operating conditions. Environ. Sci. Technol. 2016, 50, (4), 1982-1989.

(95) Carballa, M.; Duran, C.; Hospido, A., Should we pretreat solid waste prior to anaerobic digestion? an assessment of its environmental cost. Environ. Sci. Technol. 2011, 45, (24), 10306-10314.

(96) de Vries, S. C.; van de Ven, G. W. J.; van Ittersum, M. K.; Giller, K. E., Resource use efficiency and environmental performance of nine major biofuel crops, processed by firstgeneration conversion techniques. Biomass Bioenergy. 2010, 34, (5), 588-601.

(97) Muñoz, I.; Flury, K.; Jungbluth, N.; Rigarlsford, G.; Canals, L. M.; King, H., Life cycle assessment of bio-based ethanol produced from different agricultural feedstocks. Int. J. Life Cycle Assessment. 2013, 19, (1), 109-119.

(98) von Blottnitz, H.; Curran, M. A., A review of assessments conducted on bio-ethanol as a transportation fuel from a net energy, greenhouse gas, and environmental life cycle perspective. J. Clean. Prod. 2007, 15, (7), 607-619.

(99) Chum, H. L.; Warner, E.; Seabra, J. E. A.; Macedo, I. C., A comparison of commercial ethanol production systems from Brazilian sugarcane and US corn. Biofuels, Bioprod. Biorefin. 2014, 8, (2), 205-223.

(100) Muñoz, I.; Rigarlsford, G.; i Canals, L. M.; King, H., Accounting for greenhouse gas emissions from the degradation of chemicals in the environment. Int. J. Life Cycle Assessment. 2012, 18, (1), 252-262.

(101) Schmitt, E.; Bura, R.; Gustafson, R.; Cooper, J.; Vajzovic, A., Converting lignocellulosic solid waste into ethanol for the State of Washington: An investigation of treatment technologies and environmental impacts. Bioresour. Technol. 2012, 104, 400-409. (102) Thannimalay, L.; Yusoff, S.; Zawawi, N. Z., Life cycle assessment of sodium hydroxide. Aust. J. Basic Appl. Sci. 2013, 7, (2), 421-431.

(103) Gurieff, N.; Lant, P., Comparative life cycle assessment and financial analysis of mixed culture polyhydroxyalkanoate production. Bioresour. Technol. 2007, 98, (17), 33933403.

(104) Heimersson, S.; Morgan-Sagastume, F.; Peters, G. M.; Werker, A.; Svanström, M., Methodological issues in life cycle assessment of mixed-culture polyhydroxyalkanoate production utilising waste as feedstock. N. Biotechnol. 2014, 31, (4), 383-393.

(105) Evangelisti, S.; Lettieri, P.; Borello, D.; Clift, R., Life cycle assessment of energy from waste via anaerobic digestion: A UK case study. Waste Manage. 2014, 34, (1), 226-237.

(106) Renz, M., Ketonization of carboxylic acids by decarboxylation: Mechanism and scope. Eur. J. Org. Chem. 2005, 2005, (6), 979-988.

(107) Bagby, M. O.; Johnson, R. W.; Daniels, R. W.; Contrell, R. R.; Sauer, E. T.; Keenan, M. J.; Krevalis, M. A., Carboxylic Acids. In Kirk-Othmer Encyclopedia of Chemical Technology, John Wiley \& Sons, Inc.: Hoboken, 2000. 
(108) Landis, D. A.; Gardiner, M. M.; van der Werf, W.; Swinton, S. M., Increasing corn for biofuel production reduces biocontrol services in agricultural landscapes. Proc. Natl. Acad. Sci. USA. 2008, 105, (51), 20552-20557.

(109) Kim, S.; Dale, B. E., Global potential bioethanol production from wasted crops and crop residues. Biomass Bioenergy. 2004, 26, (4), 361-375.

(110) Tarasov, A. L.; Borzenkov, I. A.; Belyayev, S. S., Investigation of the trophic relations between anaerobic microorganisms from an underground gas repository during methanol utilization. Microbiology 2011, 80, (2), 180-187.

(111) Barker, H. A.; Kamen, M. D.; Bornstein, B. T., The synthesis of butyric and caproic acids from ethanol and acetic acid by Clostridium Kluyveri. Proc. Natl. Acad. Sci. USA. 1945, 31, (12), 9 .

(112) Pacaud, S.; Loubiere, P.; Goma, G., Methanol metabolism by Eubacterium limosum $\mathrm{B} 2$ : Effects of $\mathrm{pH}$ and carbon dioxide on growth and organic acid production. Curr. Microbiol. 1985, 12, (5), 245-250.

(113) Phillips, J. R.; Klasson, K. T.; Clausen, E. C.; Gaddy, J. L., Biological production of ethanol from coal synthesis gas. Appl. Biochem. Biotechnol. 1993, 39-40, (1), 559-571.

(114) Duboc, P.; Schill, N.; Menoud, L.; Van Gulik, W.; Von Stockar, U., Measurements of sulfur, phosphorus and other ions in microbial biomass: influence on correct determination of elemental composition and degree of reduction. J. Biotechnol. 1995, 43, (2), 145-158.

(115) Ljungdhal, L., The autotrophic pathway of acetate synthesis in acetogenic bacteria. Annu. Rev. Microbiol. 1986, 40, (1), 415-450.

(116) Loubière, P.; Lindley, N. D., The use of acetate as an additional co-substrate improves methylotrophi growth of the acetogenic anaerobe Eubacterium limosum when $\mathrm{CO}_{2}$ fixation is rate-limiting. J. Gen. Microbiol. 1991, 137, (9), 2247-2251.

(117) Pacaud, S.; Loubière, P.; Goma, G.; Lindley, N. D., Organic acid production during methylotrophic growth of Eubacterium limosum B2: displacement towards increased butyric acid yields by supplementing with acetate. Appl. Microbiol. Biotechnol. 1986, 23, (5), 330335 .

(118) Demirbas, A., Biofuels sources, biofuel policy, biofuel economy and global biofuel projections. Energy Convers. Manage. 2008, 49, (8), 2106-2116.

(119) Sheldon, R. A., Green and sustainable manufacture of chemicals from biomass: state of the art. Green Chem. 2014, 16, (3), 950-963.

(120) Ai, M.; Muneyama, E.; Kunishige, A.; Ohdan, K., Formation of methacrylic acid by oxidative dehydrogenation of isobutyric acid: Promoters of iron phosphate catalysts. Appl. Catal., A. 1994, 109, (1), 135-146.

(121) Dodds, D. R.; Gross, R. A., Chemicals from biomass. Science. 2007, 318, (5854), $1250-1251$.

(122) Lang, K.; Zierow, J.; Buehler, K.; Schmid, A., Metabolic engineering of Pseudomonas sp. strain VLB120 as platform biocatalyst for the production of isobutyric acid and other secondary metabolites. Microb. Cell Fact. 2014, 13, (1), 2.

(123) Lang, K.; Buehler, K.; Schmid, A., Multistep synthesis of (s)-3-hydroxyisobutyric acid from glucose using Pseudomonas taiwanensis VLB120 B83 T7 catalytic biofilms. Adv. Synth. Catal. 2015, 357, (8), 1919-1927.

(124) Perez, J. M.; Richter, H.; Loftus, S. E.; Angenent, L. T., Biocatalytic reduction of short-chain carboxylic acids into their corresponding alcohols with syngas fermentation. Biotechnol. Bioeng. 2013, 110, (4), 1066-1077.

(125) Karabektas, M.; Hosoz, M., Performance and emission characteristics of a diesel engine using isobutanol-diesel fuel blends. Renew. Energy 2009, 34, (6), 1554-1559. (126) Atsumi, S.; Hanai, T.; Liao, J. C., Non-fermentative pathways for synthesis of branched-chain higher alcohols as biofuels. Nature 2008, 451, (7174), 86-89. 
(127) Tao, L.; Tan, E. C. D.; McCormick, R.; Zhang, M.; Aden, A.; He, X.; Zigler, B. T., Techno-economic analysis and life-cycle assessment of cellulosic isobutanol and comparison with cellulosic ethanol and n-butanol. Biofuels, Bioprod. Biorefin. 2014, 8, (1), 30-48. (128) Lovley, D. R.; Klug, M. J., Intermediary metabolism of organic matter in the sediments of a eutrophic lake. Appl. Environ. Microbiol. 1982, 43, (3), 552-560.

(129) Wang, Q.; Kuninobu, M.; Ogawa, H. I.; Kato, Y., Degradation of volatile fatty acids in highly efficient anaerobic digestion. Biomass Bioenergy. 1999, 16, (6), 407-416.

(130) Lata, K.; Rajeshwari, K. V.; Pant, D. C.; Kishore, V. V. N., Volatile fatty acid production during anaerobic mesophilic digestion of tea and vegetable market wastes. World J. Microbiol. Biotechnol. 2002, 18, (6), 589-592.

(131) Angelidaki, I.; Ahring, B. K., Isomerization of n- and i-butyrate in anaerobic methanogenic systems. Antonie Van Leeuwenhoek. 1995, 68, (4), 285-291.

(132) Mechichi, T.; Sayadi, S., Evaluating process imbalance of anaerobic digestion of olive mill wastewaters. Process Biochem. 2005, 40, (1), 139-145.

(133) Matthies, C.; Schink, B., Reciprocal isomerization of butyrate and isobutyrate by the strictly anaerobic bacterium strain WoG13 and methanogenic isobutyrate degradation by a defined triculture. Appl. Environ. Microbiol. 1992, 58, (5), 1435-1439.

(134) Tholozan, J.-L.; Samain, E.; Grivet, J.-P., Isomerization between n-butyrate and isobutyrate in enrichment cultures. FEMS Microbiol. Lett. 1988, 53, (3), 187-191.

(135) Zinder, S. H.; Cardwell, S. C.; Anguish, T.; Lee, M.; Koch, M., Methanogenesis in a thermophilic $\left(58^{\circ} \mathrm{C}\right)$ anaerobic digestor: Methanothrix sp. as an important aceticlastic methanogen. Appl. Environ. Microbiol. 1984, 47, (4), 796-807.

(136) Hajarnis, S. R.; Ranade, D. R., Inhibition of methanogens by n- and iso-volatile fatty acids. World J. Microbiol. Biotechnol. 1994, 10, (3), 350-351.

(137) Yuan, H.; Zhu, N., Progress in inhibition mechanisms and process control of intermediates and by-products in sewage sludge anaerobic digestion. Renew. Sust. Energy. Rev. 2016, 58, 429-438.

(138) van der Meijden, P.; van der Drift, C.; Vogels, G., Methanol conversion in Eubacterium limosum. Arch. Microbiol. 1984, 138, (4), 360-364.

(139) Stieb, M.; Schink, B., Anaerobic degradation of isobutyrate by methanogenic enrichment cultures and by a Desulfococcus multivorans strain. Arch. Microbiol. 1989, 151, (2), 126-132.

(140) Heijthuijsen, J. H. F. G.; Hansen, T. A., Interspecies hydrogen transfer in co-cultures of methanol-utilizing acidogens and sulfate-reducing or methanogenic bacteria. FEMS Microbiol. Lett. 1986, 38, (1), 57-64.

(141) Oude Elferink, S. J. W. H.; Lens, P. N. L.; Dijkema, C.; Stams, A. J. M., Isomerization of butyrate to isobutyrate by Desulforhabdus amnigenus. FEMS Microbiol. Lett. 1996, 142, (2-3), 237-241.

(142) Loubiere, P.; Goma, G.; Lindley, N. D., A non-passive mechanism of butyrate excretion operates during acidogenic fermentation of methanol by Eubacterium limosum. Antonie Van Leeuwenhoek. 1990, 57, (2), 83-89.

(143) Banerjee, R.; Ragsdale, S. W., The many faces of Vitamin B12: Catalysis by cobalamin-dependent enzymes. Annu. Rev. Biochem. 2003, 72, (1), 209-247.

(144) Oh, S.-E.; Van Ginkel, S.; Logan, B. E., The relative effectiveness of ph control and heat treatment for enhancing biohydrogen gas production. Environ. Sci. Technol. 2003, 37, (22), 5186-5190.

(145) Chen, W.-S.; Strik, D. P. B. T. B.; Buisman, C. J. N.; Kroeze, C. In Environmental Impact Assessment of caproic acid production from organic waste: A case study of a novel pilot-scale biorefinery in the Netherlands, EXPO 2015 LCA for "Feeding the planet and 
energy for life", Milan, Italy, 2015; Scalbi, S.; Loprieno, A. D.; Sposato, P., Eds. Milan, Italy, 2015.

(146) Kenealy, W. R.; Waselefsky, D. M., Studies on the substrate range of Clostridium kluyveri; the use of propanol and succinate. Arch. Microbiol. 1985, 141, (3), 187-194.

(147) Smith, M. R.; Mah, R. A., Growth and methanogenesis by Methanosarcina strain 227 on acetate and methanol. Appl. Environ. Microbiol. 1978, 36, (6), 870-879.

(148) Pacaud, S.; Loubiere, P.; Goma, G.; Lindley, N. D., Effects of various organic acid supplements on growth rates of Eubacterium limosum B2 on methanol. Appl. Microbiol. Biotechnol. 1986, 24, (1), 75-78.

(149) Loubiere, P.; Lindley, N. D., Methylotrophic growth of a mutant strain of the acetogenic bacterium Eubacterium limosum that uses acetate as co-substrate in the absence of $\mathrm{CO}_{2}$. FEMS Microbiol. Lett. 1994, 123, (3), 281-287.

(150) Tomlinson, N.; Barker, H. A., Carbon dioxide and acetate utilization by Clostridium kluyveri. I. Influence of nutritional conditions on utilization patterns. J. Biol. Chem. 1954, 209, (2), 585-595.

(151) Raes, S. M. T.; Jourdin, L.; Buisman, C. J. N.; Strik, D. P. B. T. B., Continuous longterm bioelectrochemical chain elongation to butyrate. ChemElectroChem 2017, 4, (2), 386395.

(152) Ballesteros, M.; Sáez, F.; Ballesteros, I.; Manzanares, P.; Negro, M. J.; Martínez, J. M.; Castañeda, R.; Oliva Dominguez, J. M., Ethanol production from the organic fraction obtained after thermal pretreatment of municipal solid waste. Appl. Biochem. Biotechnol. 2010, 161, (1), 423-431.

(153) DeMoss, R. D.; Bard, R. C.; Gunsalus, I. C., The mechanism of the heterolactic fermentation: a new route of ethanol formation. J. Bacteriol. 1951, 62, (4), 499-511.

(154) Kucek, L. A.; Spirito, C. M.; Angenent, L. T., High n-caprylate productivities and specificities from dilute ethanol and acetate: chain elongation with microbiomes to upgrade products from syngas fermentation. Energy Environ. Sci. 2016, 9, (11), 3482-3494.

(155) Ekman, A.; Börjesson, P., Environmental assessment of propionic acid produced in an agricultural biomass-based biorefinery system. J. Clean. Prod. 2011, 19, (11), 1257-1265.

(156) Nagai, K., New developments in the production of methyl methacrylate. Appl. Catal., A. 2001, 221, (1-2), 367-377.

(157) Dalal, R. K.; Akedo, M.; Cooney, C. L.; Sinskey, A. J., Microbial route for acrylic acid production. Biosources Dig. 1980, 89-97.

(158) Yalkowsky, S. H.; He, Y.; Jain, P., Handbook of aqueous solubility data. CRC press: 2016.

(159) Kortüm, G.; Vogel, W.; Andrussow, K., Disssociation constants of organic acids in aqueous solution. Pure Appl. Chem. 1960, 1, (2-3), 187-536.

(160) Zigová, J.; Šturdík, E.; Vandák, D.; Schlosser, Š., Butyric acid production by Clostridium butyricum with integrated extraction and pertraction. Process Biochem. 1999, 34, (8), 835-843.

(161) Wu, Z.; Yang, S.-T., Extractive fermentation for butyric acid production from glucose by Clostridium tyrobutyricum. Biotechnol. Bioeng. 2003, 82, (1), 93-102.

(162) Dacosta, C. F.; Posada, J. A.; Ramirez, A., Large scale production of

polyhydroxyalkanoates (PHAs) from wastewater: a study of techno-economics, energy use and greenhouse gas emissions. Int J Environ Chem Ecol Geol Geophys Eng 2015, 9, 422-427.

(163) Leong, Y. K.; Show, P. L.; Lin, H. C.; Chang, C. K.; Loh, H.-S.; Lan, J. C.-W.; Ling,

T. C., Preliminary integrated economic and environmental analysis of polyhydroxyalkanoates (PHAs) biosynthesis. Bioresources Bioproc. 2016, 3, (1), 41.

(164) Fernández-Dacosta, C.; Posada, J. A.; Kleerebezem, R.; Cuellar, M. C.; Ramirez, A., Microbial community-based polyhydroxyalkanoates (PHAs) production from wastewater: 
Techno-economic analysis and ex-ante environmental assessment. Bioresour. Technol. 2015, 185, 368-377.

(165) Al-Salem, S. M.; Lettieri, P.; Baeyens, J., Recycling and recovery routes of plastic solid waste (PSW): A review. Waste Manage. 2009, 29, (10), 2625-2643.

(166) Brachi, P.; Chirone, R.; Miccio, F.; Miccio, M.; Picarelli, A.; Ruoppolo, G., Fluidized bed co-gasification of biomass and polymeric wastes for a flexible end-use of the syngas:

Focus on bio-methanol. Fuel 2014, 128, 88-98. 
Acknowledgement 
My first acknowledgement goes to my beloved parents, who just had a big relief after hearing that my thesis was approved. I know you both, especially Mom, are always so worried about my studies, from my primary school to BSc to MSc and even to $\mathrm{PhD}$. Now you can have a true relief, as I don't see myself pursue another degree in this life. I also want to thank you for supporting me going abroad for my study and career. It has been a nice journey and will continue being a great adventure which I very much look forwards to. I also want to say thank you to my dear brother, Barry, who is one of the closest person in my life. Living in different continents did not distance us from each other. I really appreciate and like our constant contact and mutual visits, which have been very comforting and supportive to me. My great acknowledgement also goes to Dandan, my beloved girlfriend. It has been great to have you in my life, which has added so much colors and flavors to my life. Thank you also for taking such good care of me, especially in the last mile of my $\mathrm{PhD}$. I could not imagine completing my $\mathrm{PhD}$ without you being in my journey. I also would like to thank other members in my family, especially Uncle Simon who has been mentoring me during my $\mathrm{PhD}$ journey.

I would like to thank the chair group ETE and all the ETErs. I am very lucky to have such a nice chair group with many inspiring and fun people. To my ETE promoter Cees and supervisor David, I truly acknowledge the space you gave me to explore the research I want to do and the chance you gave me to step into a new, growing science field even with my distinct background. Both of your guidance was extremely valuable to me. Shokouh, Koen, Sanne, Mark, Yvonne, Pim, Arnoud, Indra, Nora, Annemiek, Els and Ilse Voskamp, all of my old ETE mates, I have known all of you since the very beginning of my $\mathrm{PhD}$ or even before. Thank you for all the great time, company, fun and togetherness we had together. Alette, Miriam, Jan Weijma, Kasia, Tim Grotenhuis, Renata, Grietje, Azie, Lucia, Leire, Rosanne, Justine, Ludo, Delaram, Tiemen, Darja and Jan Vreeburg, whom I got to know and interact 
more later in my $\mathrm{PhD}$, I also would like to thank you for the nice cooperation and inspiring talks we had. To Emilius and Laura, my favourite squash mates, I really enjoy the regular squash game and the nice chitchat in between, which has been an important part of my $\mathrm{PhD}$ life. Lots of gratitude also to the supporting team, Hans, Katje, Jean, Ilse, Vinnie, Bert, Liesbeth, Gea and Livio for all the powerful supports during my $\mathrm{PhD}$. My $\mathrm{PhD}$ life would be miserable without all your support. I would also like to thank Huub for several collaborations we had, from improving the communication in ETE, the China $\mathrm{PhD}$ trip and the MetSol programme. I did enjoy working with you very much. Last but not least, I would like to express my appreciation to the fun, open-minded ETE Chinese community including Wenbo, Yujie, Yin, Yingdi, Baocong and Celia. Thank you for all the nice Chinese dinners, weekend outings and the wonderful China $\mathrm{PhD}$ trip we did together.

Several acknowledgements also dedicate to some previous ETErs. David Triana, thank you for being one of my closet friends in Wageningen and a great mentor in my research as well as my life. Special thank also to Kirsten, who brought me into this $\mathrm{PhD}$ position and this interesting field, and helped me a lot during the beginning of my $\mathrm{PhD}$; my gratitude to you is beyond words. Suman, my oldest Wageningen friend, thank you for all the nice time we had together in the last 7 years in Wagenignen. During my PhD, I worked with several inspiring and excellent MSc and BSc students, who also contributed to the accomplishment of this thesis. Tobias, Bart, Yin, Ulysse, Yifan, Shengle and Andre, I would like to acknowledge you for choosing to work with me and the pleasant working time we had together.

My special thanks also go to my other promoter, Carolien Kroeze as well as her famous Miss Nutrients. Carolien, you are a great promoter with a kind, friendly personality, so much patience and very comprehensive advices. I am so glad to have Cees and you as my $\mathrm{PhD}$ promoters. To the Miss Nutrients, Maryna, Mengru and Ang, thank you for all the great coffee breaks we had in ESA and the nice China trip we had together. 
I also want to acknowledge several people and organisations that helped along my $\mathrm{PhD}$ journey. Caroline Plugge, thank you for strengthening my knowledge in microbiology and for all your contribution to my publications. Niels and Maurice from ChainCraft B.V., thank you for supporting my research both scientifically and financially. My gratitude also dedicates to Taiwanese government and Delta electronic foundation, both of which awarded me prestigious prizes for my $\mathrm{PhD}$ research.

I also want to thank my Taiwanese friends, those I met in Wageningen: Hsuan, Eunice, Yuanyuan, Jessie Knag, Tze-yi, I-Chiao, Charlize, Gary Lu, Han Tang, Chia-Chi, Changlin and Pengying. I really like the Taiwanese community we have, and I enjoyed a lot hanging out with your guys. I also want to thank my other group of Taiwanese friends from my BSc: Ganwei, Chun-Kai, 5L, Tobe, Meng-Che, Johnson Tseng, Zhelin Chen, Xiangyu Mo and Johnny Guo; I feel like being home whenever chatting or meeting you guys. Thank you for all the funny, non-sense chats we always have, which I really enjoy and also relieves me from the crazy academia.

Last but not least, after acknowledging the countless people who have shared, accompanied and helped my $\mathrm{PhD}$ journey, I would like to commemorate my beloved granddad, who passed away right before I decided to start my $\mathrm{PhD}$ journey. Dear Grandpa, you did not have much education, but you did teach me how to be a decent and strong person and do the useful things. I have started to acknowledge this more and more, along my $\mathrm{PhD}$ journey. I knew you were always proud of me and my achievement; I want you to know that I am also proud of being your grandson and spending so much of my childhood and teenage with you. I dedicate this dissertation to you, my beloved grandpa. 
Appendix 


\title{
Publication list
}

\author{
Scientific journals
}

Chen, W.S.; Ye, Y.; Steinbusch, K. J. J.; Strik, D. P. B. T. B.; Buisman, C. J. N., Methanol as an alternative electron donor in chain elongation for butyrate and caproate formation. Biomass Bioenergy. 2016, 93, 201-208.

Chen, W.S.; Huang, S.; Strik, D. P. B. T. B.; Buisman, C. J. N., Isobutyrate biosynthesis via methanol chain elongation: Converting organic wastes to platform chemicals. J. Chem. Technol. Biotechnol. 2016, in press.

Chen, W.S.; Strik, D. P. B. T. B.; Buisman, C. J. N.; Kroeze, C., Production of caproic acid from mixed organic waste- an environmental life cycle perspective. Environ. Sci. Technol. Accepted $17^{\text {th }}$ May 2017.

Chen, W.S.; Huang, S.; Strik, D. P. B. T. B.; Buisman, C. J. N., Concurrent use of methanol and ethanol for chain-elongating short chain fatty acids into caproate and isobutyrate., to be submitted.

Helder, M.; Chen, W.S.; van der Harst, E. J. M.; Strik, D. P. B. T. B.; Hamelers, H. V. M.; Buisman, C. J. N.; Potting, J. Electricity production with living plants on a green roof: environmental performance of the plant-microbial fuel cell. Biofuels, Bioprod. Biorefin. 2013, 7 (1), 52-64.

Chen, W.S.; Yang, C.H.; Chang, J.K.; Tsai, W.T. Nieh, J.K.; Wu, Y.P. Hydrogen storage performance of mechanical $\mathrm{Mg} 2 \mathrm{Ni}$ alloy evaluated with a high-pressure microbalance. $J$. Chin. Soc. Mech. Eng. 2013, 34 (2), 97-102.

\section{Conference proceedings}

Chen, W. S.; Roghair, M.; Triana Mecerreyes, D.; Strik, D. P. B. T. B.; Kroeze, C.; Buisman, C. J. N., Mixed Culture Chain Elongation (MCCE) - A Novel Biotechnology for Renewable Biochemical Production from Organic Residual Streams. In Energy Engineering: Proceedings of CAETS 2015 Convocation on Pathways to Sustainability, Raghavan, K. V.; Ghosh, P., Eds. Springer Singapore: Singapore, 2017; pp 157-158.

Chen, W.-S.; Strik, D. P. B. T. B.; Buisman, C. J. N.; Kroeze, C. In Environmental Impact Assessment of caproic acid production from organic waste: A case study of a novel pilotscale biorefinery in the Netherlands, EXPO 2015 LCA for "Feeding the planet and energy for life", Milan, Italy, 2015; Scalbi, S.; Loprieno, A. D.; Sposato, P., Eds. Milan, Italy, 2015. 


\section{About the author}

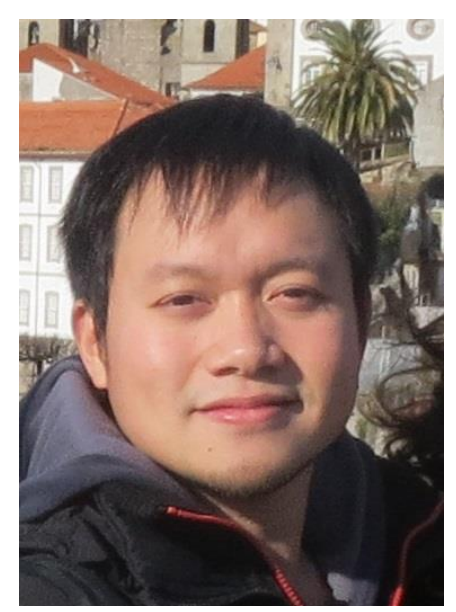

Wei-Shan Chen (momo) was born on $29^{\text {th }}$ June 1987 in Taipei City, Taiwan. He received his bachelor diploma in material science \& engineering from National Cheng Kung University, Taiwan in 2009. During his bachelor study, he did a bachelor thesis on renewable hydrogen storage using metal alloy. He later worked in the leading material company in Taiwan as a quality control engineer for one year before he was admitted to the master programme of environmental science, Wageningen University (WUR). He finished his MSc with a major in environmental technology and a minor in environmental systems analysis. He continued his $\mathrm{PhD}$ in WUR, working on a dual-disciplinary research project combining environmental technology and systems analysis. The project focused on the conversion of mixed organic waste to valuable chemicals using microbial fermentation. During his $\mathrm{PhD}$, he received two prestigious awards from a Taiwanese industrial foundation and the Taiwanese government.

Wei-Shan Chen has started working as a postdoctoral researcher in WUR and Amsterdam institute for Advanced Metropolitan Solutions (AMS) since winter 2016. His postdoctoral work focuses on innovating the urban infrastructure for the application of resourcerecovering waste(water) technologies, which will ultimately promote the circular metabolism of the urban environment. 


\section{SENSE}

Netherlands Research School for the

Socio-Economic and Natural Sciences of the Environment

\section{I P L O M A}

For specialised PhD training

The Netherlands Research School for the Socio-Economic and Natural Sciences of the Environment (SENSE) declares that

\section{Wei-Shan Chen}

born on 29 June 1987 in Taipei City, Taiwan

has successfully fulfilled all requirements of the Educational Programme of SENSE.

Wageningen, 21 June 2017

the Chairman of the SENSE board

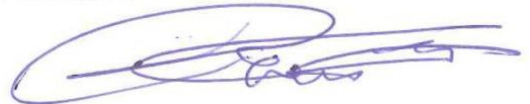

Prof. dr. Huub Rijnaarts the SENSE Director of Education

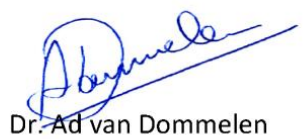

The SENSE Research School has been accredited by the Royal Netherlands Academy of Arts and Sciences (KNAW)

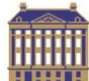

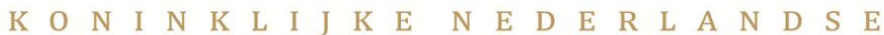

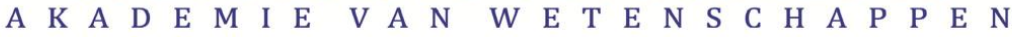




\section{(sesse)}

The SENSE Research School declares that Mr Wei-Shan Chen has successfully fulfilled all requirements of the Educational PhD Programme of SENSE with a work load of $44 \mathrm{EC}$, including the following activities:

\section{SENSE PhD Courses}

- Environmental research in context (2013)

- Research in context activity: 'Co-organizing the CHI-NED 4D Dialogue Seminar and a PhD study trip to China' (2016)

\section{Other PhD and Advanced MSc Courses}

- Imaging science: video and audio content in scientific communication, Wageningen University (2015)

- OLI stream analyzer workshop, Wageningen University (2013)

- Material flow analysis workshop, Technical University of Wien (2013)

- Life cycle assessment modelling of solid waste streams - application of the EASEWASTE model, Technical University of Denmark (2013)

- Microbial physiology, Wageningen University (2013)

\section{Management and Didactic Skills Training}

- Supervising three MSc students with thesis entitled 'Feasibility Study of Methanol-based Chain Elongation in Mixed culture' (2014), 'Optimization of Methanol-based Mixed Culture Chain Elongation' (2015) and 'Explore the feasibility of hydrogen as alternative electron donor for mixed-culture chain elongation' (2015)

- Supervising practical of the BSc course 'Biorefinery' (2013 and 2014)

\section{Oral Presentations}

- Caproate and butyrate formation in Mixed Culture Chain Elongation using methanol and acetate. $14^{\text {th }}$ World Congress on Anaerobic Digestion (AD14), 15-18 November 2015, Viña del Mar, Chile

- Environmental Impact Assessment (EIA) of Caproic Acid Production from Organic Waste. EXPO 2015 conference, LCA for "Feeding the planet and energy for life", 6-8 October 2015, Stresa, Italy

- Methanol as an alternative electron donor in Mixed Culture Chain Elongation. Environmental Technology for Impact conference (ETEI2015), 29-30 April 2015, Wageningen, The Netherlands

- Biofuel \& biochemical from organic waste. Masterclass: Biofuels - the past or the future?, 20 March 2014, Wageningen, The Netherlands

\section{SENSE Coordinator PhD Education}

Dr. ing. Monique Gulickx 


\section{Funding}

This research was funded by the Wageningen IPOP Research Programme Biorefinery, Wageningen Institute for Environmental and Climate Research (WIMEK) and ChainCraft B.V. The author would like to acknowledge their financial supports. 
